

Escola Superior de Tecnologia i Ciències Experimentals

Departament de Ciències Agràries i del Medi Natural

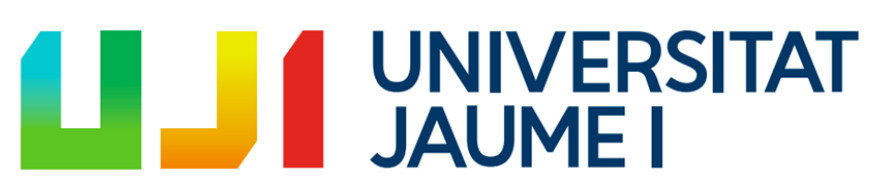

"Desarrollo de métodos de fenotipado de aroma en sandía y melón y su aplicación a la evaluación de líneas de mejora de portainjertos”

\section{Tesis Doctoral}

Presentada por:

Alejandro David Fredes Sivoplás

Dirigida por:

Dr. Salvador A. Roselló Ripollés

Dra. Mercedes Valcárcel Germes 



\section{LOS DIRECTORES DE TESIS:}

Dr. Salvador A. Roselló Ripollés, Catedrático de Universidad del Área de Producción Vegetal del Departamento de Ciencias Agrarias y del Medio Natural de la Universitat Jaume I de Castellón.

Dra. Mercedes Valcárcel Germes, Profesora Asociada del Área de conocimiento de Producción vegetal del Departamento de Ciencias Agrarias y del Medio Natural de la Universitat Jaume I de Castellón.

\section{HACEN CONSTAR QUE:}

Alejandro David Fredes Sivoplás, Químico por la Universidad de la República (Uruguay) y Máster en Técnicas Cromatográficas Aplicadas por la Universitat Jaume I de Castellón, ha realizado bajo su dirección el trabajo que, titulado "Desarrollo de métodos de fenotipado de aroma en sandía y melón y su aplicación a la evaluación de líneas de mejora de portainjertos” reúne las condiciones necesarias para optar al grado de Doctor.

Dr. Salvador A. Roselló Ripollés

Dra. Mercedes Valcárcel Germes

Castellón de la Plana, 27 de Julio 2017 



\section{"In God we trust, all others bring data"}

William Edwards Deming (1900-1993)

A Dios,

Mi Familia

y Amigos 

Gracias a mi Buen Padre por ayudarme hasta aquí.

En el plano personal, primero que nada, quiero dar las gracias a mis tutores de tesis, Mercedes "Merche" Valcárcel y Salvador "Salva" Roselló que con tanto esfuerzo y dedicación ha hecho posible esta tesis. Al inicio tomando la hazaña de tener un uruguayo en el laboratorio y luego a pesar de hablar el mismo idioma, han logrado entender mi cultura y llegar a consensos para enseñarme a investigar y desarrollar lo que hoy es una realidad. Sé que a veces no fui fácil, ya que "se me saltaron los Sivoplás" (como dice mi mamá), pero hemos llegado y les doy mi enhorabuena como dicen aquí, a ustedes en primer lugar por la paciencia y sobre todo porque he visto en este grupo comprensión, honestidad e integridad, los cuales en estos tiempos que corren son valores cada día menos frecuentes.

También agradezco a España, específicamente a la Generalitat Valenciana que por medio de la beca Santiago Grisolía, en primera instancia me permitió realizar el Master y parte del doctorado, que finalicé gracias a la ayuda de la beca Santander Universidades. Gracias por darme la oportunidad de venir a Castellón, conocer esta tierra, su cultura, su gente, sus comidas, poder investigar en ella, aprender de todas las situaciones vividas y sobre todo formarme como persona y profesional.

Muchas gracias a mis dos familias, la de sangre y la espiritual:

Gracias Mamá y abuela "Uya", porque si no fuera por ustedes dos no estaría donde estoy, si no fuera por esas tantas veces que me dijeron "no", "paciencia", "tu puedes", "no te rindas", "te amamos" y tantas frases que me vienen a la mente y podría llenar esta hoja, no hubiera terminado esta etapa ni otras. Gracias Papá por estar a mi lado y apoyarme. Gracias a mis hermanos: Gabi, Diego y Gus que a su manera han estado presentes desde lejos; y uno postizo que Dios me ha dado, que tanto me acompañó en este tiempo, dándome ánimo y sosteniéndome en momentos complicados, muchas gracias hermano-amigo Davide, así como a tu familia y tus amigos que tan bien me han tratado.

Gracias a mi familia de Uruguay, tíos/as, primos/as y toda la parentela por apoyarme. En especial a la tía Gloria por darme su pasión por la Química y a Pishta por el esfuerzo de venir a visitarme. 
Gracias a mis AMIGOS de Uruguay y España por siempre estar en diferentes momentos de mi vida. Gracias a mis compañeros del Master de la UJI por compartir parte de este trabajo conmigo. A ti Iulia y tu familia por compartir parte de sus vidas conmigo. Gracias a los amigos de la barra de Facultad de Química, por ayudarme incondicionalmente como hermanos en todo, aún en la distancia. Gracias a mis amigos uruguayos de Torredembarra por siempre recibirme con tanto cariño.

Por otro lado, gracias a las familias de "Reducto" en Montevideo y "Shalom" en Young, por cuidarme y ayudarme en los primeros pasos de universidad, por todos los amigos que quedaron en Uruguay pero que siempre estuvieron conmigo. Muchas Gracias a "Vida Nueva" por ser mi familia en Castellón, que decir de todos los amigos y hermanos que tengo allí, solo GRACIAS A TODOS por abrirme las puertas de sus casas, por alegrarse conmigo, llorar conmigo, compartir ricas comidas y tanto que no me cabe aquí.

Y que decir para los que estuvieron más cerca aún en estos últimos años?... en cada comida, en cada cena, en cada "almuerzo" o "merienda Internacional de Jueves"...

Gracias Raúl y Merche por darme los primeros aventones cuando era un recién llegado, y a pesar de estar en Valencia fueron de mucha ayuda.

Gracias Ginés, que sin ti las tardes y trabajos en el labo hubieran sido interminables, así como las ayudas en correcciones, búsquedas, detalles y muchas más cosas que has brindado en mi camino, y que decir de tus provisiones!.

Gracias María y Carlos, Sarita y Roberto, Tico y Aza, Marta y Hugo y Jorge por darme a lo largo de estos años de todo y más! Chicos las cosas que hemos compartido han sido increíbles y si no fuera por ustedes no hubiera podido terminar. Gracias por los viajes, los hospedaje en muchas ocasiones, los consejos, el ánimo y tanto... Gracias en serio por todo! no me dan las palabras.

Matías y Joana, mi ejemplo a seguir en muchas cosas, GRACIAS gurises, porque fueron los que enviaron aquel correo diciendo que había una oportunidad en España, me dieron pa' adelante siempre.

Gracias a todos los que han llegado hace poco o pasaron por el labo tanto de España como de Chile, Argentina, Portugal y Túnez, aportando siempre buenas experiencias. 
Gracias a los compañeros de los grupos del departamento de Ciencias Agrarias y del Medio Natural, por las ayudas y buena onda que han puesto siempre.

Gracias al grupo del COMAV de la Universidad Politécnica de Valencia, Jaime, Ana, Belén, Carmina y otros que de alguna u otra manera me han dado una gran mano en este trabajo.

Gracias a la gente del IUPA! Gracias Carlos, Ana María, Mercedes, Ariadna, Neus, Emma por todo el tiempo juntos; gracias a los profes, Félix, Ximo, Juanvi, Elena, María, Toni, Roque y otros que tanto aportaron a mi formación siendo referentes en la Química. Gracias a todos los que han ido pasando de diferentes países de los cuáles guardo tantos recuerdos si bien no los pondré a todos pero saben que ustedes también están.

¡ Muchas Gracias a todos; Moltes Gràcies a tots; Thank you very much! ¡ESTO HA SIDO POSIBLE POR CADA UNO DE USTEDES! 



\section{Índice General}

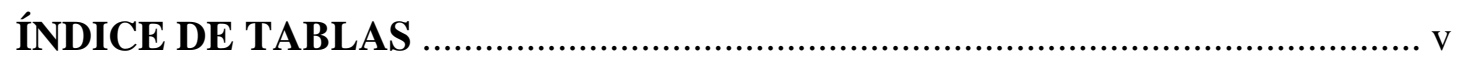

ÍNDICE DE FIGURAS …....................................................................... vii

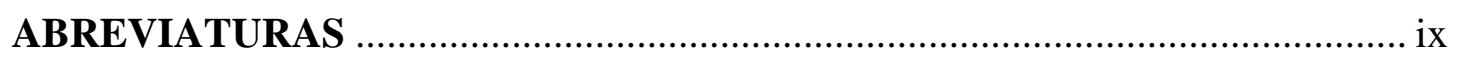

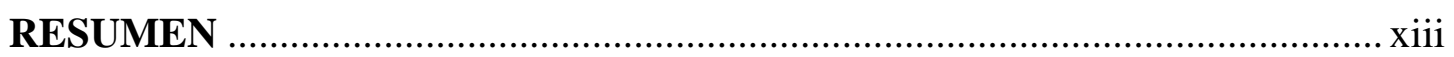

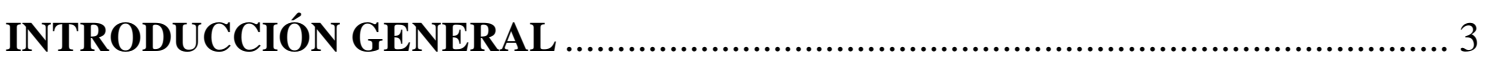

1. EL CULTIVO DE MELÓN Y SANDÍA …..................................................... 3

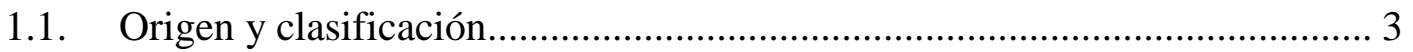

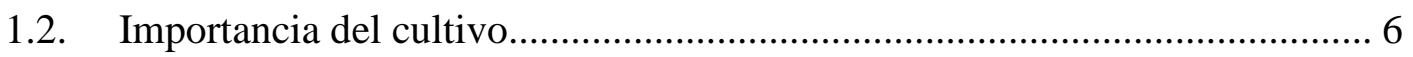

1.3. Tendencias productivas y de mercado ...................................................... 9

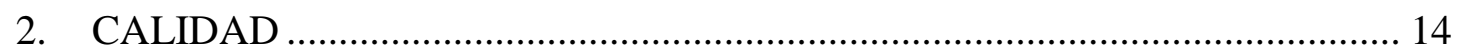

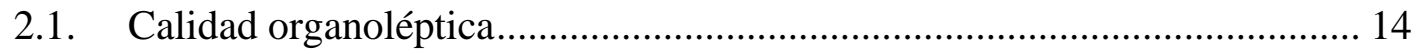

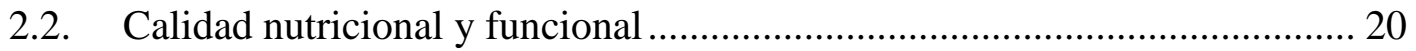

3. EVALUACIONES DE LAS CARACTERÍSTICAS DE CALIDAD

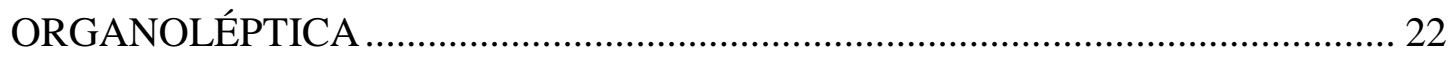

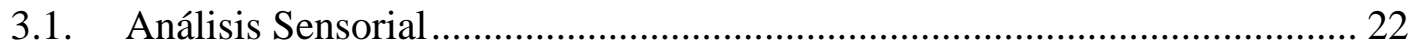

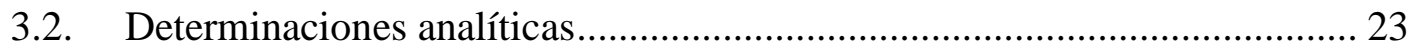

3.2.1. Determinación de ácidos y azúcares ................................................... 24

3.2.2. Determinación y cuantificación de compuestos orgánicos volátiles .... 25

3.2.2.1. Extracción y concentración......................................................... 25

3.2.2.2. Separación, identificación y cuantificación ................................... 29

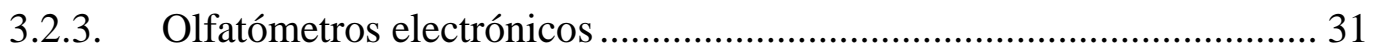

3.2.3.1. Sistema de toma de muestra...................................................... 35

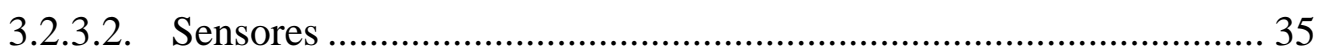

3.2.3.3. Métodos Quimiométricos ............................................................ 38

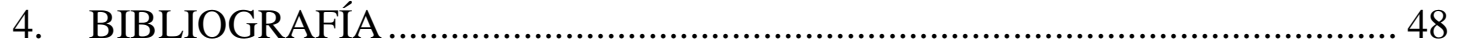

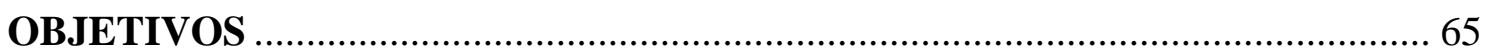

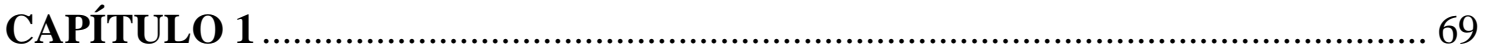

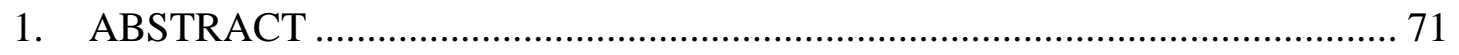

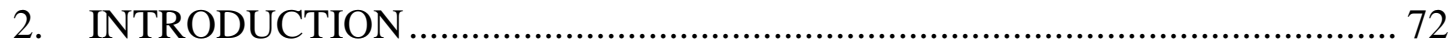

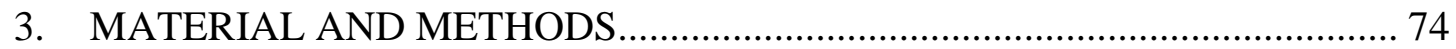




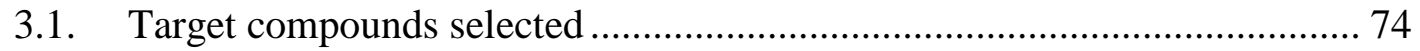

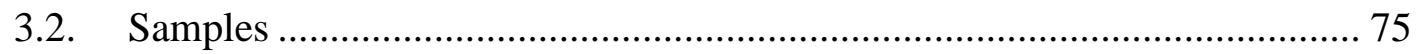

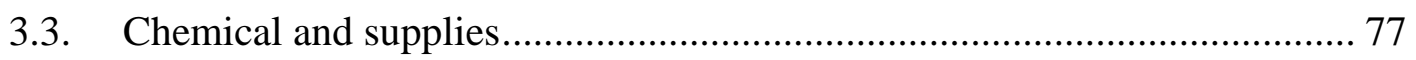

3.4. Optimised extraction procedures........................................................ 77

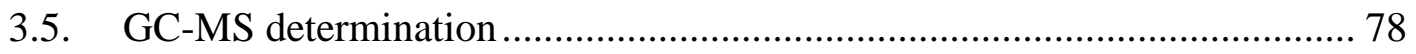

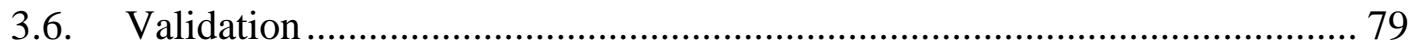

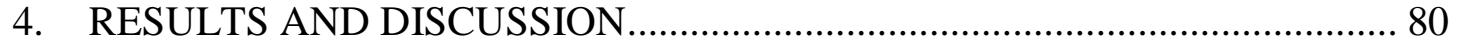

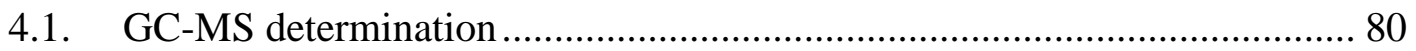

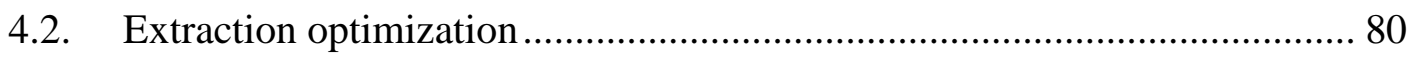

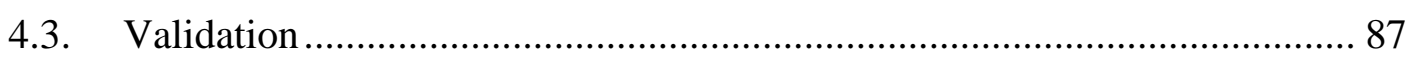

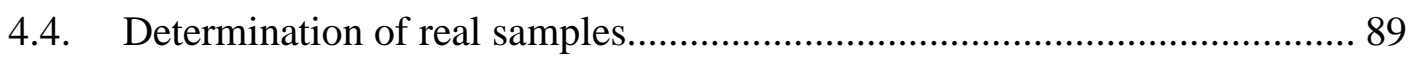

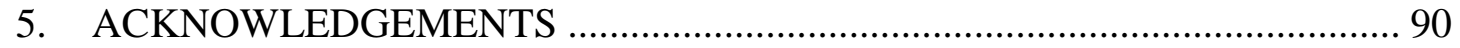

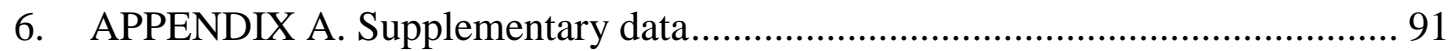

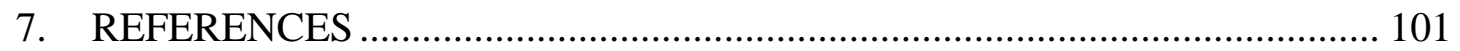

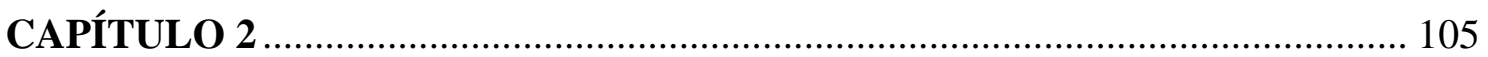

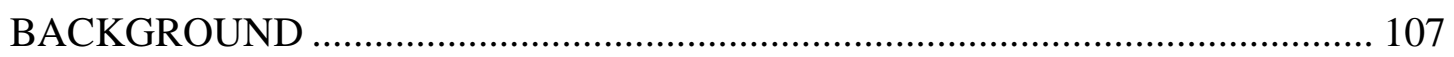

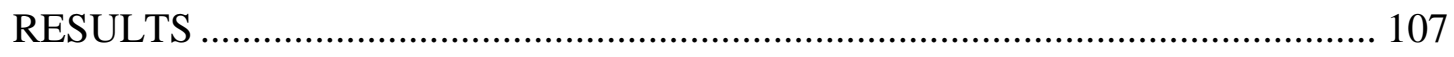

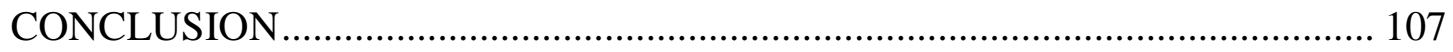

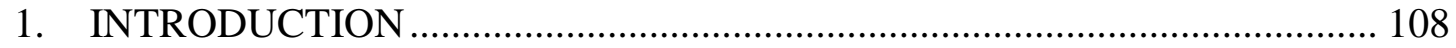

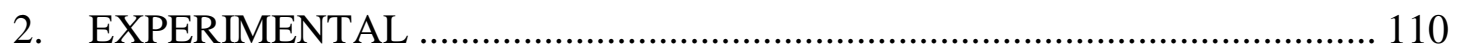

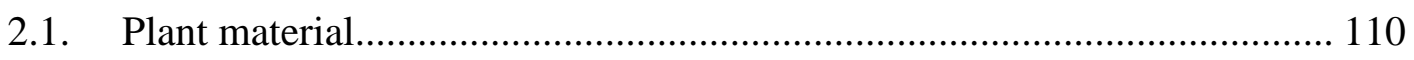

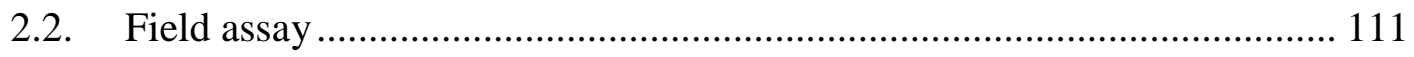

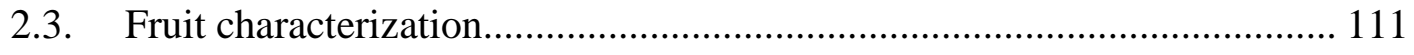

2.4. Reagents for metabolite analysis ....................................................... 112

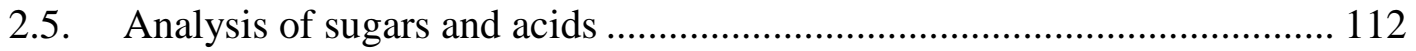

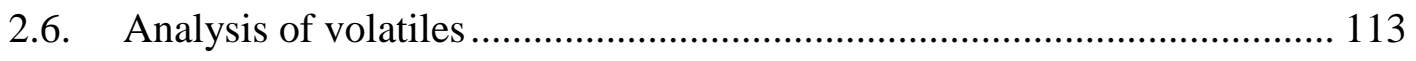

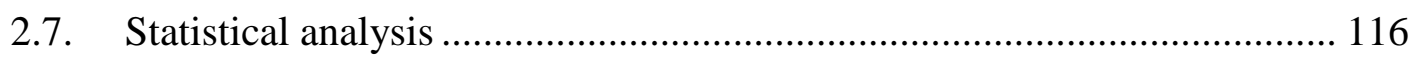

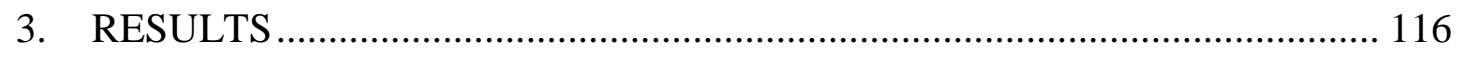

3.1. Effect of grafting on fruit morphology and flesh properties ..................... 116

3.2. Effect of grafting on flesh sugar and acid content ................................ 118

3.3. Effect of grafting on flesh aroma profile................................................ 119

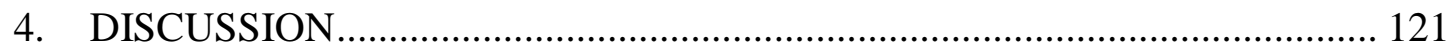

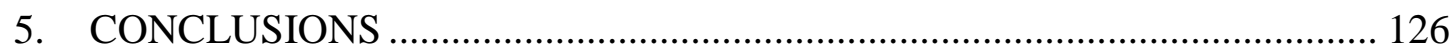




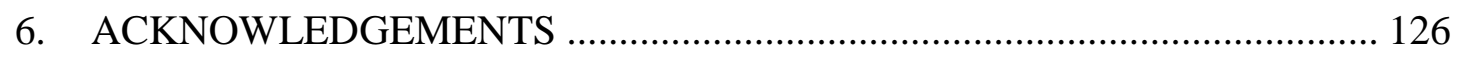

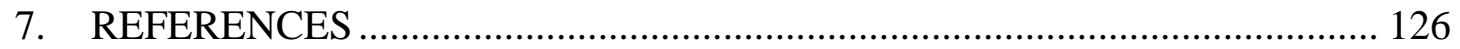

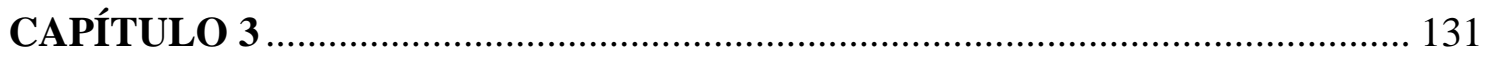

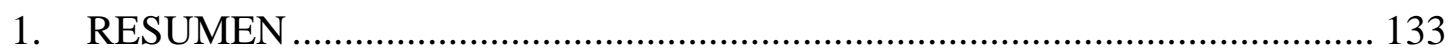

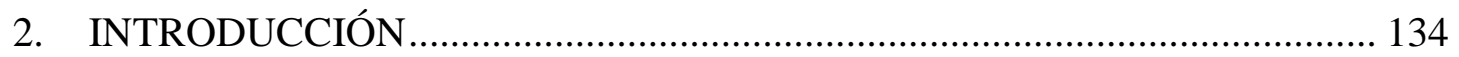

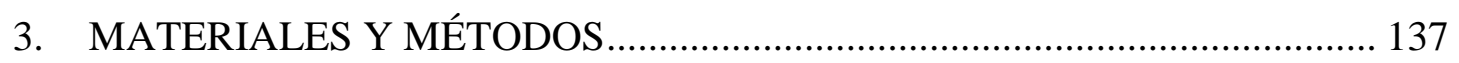

3.1. Diseño del ensayo y condiciones de cultivo............................................. 137

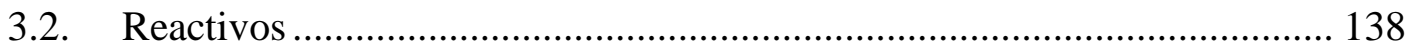

3.3. Determinación de compuestos volátiles por GC-MS .............................. 140

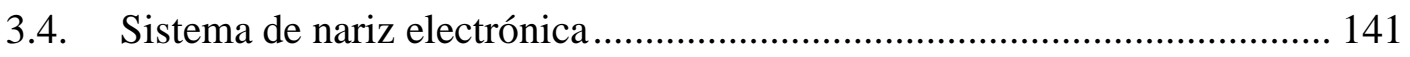

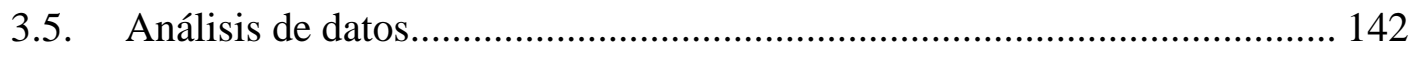

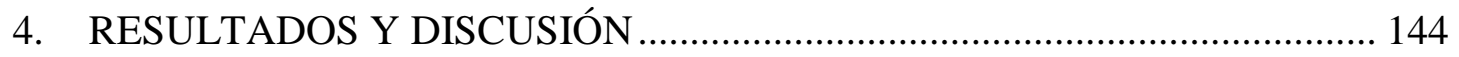

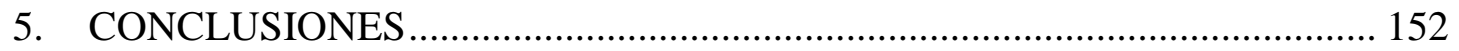

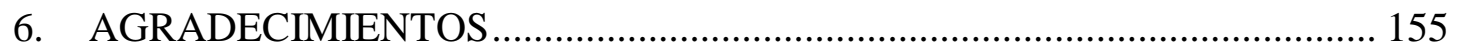

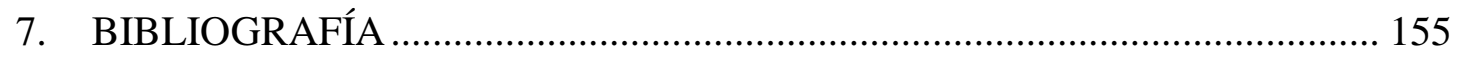

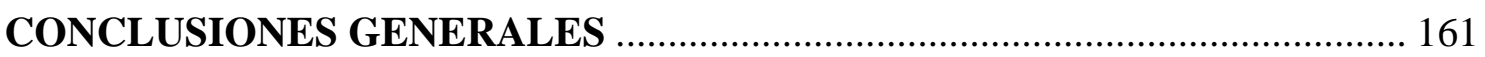

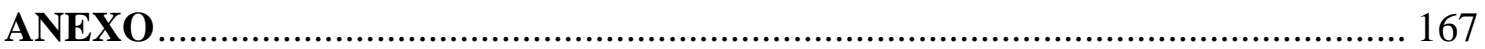





\section{INTRODUCCIÓN GENERAL}

Tabla 1. Evolución de la producción de melón de los principales países en millones de toneladas.

Tabla 2. Evolución de la producción de sandías de los principales países en millones de toneladas.

Tabla 3. Exportaciones de melón y sandía (en miles de toneladas) de las principales Comunidades Autónomas de España.

Tabla 4. Principales compuestos orgánicos aromáticos presentes en muestras de melón y sandía.

Tabla 5. Composición de melón y sandía en 100 g de porción comestible.

Tabla 6. Características de diferentes olfatómetros electrónicos comerciales.

\section{CAPÍTULO 1}

Table 1. List of studied target compounds. Chromatographic parameters (retention time (Rt), retention index (RI) and quantitation ion (Quan Ion)) obtained from GC-MS chromatograms. Validation parameters obtained after application of optimized procedure (P\&T extraction using EnviCarb $500 \mathrm{mg}$ cartridges, $30 \mathrm{~g}$ of melon sample).

Table 2. Results obtained in the optimization of volatiles extraction in muskmelon samples using RSM. Significant model factors, parameters of model goodness of fit and confirmation results in the optimum extraction conditions predicted are indicated.

Table 3. Real sample determination.

Table S1. Review of the volatiles compounds presented in melon samples reported in eleven articles. The table shown the reported compounds, CAS numbers, references with the concentration (in the cases that they are quantitate) and comments about sensory descriptors or significant features. 


\section{CAPÍTULO 2}

Table 1. Volatiles analyzed in the watermelon samples.

Table 2. Characteristics of watermelon fruits (Citrullus lanatus F1 Oneida) harvested from non-grafted plants (NG), self-grafted plants (SG) and plants grafted the experimental Cucurbita (C. maxima $\times$ C. moschata) hybrid F1 (GMM1), the commercial Cucurbita hybrid Cobalt (GMM2) and one new experimental citron melon (Citrullus lanatus var. citroides) (GC).

Table 3. Sugars and acids concentrations of watermelon fruits (Citrullus lanatus cv. F1 Oneida) harvested from non-grafted plants (NG), self-grafted plants (SG) and plants grafted onto the experimental Cucurbita (C. maxima $\times$ C. moschata) hybrid F1 (GMM1), the commercial Cucurbita hybrid Cobalt (GMM2) and one new experimental citron melon (Citrullus lanatus var. citroides) (GC).

Table 4. Mean contents of the volatiles quantified in watermelon fruits (Citrullus lanatus cv. F1 Oneida) harvested from non-grafted plants (NG), self-grafted plants (SG) and plants grafted onto the experimental Cucurbita (C. maxima $\times$ C. moschata) hybrid F1 (GMM1), the commercial Cucurbita hybrid Cobalt (GMM2) and one new experimental citron melon (Citrullus lanatus var. citroides) (GC).

\section{CAPÍTULO 3}

Tabla 1. Concentraciones (ng $\left.\mathrm{mL}^{-1}\right)$ de los principales volátiles utilizados en el patrón sintético para corrección de deriva.

Tabla 2. Modelos de clasificación (calibrado y validaciones cruzadas) obtenidos por PLS-DA de las muestras analizadas por GC-MS (A) y E-Nose (B). Donde los valores de sensibilidad y especificidad van desde 0 (mínimo) a 1 (máximo).

Tabla 3. Modelos con buena predicción de contenidos de volátiles $\left(\mathrm{ng} \mathrm{g}^{-1}\right)$ a partir de respuestas de los sensores de nariz electrónica.

Tabla 4. Modelos de predicción aproximada de contenidos de volátiles ( $\left.\mathrm{ng} \mathrm{g}^{-1}\right)$ a partir de respuestas de los sensores de nariz electrónica. 


\section{INTRODUCCIÓN GENERAL}

Figura 1. Diversidad de melones y sandías.

Figura 2. Producción de melón y sandía en secano y regadío en el año 2014 (\% toneladas).

Figura 3. Producción de melones en miles de toneladas de las comunidades más relevantes de España en el año 2014.

Figura 4. Producción de sandías en miles de toneladas de las comunidades más relevantes de España en el año 2014.

Figura 5. Evolución del porcentaje de sandía injertada frente a la sin injertar en semilleros de la Comunidad Valenciana.

Figura 6. Ejemplo de injerto.

Figura 7. Oferta de frutos de melón y sandía cortados en punto de venta.

Figura 8. Esquema comparativo del funcionamiento de la nariz humana versus la "nariz electrónica".

Figura 9. Resumen de clasificación de los principales métodos estadísticos utilizados en nariz electrónica.

\section{CAPÍTULO 1}

Figure 1. a) Total ion chromatogram (TIC) of a standard mixture of 61 volatile compounds and b) TIC of a Charentais muskmelon sample extract. See Table 1 for peak identification.

Figure 2. Response surface plots showing the effect of A: sample weight $(\mathrm{g}), \mathbf{B}$ : extraction time (min) and $\mathbf{C}$ : gas flow (\% max of the scale, $\left.1.6 \mathrm{~L} \mathrm{~min}^{-1}\right)$ on the peak area of prominent volatile compounds found in muskmelon and watermelon. The selected conditions with the weighted desirability function to maximize global extraction are represented with a vertical black solid line. 


\section{CAPÍTULO 2}

Figure 1. MANOVA biplot of fruit volatiles. Circles represent Bonferroni confidence intervals for the mean value of each material. In all cases the scion corresponds to $\mathrm{cv}$. 'Oneida RZ'. NG: 'Oneida RZ' non-grafted; SG: self-grafted; GC: grafted on citron melon; GMM1: grafted on experimental Cucurbita F1 hybrid; GMM2: grafted on 'Cobalt RZ' a commercial Cucurbita F1 hybrid.

\section{CAPÍTULO 3}

Figura 1. Perfil de volátiles (promedio \pm desviación estándar expresados en $\mathrm{ng} \mathrm{g}^{-1}$ ) para frutos de sandía (Citrullus lanatus F1 Oneida) sin injertar (NG), autoinjertados $(\boldsymbol{S G})$ e injertados en: patrón experimental de Cucurbita (C. maxima x C. moschata) híbrido F1 (GMM1), híbrido comercial F1 de Cucurbita (C. maxima x C. moschata) Cobalt (GMM2), patrón de Cucurbita (C. pepo) (GPepo) y patrón experimental de melón citroides (Citrullus lanatus var. citroides) $(\boldsymbol{G C})$.

Figura 2. Mapas de similitud obtenidos por PLS-DA de los datos de perfil volátil obtenidos por cromatografía (A) y nariz electrónica (B) a partir de frutos de sandía (Citrullus lanatus F1 Oneida) sin injertar $(\boldsymbol{N G})$, autoinjertados $(\boldsymbol{S G})$ e injertados en: patrón experimental de Cucurbita (C. maxima x C. moschata) híbrido F1 (GMM1), híbrido comercial F1 de Cucurbita (C. maxima x C. moschata) Cobalt (GMM2), patrón de Cucurbita (C. pepo) (GPepo) y patrón experimental de melón citroides (Citrullus lanatus var. citroides) $(\boldsymbol{G C})$. 
ADN

ANN

ART

ATT

CA

CE

CI

COEXPHAL

CP

CZE

DFA

DHS

EI

ELM

FAO

FEPEX

FID

Fuzzy ARTMAP

GA

GC
Ácido desoxirribonucleico.

(Artificial Neural Network) Redes Neuronales Artificiales.

(Adaptative Resonance Theory) Teoría de resonancia adaptativa.

(Alcohol Acyltransferase) Alcohol aciltransferasas.

(Clusters Analysis) Análisis de clúster.

Comunidad Europea.

(Chemical Ionization) Ionización química.

Asociación de Organizaciones de Productores de Frutas y

Hortalizas de Almería.

(Conductive Polymers) Polímeros conductores.

(Capillar Zone Electrophoresis) Electroforesis capilar zonal.

(Discriminat Function Analysis) Análisis de función discriminante.

(Dinamic Head Space) Espacio de cabeza dinámico.

(Electron Ionization) Ionización de impacto electrónico.

(Extreme Learning Machine) Máquinas de aprendizaje extremo.

Organización de las Naciones Unidas para la Alimentación y la Agricultura.

Federación Española de Asociaciones de Productores Exportadores de Frutas y Hortalizas.

(Flame Ionization Detector) Detector de ionización de llama.

(Fuzzy Adaptative Resonance Theory) Lógica de mapas ART difusa.

(Genetic Algorithms) Algorítmos genéticos.

(Gas Chromatography) Cromatografía de gases. 
GCO

HPLC

HS

HSSE

ICR

i-PLS

IT

LVQ

$\mathbf{m} / \mathbf{z}$

MAPAMA

MLP

MLR

MOS

MOSFET

MS

NN

P\&T

PARC

PCA

PCR
(Gas Chromatography-Olfactometry) Cromatógrafía de gases Olfatometría.

(High Performance Liquid Chromatography) Cromatografía líquida de alta resolución.

(Head-Space) Espacio de cabeza.

(Head Space Sorptive Extraction) Extracción por adsorción en espacio de cabeza.

(Ion Cyclotron Resonance) Resonancia ion-ciclotrón.

(interval PLS) intervalo-PLS.

(Ion Trap) Trampa de iones.

(Learn Quantification Vector) Cuantificación del vector de aprendizaje.

Relación masa/carga.

Ministerio de Agricultura y Pesca, Alimentación y Medio Ambiente.

(Multi-Layer Perceptron) Perceptrón multicapa.

(Multiple Linear Regression) Regresión lineal múltiple.

(Metal Oxide Semiconductor) Sensores de óxidos metálicos semiconductores.

(Metal Oxide Semiconductor Field Effect Transistor) Sensores de óxidos metálicos semiconductores basados en efecto de campo.

(Mass Spectrometry) Espectrometría de masas.

(Nearest Neighbor) Análisis de vecino más próximo.

(Purge and Trap) Purga y Trampa.

(Pattern Recognition) Reconocimiento de patrones.

(Principal Component Analysis) Análisis de componentes principales.

(Principal Component Regression) Regresión del componente 
principal.

PLS

PLS-DA

ppb

ppm

ppt

PTV

Q

QCM

RAE

RBF

RMSECV

SAW

SBSE

SHS

SOM

Sp.

SPME

SPS

SS

Subsp.
(Parcial Least Square) Regresión parcial mínimo cuadrática.

(Parcial Least Square - Discriminant Analysis) Análisis discriminante basado en regresiones parciales mínimo cuadráticas.

Partes por billón.

Partes por millón.

Partes por trillón.

(Programmed Temperature Vaporizer) Vaporizador de temperatura programada.

(Quadrupole) Cuadrupolo.

(Quartz Crystal Microbalance) Sensores de microbalanzas de cristal de cuarzo.

Real Academia Española.

(Radial Basis Function) Redes de funciones de base radial.

(Root Mean Square Error of Cross-Validation) Error mínimo cuadrático de validación cruzada.

(Surface Acoustic Waves) Sensores de superficie de onda acústica.

(Stir Bar Sorptive Extraction) Extracción por adsorción en barra agitadora.

(Static Head Space) Espacio de cabeza estático.

(Self- Organizing Map) Mapas auto-organizados.

Especie.

(Solid Phase Microextraction) Microextracción en fase sólida.

(Sucrose Phosphate Syntase) Sacarosa Fosfato Sintasa.

(Sucrose Syntase) Sacarosa Sintasa.

Subespecie. 
(Support Vector Machine) Máquinas de vectores soporte.

TDU

(Thermal Desorption Unit) Unidad de desorción térmica.

TOF

(Time-Of-Flight) Tiempo de vuelo. 
El melón (Cucumis melo L.) y la sandía (Citrullus lanatus Thunb.) son frutos muy consumidos en épocas de calor ya que son alimentos refrescantes por su alto contenido en agua y su bajo aporte calórico.

Ambas especies tienen en común que es difícil intuir su calidad interna a partir del aspecto exterior. Debido a la dureza de ambos en estado maduro, ni siquiera es posible para un consumidor estándar apreciar el grado de maduración de la fruta. Por esta razón, el auge del consumo de ambos se ha conseguido a través de la oferta de fruta cortada y, en la actualidad, a través de productos de cuarta gama. En cualquier caso, la consolidación de su mercado pasa necesariamente por satisfacer las expectativas de calidad organoléptica demandadas por el consumidor.

Por esta razón, los programas de mejora que pretenden abarcar mercados de calidad tratan de desarrollar nuevas variedades que maximicen la calidad organoléptica. Si bien la mejor forma de determinar el sabor es a través de paneles de cata, su uso se hace inviable en programas de mejora. Por ello, es necesario contar con herramientas de análisis objetivas que permitan cuantificar los compuestos involucrados en la definición del sabor. En el caso de estas especies, la calidad vendría fundamentalmente determinada por el contenido en azúcares (glucosa, fructosa y sacarosa) y en compuestos volátiles relacionados con el aroma.

La presente tesis ha tratado de abordar este problema en el caso del aroma. En primer lugar, se abordó la optimización de un método de cuantificación basado en cromatografía de gases acoplada a espectrometría de masas (GC-MS) de los principales compuestos volátiles de ambas especies. La extracción de volátiles es quizá el paso más complejo del análisis y que puede presentar más variabilidad. Su optimización se consiguió mediante el uso de superficies de respuesta multivariante a partir del método de extracción por purga y trampa. Las condiciones óptimas de extracción se obtuvieron empleando $30 \mathrm{~g}$ de muestra, 49 min de tiempo de extracción y un flujo de gas portador de $1.6 \mathrm{~L} \mathrm{~min}^{-1}$. Este método fue validado e hizo posible la extracción de varias muestras simultáneamente. El menor coste y rapidez de extracción lo hace ideal para analizar un número elevado de muestras. 
Este método fue aplicado con éxito a la evaluación del efecto del injerto sobre el perfil de volátiles de la sandía. Esta técnica está muy extendida en el manejo de esta especie y sin embargo su efecto sobre el aroma ha sido muy poco estudiado. Así, se comparó la composición de volátiles de los frutos de sandía (F1 Oneida) injertada en portainjertos de híbridos de Cucurbita, uno experimental (F1 C. maxima x C. moschata) y uno comercial (F1 Cobalt), y un portainjerto experimental de melón citroides (Citrullus lanatus var. citroides) respecto a los de sandía autoinjertada y sin injertar. Los resultados demostraron que existe un efecto del injerto sobre el perfil de compuestos volátiles, lo que resalta la importancia de valorar este aspecto en programas de mejora de líneas portainjerto. Por otro lado, en el estudio se demostró que la variedad citroides puede representar una alternativa interesante a los híbridos de Cucurbita generalmente usados, ya que presentó un perfil de volátiles similar a las plantas sin injertar. Además, se comprobó que los frutos provenientes de portainjertos híbridos de Cucurbita presentaron una mayor acumulación de Z-6-nonen-1-ol, que está relacionado al aroma de calabaza y que podría disminuir la calidad del aroma de sandía.

A pesar de la eficacia del método obtenido, la cromatografía de gases sigue requiriendo equipos caros, personal cualificado, largos tiempos de análisis y un importante gasto en fungible. Por tanto, si bien es ideal para contrastar la idoneidad de un número limitado de líneas en fases finales de un programa de mejora, su uso no sería viable a lo largo del mismo.

En este contexto, se abordó la utilización de un olfatómetro electrónico con 18 sensores de metales semiconductores como un sistema alternativo de fenotipado del aroma en sandía, que permitiera evaluar grandes cantidades de material en tiempos de análisis contenidos y con un gasto mínimo en fungible.

El primer paso consistió en desarrollar un patrón sintético del aroma que permitiera corregir la variación intra- e inter-día, para a continuación aplicarlo a la evaluación de líneas de mejora de portainjertos en sandía.

Mediante esta técnica, se pudo desarrollar mapas de similitud en el perfil de volátiles que permiten seleccionar los materiales más parecidos a la sandía sin injertar. Además estos mapas mostraron una completa sintonía con la misma información obtenida mediante GC-MS. Adicionalmente, utilizando regresiones i-PLS, se obtuvieron modelos matemáticos semicuantitativos que permitieron obtener valores de concentración 
aproximada para algunos compuestos importantes como el Z-6-nonen-1-ol, ya señalado por su efecto negativo en portainjertos de Cucurbita. De esta forma, el uso de olfatómetro electrónico permitirá en fases iniciales de programas de mejora seleccionar aquellas líneas que afectan menos al perfil volátil y que presentan menor acumulación de compuestos considerados como negativos. 



\section{INTRODUCCIÓN GENERAL}

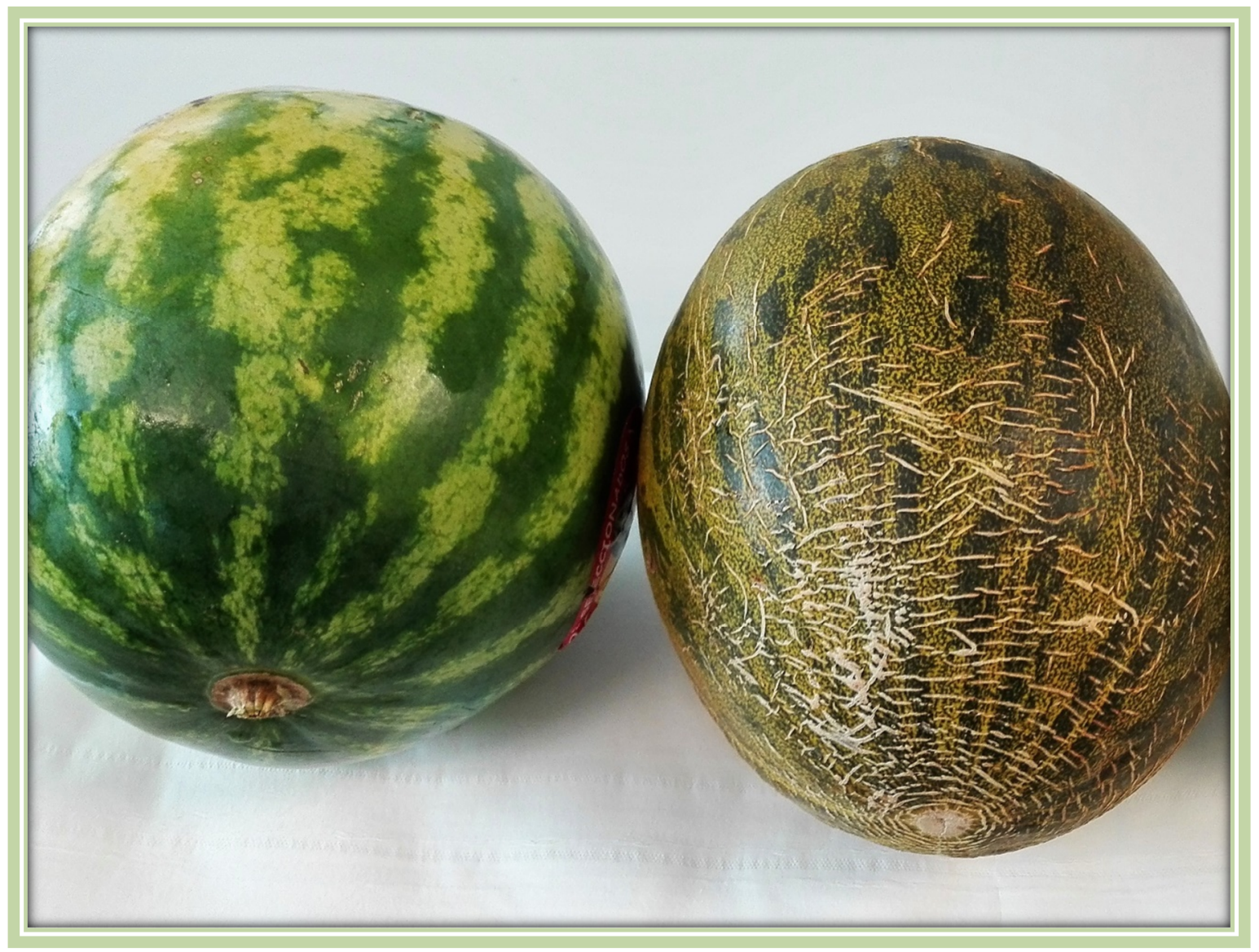





\section{EL CULTIVO DE MELÓN Y SANDÍA}

\subsection{Origen y clasificación}

El melón (Cucumis melo L.) y la sandía (Citrullus lanatus Thunb.) pertenecen a la familia de las cucurbitáceas, que además incluye otras especies como el pepino (Cucumis sativus L.), las calabazas [Cucurbita pepo L., Cucurbita maxima Duch. ex Lam., Cucurbita moschata (Duch. ex Lam.) Duch. ex Poir., Cucurbita argyrosperma Huber, Cucurbita ficifolia Bouché y Lagenaria siceraria (Mol.) Standl.] y el calabacín (Cucurbita pepo L.; subsp. pepo).

Tradicionalmente el origen del melón se ha adjudicado a África (Monforte \& Alvarez, 2006). No obstante, los últimos análisis realizados apuntan a un origen en Asia o Australia, llegando el melón a África más tarde (Monforte et al., 2014). En cualquier caso, sí que está claro que los melones silvestres apenas llegan a los $50 \mathrm{~g}$ de peso y probablemente se empezaron a aprovechar por sus semillas (Pitrat et al., 2000, 1999). Tras el inicio de la domesticación, el aprovechamiento de la pulpa daría lugar a selecciones que conllevaron el aumento de tamaño del mesocarpo (Monforte et al., 2014).

La sandía también tiene un origen africano (Pitrat et al., 1999). Aunque las primeras hipótesis hablan de un origen sudafricano, estudios más recientes han situado su posible origen al oeste de África cerca del desierto del Kalahari, Namibia (Chomicki \& Renner, 2015). Las sandías silvestres son pequeñas y redondas y su sabor es muy amargo. Durante la domesticación se realizó una profunda presión de selección especialmente sobre el cromosoma 3 que alberga genes relacionados con la maduración, la señalización mediada por azúcares, el transporte de los carbohidratos y el desarrollo del fruto (Reddy et al., 2014).

Las semillas más antiguas de sandía tienen más de 5000 años y se han localizado en Libia (Wasylikowa \& Van Der Veen, 2004), mientras que los registros históricos parten milenios más tarde en Egipto, con representaciones en tumbas como la de Tutankamón.

Ambas especies presentan gran diversidad (Figura 1), por lo que su clasificación es relativamente compleja comparada con otros cultivos. Según Monforte \& Alvarez (2006) el género Cucumis se divide en dos subgéneros: Melo representado por el melón, el kiwano 
(C. metuliferus Mey. ex Naud.), el pepinillo de Indias (C. Anguria L.) y treinta especies silvestres africanas; y el subgénero Cucumis, donde encontramos el pepino (C. sativus L.) y su especie silvestre.

Dentro de la especie C. melo aparecen dos subespecies (Pitrat et al., 2000):

- Subespecie agrestis donde podemos encontrar frutos que van desde no climatéricos, alargados, de piel lisa, carne firme blanca y no dulce (var. conomon) consumidos en ensaladas o conservas como el pepino; pasando por frutos climatéricos redondos o achatados, piel lisa, carne dulce y poco aromática como los de var. makuwa; hasta frutos con forma de pera, sin aroma y poco dulces (var. chinesis). Dentro de esta subespecie también hay dos variedades con frutos no aromáticos y no dulces que suelen consumirse preferentemente cocidos: var. momordica con frutos climatéricos, alargados o achatados de piel lisa que se cosechan antes de la madurez para evitar que estallen; y var. acidulus que presenta frutos ovales o elípticos, de piel lisa color naranja, carne blanca, firme y crujiente.

- Subespecie melo

○ var. cantalupensis. Frutos de tamaño medio, de forma achatados a ligeramente ovales, acostillados y lisos, de carne dulce, color salmón, aromáticos y climatéricos. Ejemplos: Cantaloups o Cantalupos, Charentais, Ogen, Vendratais.

○ var. reticulatus. Frutos achatados a ligeramente ovales, de piel muy reticulada, con o sin costillas, carne dulce y de color salmón. Aromáticos y climatéricos. Ejemplos: Galia, Villaconejos, Verde oloroso reticulado, Grade alargado.

○ var. inodorus. Frutos que van de redondeados a elípticos, de color que varía del blanco al verde oscuro uniforme o con manchas, con frecuencia la piel es rugosa con o sin costillas, la carne blanca y jugosa, sin aroma y no climatéricos. Ejemplos: Piel de sapo, Rochet, Tendral, Amarillo, Valenciano amarillo, Honeydew.

○ var. flexuolus. Frutos muy largos (más de 6 veces más largos que anchos) de piel verde claro, con bandas verde claro/ verde oscuro, 
acostillados o rugosos. El fruto maduro no es dulce, la carne es blanca y son climatéricos. Ejemplos: Alficoz.

○ Otros: var. adana, var. chandalak, var. ameri, var. chate, var. tibish, var. dudaim y var. chito.

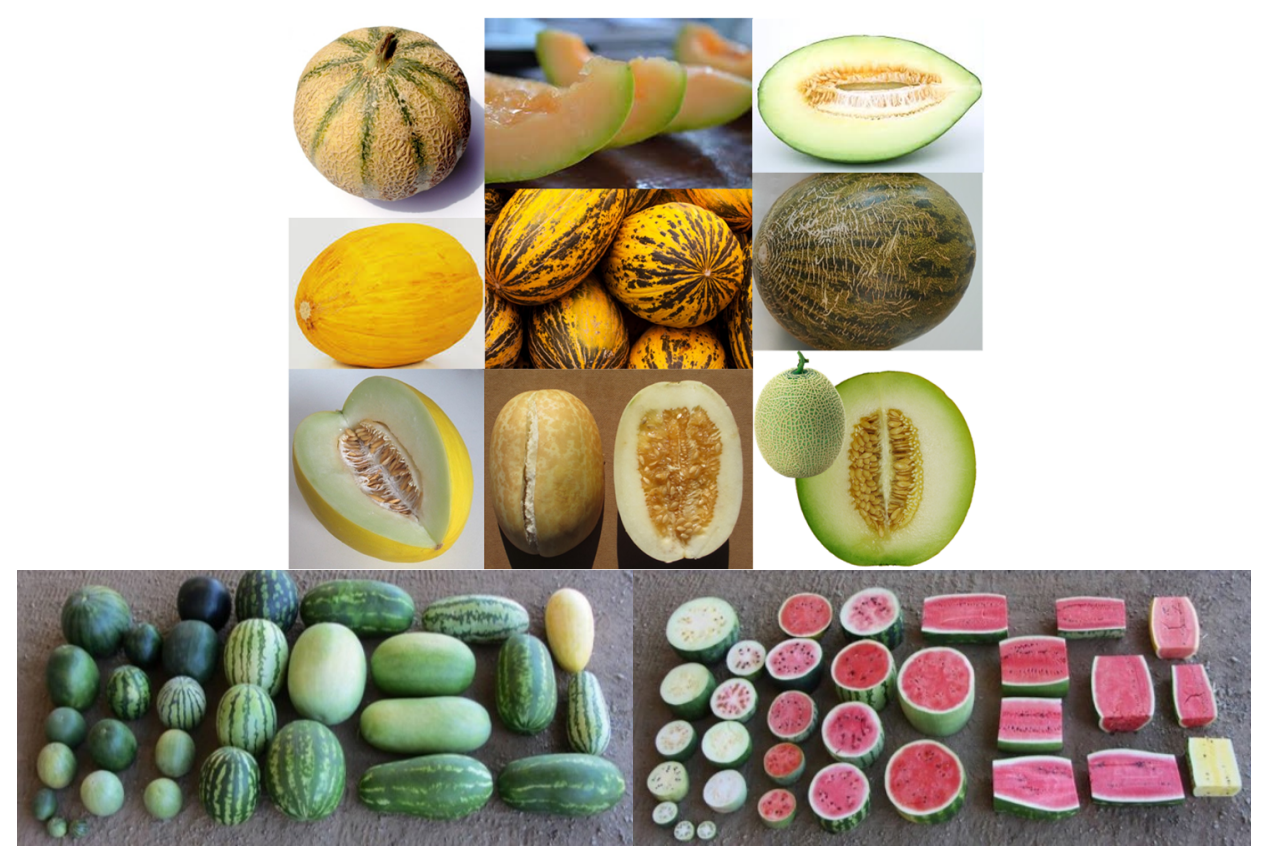

Figura 1. Diversidad de melones y sandías (Cortesía The Davis Enterprise, 2014)

En el caso de la sandía, la especie $C$. lanatus se subdivide en dos subespecies: $C$. lanatus var. lanatus, que incluye la sandía dulce cultivada y la $C$. lanatus var. citroides, que incluye materiales amargos utilizados en conserva.

Dentro de la especie cultivada no hay variedades botánicas reconocidas, aunque suelen ser comunes las clasificaciones en función de la forma, la coloración y la presencia de semillas (Maroto, 2002). Este último carácter es especialmente importante en la actualidad, ya que el aumento del cultivo de esta especie se ha ligado entre otros aspectos al desarrollo de variedades triploides que obvian el problema de la presencia de semillas durante el masticado de la fruta. Así los materiales cultivados pueden clasificarse como:

- De frutos redondeados con semillas y pulpa roja:

- Corteza oscura: Sugar Baby, Catalana Precoz, Pata Negra, Valenciana común de semilla negra, Pileña, Sayonara.

- Corteza rayada: Crimson Sweet, Imperial. 
- De frutos alargados con semillas y pulpa roja:

- Corteza oscura: Oden, Blacklee.

- Corteza rayada: Klondike, Príncipe Charles, Fairfax, Congo.

- De frutos redondeados y pulpa amarilla: Yellow doll, Sorpresa, Graciosa, Angela.

- Variedades sin semillas:

- Corteza oscura: Sin, Fummy, Pasión, Fashion.

- Corteza rayada: Reina de Corazones, Jack of Hearts, Duquesa.

\subsection{Importancia del cultivo}

Aunque el melón y la sandía son frutos consumidos principalmente en fresco, también son utilizados en la elaboración de confitados, zumos y "sorbetes" (Baixauli, 2002; Reche, 2008). El consumo y auge del cultivo de ambas cucurbitáceas se ha visto limitado durante mucho tiempo por dos inconvenientes. El primero es que se trata de frutos muy grandes que no suelen consumirse en una única comida. El segundo es que el aspecto exterior no refleja el grado de maduración del fruto. Ambos, se han visto solventados por la comercialización de fruta partida. Así el consumidor adquiere menos cantidad de producto y además es capaz de apreciar el estado de la pulpa. Siguiendo a este éxito, en la actualidad está en auge la comercialización dentro del segmento de cuarta gama.

Estos cultivos tienen una gran importancia económica en España. Según los últimos datos de la Organización de las Naciones Unidas para la Alimentación y la Agricultura, en el año 2014, España ocupó el $7^{\circ}$ lugar a nivel mundial en la producción de melón (Tabla 1) y $11^{\circ}$ en la producción de sandía (Tabla 2). Además, es el país con el mayor volumen de producción en Europa, exportando a la comunidad Europea más del 50\% de su producción de sandías (Pérez, 2015).

En España, la producción de melón en la última década ha experimentado un cierto descenso (Tabla 1), mientras que la producción de sandías ha permanecido aproximadamente constante (Tabla 2). La Asociación de Organizaciones de Productores de Frutas y Hortalizas de Almería (COEXPHAL) atribuye el descenso experimentado por el cultivo del melón a la competencia en el comercio europeo de zonas con menores costes de 
producción como Marruecos, Brasil o Costa Rica.

Tabla 1. Evolución de la producción de melón de los principales países en millones de toneladas (FAO, 2014). No incluye sandías.

\begin{tabular}{|l|c|c|c|c|c|c|c|c|c|c|c|}
\cline { 2 - 13 } & $\mathbf{2 0 0 4}$ & $\mathbf{2 0 0 5}$ & $\mathbf{2 0 0 6}$ & $\mathbf{2 0 0 7}$ & $\mathbf{2 0 0 8}$ & $\mathbf{2 0 0 9}$ & $\mathbf{2 0 1 0}$ & $\mathbf{2 0 1 1}$ & $\mathbf{2 0 1 2}$ & $\mathbf{2 0 1 3}$ & $\mathbf{2 0 1 4}$ \\
\hline China & 12,08 & 13,05 & 13,92 & 14,21 & 16,07 & 12,22 & 17,25 & 17,26 & 13,39 & 14,41 & 14,83 \\
\hline Turquía & 1,75 & 1,83 & 1,77 & 1,66 & 1,75 & 1,68 & 1,61 & 1,65 & 1,71 & 1,70 & 1,71 \\
\hline Irán & 1,22 & 1,58 & 1,37 & 1,66 & 1,33 & 1,28 & 1,27 & 1,44 & 1,44 & 1,46 & 1,48 \\
\hline Egipto & 0,56 & 0,57 & 0,75 & 0,83 & 0,92 & 0,92 & 1,08 & 1,04 & 1,01 & 0,92 & 1,05 \\
\hline Kazajistán & 0,15 & 0,16 & 0,16 & 0,19 & 0,22 & 0,27 & 0,33 & 0,46 & 0,83 & 0,77 & 0,91 \\
\hline EE.UU. & 1,23 & 1,18 & 1,13 & 1,11 & 1,04 & 1,04 & 1,04 & 1,02 & 0,91 & 0,99 & 0,79 \\
\hline España & $\mathbf{1 , 0 7}$ & $\mathbf{1 , 0 9}$ & $\mathbf{1 , 0 9}$ & $\mathbf{1 , 1 8}$ & $\mathbf{1 , 0 4}$ & $\mathbf{0 , 9 8}$ & $\mathbf{0 , 9 3}$ & $\mathbf{0 , 8 7}$ & $\mathbf{0 , 8 8}$ & $\mathbf{0 , 8 6}$ & $\mathbf{0 , 7 5}$ \\
\hline Marruecos & 0,67 & 0,65 & 0,65 & 0,73 & 0,74 & 0,89 & 0,57 & 0,78 & 0,72 & 0,70 & 0,74 \\
\hline Brasil & 0,34 & 0,35 & 0,50 & 0,50 & 0,34 & 0,40 & 0,48 & 0,50 & 0,58 & 0,57 & 0,59 \\
\hline México & 0,53 & 0,58 & 0,57 & 0,54 & 0,58 & 0,55 & 0,56 & 0,56 & 0,57 & 0,56 & 0,53 \\
\hline
\end{tabular}

Tabla 2. Evolución de la producción de sandías de los principales países en millones de toneladas (FAO, 2014).

\begin{tabular}{|l|c|c|c|c|c|c|c|c|c|c|c|} 
& $\mathbf{2 0 0 4}$ & $\mathbf{2 0 0 5}$ & $\mathbf{2 0 0 6}$ & $\mathbf{2 0 0 7}$ & $\mathbf{2 0 0 8}$ & $\mathbf{2 0 0 9}$ & $\mathbf{2 0 1 0}$ & $\mathbf{2 0 1 1}$ & $\mathbf{2 0 1 2}$ & $\mathbf{2 0 1 3}$ & $\mathbf{2 0 1 4}$ \\
\hline China & 57,83 & 60,11 & 62,85 & 62,26 & 63,02 & 65,00 & 68,41 & 69,14 & 70,96 & 73,16 & 75,05 \\
\hline Turquía & 3,83 & 3,97 & 3,81 & 3,80 & 4,00 & 3,81 & 3,68 & 3,86 & 4,04 & 3,89 & 3,89 \\
\hline Irán & 2,53 & 3,26 & 2,87 & 3,33 & 2,57 & 3,07 & 3,47 & 3,19 & 3,22 & 3,69 & 3,57 \\
\hline Brasil & 1,72 & 1,64 & 1,95 & 2,09 & 2,00 & 2,07 & 2,05 & 2,20 & 2,08 & 2,16 & 2,17 \\
\hline Egipto & 1,59 & 1,87 & 2,03 & 1,91 & 1,49 & 1,65 & 1,64 & 1,51 & 1,87 & 1,59 & 2,01 \\
\hline Uzbekistán & 0,57 & 0,62 & 0,74 & 0,84 & 0,98 & 1,07 & 1,18 & 1,29 & 1,42 & 1,56 & 1,70 \\
\hline Argelia & 0,72 & 0,86 & 0,79 & 0,82 & 0,84 & 1,03 & 1,22 & 1,29 & 1,50 & 1,50 & 1,61 \\
\hline EE.UU. & 1,67 & 1,74 & 1,91 & 1,69 & 1,81 & 1,76 & 1,89 & 1,69 & 1,64 & 1,64 & 1,51 \\
\hline Rusia & 0,92 & 0,96 & 0,99 & 0,85 & 1,38 & 1,42 & 1,15 & 1,57 & 1,45 & 1,42 & 1,43 \\
\hline México & 1,00 & 0,86 & 0,98 & 1,06 & 1,20 & 1,01 & 1,04 & 1,00 & 1,03 & 0,95 & 0,95 \\
\hline España & $\mathbf{0 , 8 2}$ & $\mathbf{0 , 7 2}$ & $\mathbf{0 , 7 3}$ & $\mathbf{0 , 7 9}$ & $\mathbf{0 , 7 2}$ & $\mathbf{0 , 8 5}$ & $\mathbf{0 , 7 8}$ & $\mathbf{0 , 7 7}$ & $\mathbf{0 , 8 7}$ & $\mathbf{0 , 8 7}$ & $\mathbf{0 , 8 6}$ \\
\hline India & 0,30 & 0,31 & 0,32 & 0,34 & 0,35 & 0,36 & 0,37 & 0,38 & 0,40 & 0,40 & 0,41 \\
\hline
\end{tabular}

Melón

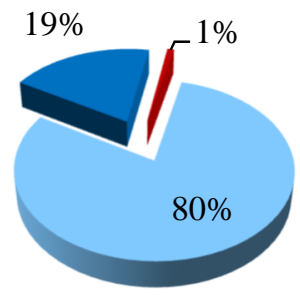

\section{Sandía}

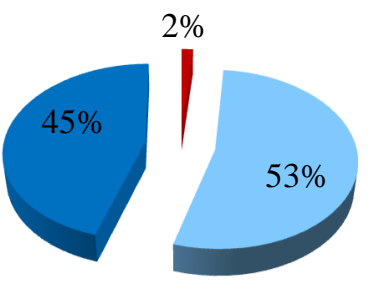


(MAPAMA, 2015).

La mayor parte del melón español procede de regadío al aire libre, seguida a distancia por regadío en cultivo protegido (Figura 2). En la actualidad el cultivo en secano representa el $1 \%$ de la producción. Hay que tener en cuenta, no obstante, que el rendimiento en cultivo de regadío al aire libre y en secano son un $17 \%$ y $83 \%$ inferior al correspondiente cultivo en regadío protegido. Así, en realidad la superficie cultivada de melón en secano es del 4\%. La mayor producción y área plantada de melón, con un 34\% del total de España, se encuentra en Castilla-La Mancha seguida por Murcia y Andalucía (Figura 3). En CastillaLa Mancha, Ciudad Real aporta casi 6800 hectáreas (218.000 toneladas) y Toledo aproximadamente 1.000 hectáreas (MAPAMA, 2015). Esta región produce principalmente melones de estación en verano. La región de Andalucía, principalmente Almería, se caracteriza por agricultura intensiva en cultivo sin suelo en invernaderos, con 2100 hectáreas en 2014 (Baixauli \& Aguilar, 2002; MAPAMA, 2015; Tolón and Lastra, 2010).

En cuanto a las exportaciones de melón, España exporta más del 50\% de su producción (Tabla 2 y 3) siendo Murcia la comunidad autónoma con mayor volumen de exportación, seguida de Andalucía y la Comunidad Valenciana (Tabla 3).
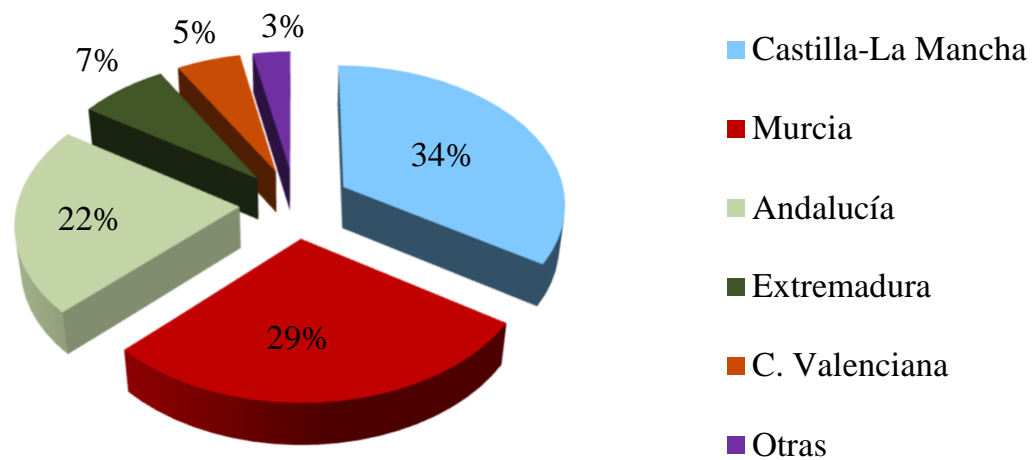

Figura 3. Producción de melones en miles de toneladas de las comunidades más relevantes de España en el año 2014 (MAPAMA, 2015).

En el caso de la sandía, la producción en secano también es testimonial, representando un $2 \%$ de la producción total. Sin embargo, al contrario de lo que ocurre en melón, la producción del cultivo en regadío al aire libre o protegido es similar (Figura 2). Andalucía produce más del $60 \%$ de la producción española de sandías seguida por Murcia y CastillaLa Mancha (Figura 4). El 98\% de esta producción es de regadío (Figura 2), y casi el 50\% se realiza como cultivo protegido (MAPAMA, 2015). 
De la misma forma, Andalucía es el mayor exportador seguida de Murcia y Comunidad Valenciana (Tabla 3).
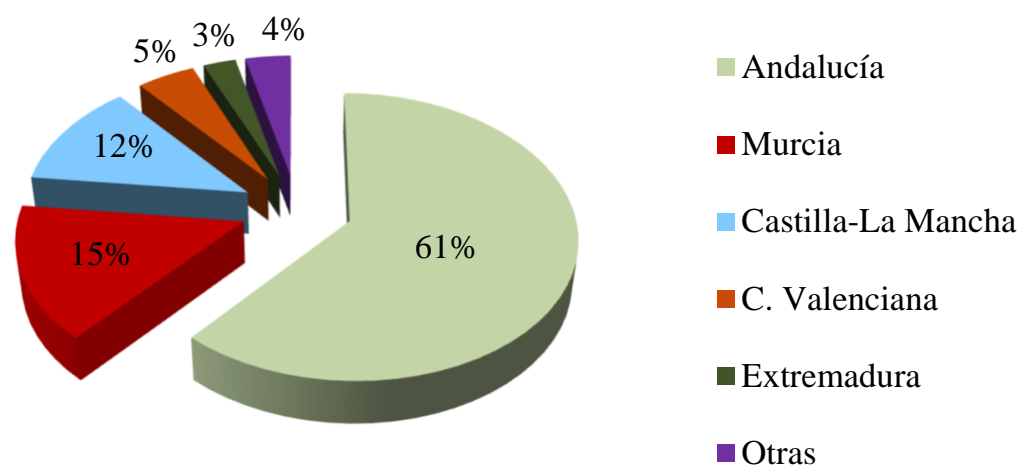

Figura 4. Producción de sandías en miles de toneladas de las comunidades más relevantes de España en el año 2014 (MAPAMA, 2015).

Tabla 3. Exportaciones de melón y sandía (en miles de toneladas) de las principales Comunidades Autónomas de España (FEPEX, 2016).

\begin{tabular}{|c|c|c|c|c|c|c|c|c|c|c|}
\hline & \multicolumn{2}{|c|}{2012} & \multicolumn{2}{|c|}{2013} & \multicolumn{2}{|c|}{2014} & \multicolumn{2}{|c|}{2015} & \multicolumn{2}{|c|}{2016} \\
\hline & Melón & Sandía & Melón & Sandía & Melón & Sandía & Melón & Sandía & Melón & Sandía \\
\hline Andalucía & 91,5 & 227,2 & 98,2 & 245,1 & 79,6 & 244,9 & 90,6 & 302,3 & 94,7 & 346,2 \\
\hline Murcia & 204,3 & 125,6 & 196,0 & 144,7 & 209,8 & 144,3 & 230,5 & 160,9 & 227,4 & 161,5 \\
\hline C. Valenciana & 98,3 & 135,6 & 82,2 & 130,4 & 72,1 & 127,6 & 69,2 & 141,8 & 70,2 & 152,3 \\
\hline Castilla - La Mancha & 19,6 & 5,3 & 13,5 & 6,1 & 16,5 & 7,3 & 16,3 & 12,2 & 20,8 & 21,3 \\
\hline Cataluña & 13,2 & 11,6 & 13,1 & 10,0 & 15,7 & 9,7 & 18,5 & 12,1 & 16,7 & 11,2 \\
\hline Madrid & 2,3 & 1,6 & 5,8 & 4,7 & 8,3 & 22,9 & 8,0 & 23,7 & 12,5 & 24,7 \\
\hline Extremadura & 1,6 & 0,8 & 0,8 & 0,8 & 1,0 & 0,9 & 0,1 & 0,4 & 0,4 & 1,4 \\
\hline TOTAL & 431,7 & 508,2 & 410,5 & 542,2 & 404,1 & 558,2 & 434,7 & 654,6 & 444,4 & 719,4 \\
\hline
\end{tabular}

\subsection{Tendencias productivas y de mercado}

El cultivo de melón y sandía ha ido cambiando a lo largo de los años. En el caso del melón, las variedades tradicionales de "larga vida" han caído prácticamente en el olvido debido al cultivo forzado y la globalización del mercado agrícola, con importaciones desde el hemisferio sur. En el caso de la sandía, el consumidor asocia el concepto de variedad tradicional a una sandía redonda y oscura, cuando en realidad en el levante español las 
variedades más antiguas eran las conocidas como "melonas", con frutos oblongos y rayados.

Ambas especies están no obstante arraigadas en el subconsciente popular y aunque la diversidad existente es muy amplia, su aprovechamiento en el mercado nacional está muy limitado. En el caso del melón, muchas variedades tradicionales han caído en el olvido. Al caso de las variedades "larga vida" como podría ser el Tendral, se suman otras de coloración externa blanca o amarilla. La mayor parte del mercado nacional se asocia al "Piel de sapo". Otras variedades como las pertenecientes al grupo cantalupensis no han cuajado en el mercado nacional, aunque se cultiven para exportación. En el caso de la sandía, a pesar de existir variedades de coloración interna amarilla o naranja, el color rojo oscuro es el predeterminado por el consumidor nacional. Si bien la forma redonda y el color verde oscuro uniforme ha sido durante muchos años el preferido, el desarrollo de las primeras variedades triploides conllevó la aceptación de los patrones rayados utilizados para diferenciarlas de los polinizadores diploides. Estas variedades, en la actualidad disponibles en color uniforme verde oscuro casi negro, dominan el mercado.

En ambas especies se trabaja por ofrecer frutos de menor tamaño. En el caso del melón esta tendencia pasa por ofrecer frutos de variedades como "Galia", "Amarillo" o "Cantalupo" de menor tamaño. En el caso de la sandía, las variedades "mini" han experimentado cierto auge. No obstante, la tendencia de mercado más importante en ambas especies, como ya se ha comentado, es la comercialización de fruto cortado que permite el consumo de piezas pequeñas en las que se aprecie la calidad de la fruta.

Desde el punto de vista del cultivo, la tendencia en España ha consistido en priorizar el cultivo en regadío. Como se ha comentado, en ambas especies el cultivo en secano es muy reducido y el cultivo en regadío al aire libre representa en la actualidad la mayor parte de la producción. Uno de los problemas que han tenido que solventar ambas especies es su elevada susceptibilidad a enfermedades del suelo. De esta forma, en muchas zonas la subsistencia del cultivo ha pasado por la necesidad de emplear plantas injertadas sobre patrones resistentes. El injerto, no obstante, puede tener una repercusión negativa sobre la calidad que hay que vigilar. Así, en Europa hemos asistido desde los años 90 a una evolución desde una agricultura de cantidad hacia una agricultura de calidad (Bouma et al., 1998), por lo que la calidad no debe descuidarse. 


\section{El uso del injerto en melón y sandía}

El injerto es una técnica efectiva muy utilizada actualmente en hortalizas para la lucha contra patógenos (hongos, nematodos y otros) evitando el contacto de la variedad sensible con el agente patógeno. Su interés también se extiende a la hora de favorecer cierto nivel de tolerancia a factores abióticos tales como: temperatura, salinidad, sequía, etc. (Davis et al., 2008).

Esta técnica puede definirse como la unión de dos porciones de tejido vegetal de modo que se unan, crezcan y se desarrollen como una sola planta (Hartmann et al., 1997). Es un método en el que una porción de tejido procedente de una planta (llamada variedad o injerto) se une sobre otra ya asentada, el patrón (también llamado portainjerto o pie). La variedad comercial sensible se injerta sobre una planta resistente, que puede ser un ancestro o simplemente una planta de otra especie o género de la misma familia.

En el caso del melón y la sandía el injerto cobra especial interés en la lucha contra los nematodos y la fusariosis, una de las principales enfermedades en estos cultivos. Esta enfermedad está causada por Fusarium oxysporum Schltdl. (f. sp. melonis en melón y niveum en sandía) que infectan suelos de algunas zonas de producción intensiva en España como Almería (Camacho, 2000). El efecto se produce desde el trasplante, al entrar en contacto con los hongos del suelo, produciendo daños visibles (amarrillo-pardo) a nivel de hojas y tallos, conduciendo a la necrosis del sistema vascular y muerte de la planta; y por consecuencia una disminución drástica de la producción de los cultivos.

El injerto cobra especial importancia desde que se prohibió en Europa el uso de bromuro de metilo (Miguel et al., 2004). Este era el mejor método de desinfestación del suelo disponible, pero fue prohibido en Europa a partir del 2005 según el Reglamento (CE)

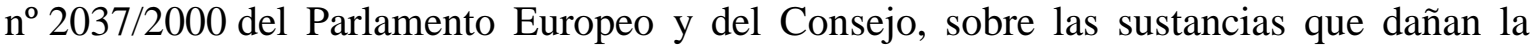
capa de ozono. Se trata no sólo de una alternativa eficiente, sino que además es completamente respetuosa con el medioambiente.

No es por tanto de extrañar que su uso en estos cultivos se haya popularizado rápidamente. Así, en un informe realizado sobre la situación del injerto en España, se puede ver el creciente uso de esta técnica desde mediados de los 90, principalmente en la Comunidad Valenciana (Figura 5) y en avance en otras zonas de gran producción (Hoyos, 2007). 


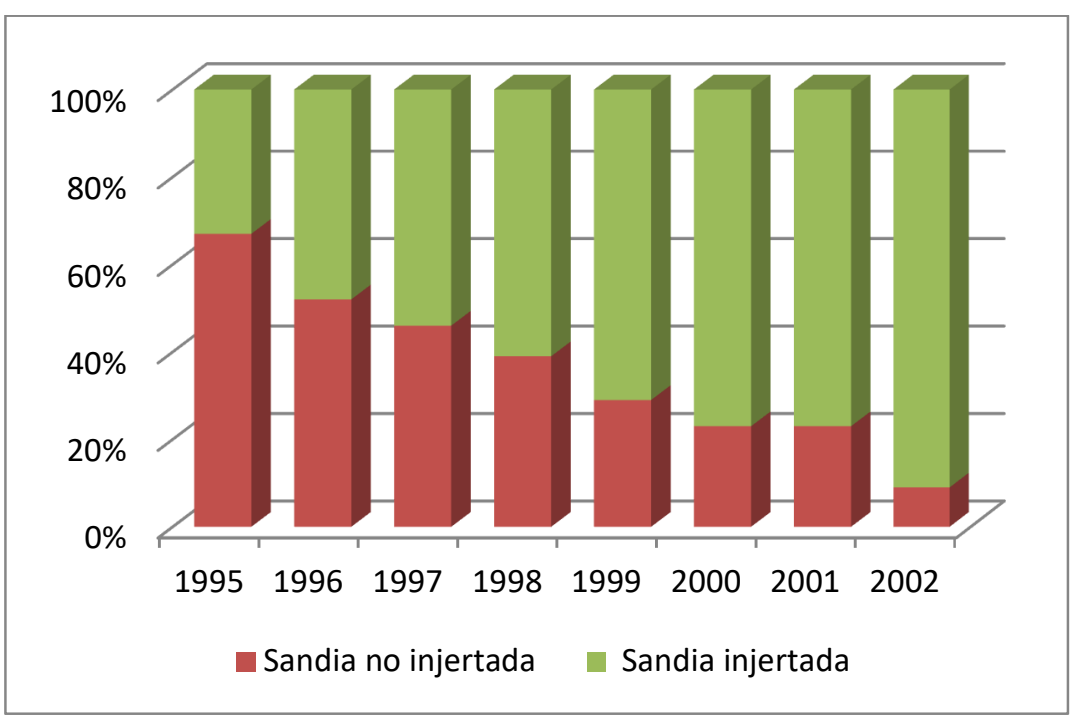

Figura 5. Evolución del porcentaje de sandía injertada frente a la sin injertar en semilleros de la Comunidad Valenciana (adaptado de Hoyos, 2007).

Desde sus primeros usos en cucurbitáceas en Europa (principios de los años 60 para melón y finales de los 70 en sandía) los métodos de injerto han ido evolucionando, aunque el más utilizado para estos cultivos es el de "aproximación” (descrito por Chavagnat, 1972; Suzuki, 1972), en el que durante la fase de unión, se conservan los sistemas radiculares tanto del patrón como de la variedad (Figura 6). Se puede considerar que este método presenta ventajas como facilidad, rapidez y, sobre todo, asegura la supervivencia de ambas partes frente a condiciones ambientales fluctuantes. Este método está ampliamente extendido en España y ya existen empresas que comercializan las plantas injertadas para su trasplante (Miguel, 2009).
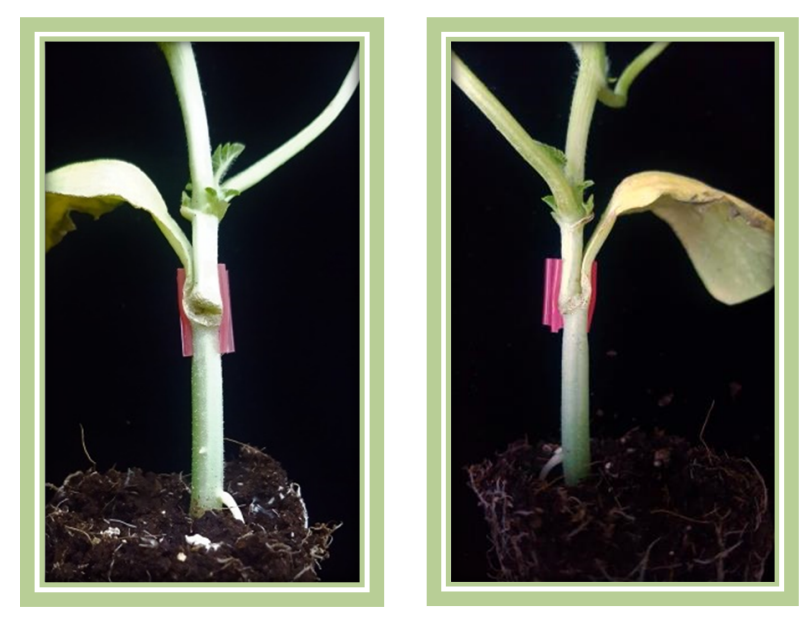

Figura 6. Ejemplo de injerto. 
Rouphael et al. (2010) revisaron el efecto del injerto sobre distintos aspectos agronómicos y de calidad de hortalizas. En el caso del melón y la sandía, el injerto puede conllevar en estas especies un aumento de la productividad tanto por el aumento del tamaño de los frutos como por el número de frutos por planta. Respecto al aspecto exterior no sólo destaca el mencionado aumento de tamaño, sino la menor incidencia de defectos externos. Desde el punto de vista de la calidad interna, en ambas especies el injerto suele aumentar la firmeza de la carne. Esto no tiene porque representar un aspecto negativo, aunque sí lo sería un aumento de la fibrosidad de la carne, observado en algunos casos (Muramatsu, 1981). El injerto también afecta a la acumulación de sólidos solubles totales y más concretamente de azúcares en el fruto. No obstante, los resultados contradictorios obtenidos en los estudios que analizan este efecto parecen sugerir que es muy dependiente de la combinación portainjerto-variedad y del ambiente en que se cultiva.

Los efectos del injerto sobre un aspecto clave de la calidad organoléptica como es el aroma han sido muy poco estudiados, fundamentalmente por la dificultad que entraña su análisis. En el caso del melón, Condurso et al. (2012) observaron que los frutos de melón "Proteo" (var. reticulatus) obtenidos a partir de plantas injertadas en portainjertos de híbridos de calabaza (Cucurbita maxima × Cucurbita moschata) y melón resistente ("Energia"), mostraron producciones superiores a los controles no injertados, mientras que los perfiles de volátiles fueron similares y los carotenos aumentaron en diversidad y cantidad en los portainjertos de híbridos de calabaza; sin embargo en casi todos los injertos la acumulación de los compuestos claves como ethyl 2-methylbutanoate y ethyl butanoate se vio disminuida. Por su parte, un estudio realizado con los mismos portainjertos pero en melones var. inodorus presentaron una reducción de la acumulación de (Z)-3-nonen-1-ol y (Z)-6-nonenal de entre un 20-60\% y 8-45\% respectivamente; aunque debido al aumento en la producción y tamaño de los frutos, se recomienda el uso de dos portainjertos híbridos de calabaza: RS841 y Polifemo (Verzera et al., 2014).

En el caso de la sandía, Petropoulos et al. (2014) observaron que los frutos provenientes de plantas injertadas en híbridos de calabaza y Lagenaria sp. presentaban similares ventajas en la productividad, pero un aumento en la síntesis de los aldehídos (E,Z)-2,6-nonadien-1al y (E)-2-nonenal que se relacionan al aroma característico de pepino, lo cual en cierta medida puede ser perjudicial en el aroma de sandía.

En conclusión, se puede decir que el injerto en estos cultivos es beneficioso en varios aspectos productivos, pero la calidad organoléptica de los frutos debe ser evaluada y 
comprobada para cada combinación de patrón injerto para encontrar la que aporte la resistencia requerida sin afectar las características de calidad de la variedad objetivo. Además, debido a que las características aromáticas de estos frutos se están convirtiendo en un distintivo de calidad, es fundamental disponer de métodos de evaluación y selección precisos y suficientemente rápidos.

El uso de determinaciones sensoriales en programas de mejora resulta prácticamente inviable por la gran cantidad de muestras a evaluar y la complejidad del proceso. Esto hace que haya una tendencia creciente en el uso de determinaciones analíticas que resultan más precisas y objetivas.

\section{CALIDAD}

La calidad se define como la propiedad o conjunto de propiedades inherentes a algo, que permiten juzgar su valor (RAE, 2014). Normalmente se habla de productos de calidad cuando se quiere manifestar que tiene una elevada calidad, por lo que el concepto acaba implicando a aquellas características que definen el grado de excelencia o superioridad de un producto (Kader, 2002).

Como objetivo comercial, la calidad en el sector hortofrutícola se dirige a explotar aquellas características que consigan satisfacer al cliente. Desde este punto de vista, cuando el consumidor tiene que tomar la decisión de comprar algún producto, la calidad se acaba convirtiendo en un complejo de muchos atributos, algunos percibidos por el cliente en momento de la compra (calidad organoléptica externa) y otros que pueden ser percibidos durante el consumo (calidad organoléptica interna) o no ser percibidos (calidad interna funcional).

\subsection{Calidad organoléptica}

La calidad organoléptica hace referencia a las características del alimento que percibe el consumidor a través de los sentidos. Es por tanto un concepto complejo y variable. Por ejemplo, en tienda, el alimento se percibe fundamentalmente por su aspecto exterior percibido a través de la vista (color, forma, uniformidad, etc.), por el aroma externo 
percibido por el olfato o por la dureza percibida por el tacto. En este momento prima por tanto la calidad organoléptica externa. Durante el consumo, se percibe la calidad organoléptica interna. De nuevo participa la vista, evaluando aspectos como el color interno o la estructura del alimento, el tacto, esta vez a través de las sensaciones del masticado (arenosidad, harinosidad, crujiente, jugosidad, etc.) y el aroma, añadiéndose el gusto (dulzor, acidez, amargor, etc.) y el aroma interno percibido también retronasalmente.

Como se ha comentado, la apariencia es la primera impresión que el consumidor tiene del producto y la que lo llevará a decidir si compra un producto u otro. Puesto que una gran parte de los consumidores toma dicha decisión de compra dentro del propio supermercado (López, 2003), los atributos de calidad externa se convierten en un aspecto importante a tener en cuenta a la hora de definir la calidad de un producto.

En muchas frutas y hortalizas, aspectos externos como color, tamaño, etc. dan una idea de la madurez del producto. En el caso de melón y sandía, la apariencia externa no permite diferenciar con eficacia el estado de madurez en el que se encuentra el fruto. De ahí, la creciente tendencia a encontrar en el mercado melón y sandía presentados como fruto cortado (Beaulieu et al., 2004; Fonseca et al., 2004) en los que el consumidor es capaz de apreciar el estado de la pulpa y tener más información sobre la madurez del producto que va a adquirir (Figura 7). Además, al tratarse de frutos de gran tamaño, otra ventaja de este tipo de presentación es que permite comprar menos cantidad de producto.

El éxito de esta estrategia comercial y la gran demanda de fruto cortado han favorecido que el concepto haya ido evolucionando, llevando al desarrollo de productos en los que el melón o la sandía se presentan envasados en atmósfera modificada tras ser pelados y troceados, listos ya para comer (Torres \& Miquel, 2003).

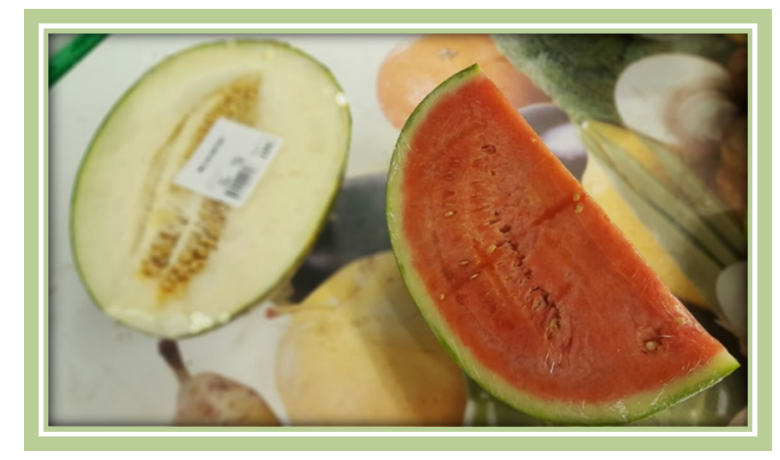

Figura 7. Oferta de frutos de melón y sandía cortados en punto de venta. 
A diferencia de la calidad organoléptica externa, la calidad organoléptica interna no puede ser juzgada por el consumidor hasta que el fruto no es consumido y por tanto no condiciona la decisión de compra. Sin embargo, estos atributos son los que van a determinar que el consumidor siga comprando el producto o no lo haga, ya que confirman o desmienten las apreciaciones percibidas con la calidad externa.

La calidad organoléptica interna viene determinada por atributos relacionados con el aspecto interno (espesor de la pulpa y de la corteza, tamaño de la cavidad placentaria, color de la pulpa) y su composición bioquímica (contenido en azúcares y aromas) (Monforte \& Alvarez, 2006; Pretel et al., 1993).

En el caso de melón y sandía, la calidad organoléptica interna viene definida principalmente por su sabor y aroma (Fallik et al., 2001; Kourkoutas et al., 2006; Shalit et al., 2001). El sabor de estos frutos guarda una relación directa con el contenido de azúcares (Yamaguchi et al., 1977), estando matizado en el caso de algunos melones por la presencia de ácidos orgánicos. Tanto los melones de tipo dulce como las sandías tienen un bajo contenido en ácidos orgánicos, siendo los más abundantes en fruto maduro los ácidos cítrico y málico (Chachin \& Iwata, 1988; Liu et al., 2012; Wang et al., 1996).

En cuanto a los azúcares, los principales presentes en estos frutos son glucosa, fructosa y sacarosa (Lester, 2008; Yoo et al., 2012). En melón, la sacarosa es el azúcar mayoritario en fruto maduro de las variedades consideradas ricas en azúcar, mientras que glucosa y fructosa destacan en las variedades menos dulces (Stepansky et al., 1999b). En el caso de la sandía, algunos genotipos acumulan una mayor cantidad de sacarosa mientras que en otros el azúcar mayoritario es la fructosa. Al contrario de lo que parece ocurrir en melón, el fruto podría emplear la fructosa y glucosa además de la sacarosa como azúcares de reserva, lo que implica que las sandías con mayor acumulación de azúcares totales no serían necesariamente aquellas que acumulan más sacarosa (Yativ et al., 2010). Tanto en melón como en sandía, en las primeras etapas del crecimiento del fruto, destaca la presencia de fructosa y glucosa, mientras que la sacarosa comienza a acumularse en etapas más avanzadas de la maduración. Este aumento en el contenido en sacarosa no se realiza utilizando las hexosas existentes, sino que la nueva sacarosa se suma a la fructosa y glucosa ya presentes. Así, la sacarosa provendría de hidratos de carbono transportados desde las hojas a los frutos durante el proceso de maduración (Hubbard et al., 1989). Para explicar el proceso, Gross \& Pharr (1982) propusieron que la estaquiosa y rafinosa se traslocarían desde las hojas al fruto y en el pedúnculo se hidrolizarían generando galactosa, 
que entonces se emplearía para sintetizar sacarosa. Esta hipótesis partía de la ausencia de estaquiosa y rafinosa en el mesocarpo del fruto. Sin embargo, en estudios posteriores sí que se han detectado en este tejido del fruto, por lo que se propuso que este metabolismo no tendría lugar en el pedúnculo sino en el fruto y que la hidrólisis sería muy rápida, explicando los bajos contenidos de estos azúcares (Hubbard et al., 1989).

La variación del contenido en azúcar en estas especies está estrechamente relacionada con la actividad enzimática implicada en el metabolismo de los azúcares. En este proceso participan invertasas y enzimas implicadas en la síntesis de sacarosa: Sacarosa Sintasa, SS y Sacarosa Fosfato Sintasa, SPS (Yativ et al., 2010). En el caso de las invertasas, se ha observado una correlación negativa entre la concentración de estas enzimas y la de sacarosa en los frutos. Por el contrario, las enzimas implicadas en la síntesis de sacarosa aumentan su actividad en las etapas en las que se observa mayor acumulación de sacarosa. En el caso de la sandía, la enzima SS, que presenta una baja actividad durante los primeros meses de crecimiento aumenta de forma importante hacia el final de la maduración en genotipos con alto contenido en sacarosa, mientras que en los genotipos en los que la concentración final de sacarosa es baja, se aprecia un descenso de su actividad durante todo el proceso de crecimiento. En el caso de la SPS, en las variedades consideradas como pobres en sacarosa, la actividad del enzima es menor que en las de alta concentración de éste azúcar. En ambos tipos de variedades, la actividad de la enzima se mantiene constante durante el crecimiento del fruto (Yativ et al., 2010).

En el caso del melón, es necesario un descenso de la actividad de la invertasa para que se produzca la acumulación de sacarosa pero, puesto que el descenso de la actividad de esta enzima va asociada a un aumento de la maduración, la acumulación final de sacarosa parece estar marcada en mayor medida por la actividad de la enzima SPS (Hubbard et al., 1989).

Pero, como se ha indicado anteriormente, la calidad organoléptica del melón y la sandía no sólo viene dada por el contenido en azúcares, sino que los compuestos volátiles responsables del aroma también jugarían un papel importante.

En el caso del melón, se aprecian diferencias importantes en dichos volátiles en función de si se trata de frutos climatéricos o no climatéricos. Los frutos climatéricos son aquellos que presentan un aumento en la actividad respiratoria asociada a la maduración del fruto debida a un aumento de la producción autocatalítica de etileno (Bower et al., 2002). Estos frutos son capaces de continuar su maduración después de ser separados de la planta siempre que 
hayan alcanzado su madurez fisiológica mientras seguían unidos a ésta; mientras que los no climatéricos sólo continúan con su proceso de maduración si se encuentran unidos a la planta, no presentando apenas variación en la síntesis de etileno durante este proceso.

Dentro de los compuestos volátiles que determinan el aroma de melón en frutos climatéricos en estado maduro destacan los ésteres, seguidos de cantidades mucho más pequeñas de aldehídos y alcoholes de cadena media, terpenos y lactonas (Bauchot et al., 1998; Shalit et al., 2001). Los frutos no climatéricos son menos aromáticos que los anteriores, presentando mucha menos proporción de ésteres y destacando los alcoholes de cadena media y corta y los aldehídos alifáticos (Saftner et al., 2006; Shalit et al., 2001). Además, se ha observado que la porción volátil en los frutos climatéricos varía mucho su composición en función de si los frutos están en estado maduro e inmaduro; mientras que en melones no climatéricos se observan sólo pequeñas variaciones en la porción volátil en función del estado de madurez del fruto (Shalit et al., 2001).

La formación de los aldehídos deriva de la degradación de ácidos grasos, formándose los alcoholes a partir de aldehídos o por degradación de amino ácidos. En el caso de los ésteres, la formación se da por esterificación de alcoholes y ácidos carboxílicos, estando implicadas las alcohol aciltransferasas (ATT). Estas enzimas catalizan la transformación al alcohol correspondiente a partir de acetil-CoA (Shalit et al., 2001). En el caso del melón, se han detectado hasta cuatro miembros de este grupo de enzimas, estando presentes en melones aromáticos en estado maduro, mientras que no se ha detectado actividad de estas enzimas en melones inmaduros o en tipos no aromáticos (Katzir et al., 2006; Yariv et al., 2004). En melones con acumulación de carotenoides pueden detectarse compuestos como la $\beta$-ionona provenientes de la degradación de los mismos (Burger et al., 2006).

En sandía, los principales componentes de la fracción volátil son aldehídos y alcoholes con cadenas de nueve átomos de carbono, encontrándose también otros compuestos como cetonas, lactonas y furanos (Beaulieu \& Lea, 2006; Pino et al., 2003; Yajima et al., 1985). Al igual que en los frutos no climatéricos de melón, en sandía también se ha comprobado la falta de actividad de alcohol aciltransferasas (Ueda et al., 1997). Teniendo en cuenta el alto contenido en carotenoides, en esta especie la ruta de degradación de carotenoides aporta una fuente de volátiles muy importante en la definición del aroma de la sandía. Una ruta muy similar a la presente en tomate (Lewinsohn et al., 2005).

Hasta el momento se han encontrado más de 300 compuestos que pueden intervenir en el aroma a melón y alrededor de 80 en el caso de la sandía. En ambos casos alrededor de 63 
compuestos definen en gran medida el perfil de volátiles de ambas especies (Tabla 4).

Tabla 4. Principales compuestos orgánicos aromáticos presentes en muestras de melón y sandía (Aubert \& Pitrat, 2006; Beaulieu, 2006a, 2006b; Beaulieu \& Grimm, 2001; Beaulieu \& Lea, 2006; Homatidou et al., 1992; Jordán et al., 2001; Kourkoutas et al., 2006; Liu et al., 2012; Obando-Ulloa et al., 2008; Obando-Ulloa \& Ruiz, 2010; Perry et al., 2009; Saftner et al., 2006; Verzera et al., 2011).

\begin{tabular}{|c|c|c|c|c|c|}
\hline & COMPUESTOS & № CAS & & COMPUESTOS & № CAS \\
\hline 1 & Methyl butyrate & $623-42-7$ & 32 & E-2-octenal & $2548-87-0$ \\
\hline 2 & Methyl 2-methylbutyrate & $868-57-5$ & 33 & (Z)-6-nonenal & $2277-19-2$ \\
\hline 3 & 2-methylpropyl acetate & $110-19-0$ & 34 & Octyl acetate & $112-14-1$ \\
\hline 4 & Ethyl butyrate & $105-54-4$ & 35 & (E,E)-2,4-heptadienal & $881-39-5$ \\
\hline 5 & Ethyl 2-methylbutyrate & $7452-79-1$ & 36 & Decanal & $112-31-2$ \\
\hline 6 & Butyl acetate & $123-86-4$ & 37 & (E,E)-2,4-hexadienoic acid, ethyl ester & $2396-84-1$ \\
\hline 7 & Hexanal & $66-25-1$ & 38 & Benzaldehyde & $100-52-7$ \\
\hline 8 & (Z,Z)-3,6-nonadienol & $53046-97-2$ & 39 & (E)-2-nonenal & $18829-56-6$ \\
\hline 9 & Propyl butanoate & $105-66-8$ & 40 & Linalool & $78-40-6$ \\
\hline 10 & Ethyl pentanoate & $539-82-2$ & 41 & 1-octanol & $111-87-5$ \\
\hline 11 & Butyl isobutyrate & $97-87-0$ & 42 & Ethyl 3-(methylthio)propanoate & $13327-56-5$ \\
\hline 12 & Isobutyl butyrate & $539-90-2$ & 43 & (E,Z)-2,6-nonadienal & $557-48-2$ \\
\hline 13 & Ethyl (E)-2-butenoate & $623-70-1$ & 44 & $\beta$-ciclocytral & $5392-40-5$ \\
\hline 14 & Amyl acetate & $123-86-4$ & 45 & Phenylacetaldehyde & $122-78-1$ \\
\hline 15 & Heptanal & $111-71-7$ & 46 & 1-nonanol & $143-08-8$ \\
\hline 16 & Methyl hexanoate & $106-70-7$ & 47 & 2-hydroxybenzaldehyde & $90-02-8$ \\
\hline 17 & Eucalyptol & $470-82-6$ & 48 & Z-3-nonen-1-ol & $10340-23-5$ \\
\hline 18 & Butyl butanoate & $109-21-7$ & 49 & $\mathrm{E}, \mathrm{E}-2,4$-nonadienal & $5910-87-2$ \\
\hline 19 & Ethyl hexanoate & $123-66-0$ & 50 & Z-6-nonen-1-ol & $35854-86-5$ \\
\hline 20 & 1-pentanol & $71-41-0$ & 51 & Benzyl acetate & $140-11-4$ \\
\hline 21 & Isoamyl butanoate & $106-27-4$ & 52 & 1-decanol & $112-30-1$ \\
\hline 22 & Hexyl acetate & $142-92-7$ & 53 & $\mathrm{E}, \mathrm{Z}-2,6$-nonadien-1-ol & $28089-72-9$ \\
\hline 23 & Octanal & $124-13-0$ & 54 & E,E-2,4-decadienal & $25152-84-5$ \\
\hline 24 & (Z)-3-hexen-1-ol acetate & $3681-71-8$ & 55 & Phenethyl acetate & $103-45-7$ \\
\hline 25 & E-2-heptenal & $18829-55-5$ & 56 & Geranylacetone & $689-67-8$ \\
\hline 26 & Ethyl heptanoate & $106-30-9$ & 57 & Guaiacol & $90-05-1$ \\
\hline 27 & 6-methyl-5-hepten-2-one & $110-93-0$ & 58 & Benzyl alcohol & $100-51-6$ \\
\hline 28 & 1-hexanol & $111-27-3$ & 59 & 2-phenylethanol & $60-12-8$ \\
\hline 29 & Heptyl acetate & $112-06-1$ & 60 & $\beta$-ionone & $14901-07-6$ \\
\hline 30 & Z-3-hexen-1-ol & $928-96-1$ & 61 & Phenol & $108-95-2$ \\
\hline 31 & Nonanal & $124-19-6$ & 62 & Eugenol & $97-53-0$ \\
\hline
\end{tabular}




\subsection{Calidad nutricional y funcional}

La calidad nutricional de un alimento está relacionada con la capacidad que éste tiene para aportar al organismo la energía y nutrientes necesarios para el buen funcionamiento del mismo, mientras que la calidad funcional contempla también la presencia de compuestos bioactivos que sin ser nutrientes ayudan a prevenir enfermedades. En ambos casos, se trata de una calidad no percibida, ya que el consumidor cuando compra el fruto o lo ingiere desconoce si realmente tiene una elevada calidad nutritiva o funcional. Es decir, hace un acto de fe respecto a la publicidad que se haga de las variedades compradas (Grunert et al., 2000).

El melón y la sandía son frutos compuestos principalmente por agua (92 y 95\% respectivamente) y una cantidad variable de azúcares dependiendo de las variedades (6 y $4.5 \%$ respectivamente) Se trata de frutos de bajo contenido calórico, con un contenido graso inferior al $0.5 \%$. Además, también presentan cierta cantidad de minerales (principalmente potasio) y vitaminas (Tabla 5). En el caso del melón, podemos encontrar cantidades importantes de ácido ascórbico y ácido fólico (Lester, 2008). En sandía también se puede encontrar ácido ascórbico (Yoo et al., 2012), aunque en menor cantidad que en el melón.

Más allá del contenido en vitaminas, en los frutos se puede encontrar otro tipo de compuestos bioactivos, en general de gran poder antioxidante, que pueden contribuir a prevenir enfermedades, principalmente cardiovasculares y degenerativas en el organismo (Kohlmeier et al., 1997; Martí et al., 2016; Van Duyn \& Pivonka, 2000). Son estos compuestos los que determinan la calidad funcional de los frutos de melón y sandía entre los cuales destacan, los carotenoides.

El $\beta$-caroteno es el principal carotenoide presente en melón de pulpa naranja, encontrando también luteína en menor cantidad (Laur \& Tian, 2011). En el caso de la sandía, en los frutos de pulpa roja destacan licopeno, fitoeno y $\beta$-caroteno, encontrando también menores cantidades de fitoflueno, $\zeta$-caroteno y luteína; mientras que en sandías de pulpa naranja destaca el $\beta$-caroteno y, en sandías de pulpa amarilla, violaxantina y luteoxantina (Liu et al., 2012).

Por otro lado, dentro de la calidad funcional de la sandía, cabe destacar la citrulina, un aminoácido presente en distintas variedades de este fruto (Rimando \& Perkins-Veazie, 
2005). La citrulina participa en el metabolismo de la arginina, un aminoácido esencial en el caso de niños y esencial condicional si se trata de adultos, en concreto en casos de traumatismo, quemadura, resección del intestino delgado o deficiencia renal. De hecho, este aminoácido tiene un papel importante en funciones renales, pulmonares, cardiovasculares, digestivas e inmunes (Flynn et al., 2002). Numerosos estudios han relacionado el aumento de arginina en plasma con el consumo de sandía (Collins et al., 2007; Mandel et al., 2005).

Tabla 5. Composición de melón y sandía en 100 g de porción comestible (adaptado de Moreiras et al., 2013).

\begin{tabular}{|c|c|c|}
\hline Componente & Melón & Sandía \\
\hline Agua $\left(g_{\left.100 g^{-1}\right)}\right.$ & 92.4 & 94.6 \\
\hline Proteínas (g 100g ${ }^{-1}$ ) & 0.6 & 0.4 \\
\hline Calorías (Kcal 100g $\mathrm{g}^{-1}$ ) & 28 & 21 \\
\hline Grasas $\left({\left.\mathrm{g} 100 \mathrm{~g}^{-1}\right)}^{-1}\right.$ & 0.28 & 0.43 \\
\hline Hidratos de carbono $\left(\mathrm{g}^{100 \mathrm{~g}^{-1}}\right)$ & 6.0 & 4.5 \\
\hline Fibra $\left(\mathrm{g} 100 \mathrm{~g}^{-1}\right)$ & 1.0 & 0.5 \\
\hline Potasio (mg 100g $\mathrm{g}^{-1}$ ) & 320 & 120 \\
\hline Hierro (mg 100g-1) & 0.21 & 0.30 \\
\hline Sodio (mg $100 \mathrm{~g}^{-1}$ ) & 14 & 4 \\
\hline Fósforo (mg 100g-1) & 18.0 & 5.5 \\
\hline Magnesio $\left(\mathrm{mg}^{\left.100 \mathrm{~g}^{-1}\right)}\right.$ & 17 & 11 \\
\hline Calcio $\left(\mathrm{mg} 100 \mathrm{~g}^{-1}\right)$ & 14 & 7 \\
\hline Vitamina $C\left(\mathrm{mg} 100 \mathrm{~g}^{-1}\right)$ & 25 & 5 \\
\hline Vitamina A: eq. Retinol ( $\mu \mathrm{g} 100 \mathrm{~g}^{-1}$ ) & 5.4 & 33.0 \\
\hline Tiamina (vit. B1) (mg $\left.100 \mathrm{~g}^{-1}\right)$ & 0.04 & 0.02 \\
\hline Riboflavina (vit. B2) (mg $100 \mathrm{~g}^{-1}$ ) & 0.02 & 0.02 \\
\hline Niacina (vit. B3) (mg 100 $\mathrm{g}^{-1}$ ) & 0.5 & 0.3 \\
\hline Ácido fólico $\left(\mathrm{mg} 100 \mathrm{~g}^{-1}\right)$ & 17 & 2 \\
\hline Carotenoides (eq. de caroteno) $\left(\mu \mathrm{g} 100 \mathrm{~g}^{-1}\right.$ ) & 669.3 & 151.4 \\
\hline
\end{tabular}




\section{EVALUACIONES DE LAS CARACTERÍSTICAS DE CALIDAD ORGANOLÉPTICA}

\subsection{Análisis Sensorial}

La mejor forma de valorar la calidad organoléptica es sin duda el análisis sensorial. Se trata de un conjunto de técnicas que pretende medir la respuesta del hombre ante un alimento determinado, percibida a través de los sentidos, reduciendo al máximo influencias externas (Lawless \& Heymann, 2010).

Uno de los principales problemas que representa el análisis sensorial deriva de las limitaciones de los paneles de cata. Estos pueden estar formados por panelistas entrenados, capaces de percibir diferencias mínimas en el sabor y de dar valoraciones coherentes (según su grado de entrenamiento), o por paneles de consumidores, que sin ser tan precisos pueden dar una idea del grado de aceptabilidad del producto en el público en general. En cualquier caso, los análisis sensoriales se ven limitados por la cantidad de muestras que puede procesar un panel sin llegar a saturarse y además, incluso en paneles entrenados, el panelista acaba representando la mayor fuente de variación (Bucheli et al., 1999).

Por ello, cuando se trata de valorar las diferencias en el perfil aromático de un gran número de muestras no es realista recurrir a paneles de cata, ya que por su coste y limitada capacidad de análisis sería impensable. Esta es una situación típica de un programa de mejora genética, en el que se tienen que fenotipar cientos o miles de muestras en poco tiempo. En muchos casos, este tipo de análisis se aborda en etapas finales de programas de mejora para realizar una selección final entre un número reducido de materiales precomerciales. Pero quizá para entonces sea demasiado tarde. De hecho, por ejemplo en tomate se ha comprobado que una de las principales razones de la pérdida de calidad organoléptica en las variedades modernas ha residido en no tener en cuenta el perfil aromático durante los programas de selección. De esta forma, aunque se ha seleccionado por la cantidad de azucares y ácidos, se han perdido alelos favorables a la acumulación de volátiles derivados del metabolismo de los carotenoides, volátiles que a su vez intensifican la percepción del dulzor (Tieman et al., 2017).

Una forma de soslayar estas limitaciones sería seleccionar atributos sensoriales 
indirectamente a partir de otras variables, por ejemplo de composición analítica, que tengan una elevada correlación con las sensoriales y que permitieran una evaluación y selección en grandes cantidades de muestras de forma objetiva.

En este contexto, es necesario contar con medidas analíticas objetivas y precisas que correlacionen con la percepción sensorial y que nos permita continuar con la selección de materiales de calidad, procesando un elevado número de muestras. Varios trabajos se han publicado en melón y sandía a este respecto. En el caso del melón, Beaulieu et al. (2004) relacionaron el estado de madurez (medido como desarrollo de la capa de abscisión) de frutos de tipo Cantalupo con atributos sensoriales relacionados con sabor, tratando de establecer el mejor estado de madurez en la recolección para la comercialización de fruta cortada. En este estudio también se correlacionaron medidas instrumentales y sensoriales de textura. Por otro lado, también se ha podido comparar la fracción volátil de tres tipos de cultivares diferentes de melón (Cantalupo, Galia y Honeydew) medida por medio de microextracción en fase sólida (SPME) con atributos de aroma y sabor medidos por un panel de cata. De esta manera se pudo relacionar los principales atributos de sabor y aroma de melón tipo Honeydew con volátiles específicos, siendo más complicado de conseguir en el caso de melón tipo Cantalupo (Kourkoutas et al., 2006). En el caso de la sandía, Pardo et al. (1997) correlacionaron determinaciones analíticas de firmeza, sólidos solubles, azúcares (sacarosa, fructosa y glucosa) y color con características sensoriales establecidas por un panel de consumidores que juzgaron el sabor, color y textura de los frutos.

\subsection{Determinaciones analíticas}

Como se ha indicado anteriormente, a pesar que el análisis sensorial es la mejor manera de evaluar la calidad organoléptica de los frutos, los problemas derivados de su uso han hecho que en las últimas décadas se hayan desarrollado y perfeccionado nuevas técnicas para poder llevar a cabo determinaciones precisas y objetivas de los distintos compuestos implicados en este aspecto de la calidad. 


\subsubsection{Determinación de ácidos y azúcares}

La determinación de ácidos y azúcares está adquiriendo gran importancia debido a la influencia que estos compuestos tienen sobre la calidad organoléptica de frutas y hortalizas. En el caso de melón y sandía, como ya se ha indicado, de estos compuestos quien se lleva la mayor parte del peso son los azúcares (Yamaguchi et al., 1977).

La determinación de sólidos solubles por medio de la refractometría es una forma rápida y sencilla de expresar (como ${ }^{\circ}$ Brix) el contenido de estos compuestos en conjunto. Esta medida presenta una alta correlación entre los valores medidos y la concentración de azúcares, pero hay que tener presente que se trata sólo de una aproximación, pudiéndose encontrar frutos de melón con iguales contenidos en ${ }^{\circ}$ Brix pero con grandes diferencias en la concentración de azúcares totales (Stepansky et al., 1999a).

La tendencia actual es el uso de técnicas que permitan cuantificar de forma precisa cada uno de los principales azúcares y ácidos implicados en el sabor de melón y sandía, como la cromatografía líquida de alta resolución (HPLC) (Stepansky et al., 1999b; Villanueva et al., 2004; Yativ et al., 2010; Yoo et al., 2012) o la electroforesis capilar zonal (CZE) (Cebolla-Cornejo et al., 2012; Kolayli et al., 2010).

Estos métodos se emplean para determinar el contenido en los principales azúcares individuales relacionados con la percepción del dulzor, que como ya se ha comentado, en ambas especies son la glucosa, la fructosa y la sacarosa. Dado que cada azúcar posee un poder edulcorante distinto, en ocasiones se matiza la aportación de cada uno a través de la variable equivalentes de sacarosa que se obtiene sumando la concentración de cada azúcar por su poder edulcorante referido a la sacarosa, es decir, por 0,74 en el caso de la glucosa, 1,73 en el caso de la fructosa y 1 en el caso de la sacarosa (Koehler \& Kays, 1991).

Las técnicas analíticas anteriormente descritas han permitido realizar estudios de la acumulación de dichos azúcares en melón y sandía con relación al estado de madurez (Wang et al., 1996; Yativ et al., 2010), partes del fruto (Hubbard et al., 1989; Weidong et al., n.d.), genotipos (Stepansky et al., 1999b; Yoo et al., 2012), la influencia del injerto (Rouphael et al., 2010) y otros factores agronómicos. 


\subsubsection{Determinación y cuantificación de compuestos orgánicos volátiles}

Al estudiar los compuestos volátiles en frutas y hortalizas es importante conocer algunas características de estos analitos en el aroma (Flath et al., 1981). Generalmente estos compuestos suelen estar en concentraciones bajas en la fracción volátil de los alimentos, por lo que su análisis requiere una etapa de pre-concentración. La fracción volátil está constituida generalmente por un gran número de componentes de diferentes pesos moleculares y naturaleza química, cuyas concentraciones pueden variar en un intervalo muy amplio (ppt - ppm). Aunque, en el caso de sandía y melón, son compuestos de bajo peso molecular (Tabla 4).

Por otro lado, no existe relación directa entre la concentración de los compuestos volátiles y su influencia en el aroma, ya que los límites de detección por el ser humano de cada uno de ellos son variables. Por lo tanto, los compuestos más abundantes en una muestra no tienen por qué ser los que contribuyan más al aroma de la misma.

Teniendo en cuenta estas razones, el análisis de la fracción volátil se puede resumir en 3 etapas: 1- liberación, extracción y concentración de los compuestos volátiles de la muestra; 2- fraccionamiento de los diferentes componentes; 3-identificación y cuantificación de los analitos.

\subsubsection{Extracción y concentración}

Los principales métodos de extracción pueden agruparse en dos grandes grupos:

\section{1) Los que no realizan concentración:}

\section{$\underline{\text { Inyección directa }}$}

Esta técnica, fue una de las primeras que se utilizó para el análisis de compuestos volátiles en sistemas cromatográficos gaseosos con columnas empaquetadas. Su utilidad se limita a compuestos termo-resistentes como los ácidos grasos volátiles que deben ser vaporizados en una soporte inerte (lana de vidrio) o compuestos que luego de una extracción líquidolíquido sencilla pueden ser inyectados directamente (Cottyn \& Boucque, 1968; Morales et 
al., 1992). En la actualidad, la inyección directa es cada vez menos utilizada por su baja sensibilidad y por el marcado "efecto memoria" que produce debido a que los analitos son inyectados junto con otros compuestos interferentes; esto hace necesario una continua limpieza del sistema entre muestra y muestra para que los datos no se distorsionen al analizar muchas muestras. Posteriormente se desarrolló la inyección directa de grandes volúmenes con el uso de un inyector denominado vaporizador de temperatura programada (PTV) que permite inyectar mayor volumen de muestra y hace posible obtener resultados comparables con algunas técnicas de pre-concentración, pero generalmente también se produce un aumento del efecto matriz (Engewald et al., 1999; Villén et al., 1995).

\section{$\underline{\text { Espacio de cabeza estático (SHS) }}$}

Otra forma de analizar la fracción volátil, consiste en colocar la muestra en un recipiente (vial) cerrado, donde el sólido o líquido alcanza el equilibrio con su fase vapor a una temperatura determinada, de la cual se extrae una alícuota (normalmente $1 \mathrm{~mL}$ de mezcla aire - vapor) que será analizada por cromatografía.

Las desventajas de este método son: su baja sensibilidad y su alto contenido en agua en la fase vapor, por lo que no es apto para análisis de trazas o compuestos poco concentrados y con baja presión de vapor (poco volátiles).

A pesar de esto, algunos sistemas de olfatómetros electrónicos utilizan esta modalidad de extracción de volátiles, ya que sus sensores presentan una sensibilidad suficiente para discriminar entre muestras de diferentes madurez y variedad en frutas; y en algunos casos obtener métodos cuantitativos para compuestos volátiles al relacionarnos con cromatografía de gases (Du et al., 2010; Lin \& Zhang, 2016).

\section{2) Los que realizan concentración:}

En la actualidad este grupo de técnicas de extracción tienen mayor relevancia ya que permiten el análisis de compuestos que presentan concentraciones bajas, incluso a nivel de trazas (Bicchi et al., 2008; Sides et al., 2000; Snow \& Slack, 2002) 


\section{Extracción con solventes}

La extracción con solventes es una de las técnicas más simples y relativamente eficientes de aislamiento de compuestos orgánicos utilizada hasta el presente (Homatidou et al., 1992). Con frecuencia, esta técnica no logra concentrar lo suficiente los compuestos de interés, por lo que se emplea el evaporador rotatorio a vacío para alcanzar los niveles de cuantificación.

Generalmente este tipo de técnicas son poco específicas para los compuestos volátiles y junto a ellos también se extraen compuestos de polaridad y características similares que dificultan posteriormente su separación e identificación. Además, existe una tendencia creciente a reducir el uso de solventes orgánicos, ya que son perjudiciales para la salud humana, generan residuos contaminantes y encarecen los análisis.

\section{Destilación}

La destilación puede clasificarse en simple y fraccionada, dependiendo del número de platos teóricos que presente la columna y de si en la mezcla existen compuestos con puntos de ebullición muy diferentes o similares, respectivamente. Otro tipo de destilación muy utilizada para la extracción de aceites esenciales y perfumes es la destilación por arrastre con vapor que utiliza el vapor de agua para arrastrar los compuestos volátiles de una muestra vegetal sin calentarla directamente (Ruberto et al., 2008). Este tipo de extracción se ha mejorado y sustituido por el uso de la destilación a vacío para compuestos que se ven más afectados por la temperatura (Hiatt et al., 1994). En cualquier caso, al utilizar calor para la evaporación de la mezcla de partida, esta técnica tiene la desventaja de degradar muchos compuestos termolábiles del aroma y formar artefactos indeseables que no estaban presentes en la muestra original (Sides et al., 2000).

\section{$\underline{\text { Espacio de cabeza dinámico (DHS) }}$}

El ejemplo más utilizado en este tipo de extracción es la Purga y Trampa (P\&T). Esta técnica en sus inicios fue aplicada a la determinación de gases disueltos en muestras acuosas (Swinnterton et al., 1962) y consiste en arrastrar los volátiles de la muestra ("purga") con un gas inerte, normalmente $\mathrm{N}_{2}$, a un flujo controlado para que pasen a través de una trampa donde quedan retenidos. Posteriormente los analitos son desorbidos de la fase sólida ("trampa") por medio de solventes o por desorción térmica (TDU). Si la 
desorción se realiza con solventes, se debe optimizar el volumen de elución para que no queden analitos retenidos en la fase estacionaria y además es necesaria una preconcentración (por corriente de $\mathrm{N}_{2}$ o evaporación al vacío) para obtener una sensibilidad adecuada. La purga y trampa ha sido utilizada en sus diferentes variantes por varios autores para la determinación de compuestos volátiles en diferentes frutas y hortalizas (Beltran et al., 2006; Kanavouras et al., 2005; Leffingwell et al., 2015; Sales et al., 2017).

Esta técnica posee ciertas ventajas, como la posibilidad de extraer varias muestras simultáneamente ya que los cartuchos utilizados no son costosos. Además, la variedad de fases sólidas existente la convierten en una herramienta versátil. Sin embargo, si no se cuenta con el dispositivo de desorción térmica es necesario el uso de solventes orgánicos que la convierten en una técnica menos respetuosa con el medio ambiente, así como más costosa.

\section{Microextracción en fase sólida (SPME)}

En 1989, Janusz Pawliszyn y colaboradores de la Universidad de Waterloo, desarrollaron una técnica libre de solventes que permite el muestreo, extracción y concentración de compuestos volátiles (Pawliszyn, 2000, 1997). Esta consiste en la extracción de los compuestos con una delgada fibra de sílice fundida recubierta por una fina capa de fase sólida adsorbente, que puede variar según la naturaleza del analito. Debido a su fragilidad, el dispositivo de extracción cuenta con una aguja de acero hueca que protege la fibra de posibles roturas, mientras no está en uso.

En ocasiones a la muestra se le añaden sales (p.ej. cloruro de sodio) y/o cierta cantidad de disolvente no volátil (p.ej. propilenglicol) para potenciar la volatilidad de los analitos hacia el espacio de cabeza. Además debe estar termostatizada y homogeneizada uniformemente. Una vez alcanzado el equilibrio en estas condiciones, se introduce la fibra en el espacio de cabeza dejándola al descubierto y permitiendo que los volátiles se adsorban sobre el recubrimiento. Tras un tiempo determinado se retira la fibra colocándola directamente en el puerto inyector del cromatógrafo de gases para su desorción térmica.

Esta técnica de extracción es una de las más utilizadas en la actualidad en una gran diversidad de matrices gaseosas y líquidas (Bicchi et al., 2008). Al no utilizar solventes orgánicos, logra pre-concentrar los analitos aumentando la sensibilidad considerablemente, disminuye los costes del análisis y no produce residuos contaminantes. Sin embargo, 
también presenta una serie de desventajas: existe un número limitado de recubrimientos comerciales; solo pueden ser sumergidas en solventes acuosos; las fibras presentan una vida útil relativamente corta y con el tiempo la fase estacionaria comienza a degradarse (sangrado) interfiriendo en el análisis; la adsorción competitiva de los volátiles y, en algunos casos, saturación de la fina cubierta adsorbente, lo que podría no representar realmente la concentración relativa de los volátiles (Stashenko \& Martínez, 2007).

Extracción por adsorción en barra agitadora (SBSE) o extracción por adsorción en espacio de cabeza (HSSE)

Este tipo de extracción se desarrolló inicialmente para la determinación de compuestos orgánicos en muestras acuosas, denominada extracción por adsorción en barra agitadora (SBSE). Consiste en una pastilla magnética de vidrio que posee un revestimiento adsorbente (por ej. polidimetilsiloxano) que extrae los compuestos orgánicos presentes en la muestra.

Sin embargo, algunos estudios han usado esta misma barra con un dispositivo que la ubica en el espacio de cabeza, de forma similar a la SPME pero con la ventaja adicional de una mayor superficie y capacidad adsorbente (Bicchi et al., 2002; Tienpont et al., 2000). Además, debido a su mayor capacidad de adsorción, concentra la muestra aproximadamente 100 veces más que la SPME. A pesar de ello presenta algunas desventajas: un alto efecto matriz, la necesidad de un control minucioso de las condiciones para una buena repetitividad y en algunos casos los recubrimientos disponibles limitan la naturaleza de los analitos estudiados (Camino-Sánchez et al., 2014).

\subsubsection{Separación, identificación y cuantificación}

Una vez obtenida la mezcla concentrada de volátiles es necesario separar, identificar y cuantificar los analitos presentes en la misma. La cromatografía de gases (GC) es hoy en día la metodología más estudiada para la determinación de compuestos volátiles, utilizada en gran diversidad de matrices (Beaulieu, 2006a; Biniecka \& Caroli, 2011; Katayama \& Kaneko, 1969; Kemp, 1975; Verzera et al., 2011).

La cromatografía de gases se basa en la distribución de los componentes de una mezcla entre dos fases inmiscibles, la fase estacionaria (líquido o sólido) y la fase móvil (gas). Se 
cree que esta técnica fue propuesta por Archer J.P. Martin y Richard L.M. Synge, quienes observaron la posibilidad de remplazar la fase móvil de la cromatografía líquida por un gas (Martin \& Synge, 1941). Debido a la alta difusividad que presentan los gases en los solutos, en comparación con los líquidos, el equilibrio del proceso cromatográfico sería más rápido, con una mayor eficiencia de las columnas y un menor tiempo de análisis. El desarrollo de sistemas informáticos ha permitido el diseño de analizadores cada vez más complejos, llegando a la diversidad de cromatógrafos comerciales de la actualidad, que aún conservan la idea inicial de la separación de compuestos volátiles.

Estos instrumentos se componen de tres partes diferenciadas:

1) El sistema de inyección.

2) La columna (fase estacionaria) ubicada dentro de un horno termostatizado, donde los compuestos son separados según su punto de ebullición y afinidad química con la fase estacionaria.

3) Detector y sistema de registro.

Los compuestos son arrastrados por un gas inerte (generalmente helio o nitrógeno) que circula desde el inyector hacia el detector. La versatilidad en la determinación de compuestos de diferente naturaleza se debe a la diversidad de columnas cromatográficas (empaquetadas, tubulares y capilares) y detectores presentes en el mercado.

El detector más común por su universalidad y bajo coste, es el detector de ionización de llama (FID). Se trata de un detector útil para llevar a cabo análisis rutinarios, pero la escasa información que proporciona sobre los analitos hace que la identificación de los compuestos no conocidos sea ineficiente (Sides et al., 2000). Por ello, en la mayoría de las determinaciones de volátiles, se utiliza la espectrometría de masas (MS) para la identificación y cuantificación precisa de los mismos (Biniecka \& Caroli, 2011; Yajima et al., 1985). Este dispositivo fragmenta los analitos que ingresan desde columna cromatográfica por medio de ionización electrónica o química, generando un conjunto característico de iones que son transportados por campos magnéticos hacia el analizador, donde son separados según su relación masa/carga $(\mathrm{m} / \mathrm{z})$. Finalmente, en el detector se cuantifica la abundancia relativa de dichos iones, dando lugar al espectro de masas que constituye la fragmentación patrón de cada compuesto. En la actualidad existe una amplia diversidad de espectrómetros de masas dependiendo de sus componentes (fuentes de iones, analizador y detector) pero la mayor diferencia entre ellos radica en el corazón del equipo, o sea, el analizador de masas. Existen diversos tipos de analizadores de masas con 
fundamentos analíticos diferentes entre los cuales se encuentran: cuadrupolo (quadrupole; Q), trampa de iones (ion-trap, IT), tiempo de vuelo (time-of-flight, TOF), sector magnético (magnetic sector), ciclotrón (ion cyclotron resonance, ICR) y los híbridos que combinan a los anteriores.

Los MS más utilizados por su sencillez, diseño compacto, versatilidad y precio, son aquellos que presentan fuente de ionización por impacto electrónico (EI) e ionización química (CI), con analizador cuadrupolo o trampa de iones y detector electromultiplicador (Burinsky, 2006).

\subsubsection{Olfatómetros electrónicos}

Las técnicas cromatográficas actuales permiten identificar los componentes volátiles involucrados en el aroma de diferentes muestras y cuantificar de forma precisa su contenido, de modo que se pueda conocer qué volátiles contribuyen al aroma de un fruto y la cantidad de cada uno de ellos en esa mezcla. Sin embargo, resulta complicado extraer conclusiones sobre cómo sería percibida esta fracción volátil por los sentidos humanos a partir del conocimiento de contenido individual de estos compuestos, ya que en esa percepción están involucrados no solo una gran cantidad de compuestos con umbrales de detección diferentes sino que, además, la interacción de diferentes compuestos entre sí la condiciona en gran medida.

Por ello, una de las soluciones que se ha intentado aportar ha sido complementar las técnicas cromatográficas con la detección olfatométrica (GCO). En esta técnica, los compuestos previamente separados toman dos vías diferentes. Por un lado se cuantifica la abundancia de los compuestos; por otro, un operario entrenado realiza una determinación olfativa del eluyente humedecido basada en descriptores del aroma. De esta manera se logra relacionar la cuantificación de los compuestos volátiles de la muestra con descriptores específicos para aquellos que sean detectables por el olfato humano (Campo et al., 2005; Ferreira et al., 2009; Jordán et al., 2001). Se podría considerar que el GCO es el nexo entre las técnicas cromatográficas tradicionales y el análisis sensorial, sin embargo, el GCO continúa siendo una técnica de separación que no aporta información sobre la percepción global del perfil aromático. Además, al no ser posible su automatización, no es una técnica rápida y sencilla a la hora de procesar un gran número de muestras. 
En este contexto, surge la necesidad de desarrollar una técnica que permita simular las respuestas de los receptores presentes en la nariz humana, obteniendo un resultado de la fracción volátil más objetivo que el proporcionado por un panel de cata, sin necesidad de determinar la composición química propiamente dicha. De esta forma se podría determinar si un alimento es similar a otro en lo que concierne a su fracción aromática.

Otro planteamiento adoptado ha sido el uso de analizadores de gases o "Sniffers". Los primeros analizadores de gases se desarrollaron con el objetivo de determinar bajos niveles de oxígeno, altas concentraciones de gases combustibles y presencia de monóxido de carbono o gas metano en el aire de los túneles de la industria minera. Entre los años 1926 1965 los doctores Oliver Johnson y Phil Williams fueron claves en el desarrollo de los primeros aparatos de detección de gases denominados "J-W Sniffer" (por las iniciales de sus creadores) aunque en la actualidad no se consideran verdaderos olfatómetros electrónicos ya que estos analizadores constaban de un solo sensor basado en la combustión catalítica. Este sensor estaba constituido de un pequeño compartimento con un filamento catalítico (usualmente de platino o paladio) que reaccionaba a los gases combustibles aumentando su temperatura y como consecuencia su resistencia. A partir de la medida de resistencia comparada con la de un filamento de referencia (no catalítico) se obtenía la medida indirecta del contenido del gas.

Posteriormente surgieron los primeros trabajos publicados donde K. Persaud y G. Dodd de la Universidad de Warwick (Gran Bretaña) estudiaron cómo los sensores semiconductores de óxidos metálicos (MOS) se relacionan con los receptores del olfato humano (Persaud \& Dodd, 1982). En la misma época surge el concepto de olfatómetro o nariz electrónica, como un instrumento que consta de una matriz de sensores físico-químicos capaces de reaccionar a diferentes tipos de compuestos y un sistema apropiado de reconocimiento de patrones, capaz de reconocer olores simples o complejos (Gardner \& Bartlett, 1999).

De la misma forma que el olfato humano percibe el estímulo de los componentes volátiles del aroma y el cerebro relaciona este estímulo con experiencias anteriores guardadas en la memoria, las narices electrónicas utilizan el patrón de respuesta de los sensores para discriminar entre un grupo de muestras diferentes (Figura 8). 


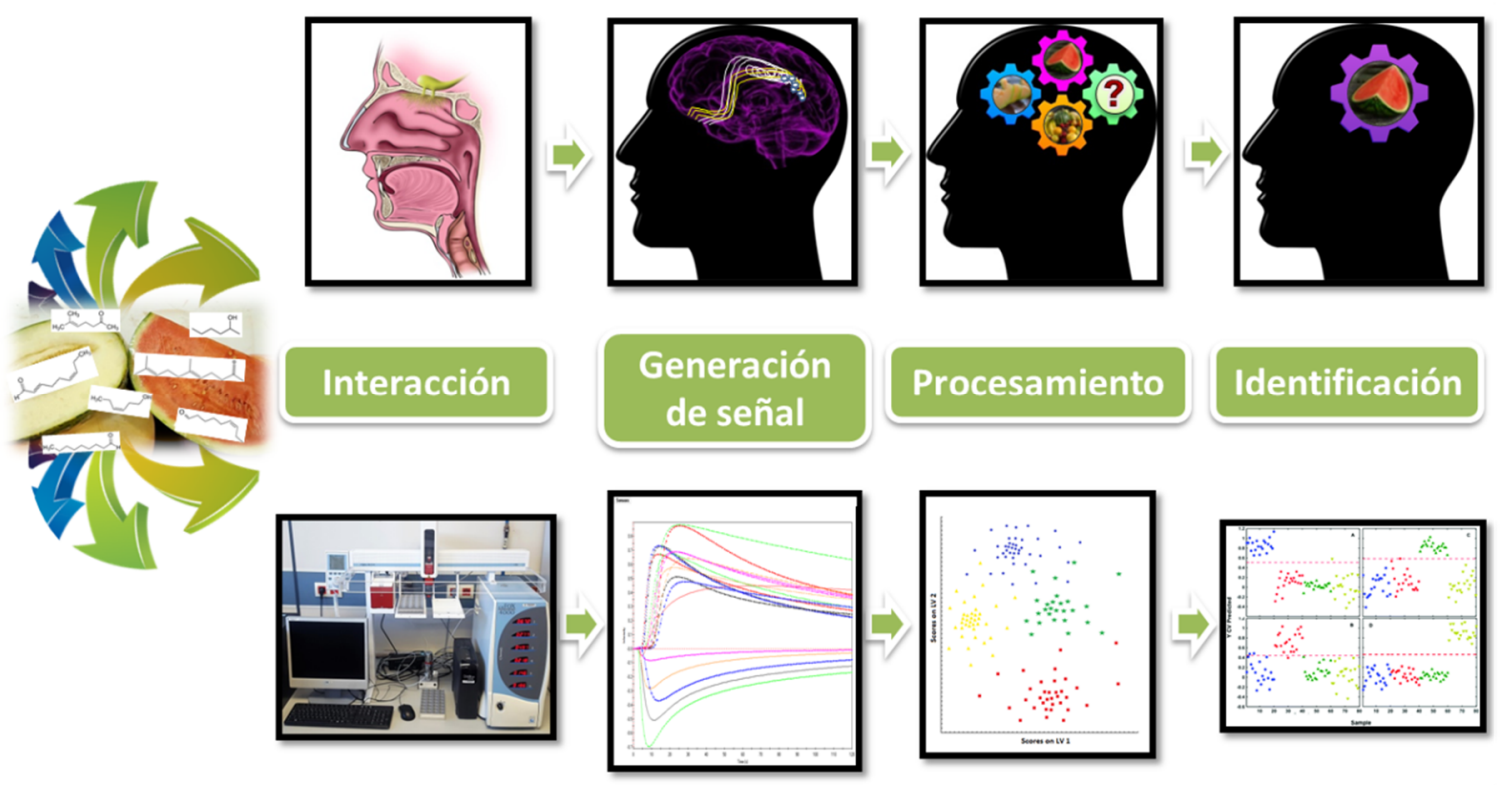

Figura 8. Esquema comparativo del funcionamiento de la nariz humana versus la "nariz electrónica". Modificadas de Bort et al., 2017.

Estos equipos proporcionan aproximaciones válidas a la hora de separar grupos de muestras con características aromáticas diferentes, asemejándose a los paneles de cata pero sin contar con la precisión de cuantificación de las técnicas cromatográficas (Gardner \& Persaud, 2001).

La ventaja principal de los olfatómetros electrónicos radica en que las determinaciones no requieren de extracción o pre-tratamiento de la muestra, por lo que los tiempos de evaluación son menores que para otras técnicas. La respuesta dada por los sensores no se corresponde con cada uno de los compuestos por separado, sino que proporciona una idea de la fracción volátil global, una "huella electrónica" única para cada muestra. Esto le otorga cierta similitud con los paneles de cata, frente a los que presenta una serie de ventajas: mayor objetividad, mayor capacidad de análisis sin tener que dejar tiempos de descanso (por saturación de receptores de los sentidos de los jueces del panel), mayor sensibilidad de discriminación y la posibilidad de construir librerías de perfiles aromáticos a largo plazo. Todo esto hace que sea una herramienta muy útil en las evaluaciones de material vegetal en planes de mejora genética.

Sin embargo, a pesar de su rapidez y sencillez para los análisis de rutina, presenta una serie de desventajas que con el paso del tiempo se han ido resolviendo. Algunos estudios han demostrado que los sensores electrónicos experimentan una disminución de la sensibilidad 
debido a la presencia de vapor de agua en el aire (Gardner \& Persaud, 2001), sus respuestas presentan deriva por varios factores propios del sensor o el ambiente (Holmberg \& Artursson, 2003) y que en algunos la vida útil es corta (Nanto \& Stetter, 2003).

Se han dado múltiples soluciones a la deriva o variabilidad en la respuesta de los sensores intra e interdía. En nuestro grupo se investigó la posibilidad de corrección de deriva utilizando patrones sintéticos y regresión parcial mínimo cuadrática para determinar la parte de la señal que recogen los sensores que solo es función del tiempo y no de tipo de muestra y de este modo poder "limpiar" la señal eliminando esta deriva (Valcárcel, 2009). Esta metodología presenta la ventaja de permitir la creación de mapas de similitud de perfiles electrónicos a largo plazo para la comparación de un gran número de genotipos de diferentes especies en estudio.

Hoy en día se pueden encontrar gran variedad de equipos, que presentan diferencias en cuanto a los sensores utilizados y las aplicaciones a las que van destinados (Tabla 6).

Tabla 6. Características de diferentes olfatómetros electrónicos comerciales.

\begin{tabular}{|c|c|c|}
\hline Empresa & Producto & Características \\
\hline $\begin{array}{l}\text { Airsense Análisis } \\
\text { GmbH }\end{array}$ & PEN2, PEN3 & $\begin{array}{l}\text { Sensores MOS. Equipo portátil usado en } \\
\text { control de calidad, control ambiental y de } \\
\text { seguridad }\end{array}$ \\
\hline AlphaMos & $\begin{array}{l}\text { Fox4000, } \\
\alpha-\text {-Gemini } \\
\text { Heracles }\end{array}$ & $\begin{array}{l}\text { Sensores MOS. Equipos de laboratorio } \\
\text { automatizados. } \\
\text { Heracles ofrece un análisis cualitativo y semi- } \\
\text { cuantitativo de muestras gracias a la técnica } \\
\text { GC-MS*. }\end{array}$ \\
\hline Cyrano Science Inc. & Cyranose 320 & $\begin{array}{l}32 \text { sensores } \mathrm{CP} \text {. Equipo portátil para } \\
\text { determinación de campo. }\end{array}$ \\
\hline $\begin{array}{l}\text { Electronic Sensor } \\
\text { Technology }\end{array}$ & $\begin{array}{l}\text { Model } 4200 \text { zNose, } \\
\text { Model } 7100 \text { Benchtop zNose. }\end{array}$ & $\begin{array}{l}\text { Sensores } \mathrm{SAW}^{*} \text {. Identifican trazas de } \\
\text { componentes orgánicos, biológicos y } \\
\text { químicos. }\end{array}$ \\
\hline INFICON & $\begin{array}{l}\text { HAPSITE® } \\
\text { Smart Plus Chemical } \\
\text { Identification System }\end{array}$ & $\begin{array}{l}\text { GC-MS portátil. Identificar y cuantificar } \\
\text { sustancias químicas industriales tóxicas y } \\
\text { agentes de amenazas químicas in situ de forma } \\
\text { más rápida y sencilla. }\end{array}$ \\
\hline $\begin{array}{l}\text { Technobiochip, } \\
\text { Universidad de Roma } \\
\text { (Tor Vergata) }\end{array}$ & LibraNose & $\begin{array}{l}\text { Sensores QCM. Equipo compacto y fácil de } \\
\text { utilizar. }\end{array}$ \\
\hline
\end{tabular}

Las aplicaciones de estos equipos varían desde el control ambiental, pasando por la seguridad (control de fugas en industria y transporte, control de contaminantes en ciudades) y el control en la calidad de diferentes productos alimenticios, sobre todo de origen agrícola (Berna \& Amalia, 2010; Moreno et al., 2009). En los últimos años existen 
una gran variedad de estudios que evalúan diversas características en frutos (diferencias genotípicas y de estado de madurez, por ejemplo) a partir de las huellas electrónicas obtenidas de la fracción volátil. Se pueden citar estudios realizados en tomate (Hernández et al., 2008; Sinesio et al., 2000), pepino (Zawirska-Wojtasiak et al., 2009), manzana (Brezmes et al., 2000; Marrazzo et al., 2005; Young et al., 1999), pera (Zhang et al., 2008), melocotón (Benedetti et al., 2008), albaricoque (Solis-Solis et al., 2007), naranja (Di Natale et al., 2001), fresas y otros frutos rojos (Du et al., 2010; Simon et al., 1996), uva (Athamneh et al., 2008), mango (Lebrun et al., 2004), banana (Llobet et al., 1999), níspero (Guarrasi et al., 2011), melón (Benady et al., 1995) y otros (Pokhum et al., 2010).

Todos los sistemas de nariz electrónica que existen actualmente en el mercado constan de tres partes, el sistema de muestreo de volátiles, el sistema de sensores que interactúan con los volátiles de la muestra y un módulo informático con diferentes herramientas quimiométricas (estadísticas) para el procesado de señal electrónica y la evaluación de los resultados.

\subsubsection{Sistema de toma de muestra}

La extracción de volátiles en espacio de cabeza estático (HS), es la técnica habitual, ya que se obtienen los compuestos libres del resto de la matriz. En el caso de los olfatómetros, las muestras son agitadas y calentadas, permitiendo la pre-concentración de los compuestos volátiles en el espacio de cabeza, que serán inyectados posteriormente a las cámaras de sensores.

\subsubsection{Sensores}

Los olfatómetros electrónicos poseen un número variable de sensores, desde dos hasta más de treinta (Marrazzo et al., 2005; Simon et al., 1996). Las diferencias entre las "narices electrónicas" radican específicamente en la naturaleza de los sensores, ya que éstos son la parte central de la olfatometría. Dichos sensores modifican sus propiedades físico-químicas al interaccionar con los compuestos volátiles, dando una respuesta que posteriormente será modificada (pre-tratamiento, previo al uso de técnicas quimiométricas) para obtener una "huella electrónica" del aroma de la muestra. 
Dependiendo del material con el que se fabrican y el funcionamiento, los sensores más utilizados se pueden clasificar en los siguientes tipos:

\section{$\underline{\text { Sensores de conductividad }}$}

\section{- Quimioresistentes}

Los sensores Semiconductores de Óxidos Metálicos (M.O.S.) fueron los más utilizados en los primeros prototipos de analizadores de gases y siguen siendo utilizados en la actualidad. Como su nombre indica son sensores fabricados con óxidos metálicos que varían su conductividad eléctrica, disminuyendo su resistencia al paso de los electrones, cuando los compuestos impactan sobre la superficie del semiconductor. Requieren altas temperaturas (entre $200-500^{\circ} \mathrm{C}$ ) para su buen funcionamiento.

Se pueden clasificar en dos tipos:

1) Tipo "n": tienen un exceso de electrones (cargas negativas) y están compuestos por óxido de estaño $\left(\mathrm{SnO}_{2}\right)$, óxido de zinc $(\mathrm{ZnO})$, óxido de hierro III $\left(\mathrm{Fe}_{2} \mathrm{O}_{3}\right)$ u óxido de wolframio $\left(\mathrm{WO}_{3}\right)$ que le confieren la propiedad de reaccionar frente a especies gaseosas reductoras como $\mathrm{H}_{2}, \mathrm{CH}_{4}, \mathrm{CO}, \mathrm{C}_{2} \mathrm{H}_{5}$ o $\mathrm{H}_{2} \mathrm{~S}$.

2) Tipo "p": los cuales tienen un exceso de cargas positivas o "huecos" que le confieren una reactividad mayor a los gases oxidantes como $\mathrm{O}_{2}, \mathrm{NO}_{2}$ y $\mathrm{Cl}_{2}$. Están compuestos de óxido de cobre $(\mathrm{CuO})$, óxido de níquel $(\mathrm{NiO})$ u óxido de cobalto (CoO).

Son sensores disponibles comercialmente, con una buena sensibilidad y tiempos de respuestas cortos (10 segundos), aunque deben ser dopados con metales como el platino $(\mathrm{Pt})$ o paladio $(\mathrm{Pd})$ y utilizar diferentes temperaturas para aumentar su selectividad a los compuestos gaseosos. La desventaja más relevante es que presentan una alta sensibilidad a la humedad, el $\mathrm{CO}_{2}$ y el etanol, por lo que no se obtienen buenos resultados cuando se utiliza como gas de arrastre aire con trazas de humedad o al analizar muestras que contienen alcohol.

Otro tipo de sensores estudiados más recientemente como alternativa a los anteriores son los Polímeros Orgánicos Conductores (C.P.). Estos son fabricados a partir de los monómeros del: pirrol (polipirrol), anilina (polianilina), acetileno (poliacetileno) o tiofeno (politiofeno). 
Dichos sensores presentan las siguientes ventajas: trabajar a temperatura ambiente, presentar mayor selectividad con alta sensibilidad, tener bajo coste y que el proceso de adsorción de los compuestos sea reversible. A pesar de esto, cuentan con la desventaja de ser sensibles a la humedad y la temperatura, por lo que la reproducibilidad baja considerablemente.

\section{- Potenciométricos}

Estos sensores son semiconductores de óxido metálico basados en efecto de campo (FET) por lo que se denominan MOSFET. Están compuestos por un metal catalizador de paladio, iridio o rodio que descansa sobre un trozo de óxido metálico aislante y un semiconductor de silicio. La polarización eléctrica producida en la interacción de las moléculas con la superficie catalítica provoca una diferencia de potencial brusca que se traduce en una caída de voltaje medible (Schaller et al., 1998). Las principales ventajas son su bajo coste, alta sensibilidad, mayor versatilidad para variar su selectividad por medio del material del catalizador y de la temperatura; pero presenta prácticamente las mismas desventajas que los sensores MOS.

\section{$\underline{\text { Sensores Gravimétricos o piezoeléctricos }}$}

Estos sensores determinan la masa de compuestos volátiles absorbidos sobre una membrana químicamente selectiva a partir del cambio en la frecuencia de oscilación de un cristal que posee propiedades piezoeléctricas. El material piezoeléctrico es un tipo de cristal que al ser estresado por medios mecánicos es capaz de adquirir una polarización eléctrica y, por lo tanto, una diferencia de potencial. Generalmente están compuestos de un disco de cristal de cuarzo $\left(\mathrm{SiO}_{2}\right)$, niobato de litio $\left(\mathrm{LiNbO}_{3}\right)$ o tantalato de litio $\left(\mathrm{LiTaO}_{3}\right)$ recubierto de una fina capa de un material térmicamente estable, capaz de capturar moléculas de la fracción volátil, como por ejemplo las porfirinas o los lípidos (Guilbault et al., 1988).

Actualmente existen dos tipos de sensores piezoeléctricos estructuralmente diferentes:

- Microbalanzas de Cristal de Cuarzo (QCM) o dispositivo de onda acústica masiva (BAW). En este tipo particular de sensor la frecuencia de oscilación (10- 
$30 \mathrm{MHz}$ en el cuarzo) viaja a través del cristal entero. Como ventaja cabe destacar su tamaño (son sensores pequeños) y su amplia versatilidad, otorgada por el recubrimiento. Sin embargo, la forma de deposición de estos recubrimientos no se ha perfeccionado y las variaciones de temperatura y humedad limitan en gran medida la reproducibilidad de las medidas.

- Dispositivo de onda acústica superficial (SAW). Su funcionamiento y estructura es muy similar al anterior con la diferencia de que las oscilaciones sólo se producen sobre la superficie del cristal. Estos son más sensibles y rápidos que los BAW por presentar una mayor frecuencia de vibración (100-1000 MHz), pero su sensibilidad sigue siendo baja en comparación a los demás sensores y no se cuenta con buenas interfaces eléctricas.

\section{$\underline{\text { Sensores Ópticos }}$}

Este tipo de sensores se basa en la medición de variaciones en las propiedades ópticas como la reflectancia, interferencia, fluorescencia, fosforescencia, quimioluminiscencia o color en presencia de compuestos volátiles activos. Algunos de estos sensores presentan una alta sensibilidad y selectividad ya que no todos los volátiles presentan este tipo de propiedades. Además, no existen problemas de interferencias electrónicas o magnéticas gracias al uso de fibra óptica con excelente aislamiento, incluso para el análisis en medios de difícil acceso. Como inconvenientes cabe destacar que la detección es más lenta, por la trasferencia de masa entre la capa sensible y el analito a determinar; y que el tiempo de vida de este tipo de sensores es bastante bajo.

\subsubsection{Métodos Quimiométricos}

Los métodos quimiométricos de evaluación de la señal electrónica obtenida en los olfatómetros se utilizan en dos etapas sucesivas, en primer lugar, se realiza un pretratamiento de la señal y posteriormente se realiza la evaluación de los resultados propiamente dichos con distintos tipos de metodologías de análisis multivariante en función de los objetivos que se persigan. 


\section{Pre-tratamiento de la señal}

Habitualmente las señales obtenidas por los sensores necesitan de algún tipo de pretratamiento para poder ser utilizados posteriormente en diversos tipos de análisis multivariante. Los principales pre-tratamientos consisten en (Gutierrez-Osuna, 2002):

- Manipulación de línea base: este pre-tratamiento consiste en sustraer (diferencia), dividir (relativo) o sustraer y dividir (fraccional) la línea base a la respuesta del sensor.

- Compresión o corrección: el objetivo es extraer de la señal de cada sensor la parte de información que más representa la variabilidad de la respuesta que caracteriza el perfil aromático de la muestra, eliminando ruido y/o deriva de señal. Este tipo de pre-tratamiento en algunos casos no se realiza si los métodos multivariantes que se emplean en el análisis de resultados utilizan alguna metodología de búsqueda de factores latentes (PCA, PLS, PLS-DA, ANN, etc.) que puede, indirectamente, realizar esta compresión o corrección. No obstante, en ciertas aplicaciones (construcción de mapas a largo plazo de huellas electrónicas) es necesario hacerlo.

- Normalización: este tipo de pre-tratamiento se utiliza principalmente para uniformizar las respuestas de los sensores (variables) con diferentes escalas.

\section{Análisis Multivariantes}

Cada sensor da lugar a uno o más parámetros medibles, como por ejemplo, intensidad máxima relativa, tiempo requerido para que el sensor alcance el máximo de intensidad, pendiente de la recta que une la intensidad máxima con el inicio de respuesta del sensor, etc. Cada uno de estos parámetros representa una variable distinta que aporta información sobre la interacción de la fracción volátil de la muestra con cada sensor. Para poder relacionar estas variables entre sí y obtener resultados interpretables sobre las características aromáticas de las muestras es necesario el uso de análisis estadísticos multivariantes.

Dentro de los análisis multivariantes el subgrupo más utilizado para los datos provenientes de olfatómetros electrónicos son las técnicas de reconocimiento de patrón de respuesta 
(PARC: pattern recognition). Aunque también algunos de ellos permiten la cuantificación indirecta de volátiles a partir de los parámetros obtenidos por los sensores.

El tipo concreto de análisis a utilizar dependerá del tipo de información que se pretenda obtener de los datos de perfiles electrónicos obtenidos. En la actualidad, los principales análisis estadísticos utilizados para procesar datos de nariz electrónica se presentan en la Figura 9, en la que se muestran dos grandes grupos en función del origen de estos (desarrollo puramente matemático o de inspiración en procesos biológicos) (Hines et al., 2003):

Métodos estadísticos clásicos, entre los cuales destacan:

- Regresión lineal múltiple (MLR),

- Análisis de componentes principales (PCA),

- Regresión de componentes principales (PCR),

- Regresión parcial por mínimos cuadrados (PLS),

- Análisis de función discriminante (DFA),

- Análisis discriminantes a partir de regresión parcial mínimo cuadrática (PLS-DA),

- Máquinas de vectores soporte (SVM),

- Análisis de clúster (CA), siendo uno de los más utilizados el análisis de vecino más próximo $(\mathrm{NN})$.

Métodos inspirados en procesos biológicos. Estos métodos se desarrollaron, inspirados en diversos procesos de tipo biológico, para obtener modelos que se adaptaran con mayor versatilidad a diferentes tipos de estructuras de datos. La mayor parte de estos métodos se idearon y se fueron refinando para imitar el modo de procesado de información que realizan las redes neuronales humanas. No obstante, no son los únicos, también existen otro tipo de métodos inspirados en procesos biológicos como aquellos que utilizan algoritmos genéticos que simulan procesos de recombinación de información para generar variabilidad como los que se producen en el ADN cromosómico durante los procesos de meiosis. Entre los métodos de este grupo, los más ampliamente utilizados son:

- Perceptrón multicapa (MLP),

- Mapas auto-organizados (SOM),

- Redes de funciones de base radial (RBF),

- Cuantificación del vector de aprendizaje (LVQ), 
- Teoría de resonancia adaptativa (ART),

- Lógica difusa en mapas ART (Fuzzy ARTMAP),

- Algoritmos genéticos (GA).

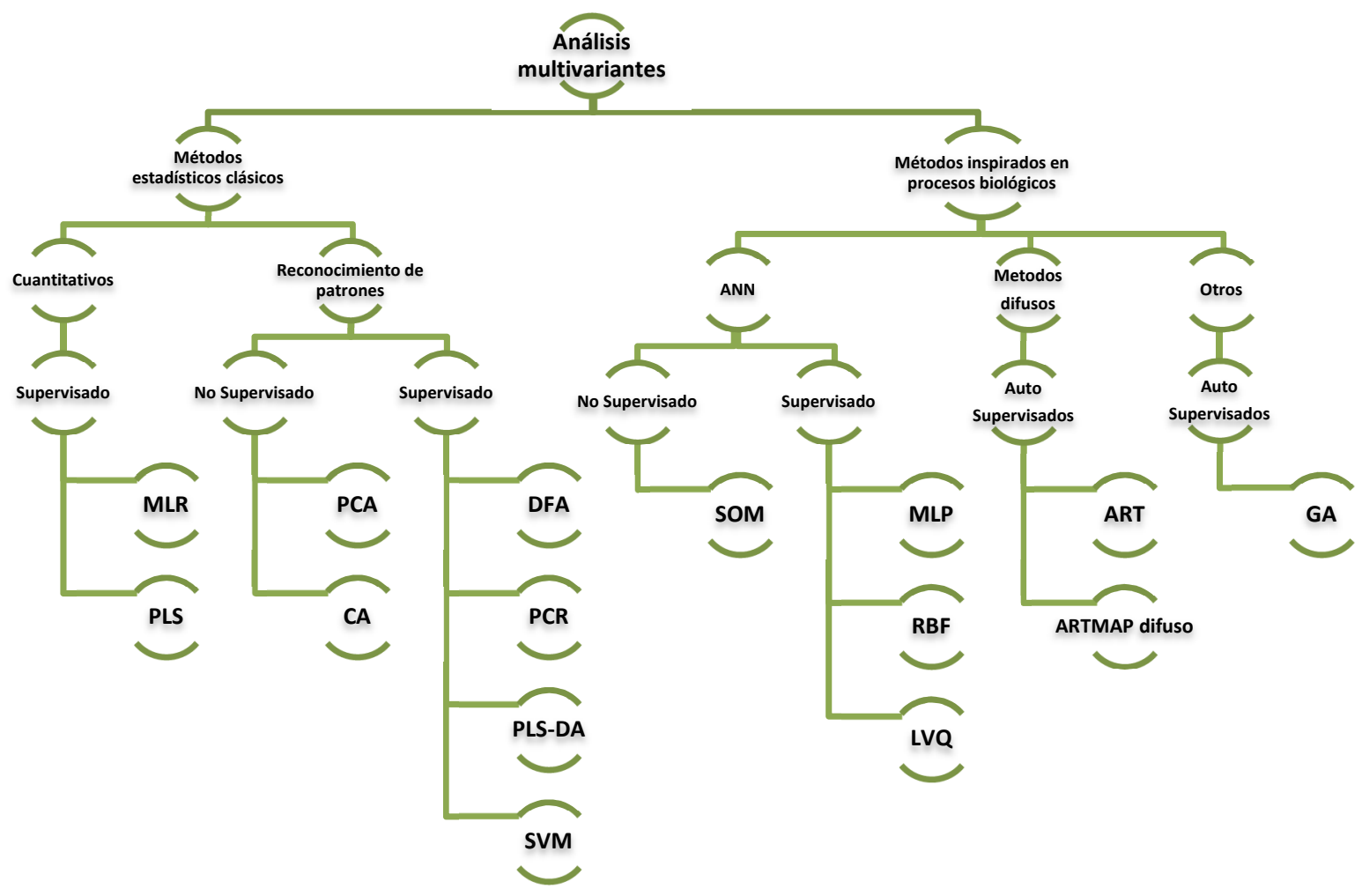

Figura 9. Resumen de clasificación de los principales métodos estadísticos utilizados en nariz electrónica. Modificado de Hines et al. (2003).

El conjunto de todos estos métodos multivariantes se pueden clasificar a su vez, en $\underline{\text { supervisados }}$ o no supervisados dependiendo si se hace o no una etapa inicial de “entrenamiento". Esta etapa de entrenamiento o calibración en los métodos supervisados, se lleva a cabo con un grupo de muestras conocidas. A partir de estas muestras se determina un modelo matemático que luego será utilizado para predecir las propiedades de las muestras problema.

Por otra parte, atendiendo a si los métodos utilizan o no como requisito de modelización algún tipo de distribución de probabilidad predeterminada estos pueden ser:

1) Paramétricos. Se basan en que los datos de los sensores pueden ser descritos por una función de densidad de probabilidad, por ejemplo, asumiendo que presentan una 
distribución normal o gaussiana con una media y varianza determinada. Estos métodos permiten identificar funciones de respuesta que facilitarán extrapolar predicciones.

2) No paramétricos. En estos casos los análisis no presuponen ningún modelo de distribución de probabilidad específico y se aplican de forma más genérica, adaptándose muy bien a la naturaleza de los datos. Estos métodos permiten modelizar de forma precisa los datos muestrales con los que se trabaja e interpolar predicciones en el espacio muestral. No obstante, no resultan muy adecuados para extrapolar predicciones a datos de muestras distintas a la utilizada para el desarrollo del modelo.

Debido al desarrollo informático en los últimos años, el número de métodos estadísticos multivariantes y algoritmos de cálculo han aumentado exponencialmente, de tal manera que el estudio particular de cada uno requeriría de un trabajo bibliográfico extenso para poder explicar los fundamentos y aplicaciones en la quimiometría u otras áreas de trabajo, como ya han realizado algunos autores (Brereton, 2007; Hastie et al., 2008). Esto excede el objetivo de la presente introducción de tesis, por lo que nos centraremos en los métodos más utilizados para el procesado de datos para la caracterización de volátiles a partir de las respuestas obtenidas por olfatometría electrónica y cromatografía de gases. Según Brezmes \& Llobet (2016), dentro de los distintos métodos estadísticos disponibles, los más utilizados en la determinación de calidad de frutas por nariz electrónica son:

\section{$\underline{\text { Análisis de componentes principales (PCA) }}$}

Actualmente el PCA es uno de los métodos multivariantes más utilizados en estudios de evaluación de volátiles (Benedetti et al., 2008; Du et al., 2010; Hernández et al., 2006; Sinesio et al., 2000; Yang et al., 2015). Esta metodología tiene un gran potencial para comprimir y extraer información de los datos, resolviendo el problema de colinealidad múltiple que otros métodos no logran (Maitra \& Yan, 2008).

Este método lineal paramétrico expresa los vectores respuesta como combinaciones lineales de todas las variables originales por medio de coeficientes ortogonales independientes. Cada nuevo vector ortogonal explica una parte de la variabilidad de los datos, disminuyendo la información contenida en cada uno a medida que aumenta su número. Éstos se pueden representar gráficamente en un par de ejes (o tres, en el caso de 
gráfico tridimensional) que representan los primeros componentes principales los cuales recogen la mayor parte de la variabilidad presente en las muestras.

Debido a su sencillez y al no ser supervisado, no es necesario utilizar dos grupos de muestras (entrenamiento y muestras problema) por lo que se pueden usar en condiciones muy diversas. Se suele utilizar mucho como método exploratorio de los resultados, sobre todo porque las representaciones de las proyecciones de los puntos en el plano que generan las primeras componentes principales facilita la interpretación de los resultados ya que la distancia entre puntos es una función lineal de la variabilidad para los caracteres en estudio (huellas electrónicas generadas por diferentes perfiles de volátiles cuando se trabaja con narices electrónicas) lo que permite apreciar adecuadamente el grado de similitud entre muestras.

\section{Análisis de función discriminante (DFA)}

Esta metodología paramétrica asume una distribución normal de los datos y busca una función discriminante que explique las agrupaciones de la variación existente. Para encontrar esta función se utiliza en primer lugar un grupo de muestras de "entrenamiento" o aprendizaje, de las que se obtiene una combinación lineal de las respuestas de los sensores multiplicadas por coeficientes de regresión lineal. Una vez obtenidos estos coeficientes, se puede usar la función para determinar a qué grupo pertenecen las muestras problema obteniendo los valores experimentales de los parámetros en el instrumento en cuestión. Este tipo de técnica es muy utilizada en la quimiometría por nariz electrónica (Hernández et al., 2008; Maul et al., 1998; Yang et al., 2015; Young et al., 1999), debido a su sencillez y poder de separación, ya que minimiza las diferencias de los individuos dentro de cada grupo conocido y maximiza las de grupos diferentes facilitando con ello las tareas de clasificación (Demir et al., 2011; McKellar et al., 2005). Sin embargo, en algunos casos, los resultados obtenidos con esta metodología se ven distorsionados si se dan problemas de colinealidad (elevada correlación) entre variables. Por otro lado, aunque es un método adecuado para propósitos de clasificación, como método exploratorio de relaciones entre diferentes tipos de muestras no es muy adecuado ya que las representaciones gráficas de las muestras en el espacio de las funciones discriminantes no son proporcionales a la similitud o disimilitud de las huellas electrónicas. 
Métodos basados en regresiones parciales mínimo cuadráticas (PLS y PLS-DA)

La regresión parcial mínimo cuadrática (PLS) busca un modelo predictivo que relacione una (o varias) variables dependientes $(\mathrm{Y})$ con varias variables explicativas (X) (Wold, 1966; Wold et al., 2001). En este análisis se utilizan variables continuas, por ejemplo, el contenido en un volátil (Y) y la huella electrónica obtenida tras su estímulo en una batería de sensores (X). Los algoritmos de cálculo de los modelos de regresión parcial PLS tratan de encontrar un conjunto de factores latentes que, por un lado explican la máxima variabilidad presente en las variables explicativas $(\mathrm{X})$ y por otro maximizan la correlación entre la variable dependiente (Y) y estas variables explicativas (X), es decir, que maximicen la covarianza entre la variable dependiente y las independientes. Esta metodología se ha utilizado para desarrollar modelos semi-cuantitativos al relacionar una medida analítica (acidez, sólidos solubles, firmeza, GC-MS, entre otras) con las respuestas de los sensores de la nariz electrónica (Llobet et al., 2001; Sanaeifar et al., 2016; Zhang et al., 2008).

Las modelizaciones a partir de la regresión PLS también se pueden refinar incrementando su precisión por medio de técnicas de selección de variables explicativas que se utilizarán en el modelo (selección de un subconjunto de variables de la matriz X). Para realizar esta selección de variables se pueden utilizar tanto métodos jerárquicos o algoritmos genéticos (Wise et al., 2006).

Por otro lado, en los últimos años, ha habido una tendencia creciente a aplicar esta metodología con fines de clasificación discriminante (PLS-DA) ya que se puede utilizar con una matriz de variables dependientes (Y) discretas que contengan información (dicotómica) de los grupos presentes en el conjunto de muestras que se utilizan como entrenamiento supervisado del modelo (Di Natale et al., 2001; Pallottino et al., 2012).

Esta metodología estadística es muy utilizada en los casos de análisis con muchas variables y pocas muestras que presentan multicolinealidad (Hubert \& Vanden Branden, 2003). Debido a su capacidad gráfica exploratoria, proporciona clara información sobre la importancia de las variables en la clasificación y en la actualidad existen programas estadísticos que otorgan una gran versatilidad para el trabajo con PLS-DA; sin embargo algunos estudios demuestran que si se utiliza sin el suficiente conocimiento estadístico puede conllevar a errores en las clasificaciones resultando mejor el uso de técnicas lineales 
más sencillas como el LDA (Brereton \& Lloyd, 2014). Este método no es ventajoso en los siguientes casos: si los grupos presentan diferente estructura en la dispersión de los datos (varianza), se deberían utilizar métodos no lineales o multilineales; cuando existen clases (variable Y) con diferente número de individuos, el PLS-DA ubica los límites de separación incorrectamente si no se modifican algunos parámetros de cálculo que asignan el centroide de la clase, aunque cuanto más clases, más difícil es modificar estos parámetros (Brereton \& Lloyd, 2014).

Más recientemente, en el área de la bioinformática han surgido nuevas mejoras a los PLSDA, creando los sparge PLS-DA (SPLS-DA) y otros, los cuales presentan ventajas frente a los anteriores en el trabajo con datos con muchas dimensiones (varios miles o más) como por ejemplo el caso de los datos de expresión genética (Filzmoser et al., 2012; Mehmood \& Ahmed, 2016).

\section{Máquinas de vectores soporte (SVM)}

Otra alternativa a los problemas no lineales de discriminación de patrones, cada vez más utilizada en diferentes áreas de trabajo, es la metodología estadística denominada máquinas de vectores soporte. Las SVMs son un conjunto de algoritmos de aprendizaje supervisado propuestos por Cortes \& Vapnik (1995) que encuentran los límites de clasificación a partir de un número de muestras de entrenamiento denominadas vectores soporte que presentan propiedades concretas. Además, se han desarrollado variaciones a los algoritmos originales, como por ejemplo las máquinas de aprendizaje extremo (ELM) que potencian su uso en regresión y clasificación multiclases (Guang-Bin Huang et al., 2012).

Una ventaja importante de estos algoritmos, es que permiten trabajar con muestras que no presentan una distribución normal y por la forma de cálculo de los límites de clasificación, reducen el sobreajuste de los modelos (overfitting) ya que al variar las muestras no asociadas a los vectores soporte, la solución de clasificación no varía, dándole una mayor robustez al método respecto a otros que clasifican según las funciones de densidad de probabilidad asociadas a cada clase; por esta misma razón, la presencia de valores atípicos no representa un mayor problema para este método. En comparación a las ANNs, las SVMs son reproducibles, ya que si se conocen los parámetros de optimización se pueden obtener nuevamente resultados idénticos a partir de los mismos datos (Xu et al., 2006). 
Sin embargo una de las grandes desventajas que presenta es que aún hoy no cuenta con un software estadístico orientado a la representación gráfica para la interpretación clara de los resultados (Brereton \& Lloyd, 2010).

\section{$\underline{\text { Métodos inspirados en redes neuronales artificiales (ANN) }}$}

Existen muestras reales con aromas muy complejos que requieren de métodos adaptativos de reconocimiento de patrones de volátiles más sofisticados, no lineales y que permitan minimizar las variaciones por deriva y ruido de los datos.

La metodología de redes neuronales artificiales se basa en imitar el comportamiento del sistema nervioso humano al procesar los datos por medio de neuronas artificiales (nodos) conectadas entre sí. Cada neurona artificial está programada para obtener un resultado a partir de cálculos matemáticos que suman factores ("estímulos") necesarios para dejar pasar o no información hacia otra neurona de la siguiente capa; de la misma manera como lo hacen las neuronas biológicas dejando pasar el estímulo sináptico. Según Hines et al. (2003) en estas redes neuronales artificiales existen 3 tipos de neuronas: entrada (input), ocultas (hidden) y salida (output) según la posición que ocupen en la estructura de la red neuronal.

El tipo de ANNs más conocido es el método supervisado MLP que por lo general se utiliza en su modalidad de propagación hacia atrás (BP: back-propagation). En este, el número de nodos neuronales de entrada viene dado por el número de sensores y el número de nodos ocultos se determina experimentalmente, mientras que las neuronas de salida, por lo general, se corresponden con el número de volátiles analizados. Este método ha sido utilizado en datos obtenidos con olfatómetro electrónico (Brezmes et al., 2000; Llobet et al., 2001, 1999), a pesar de que requiere un gran número de muestras y muchas veces son de difícil interpretación.

Otras redes neuronales artificiales utilizadas en trabajos con olfatómetros electrónicos son los mapas auto-organizados (SOM) con los cuales se obtienen resultados comparables y más precisos que los de PCA (Sinesio et al., 2000). Este análisis multivariante es uno de los pocos no supervisados dentro de los ANNs y es el que más se asemeja al funcionamiento de la corteza olfativa. Según algunas investigaciones presentan características intrínsecas beneficiosas tales como reducir las dimensiones de los datos y, 
en principio, no estar afectados por la deriva ni el ruido transitorio (Hines et al., 1997).

Por otra parte, Llobet et al. (1999) compararon los resultados de tres tipos de métodos inspirados en procesos biológicos supervisados, MLP, ARTMAP difuso y LVQ en la discriminación de madurez de bananas por el color de la piel, obteniendo mejores resultados de clasificación con ARTMAP difuso y LVQ en comparación con MLP. También comprobaron que los análisis ARTMAP difusos eran más adaptativos que los demás, aprendiendo a medida que introducían nuevos datos sin perder el conocimiento previo.

Si bien en la actualidad los ANNs son cada vez más utilizados en quimiometría debido a las ventajas antes citadas, presentan grandes problemas en la interpretación de los resultados (Hines et al., 2003) y adolecen de falta de reproducibilidad, ya que no se consigue reproducir el mismo modelo a partir de los datos originales aunque se repita el análisis (Xu et al., 2006).

\section{Algoritmos Genéticos (GA)}

Por último, los algoritmos genéticos fueron propuestos por H. Holland y se basan en los principios de evolución natural (Boilot et al., 2003; Holland, 1992). Este análisis no es muy utilizado en el caso de los olfatómetros electrónicos, seguramente debido a que las dimensiones no son el principal problema a solucionar en este área ya que los equipos cuentan con un máximo de 30 sensores (Verma et al., 2011). Sin embargo, Gardner et al. (2005) destaca la utilidad de este análisis para la selección de variables (sensores) dando resultados óptimos con las selección proporcionada por este método. 


\section{BIBLIOGRAFÍA}

Athamneh, A., Zoecklein, B., Mallikarjunan, K., 2008. Electronic nose evaluation of Cabernet Sauvignon fruit maturity. J. Wine Res. 19, 69-80.

Aubert, C., Pitrat, M., 2006. Volatile compounds in the skin and pulp of Queen Anne's pocket melon. J. Agric. Food Chem. 54, 8177-82.

Baixauli, C., 2002. Cultivos hortícolas al aire libre: sandía, in: Cajamar Caja Rural (Ed.), Serie Agricultura. pp. 535-567.

Baixauli, C., Aguilar, J., 2002. Cultivo sin suelo de hortalizas: aspectos prácticos y experiencias. Ser. Divulg. Técnica 110.

Bauchot, A.D., Mottram, D.S., Dodson, A.T., John, P., 1998. Effect of aminocyclopropane-1-carboxylic acid oxidase antisense gene on the formation of volatile esters in cantaloupe Charentais melon (cv. Vedrandais). J. Agric. Food Chem. 46, 4787-4792.

Beaulieu, J.C., 2006a. Volatile changes in cantaloupe during growth, maturation, and in stored fresh-cuts prepared from fruit harvested at various maturities. J. Am. Soc. Hortic. Sci. 131, 127-139.

Beaulieu, J.C., 2006b. Effect of cutting and storage on acetate and nonacetate esters in convenient, ready-to-eat fresh-cut melons and apples. HortScience 41, 65-73.

Beaulieu, J.C., Grimm, C.C., 2001. Identification of volatile compounds in cantaloupe at various developmental stages using solid phase microextraction. J. Agric. Food Chem. 49, 1345-1352.

Beaulieu, J.C., Ingram, D.A., Lea, J.M., Bett-Garner, K.L., 2004. Effect of harvest maturity on the sensory characteristics of fresh-cut cantaloupe. J. Food Sci. 69, S250-S258.

Beaulieu, J.C., Lea, J.M., 2006. Characterization and semiquantitative analysis of volatiles in seedless watermelon varieties using solid-phase microextraction. J. Agric. Food Chem. 54, 7789-7793.

Beltran, J., Serrano, E., López, F.J., Peruga, A., Valcarcel, M., Rosello, S., 2006. Comparison of two quantitative GC-MS methods for analysis of tomato aroma 
based on purge-and-trap and on solid-phase microextraction. Anal. Bioanal. Chem. $385,1255-1264$.

Benady, M., Simon, J.E., Charles, D.J., Miles, G.E., 1995. Fruit ripeness determination by electronic sensing of aromatic volatiles. Trans. ASAE 38, 251-257.

Benedetti, S., Buratti, S., Spinardi, A., Mannino, S., Mignani, I., 2008. Electronic nose as a non-destructive tool to characterise peach cultivars and to monitor their ripening stage during shelf-life. Postharvest Biol. Technol. 47, 181-188.

Berna, A., Amalia, 2010. Metal oxide sensors for electronic noses and their application to food analysis. Sensors 10, 3882-3910.

Bicchi, C., Cordero, C., Liberto, E., Sgorbini, B., Rubiolo, P., 2008. Headspace sampling of the volatile fraction of vegetable matrices. J. Chromatogr. A 1184, 220-233.

Bicchi, C., Iori, C., Rubiolo, P., Sandra, P., 2002. Headspace sorptive extraction (HSSE), stir bar sorptive extraction (SBSE), and solid phase microextraction (SPME) applied to the analysis of roasted Arabica coffee and coffee brew. J. Agric. Food Chem. 50, 449-459.

Biniecka, M., Caroli, S., 2011. Analytical methods for the quantification of volatile aromatic compounds. TrAC - Trends Anal. Chem. 30, 1756-1770.

Boilot, P., Hines, E.L., Gongora, M.A., Folland, R.S., 2003. Electronic noses intercomparison, data fusion and sensor selection in discrimination of standard fruit solutions. Sensors Actuators, B Chem. 88, 80-88.

Bort, F., Egea, P., Rubio, C., 2017. Nariz anatómica [Image]. URL http://recursostic.educacion.es/ciencias/biosfera/web/profesor/galeria_imagenes/im ages/olfativas.jpg (accessed 2.2.2017).

Bouma, J., Varallyay, G., Batjes, N.H., 1998. Principal land use changes anticipated in Europe. Agric. Ecosyst. Environ. 67, 103-119.

Bower, J., Holford, P., Latché, A., Pech, J.C., 2002. Culture conditions and detachment of the fruit influence the effect of ethylene on the climacteric respiration of melon. Postharvest Biol. Technol. 26, 135-146.

Brereton, R.G., 2007. Applied chemometrics for scientists. Wiley \& Sons Ltd., Chichester, West Sussex, UK. 
Brereton, R.G., Lloyd, G.R., 2014. Partial least squares discriminant analysis: taking the magic away. J. Chemom. 28, 213-225.

Brereton, R.G., Lloyd, G.R., 2010. Support vector machines for classification and regression. Analyst 135, 230-267.

Brezmes, J., Llobet, E., 2016. Electronic noses for monitoring the quality of fruit, in: Rodríguez Méndez, M.L. (Ed.), Electronic noses and tongues in food science. Elsevier, Londres, pp. 49-58.

Brezmes, J., Llobet, E., Vilanova, X., Saiz, G., Correig, X., 2000. Fruit ripeness monitoring using an electronic nose. Sensors Actuators B Chem. 69, 223-229.

Bucheli, P., Voirol, E., Torre, R.R., Lopez, J., Rytz, A., Tanksley, S.D., Petiard, V., de la Torre, R., 1999. Definition of nonvolatile markers for flavor of tomato (Lycopersicon esculentum Mill.) as tools in selection and breeding. J Agric Food Chem 47, 659-664.

Burger, Y., Sa'Ar, U., Paris, H.S., Lewinsohn, E., Katzir, N., Tadmor, Y., Schaffer, A.A., 2006. Genetic variability for valuable fruit quality traits in Cucumis melo. Isr. J. Plant Sci. 54, 233-242.

Burinsky, D.J., 2006. Mass spectrometry, in: Ahuja, S., Jespersen, N.D. (Eds.), Modern Instrumental Analysis. Elsevier Science, New York, USA, p. 896.

Camacho, F., 2000. EL cultivo de sandía apirena injertada, bajo invernadero, en el litoral mediterráneo Español. Fund. Cajamar 316.

Camino-Sánchez, F.J., Rodríguez-Gómez, R., Zafra-Gómez, A., Santos-Fandila, A., Vílchez, J.L., 2014. Stir bar sorptive extraction: recent applications, limitations and future trends. Talanta 130, 388-399.

Campo, E., Ferreira, V., Escudero, A., Cacho, J., 2005. Prediction of the wine sensory properties related to grape variety from dynamic-headspace gas chromatography-olfactometry data. J Agric Food Chem 53, 5682-5690.

Cebolla-Cornejo, J., Valcárcel, M., Herrero-Martínez, J.M., Roselló, S., Nuez, F., 2012. High efficiency joint CZE determination of sugars and acids in vegetables and fruits. Electrophoresis 33, 2416-2423.

Chachin, K., Iwata, T., 1988. Physiological and compositional changes in "Price melon" fruit during development and ripening. Bull. Univ. Osaka Prefect. Ser. B, Agric. 
Biol. 40, 27-35.

Chavagnat, 1972. Le greffage du melon dans l'ouest de la France. PHM 123.

Chomicki, G., Renner, S.S., 2015. Watermelon origin solved with molecular phylogenetics including Linnaean material: another example of museomics. New Phytol. 205, $526-532$.

Collins, J.K., Wu, G., Perkins-Veazie, P., Spears, K., Claypool, P.L., Baker, R.A., Clevidence, B.A., 2007. Watermelon consumption increases plasma arginine concentrations in adults. Nutrition 23, 261-266.

Condurso, C., Verzera, A., Dima, G., Tripodi, G., Crinò, P., Paratore, A., Romano, D., 2012. Effects of different rootstocks on aroma volatile compounds and carotenoid content of melon fruits. Sci. Hortic. 148, 9-16.

Cortes, C., Vapnik, V., 1995. Support-vector networks. Mach. Learn. 20, 273-297.

Cottyn, B.G., Boucque, C. V., 1968. Rapid method for the gas-chromatographic determination of volatile fatty acids in rumen fluid. J. Agric. Food Chem. 16, 105107.

Davis, A.R., Perkins-Veazie, P., Sakata, Y., López-Galarza, S., Maroto, J.V., Lee, S.G., Huh, Y.C., Sun, Z., Miguel, A., King, S.R., Cohen, R., Lee, J.M., 2008. Cucurbit grafting. CRC. Crit. Rev. Plant Sci. 27, 50-74.

Demir, N., Ferraz, A.C.O., Sargent, S.A., Balaban, M.O., 2011. Classification of impacted blueberries during storage using an electronic nose. J. Sci. Food Agric. 91, 17221727.

Di Natale, C., Macagnano, A., Martinelli, E., Paolesse, R., Proietti, E., D’Amico, A., 2001. The evaluation of quality of post-harvest oranges and apples by means of an electronic nose. Sensors Actuators, B Chem. 78, 26-31.

Du, X., Bai, J., Plotto, A., Baldwin, E., Whitaker, V., Rouseff, R., 2010. Electronic nose for detecting strawberry fruit maturity, in: Proceedings of the Florida State Horticultural Society, 123, pp. 259-263.

Engewald, W., Teske, J., Efer, J., 1999. Programmed temperature vaporiser-based injection in capillary gas chromatography. J. Chromatogr. A 856, 259-278.

Fallik, E., Alkali-Tuvia, S., Horev, B., Copel, A., Rodov, V., Aharoni, Y., Ulrich, D., 
Schulz, H., 2001. Characterisation of "Galia" melon aroma by GC and mass spectrometric sensor measurements after prolonged storage. Postharvest Biol. Technol. 22, 85-91.

FAO, 2014. FAOSTAT [WWW Document]. Prod. Crop. URL http://www.fao.org/faostat/en/\#data/QC (accessed 5.1.2017).

FEPEX, 2016. Exportación/Importación Española de frutas y hortalizas [WWW Document]. Export. Prod. Comunidades Autónomas. URL http://www.fepex.es/datos-del-sector/exportacion-importacion-española-frutashortalizas (accessed 5.1.2017).

Ferreira, V., San Juan, F., Escudero, A., Culleré, L., Fernández-Zurbano, P., SaenzNavajas, M.P., Cacho, J., 2009. Modeling quality of premium Spanish red wines from gas chromatography-olfactometry data. J. Agric. Food Chem. 57, 7490-7498.

Filzmoser, P., Gschwandtner, M., Todorov, V., 2012. Review of sparse methods in regression and classification with application to chemometrics. J. Chemom. 26, 4251.

Flath, R.A., Sugisawa, H., Teranishi, R., 1981. Problems in flavor research, in: Teranishi, R.., Flath, R. A. and Sugisawa, H. (Eds.), Flavor Research; Recent Advances. Marcel Dekker Inc., New York, USA.

Flynn, N.E., Meininger, C.J., Haynes, T.E., Wu, G., 2002. The metabolic basis of arginine nutrition and pharmacotherapy. Biomed. Pharmacother. 56, 427-438.

Fonseca, J.M., Rushing, J.W., Testin, R.F., 2004. The anaerobic compensation point for fresh-cut watermelon and implications for postprocess handling. HortScience 39, $562-566$.

Gardner, J.W., Bartlett, P.N., 1999. Electronic noses. Principles and applications. Meas. Sci. Technol. 11, 1087.

Gardner, J.W., Boilot, P., Hines, E.L., 2005. Enhancing electronic nose performance by sensor selection using a new integer-based genetic algorithm approach. Sensors Actuators, B Chem. 106, 114-121.

Gardner, J.W., Persaud, K.C., 2001. Electronic noses and olfaction 2000. Meas. Sci. Technol. 12, 2019-2020.

Gross, K.C., Pharr, D.M., 1982. A potential pathway for galactose metabolism in Cucumis 
sativus L., a stachyose transporting species. Plant Physiol. 69, 117-21.

Grunert, K.G., Bech-Larsen, T., Bredahl, L., 2000. Three issues in consumer quality perception and acceptance of dairy products. Int. Dairy J. 10, 575-584.

Guang-Bin Huang, Hongming Zhou, Xiaojian Ding, Rui Zhang, 2012. Extreme learning machine for regression and multiclass classification. IEEE Trans. Syst. Man, Cybern. Part B 42, 513-529.

Guarrasi, V., Farina, V., Germanà, M.A., San Biagio, P.L., Mazzaglia, A., 2011. Fruit quality evaluation of four loquat cultivars grown in Sicily. Acta Hortic. 887, 299304.

Guilbault, G.G., Jordan, J.M., Scheide, E., 1988. Analytical uses of piezoelectric crystals: a review. C R C Crit. Rev. Anal. Chem. 19, 1-28.

Gutierrez-Osuna, R., 2002. Pattern analysis for machine olfaction: a review. Sensors Journal, IEEE 2, 189-202.

Hartmann, H.T., Kest, D.E., Davies, F.T., Geneve, R.L., 1997. Principles of grafting and budding, in: Ghosh, A.K. (Ed.), Plant propagation: principles and practices. Prentice Hall, New Jersey, USA, p. 770.

Hastie, T., Tibshirani, R., Friedman, J., 2008. The elements of statistical learning. Data mining, interference, and prediction, Second. ed. Springer, Standford, California, USA.

Hernández, A., Wang, J., Hu, G., García Pereira, A., 2008. Monitoring storage shelf life of tomato using electronic nose technique. J. Food Eng. 85, 625-631.

Hernández, A., Wang, J., Hu, G., García Pereira, A., 2006. Electronic nose technique potential monitoring mandarin maturity. Sensors Actuators B Chem. 113, 347-353.

Hiatt, M., Donnelly, J., Youngman, D., 1994. Separation and isolation of volatile organic compounds using vacuum distillation with GC/MS determination. Anal. Chem. 66, 905.

Hines, E.L., Boilot, P., Gardner, J.W., Gongora, M.A., 2003. Pattern analysis for electronic noses, in: Pearce, T.C., Schiffman, S.S., Nagle, H.T., Gardner, J.W. (Eds.), Handbook of machine olfaction. Wiley-VCH Verlag GmbH \& Co. KGaA, Weinheim, Germany, pp. 133-160. 
Hines, E.L., Gardner, J.W., Potter, C.E.R., 1997. Olfactory feature maps from an electronic nose. Meas. Control 30, 262-268.

Holland, J.H., 1992. Adaptation in natural and artificial systems: An introductory analysis with applications to biology, control, and artificial intelligence. MIT Press, London, UK.

Holmberg, M., Artursson, T., 2003. Drift compensation, standards, and calibration methods, in: Pearce, T.C., Schiffman, S.S., Nagle, H.T., Gardner, J.W. (Eds.), Handbook of Machine Olfaction. Wiley-VCH Verlag GmbH \& Co. KGaA, Weinheim, Germany, pp. 325-346.

Homatidou, V.I., Karvouni, S.S., Dourtoglou, V.G., Poulos, C.N., 1992. Determination of total volatile components of Cucumis melo L. variety Cantaloupensis. J. Agric. Food Chem. 40, 1385-1388.

Hoyos, P., 2007. Situación del injerto en horticultura en España: especies, zonas de producción de planta, portainjertos. Ind. Hortícola 199, 12-25.

Hubbard, N.L., Huber, S.C., Pharr, D.M., 1989. Sucrose phosphate synthase and acid invertase as determinants of sucrose concentration in developing muskmelon (Cucumis melo L.) fruits. Plant Physiol 91, 1527-1534.

Hubert, M., Vanden Branden, K., 2003. Robust methods for partial least squares regression. J. Chemom. 17, 537-549.

Jordán, M.J., Shaw, P.E., Goodner, K.L., 2001. Volatile components in aqueous essence and fresh fruit of Cucumis melo cv. Athena (muskmelon) by GC-MS and GC-O. J. Agric. Food Chem. 49, 5929-5933.

Kader, A.A., 2002. Quality and safely factors: definition and evaluation for fresh horticultural crops, in: Postharvest technology of horticultural Crops. pp. 279-286.

Kanavouras, A., Kiritsakis, A., Hernandez, R.J., 2005. Comparative study on volatile analysis of extra virgin olive oil by dynamic headspace and solid phase microextraction. Food Chem. 90, 69-79.

Katayama, O., Kaneko, K., 1969. The aroma components in fruits and vegetables. Part V. The volatiles of watermelon (Citrullus vulgaris). Nippon Shokuhin Kogyo GakkaiShi 16, 474-479.

Katzir, N., Portnoy, V., Benyamini, Y., Yariv, Y., Tzuri, G., Pompan-Lotan, M., Larkov, 
O., Bar, E., Ibdah, M., Azulay, Y., Ravid, U., Burger, Y., Schaffer, A.A., Tadmor, Y., Lewinsohn, E., 2006. Functional genomics of genes involved in the formation of melon aroma. Cucurbitaceae 31-38.

Kemp, T.R., 1975. Identification of some volatile compounds from Citrullus vulgaris. Phytochemistry 14, 2637-2638.

Koehler, P.E., Kays, S.J., 1991. Sweet potato flavor: quantitative and qualitative assessment of optimum sweetness. J. Food Qual. 14, 241-249.

Kohlmeier, L., Kark, J.D., Gomez-Gracia, E., Martin, B.C., Steck, S.E., Kardinaal, A.F.M., Ringstad, J., Thamm, M., Masaev, V., Riemersma, R., Martin-Moreno, J.M., Huttunen, J.K., Kok, F.J., 1997. Lycopene and myocardial infarction risk in the EURAMIC study. Am J Epidemiol 146, 618-626.

Kolayli, S., Kara, M., Tezcan, F., Erim, F.B., Sahin, H., Ulusoy, E., Aliyazicioglu, R., 2010. Comparative study of chemical and biochemical properties of different melon cultivars: standard, hybrid, and grafted melons. J. Agric. Food Chem. 58, 97649769.

Kourkoutas, D., Elmore, J.S., Mottram, D.S., 2006. Comparison of the volatile compositions and flavour properties of Cantaloupe, Galia and Honeydew muskmelons. Food Chem. 97, 95-102.

Laur, L.M., Tian, L., 2011. Provitamin A and vitamin C contents in selected Californiagrown Cantaloupe and Honeydew melons and imported melons. J. Food Compos. Anal. 24, 194-201.

Lawless, H.T., Heymann, H., 2010. Sensory evaluation of food: principles and practices, Second. ed. Springer, New York, USA.

Lebrun, M., Ducamp-Collin, M.-N., Plotto, A., Goodner, K.L., Baldwin, E., 2004. Development of electronic nose measurements for mango (Mangifera indica) homogenate and whole fruit, in: Proceedings of the Annual Meeting of the Florida State Horticultural Society, 117, pp. 421-425.

Leffingwell, J.C., Alford, E.D., Leffingwell, D., 2015. Identification of the volatile constituents of raw pumpkin (Cucurbita pepo L.) by dynamic headspace analyses. Leffingwell Reports 7, 1-14.

Lester, G.E., 2008. Antioxidant, sugar, mineral, and phytonutrient concentrations across 
edible fruit tissues of orange-fleshed Honeydew melon (Cucumis melo L.). J. Agric. Food Chem. 56, 3694-3698.

Lewinsohn, E., Sitrit, Y., Bar, E., Azulay, Y., Meir, A., Zamir, D., Tadmor, Y., 2005. Carotenoid pigmentation affects the volatile composition of tomato and watermelon fruits, as revealed by comparative genetic analyses. J. Agric. Food Chem. 53, 31423148 .

Lin, S., Zhang, X., 2016. A rapid and novel method for predicting nicotine alkaloids in tobacco through electronic nose and partial least-squares regression analysis. Anal. Methods 8, 1609-1617.

Liu, C., Zhang, H., Dai, Z., Liu, X., Liu, Y., Deng, X., Chen, F., Xu, J., 2012. Volatile chemical and carotenoid profiles in watermelons [Citrullus vulgaris (Thunb.) Schrad (Cucurbitaceae)] with different flesh colors. Food Sci. Biotechnol. 21, 531541.

Llobet, E., Hines, E.L., Gardner, J.W., Franco, S., 1999. Non-destructive banana ripeness determination using a neural network-based electronic nose. Meas. Sci. Technol. $10,538-548$.

Llobet, E., Ionescu, R., Al-Khalifa, S., Brezmes, J., Vilanova, X., Correig, X., Bârsan, N., Gardner, J.W., 2001. Multicomponent gas mixture analysis using a single tin oxide sensor and dynamic pattern recognition. IEEE Sensors J. 13, 207-213.

López, A.F., 2003. Manual para la preparación y venta de frutas y hortalizas del campo al mercado. [WWW Document]. Boletín Serv. agrícolas la FAO 151. URL http://www.fao.org/docrep/006/Y4893S/Y4893S00.HTM (accessed 4.12.2017).

Maitra, S., Yan, J., 2008. Principle component analysis and partial least squares: two dimension reduction techniques for regression. Casualty Actuar. Soc. 79-90.

Mandel, H., Levy, N., Izkovitch, S., Korman, S.H., 2005. Elevated plasma citrulline and arginine due to consumption of Citrullus vulgaris (watermelon). J. Inherit. Metab. Dis. 28, 467-472.

MAPAMA, 2015. Anuario de estadística - Publicaciones - Estadísticas [WWW Document]. Superf. y Prod. Cultiv. Hortalizas fruto-Sandía Análisis Prov. Superf. Rend. $\quad$ y $\quad$ Prod. $2014 . \quad$ URL http://www.mapama.gob.es/es/estadistica/temas/publicaciones/anuario-de- 
estadistica/2015/default.aspx ?parte $=3 \&$ capitulo $=13 \&$ grupo $=6 \&$ seccion $=20$ (accessed 5.2.2017).

Maroto, J. V., 2002. Botánica (taxonomía y fisiología) y adaptabilidad de la sandía, in: Mundi-Prensa (Ed.), El cultivo de la sandía. Fundación Caja Rural Valencia, Madrid, Spain, pp. 29-42.

Marrazzo, W.N., Heinemann, P.H., Crassweller, R.E., Leblanc, E., 2005. Electronic nose chemical sensor feasibility study for the differentiation of apple cultivars. 48, 19952002

Martí, R., Roselló, S., Cebolla-Cornejo, J., 2016. Tomato as a source of carotenoids and polyphenols targeted to cancer prevention. Cancers (Basel). 8, 58.

Martin, A.J.P., Synge, R.L.M., 1941. A new form of chromatogram employing two liquid phases. 1. A theory of chromatography. 2. Application to the micro-determination of the higher monoamino-acids in proteins. Chromatogr. Amin. Acids 151, 13581368.

Maul, F., Sargent, S.A., Balaban, M.O., Baldwin, E.A., Huber, D.J., Sims, C.A., 1998. Aroma volatile profiles from ripe tomatoes are influenced by physiological maturity at harvest: an application for electronic nose technology. J. Am. Soc. Hortic. Sci. 123, 1094-1101.

McKellar, R.C., Rupasinghe, H.P.V., Lu, X., Knight, K.P., 2005. The electronic nose as a tool for the classification of fruit and grape wines from different Ontario wineries. J. Sci. Food Agric. 85, 2391-2396.

Mehmood, T., Ahmed, B., 2016. The diversity in the applications of partial least squares: an overview. J. Chemom. 30, 4-17.

Miguel, A., 2009. Evolución del injerto de hortalizas en España. Hortic. Int. 10, 72.

Miguel, A., Maroto, J. V., San Bautista, A., Baixauli, C., Cebolla, V., Pascual, B., López, S., Guardiola, J.L., 2004. The grafting of triploid watermelon is an advantageous alternative to soil fumigation by methyl bromide for control of Fusarium wilt. Sci. Hortic. 103, 9-17.

Monforte, A.J., Alvarez, J.M., 2006. Mejora de la calidad del melón, in: Llácer, G., Díez, M.J., Carrillo, J.M., Badenes, M.L. (Eds.), Mejora genética de la calidad en plantas. Soc. Española de Ciencias Hortícolas; Soc. Española de Genética, Valencia, Spain, 
pp. 393-413.

Monforte, A.J., Diaz, A., Caño-Delgado, A., Van Der Knaap, E., 2014. The genetic basis of fruit morphology in horticultural crops: lessons from tomato and melon. J. Exp. Bot. 65, 4625-4637.

Morales, M.T., Aparicio, R., Gutiérrez, F., 1992. Técnicas de aislamiento y concentración de volátiles de aceites vegetales. Grasas y Aceites 43, 164-173.

Moreiras, O., Carbajak, Á., Cabrera, L., Cuadrado, C., 2013. Tablas de composición de alimentos, 16th ed. Pirámide, Madrid, Spain.

Moreno, I., Caballero, R., Galán, R., Matía, F., Jiménez, A., 2009. La nariz electrónica: estado del arte. Rev. Iberoam. Automática e Informática Ind. (RIAI) 6, 76-91.

Muramatsu, Y., 1981. Problems on vegetable grafting (In Japanese). Shisetu Engei 10,11, $48-53,46-52$.

Nanto, H., Stetter, J.R., 2003. Introduction to chemosensors, in: Pearce, T.C., Schiffman, S.S., Nagle, H.T., Gardner, J.W. (Eds.), Handbook of machine olfaction. WileyVCH Verlag GmbH \& Co. KGaA, Weinheim, Germany, pp. 79-104.

Obando-Ulloa, J.M., Moreno, E., García-Mas, J., Nicolai, B., Lammertyn, J., Monforte, A.J., Fernández-Trujillo, J.P., 2008. Climacteric or non-climacteric behavior in melon fruit: 1. Aroma volatiles. Postharvest Biol. Technol. 49, 27-37.

Obando-Ulloa, J.M., Ruiz, J., 2010. Aroma profile of a collection of near-isogenic lines of melon (Cucumis melo L.). Food Chem. 118, 815-822.

Pallottino, F., Costa, C., Antonucci, F., Strano, M.C., Calandra, M., Solaini, S., Menesatti, P., 2012. Electronic nose application for determination of Penicillium digitatum in Valencia oranges. J. Sci. Food Agric. 92, 2008-2012.

Pardo, J.E., Gómez, R., Tardáguila, J., Amo, M., Varón, R., 1997. Quality evaluation of watermelon varieties. J. Food Qual. 20, 547-557.

Pawliszyn, J., 2000. Theory of solid-phase microextraction. J. Chromatogr. Sci. 38, 270278.

Pawliszyn, J., 1997. Solid phase microextraction: theory and practice. Wiley-VCH, Waterloo, Ontario, Canada.

Pérez, E., 2015. Cultivo de sandía en suelo e hidroponía, in: Gázquez Garrido, J.C. (Ed.), 
Técnicas de cultivo y comercialización de la sandía. pp. 17-72.

Perry, P.L., Wang, Y., Lin, J., 2009. Analysis of Honeydew melon (Cucumis melo var. inodorus) flavour and GC-MS/MS identification of (E,Z)-2,6-nonadienyl acetate. Flavour Fragr. J. 24, 341-347.

Persaud, K., Dodd, G., 1982. Analysis of discrimination mechanisms in the mammalian olfactory system using a model nose. Nature 299, 352-5.

Petropoulos, S.A., Olympios, C., Ropokis, A., Vlachou, G., Ntatsi, G., Paraskevopoulos, A., Passam, H.C., 2014. Fruit volatiles, quality, and yield of watermelon as affected by grafting. J. Agric. Sci. Technol. 16, 873-885.

Pino, J.A., Marbot, R., Aguero, J., 2003. Volatile components of watermelon (Citrullus lanatus [Thunb.] Matsum. et Nakai) fruit. J. Essent. Oil Res. 15, 379-380.

Pitrat, M., Chauvet, M., Foury, C., 1999. Diversity, history and production of cultivated cucurbits. Acta Hortic. 492, 21-28.

Pitrat, M., Hanelt, P., Hammer, K., 2000. Some comments on infraspecific classification of cultivars of melon. Acta Hortic. 510, 29-36.

Pokhum, C., Chawengkijwanich, C., Maolanon, R., 2010. Application of electronic-nose for identification of ripeness stage of durian. Acta Hort. 875, 319-328.

Pretel, M.T., Serrano, M., Riquelme, F., Romojaro, F., 1993. La calidad de la producción hortícola, in: Albi, M.A., Gutiérrez, F., Roca, M. (Eds.), La calidad en frutos y hortalizas. Maduración y post-recolección’ 93, Sevilla, Spain.

RAE, 2014. Diccionario Usual [WWW Document]. Edición del Tricentenario. URL http://dle.rae.es/srv/fetch?id=6nVpk8P (accessed 6.28.2017).

Reche, J., 2008. Cultivo del melón en invernadero. Junta Andalucía. Cons. Agric. y Pesca. 307.

Reddy, U.K., Nimmakayala, P., Levi, A., Abburi, V.L., Saminathan, T., Tomason, Y.R., Vajja, G., Reddy, R., Abburi, L., Wehner, T.C., Ronin, Y., Karol, A., 2014. Highresolution genetic map for understanding the effect of genome-wide recombination rate on nucleotide diversity in watermelon. Genes|Genomes|Genetics 4, 2219-2230.

Rimando, A.M., Perkins-Veazie, P.M., 2005. Determination of citrulline in watermelon rind. J. Chromatogr. A 1078, 196-200. 
Rouphael, Y., Schwarz, D., Krumbein, A., Colla, G., 2010. Impact of grafting on product quality of fruit vegetables. Sci. Hortic. 127, 172-179.

Ruberto, G., Renda, A., Amico, V., Tringali, C., 2008. Volatile components of grape pomaces from different cultivars of Sicilian Vitis vinifera L. Bioresour. Technol. 99, 260-268.

Saftner, R., Abbott, J.A., Lester, G., Vinyard, B., 2006. Sensory and analytical comparison of orange-fleshed Honeydew to Cantaloupe and green-fleshed Honeydew for freshcut chunks. Postharvest Biol. Technol. 42, 150-160.

Sales, C., Cervera, M.I., Gil, R., Portolés, T., Pitarch, E., Beltran, J., 2017. Quality classification of Spanish olive oils by untargeted gas chromatography coupled to hybrid quadrupole-time of flight mass spectrometry with atmospheric pressure chemical ionization and metabolomics-based statistical approach. Food Chem. 216, 365-373.

Sanaeifar, A., Mohtasebi, S.S., Ghasemi-Varnamkhasti, M., Ahmadi, H., 2016. Application of MOS based electronic nose for the prediction of banana quality properties. Meas. J. Int. Meas. Confed. 82, 105-114.

Schaller, E., Bosset, J.O., Escher, F., 1998. "Electronic noses" and their application to food. Leb. und Technologie 31, 305-316.

Shalit, M., Katzir, N., Tadmor, Y., Larkov, O., Burger, Y., Shalekhet, F., Lastochkin, E., Ravid, U., Amar, O., Edelstein, M., Karchi, Z., Lewinsohn, E., 2001. Acetyl-CoA: alcohol acetyltransferase activity and aroma formation in ripening melon fruits. J. Agric. Food Chem. 49, 794-799.

Sides, A., Robards, K., Helliwell, S., 2000. Developments in extraction techniques and their application to analysis of volatiles in foods. TrAC - Trends Anal. Chem. 19, 322-329.

Simon, J.E., Hetzroni, A., Bordelon, B., Miles, G.E., Charles, D.J., 1996. Electronic sensing of aromatic volatiles for quality sorting of blueberries. J. Food Sci. 61, 967-970.

Sinesio, F., Di Natale, C., Quaglia, G.B., Bucarelli, F.M., Moneta, E., Macagnano, A., Paolesse, R., D'Amico, A., 2000. Use of electronic nose and trained sensory panel in the evaluation of tomato quality. J. Sci. Food Agric. 80, 63-71. 
Snow, N.H., Slack, G.C., 2002. Head-space analysis in modern gas chromatography. TrAC Trends Anal. Chem. 21, 608-617.

Solis-Solis, H.M.M., Calderon-Santoyo, M., Gutierrez-Martinez, P., Schorr-Galindo, S., Ragazzo-Sanchez, J.A.A., 2007. Discrimination of eight varieties of apricot (Prunus armeniaca) by electronic nose, LLE and SPME using GC-MS and multivariate analysis. Sensors Actuators B 125, 415-421.

Stashenko, E.E., Martínez, J.R., 2007. Sampling volatile compounds from natural products with headspace/solid-phase micro-extraction. J. Biochem. Biophys. Methods 70, $235-242$.

Stepansky, A., Kovalski, I., Perl-Treves, R., 1999a. Intraspecific classification of melons (Cucumis melo L.) in view of their phenotypic and molecular variation. Plant Syst. Evol. 217, 313-333.

Stepansky, A., Kovalski, I., Schaffer, A.A., Perl-Treves, R., 1999b. Variation in sugar levels and invertase activity in mature fruit representing a broad spectrum of Cucumis melo genotypes. Genet. Resour. Crop Evol. 46, 53-62.

Suzuki, E., 1972. Sandía de Yamato. Folleto Informativo.

Swinnterton, J.W., Linnenbom, V.J., Cheek, C.H., 1962. Determination of dissolved gases in aqueous solutions by gas chromatography. Anal. Chem. 34, 483-485.

The Davis Enterprise, 2014. Sandías variedades [Image]. URL http://www.davisenterprise.com/media-post/watermelonphotos/attachment/watermellon2w/ (accessed 2.2.2017).

Tieman, D., Zhu, G., Resende, M.F.R., Lin, T., Nguyen, C., Bies, D., Rambla, J.L., Ortiz, K.S., Taylor, M., Zhang, B., Ikeda, H., Liu, Z., Fisher, J., Zemach, I., Monforte, A., Zamir, D., Granell, A., Kirst, M., Huang, S., Klee, H., 2017. A chemical genetic roadmap to improved tomato flavor. Plant Sci. 355, 391-394.

Tienpont, B., David, F., Bicchi, C., Sandra, P., 2000. High capacity headspace sorptive extraction. J. Microcolumn Sep. 12, 577-584.

Tolón, A., Lastra, X., 2010. La agricultura intensiva del poniente almeriense. Diagnóstico e instrumentos de gestión ambiental. M+A. Rev. Electrónica Medioambiente 8, 1-22.

Torres, J.M., Miquel, M.J., 2003. La geografía del comercio del melón. Hortic. Int. 16-25. 
Ueda, Y., Fujishita, N., Chachin, K., 1997. Presence of alcohol acetyltransferase in melons (Cucumis melo L.). Postharvest Biol. Technol. 10, 121-126.

Valcárcel, M., 2009. Optimización del proceso de evaluación y seleción de germoplasma de tomate por características de calidad organoléptica: uso de la tecnología NIR y sensores electrónicos. PhD. Thesis. Escola Superior de Tecnologia i Ciències Experimentals - Departament de Ciències Agràries i del Medi Natural, Universitat Jaume I, Castellón de la Plana, Spain.

Van Duyn, M.A.S., Pivonka, E., 2000. Overview of the health benefits of fruit and vegetable consumption for the dietetics professional. J. Am. Diet. Assoc.

Verma, P., Somvanshi, D., Yadava, R.D.S., 2011. On genetic algorithm and multiple preprocessors assisted feature boosting for electronic nose signal processing. Int. J. Comput. Intell. Res. 7, 271-294.

Verzera, A., Dima, G., Tripodi, G., Condurso, C., Crinò, P., Romano, D., Mazzaglia, A., Lanza, C.M., Restuccia, C., Paratore, A., 2014. Aroma and sensory quality of Honeydew melon fruits (Cucumis melo L. subsp. melo var. inodorus H. Jacq.) in relation to different rootstocks. Sci. Hortic. 169, 118-124.

Verzera, A., Dima, G., Tripodi, G., Ziino, M., 2011. Fast quantitative determination of aroma volatile constituents in melon fruits by headspace-solid-phase microextraction and gas chromatography-mass spectrometry. Food Anal. Methods $4,141-149$.

Villanueva, M.J., Tenorio, M.D., Esteban, M.A., Mendoza, M.C., 2004. Compositional changes during ripening of two cultivars of muskmelon fruits. Food Chem. 87, $179-185$.

Villén, J., Señoráns, F.J., Reglero, G., Herraiz, M., Villen, J., Seiiorans, F.J., 1995. Analysis of wine aroma by direct injection in gas chromatography without previous extraction. J. Agric. Food Chem. 43, 717-722.

Wang, Y.M., Wyllie, S.G., Leach, D.N., 1996. Chemical changes during the development and ripening of the fruit of Cucumis melo (cv. Makdimon). J. Agric. Food Chem. 44, 210-216.

Wasylikowa, K., Van Der Veen, M., 2004. An archaeobotanical contribution to the history of watermelon, Citrullus lanatus (Thunb.) Matsum. \& Nakai (syn. C. vulgaris 
Schrad.). Veg. Hist. Archaeobot. 13, 213-217.

Weidong, C., Hongwen, C., Bingkui, Z., n.d. Analysis of sugar content of watermelon (Citrullus lanatus ( Thunb .) Mansf .). Cucurbit Genet. Coop. Rep. 25, 30-31.

Wise, B.M., Gallagher, N.B., Bro, R., Shaver, J.M., Windig, W., Koch, R.S., 2006. Chemometrics Tutorial for PLS_Toolbox and Solo, MATLABTM, 4th ed, Eigenvector Research, Inc.

Wold, H., 1966. Estimation of principal components and related models by iterative least squares, in: Multivariate analysis. Academic Press, New York, USA, pp. 391-420.

Wold, S., Sjöström, M., Eriksson, L., 2001. PLS-regression: a basic tool of chemometrics. Chemom. Intell. Lab. Syst. 58, 109-130.

Xu, Y., Zomer, S., Brereton, R.G., 2006. Support vector machines: a recent method for classification in chemometrics. Crit. Rev. Anal. Chem. 36, 177-188.

Yajima, I., Sakakibara, H., Ide, J., Yanai, T., Hayashi, K., 1985. Volatile flavor components of watermelon (Citrullus vulgaris). Agric. Biol. Chem. 49, 3145-3150.

Yamaguchi, M., Hughes, D.L., Yabumoto, K., Jennings, W.G., 1977. Quality of cantaloupe muskmelons: variability and attributes. Sci. Hortic. 6, 59-70.

Yang, S.L., Xie, S.P., Xu, M., Zhang, C., Wu, N., Yang, J., Zhang, L., Zhang, D.Y., Jiang, Y., Wu, C.J., 2015. A novel method for rapid discrimination of bulbus of Fritillaria by using electronic nose and electronic tongue technology. Anal. Methods 7, 943952.

Yariv, Y., Portnoy, V., Burger, Y., Benyamini, Y., Lewinsohn, E., Tadmor, Y., Ravid, U., White, R., Giovannoni, J., Schaffer, A.A., Katzir, N., 2004. Isolation and characterization of fruit-related genes in melons (Cucumis melo) using SSH and macroarray techniques, in: Lebeda, A., Paris, H.S. (Eds.), Progress in cucurbit genetics and breeding research. Olomouc, Czech Republic, pp. 491-497.

Yativ, M., Harary, I., Wolf, S., 2010. Sucrose accumulation in watermelon fruits: genetic variation and biochemical analysis. J. Plant Physiol. 167, 589-596.

Yoo, K.S., Bang, H., Lee, E.J., Crosby, K., Patil, B.S., 2012. Variation of carotenoid, sugar, and ascorbic acid concentrations in watermelon genotypes and genetic analysis. Hortic. Environ. Biotechnol. 53, 552-560. 
Young, H., Rossiter, K., Wang, M., Miller, M., 1999. Characterization of Royal Gala apple aroma using electronic nose technology-potential maturity indicator. J. Agric. Food Chem. 47, 5173-5177.

Zawirska-Wojtasiak, R., Gośliński, M., Szwacka, M., Gajc-Wolska, J., Mildner-Szkudlarz, S., 2009. Aroma evaluation of transgenic, thaumatin II-producing cucumber fruits. J. Food Sci. 74, 204-210.

Zhang, H., Wang, J., Ye, S., 2008. Predictions of acidity, soluble solids and firmness of pear using electronic nose technique. J. Food Eng. 86, 370-378. 


\section{OBJETIVOS}



En un tiempo en el que la calidad organoléptica es cada vez más valorada, es preciso contar con herramientas que nos permitan fenotiparla de forma clara y sencilla. Esto es especialmente importante en programas de mejora en los que se debe cribar gran cantidad de material en poco tiempo. Entre los componentes de la calidad organoléptica interna se ha prestado mucha atención a aquellos relacionados con la percepción del gusto, inicialmente con técnicas sencillas como la determinación de sólidos solubles totales por refractometría y actualmente con métodos más precisos como la cromatografía líquida de alta resolución o la electroforesis capilar. Sin embargo, al aroma, por su complejidad y coste de análisis se le ha prestado menor atención. Hoy en día se sabe que ha sido un error, puesto que se ha demostrado en especies como el tomate que los volátiles pueden potenciar la percepción del gusto y la falta de atención a estos compuestos en programas de mejora se ha manifestado como una de las causas de pérdida de calidad en cultivares modernos.

En este contexto, la presente tesis pretende contribuir al conocimiento del perfil volátil relacionado con el aroma de dos prominentes cucurbitáceas, el melón y la sandía a través del desarrollo de técnicas de fenotipado y su aplicación a la evaluación de portainjertos experimentales en programas de mejora. Este objetivo se desarrolla en los siguientes objetivos parciales:

- Desarrollo de un método de análisis de aromas aplicable a melón y sandía basado en extracción mediante purga y trampa con cartuchos de fase sólida (SPE) que permita cuantificar de forma fiable los principales volátiles de estas cucurbitáceas.

- Evaluación del efecto de portainjertos experimentales sobre la calidad organoléptica de la sandía, con especial énfasis en los cambios producidos en el perfil volátil.

- Desarrollo de metodología de fenotipado masivo basada en olfatómetro electrónico que permita cribar material en función de su perfil volátil. 



\section{CAPÍTULO 1}

"Quantification of prominent volatile compounds responsible for muskmelon and watermelon aroma by purge and trap extraction followed by gas chromatography-mass spectrometry determination"

Fredes A., Sales C., Barreda M., Valcárcel M., Roselló S., Beltrán J. (2016). Food Chemistry, 190, 689-700.

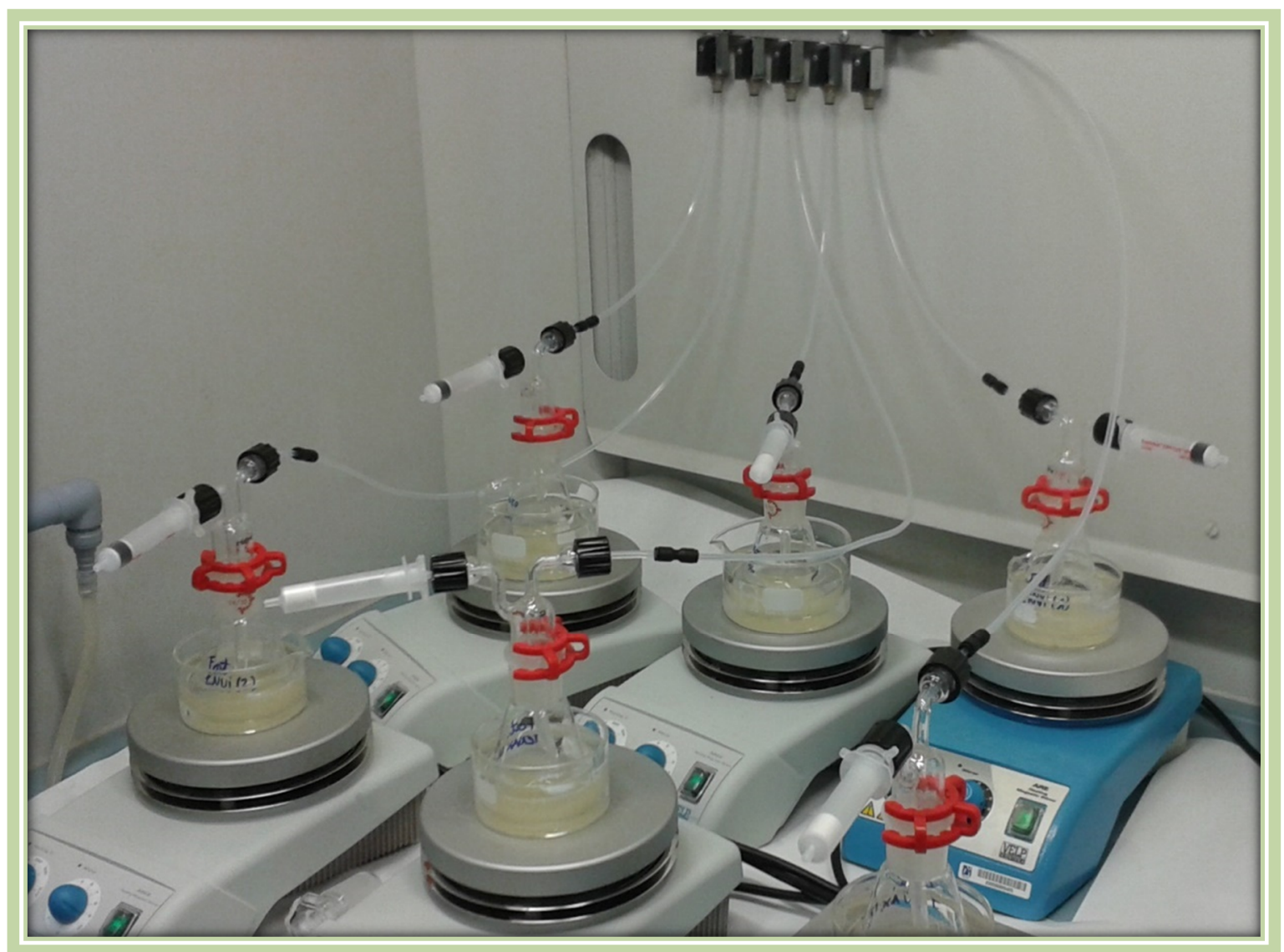



Analytical Methods

Quantification of prominent volatile compounds responsible for muskmelon and watermelon aroma by purge and trap extraction followed by gas chromatography-mass spectrometry determination

Alejandro Fredes ${ }^{\mathrm{a}}$, Carlos Sales ${ }^{\mathrm{b}}$, Mercedes Barreda ${ }^{\mathrm{b}}$, Mercedes Valcárcel ${ }^{\mathrm{a}}$, Salvador Roselló ${ }^{\mathrm{a}}$, Joaquim Beltrán ${ }^{\mathrm{b}, *}$

Department de Ciències Agràries i del Medi Natural, Universitat Jaume I, Avda. Sos Baynat s/n, E-12071 Castellón, Spain Institut Universitari de Plaguicides i Aigües, Universitat Jaume I, Avda. Sos Baynat s/n, E-12071 Castellón, Spain

\section{ABSTRACT}

A dynamic headspace purge-and-trap (DHS-P\&T) methodology for the determination and quantification of 61 volatile compounds responsible for muskmelon and watermelon aroma has been developed and validated. The methodology is based on the application of purgeand-trap extraction followed by gas chromatography coupled to (ion trap) mass spectrometry detection. For this purpose two different P\&T sorbent cartridges have been evaluated. The influence of different extraction factors (sample weight, extraction time, and purge flow) on extraction efficiency has been studied and optimised using response surface methodology. Precision, expressed as repeatability, has been evaluated by analysing six replicates of real samples, showing relative standard deviations between $3 \%$ and $27 \%$. Linearity has been studied in the range of $10-6130 \mathrm{ng} \mathrm{mL}^{-1}$ depending on the compound response, showing coefficients of correlation between 0.995 and 0.999 . Detection limits ranged between 0.1 and $274 \mathrm{ng} \mathrm{g}^{-1}$. The methodology developed is well suited for analysis of large numbers of muskmelon and watermelon samples in plant breeding programs.

Keywords: GC-MS, P\&T, Organoleptic quality, Cucurbitaceae, Response surface methodology. 


\section{INTRODUCTION}

In recent years, there has been increasing concern among consumers regarding fruit and vegetable organoleptic quality. In developed countries, an appreciable percentage of consumers are willing to pay a premium for high organoleptic quality products, mainly for fruits and vegetables. Considering that muskmelons and watermelons are important horticultural crops around the world with an increasing demand, the determination of the organoleptic quality characteristics is becoming highly important. These determinations are relevant as quality controls in the production/storage/distribution system and in research programmes, especially in the breeding sector in which obtaining new cultivars with better flavour characteristics is an increasingly important objective.

Muskmelon and watermelon flavour is directly related to their sugar content, mainly sucrose, which can reach $97 \%$ of the total soluble solids content (Kultur et al., 2001; Yau et al., 2010). Additionally, other minor components, such as organic acids and especially volatile compounds, also contribute to define flavour nuances, and they can also modulate the perception of sweetness (Monforte \& Alvarez, 2006; Yamaguchi et al., 1977). This influence of aroma on the overall flavour perception adds credence to the recommendation that aroma should be evaluated along with the soluble solids content. It has been observed that high levels of soluble solids do not always have correspondingly high organoleptic quality values (Lester et al., 1992; Yabumoto et al., 1978). Moreover, the high variability of the aromatic characteristics of muskmelon (Beaulieu, 2006a; Horvat \& Senter, 1987; Wyllie et al., 1995) and watermelon (Kim et al., 1999; Yajima et al., 1985) makes this an attribute to be considered as a quality trait with increasing importance.

Evaluation of organoleptic quality is difficult. A commonly used determination of this complex quality attribute of fruits and vegetables is the evaluation of sensory characteristics by a taste panel. Nevertheless, evaluations of sensory attributes are often not readily interpreted and the number of samples that can be evaluated in a single session is low. Olfactometry combined with triangle tests would be a good approach to simplify these evaluations and uses a more speedy method but, despite its simple data interpretation, is rather tedious and needs huge sample numbers. In this context, there has been great interest in the substitution of these tedious and expensive evaluations by an objective quantification of compounds that correlates with the sensory evaluation. 
A high number of compounds with different degrees of volatility including esters, alcohols, and carbonyl compounds (especially those containing a nine-carbon straight chain) are the major chemical determinants of muskmelon fruit quality as perceived by consumers (Beaulieu \& Lancaster, 2007; Gonda et al., 2010). Until now, around 300 volatile compounds have been identified in muskmelon and watermelon samples (Aubert \& Pitrat, 2006; Beaulieu, 2006a, 2006b; Beaulieu \& Grimm, 2001; Beaulieu \& Lancaster, 2007; Beaulieu \& Lea, 2006; Homatidou et al., 1992; Jordán et al., 2001; Kourkoutas et al., 2006; Liu et al., 2012; Obando-Ulloa et al., 2008; Obando-Ulloa \& Ruiz, 2010; Perry et al., 2009; Saftner et al., 2006; Verzera et al., 2011). Due to the important role of volatile components in organoleptic quality of foods and beverages, these compounds have been widely studied and analytical methodologies for their determination in fruit matrices are continuously being developed, including both GC (Beaulieu et al., 2004; Beaulieu \& Lancaster, 2007; Beaulieu \& Lea, 2006; Gonda et al., 2010; Kemp, 1975; Kourkoutas et al., 2006; Liu et al., 2012) and GC-O (Jordán et al., 2001; Lignou et al., 2014) methods.

A direct headspace injection could be a simple, cheap and fast option for the analysis of aromatic volatile compounds in vegetal samples. However, many volatile analytes in the vapour phase of this kind of sample are often present in too low concentrations (Beltran et al., 2006). Beaulieu \& Grimm (2001) and Beaulieu (2006b) extensively analysed melon aroma using solid-phase microextraction (SPME), reporting and verifying 25 of the aforementioned compounds for the first time in cantaloupe melons. Since then, several studies reported SPME as a good semi-quantitative methodology for the determination of volatile organic compounds (VOCs) in several vegetal samples including muskmelon (Kourkoutas et al., 2006; Verzera et al., 2011). This simple methodology presents some advantages such as a good compound pre-concentration in vapour and liquid phases, a solventless sample preparation method, short extraction times, and the possibility of using different sorbent materials depending on the nature of target analytes. However, this methology presents some disadvantages in the quantification process: the amount of analytes transferred from a sample to the SPME fibre is highly dependent on the sample matrix, adsorption capability, and several other extraction-related parameters (Bicchi et al., 2004).

On the other hand, headspace purge-and-trap technique dynamically captures the volatile sample fraction in a solid adsorbent, which can be recovered by subsequent thermal or solvent desorption. According to Contarini \& Povolo (2002) the purge-and-trap technique 
can be more suited to the determination of compounds with lower molecular weight than SPME. The main advantages of the former are the possibility to extract several samples at the same time together with the use of calibration curves in solvent for quantification. In SPME, calibration has to be performed in spiked sample matrices, as obtaining blank samples to prepare calibration curves is a problem in some cases. In addition, although it is well known that a SPME fibre can be repeatedly used (more than 100 times in automated devices) calibration curves should be done now and then to ensure quantitation, especially for very complex samples. Therefore, the P\&T technique allows quantitation of the volatiles of interest, which is an advantage compared to traditional qualitative SPME methods. Likewise, when a large number of samples needs to be analysed, the P\&T methodology has two important advantages that will facilitate its use in plant breeding program contexts. Firstly, the relatively low cost and amount of solvents involved and, secondly, the capability of extracting several samples simultaneously (depending on the laboratory configuration). Consequently the aim of the present study has been the development and validation of a dynamic headspace purge and trap methodology for volatile compounds extraction followed by gas chromatography coupled to mass spectrometry (GC-MS) determination (Buttery et al., 1982; Homatidou et al., 1992; Kemp, 1975; Verzera et al., 2011) that might be effectively applied to the analysis of a large number of muskmelon and watermelon samples.

\section{MATERIAL AND METHODS}

\subsection{Target compounds selected}

An extensive literature review showed that around 300 volatile organic compounds have been described to be present in muskmelon and watermelon samples (Supplementary information, Table S1). However, there are considerable differences in concentration, effect on muskmelon-like or watermelon-like aroma, or feasibility to be detected by humans due to their odour thresholds. In most of the reviewed studies, mainly in those where the authors compared muskmelon varieties and maturity stages, the number of relevant compounds was around or below ninety (Amaro et al., 2012; Beaulieu \& Grimm, 2001; Obando-Ulloa et al., 2008; Verzera et al., 2011). 
The compounds studied in this work were selected following two criteria: the number of papers in which they were cited and the concentration levels reported. In addition, chromatographic behaviour of the selected compounds in the GC-MS system used was also considered. Table 1 shows the 61 volatile compounds analysed in the present work.

\subsection{Samples}

Cultivars of five muskmelon types and one of watermelon commonly produced in Spain were cultivated in the open air in a spring-summer cycle at Jaume I University with common growing conditions. The plant materials were selected according to their economic importance in the Mediterranean basin and in order to study different aromatic fruit profiles.

Fruits from "Piel de sapo" muskmelon type (Cucumis melo L. var saccharinus Naud.), the most economically important type in Spain, were used in the optimisation of the volatile organic compounds (VOCs) extraction. "Charentais" muskmelon type (Cucumis melo var. cantalupensis Naud.) was used in the validation method because it has an aromatic profile with the highest number of volatile compounds.

Fruits of "Tendral" type (Cucumis melo L. var inodorus Naud.), "Charentais" type (Cucumis melo L. var cantalupensis Naud.), "Galia', type (Cucumis melo L. var reticulatus Ser.), "Amarillo" and "Piel de sapo" types (Cucumis melo L. var saccharinus Naud.) and watermelon (Citrullus vulgaris Schrad) were used for the real sample quantitation analysis. Fruits were harvested at commercially optimum maturity states by checking several morphological attributes and basic organoleptic properties, such as soluble solid content (minimum 12 'Brix for "Charentais" and "Galia" types and minimum $10^{\circ}$ Brix for the other types). After sampling, fruits were ground in a Thermomix TM31 blender (Vorwerk España M.S.L., Madrid, Spain) in the case of the "Piel de Sapo" used for optimisation of the method for enough time to obtain a homogenous sample; the other melon types were processed in an $1100 \mathrm{~W}$ blender until they were completely homogenised. In both cases samples were immediately stored at $80^{\circ} \mathrm{C}$ until analysis. 
Table 1. List of studied target compounds. Chromatographic parameters (retention time (Rt), retention index (RI) and quantitation ion (Quan Ion)) obtained from GC-MS chromatograms. Validation parameters obtained after application of optimized procedure (P\&T extraction using EnviCarb $500 \mathrm{mg}$ cartridges, $30 \mathrm{~g}$ of melon sample).

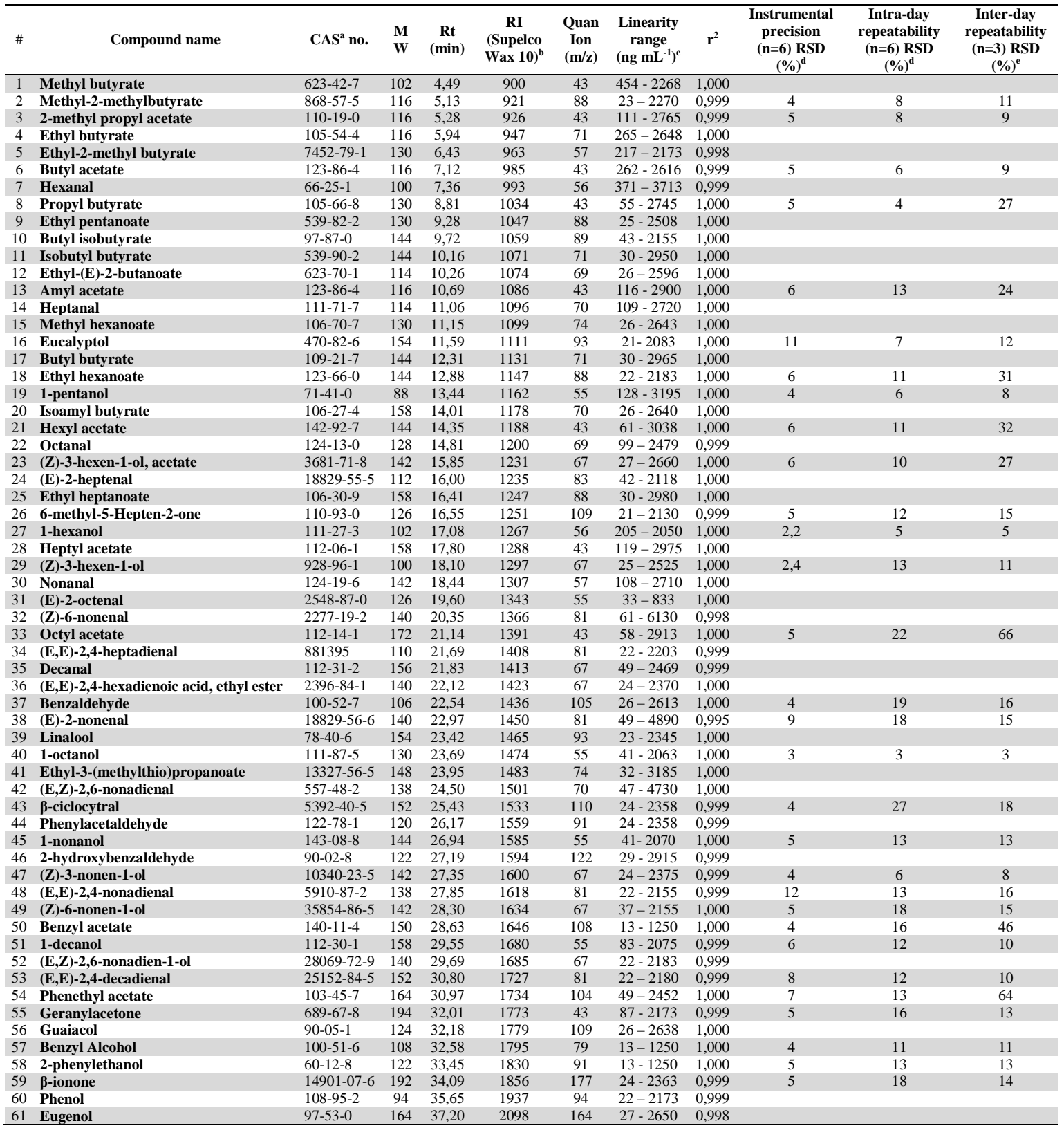

${ }^{a} \mathrm{CAS}=$ Chemical abstract service

${ }^{\mathrm{b}}$ Retention index calculated with n-alkanes on Supelcowax 10 (bonded polyethylene glycol) capillary column.

${ }^{c}$ Linearity range corresponding to the real concentration of standards used for calibration.

${ }^{\mathrm{d}}$ Calculated as relative standard deviation for analysis of six replicates of "Charentais" melon samples.

${ }^{\mathrm{e}} \mathrm{RSD}$ calculated on 3 different days, three replicates per day giving a total of twelve values during one week. 


\subsection{Chemical and supplies}

Reference standards of volatile compounds studied (Table 1) were supplied by Supelco (Sigma-Aldrich and Fluka; Barcelona, Spain) as pure compounds (90-99.5\% purity). GC grade solvents were obtained from Scharlab (Barcelona, Spain). Supelclean ${ }^{\mathrm{TM}}$ ENVICarb $^{\text {TM }}$ 120-400 mesh, $500 \mathrm{mg}$ and TENAX TA ${ }^{\circledR} 60-80$ mesh, $500 \mathrm{mg}$ SPE Tubes $6 \mathrm{~mL}$ (Supelco, Barcelona, Spain) were used as traps. Standard stock solutions (500 $\mathrm{mg} \mathrm{L}^{-1}$ ) of the individual volatile standards were prepared in acetone, from which seven mixed standard solutions (around $50 \mathrm{mg} \mathrm{L}^{-1}$ ) were obtained, by 10-fold volume dilution in acetone. Calibration solutions were prepared from working solutions by consecutive volume dilutions with $n$-hexane to different final concentrations, according to the detector response for each compound (more concentrated when response was lower). All standard solutions were stored at $18{ }^{\circ} \mathrm{C}$ in sealed glass vials (without leaving any headspace) to avoid analyte losses and to ensure reproducibility, as recommended in many references (Kozłowska et al., 2006).

\subsection{Optimised extraction procedures}

The extraction was carried out using a dynamic headspace system (DHS) using a purge \& trap home-made device (Beltran et al., 2006) using commercial (500 mg) SPE cartridges as traps. Before analysis, trap cartridges (EnviCarb SPE $500 \mathrm{mg}$ in the optimized method) were conditioned with $5 \mathrm{~mL}$ of diethyl ether $\left(\mathrm{Et}_{2} \mathrm{O}\right)$, followed by $5 \mathrm{~mL}$ of $\mathrm{n}$-hexane and finally vacuum dried for $10 \mathrm{~min}$. A sample amount of $30 \mathrm{~g}$ of homogenised fruit was weighed into a $150 \mathrm{~mL}$ flask closed with a glass cap with two connection tubes; the inlet tube was connected to a dry nitrogen gas $\left(\mathrm{N}_{2}\right)$ source and the outlet tube to the trap. The extraction time was adjusted to $49 \mathrm{~min}$ using a nitrogen flow rate of $1.6 \mathrm{~L} \mathrm{~min}^{-1}$. Samples were stirred at $300 \mathrm{rpm}$ using a magnetic stir bar and heated at $40^{\circ} \mathrm{C}$.

After extraction, each cartridge was eluted with $5 \mathrm{~mL}$ of diethyl ether/hexane (1:1) followed by $5 \mathrm{~mL}$ of diethyl ether directly into a graduated glass tube. The extract was evaporated under a gentle nitrogen stream at a controlled temperature of $35^{\circ} \mathrm{C}$ to a final volume of $0.5 \mathrm{~mL}$. The final extract was divided in two aliquots in vials with $200 \mu \mathrm{L}$ inserts, sealed and stored in a freezer at $20^{\circ} \mathrm{C}$ until their analysis by GC-MS (ion trap). 
Optimised recommended procedure was developed by applying a multivariate response surface optimisation of the factors considered most important and influencing extraction efficiency. Three factors at three different levels were considered (A: sample weight (g); B: extraction time (minutes); C: gas flow (\% max scale of $\left.1.6 \mathrm{~L} \mathrm{~min}^{-1}\right)$. Sample weight was studied in the range from 5 to $30 \mathrm{~g}$ (levels 5, 17.5 and 30), extraction time from 15 to 60 min (levels 15, 37.5 and 60) and gas flow from 0.16 to $1.6 \mathrm{~mL} \mathrm{~min}^{-1}$ (levels 10, 55 and $100 \%$ max scale). The studied parameter ranges were selected according to previous experience with a similar method developed for tomato samples (Beltran et al., 2006). A Box-Behnken design (Anderson \& Whitcomb, 2005) consisting of 17 experiments (ran in three blocks) was obtained and results obtained after extraction and GC-MS determination were analysed using Design Expert Software (Version 9.0.2, Stat-Ease, Inc., Minneapolis, $\mathrm{MN})$.

Optimisation experiments were carried out using, as indicated above, a "Piel de sapo" muskmelon sample. As response variables, only the twelve most prominent compounds in this muskmelon type were used: (E,Z)-2,6-nonadienal, (Z)-3-hexen-1-ol, (Z)-3-nonen-1-ol acetate, (Z)-6-nonenal, (E)-2-nonenal, (E,Z)-2,6-nonadien-1-ol，(Z)-3-nonen-1-ol, (Z)-6nonen-1-ol, 1-hexanol, 1-nonanol, benzaldehyde and nonanal. A quadratic regression model was used to adjust experimental data and fittings to the data were checked with ANOVA. The optimal extraction conditions were determined using a weighted desirability function together with a simplex algorithm with variable size (Anderson \& Whitcomb, 2005). This desirability function finds the combination of extraction factors which best satisfies the objective fixed for each VOC studied (maximisation of extraction) when considering all the compounds simultaneously. The verification of the validity and adequacy of the predictive extraction model was checked by the application of the optimum extraction conditions selected to six replicate samples and comparing model predictions with experimentally obtained values using a two sided t-test ( $\alpha=0.01)$.

\subsection{GC-MS determination}

A Varian CP-3800 gas chromatograph coupled to a mass spectrometry detector (Saturn 4000, Varian) was used for the determination of volatile compounds in muskmelon and watermelon samples. The analytes were separated on a $30 \mathrm{~m}, 0.25 \mathrm{~mm}$ Supelcowax 10 
(0.25 $\mu \mathrm{m}$ film thickness) capillary column (Supelco, Bellefonte, PA), using helium at a constant flow of $1 \mathrm{~mL} \mathrm{~min}^{-1}$ as carrier gas.

The temperature program was: $40^{\circ} \mathrm{C}$ for $5 \mathrm{~min}$, then increased to $160^{\circ} \mathrm{C}$ at $4{ }^{\circ} \mathrm{C} \mathrm{min}{ }^{-1}$, and finally increased to $250{ }^{\circ} \mathrm{C}$ at $30{ }^{\circ} \mathrm{C} \mathrm{min}-1$, with a final isothermal stage of 1 min (total chromatographic analysis time $39 \mathrm{~min}$ ). Injection of $1 \mu \mathrm{L}$ of sample in the splitless mode (injection port temperature $220{ }^{\circ} \mathrm{C}$ ) was performed using a Varian 8400 autosampler equipped with a $10 \mu \mathrm{L}$ syringe. MS (ion trap) determinations were performed in full scan mode (m/z scan range of 50-200 Da) using electron impact ionization $(70 \mathrm{eV})$ in positive ion mode and external ionisation configuration. GC-MS interface, ion trap and manifold temperatures were set at 275,190 and $60{ }^{\circ} \mathrm{C}$, respectively. Retention indices for all studied compounds were calculated using a standard containing n-alkanes (C7-C30) on a Supelcowax 10 capillary column and following the formula given by Kovats (1958).

Quantitation was carried out by using external standard calibration curves obtained by using peak areas from the corresponding extracted ion chromatograms for the selected quantitation ion (Q) for each compound (Table 1). Compounds with concentration exceeding linearity range were quantified by diluting extracts with $n$-hexane until proper concentration.

\subsection{Validation}

Method validation was carried out by applying a strategy considering linearity, precision (instrumental, intra-day, and inter-day), selectivity, and detection limits, using "Charentais" muskmelon samples. The validation parameters applied were:

Linearity: GC-MS injection of three replicates of the complete calibration curve with seven concentration levels, defined according to expected real sample contents for each compound.

Selectivity: a procedure blank (complete extraction procedure applied over an empty flask) was injected in the GC-MS system.

Instrumental precision: a single sample extract was injected 6 consecutive times.

The intra-day repeatability was determined by extracting and analysing 6 replicates of the same sample under identical experimental conditions on the same day. 
Inter-day reproducibility was performed by analysing 12 replicates of the same sample on three different days in a period of 5 days (6 replicates on day 1, 3 replicates on day 3 and 3 replicates on day 5).

Limits of detection (LOD) were calculated as the lowest concentration corresponding to a peak with a signal/noise ratio of 3 .

\section{RESULTS AND DISCUSSION}

\subsection{GC-MS determination}

Table 1 shows retention time, retention index and quantification $\mathrm{m} / \mathrm{z}$ ions for each studied compound, obtained after injection of solvent standard solutions prepared in n-hexane. The quantification ions were selected according to their abundance in the mass spectrum and their signal-to-noise ratio. As an example Figure 1 shows the chromatograms obtained for (a) a standard mixture of the 61 compounds selected and (b) a "Charentais" muskmelon sample extract. .

\subsection{Extraction optimization}

Once the GC-MS conditions for the selected compounds were obtained the extraction conditions were optimised. Optimisation was divided in two steps: firstly effectiveness of the sorbent type used as trap was studied. Secondly, a more accurate procedure based on response surface method optimisation was applied to obtain the exact extraction conditions that favoured overall extraction of all studied compounds. 

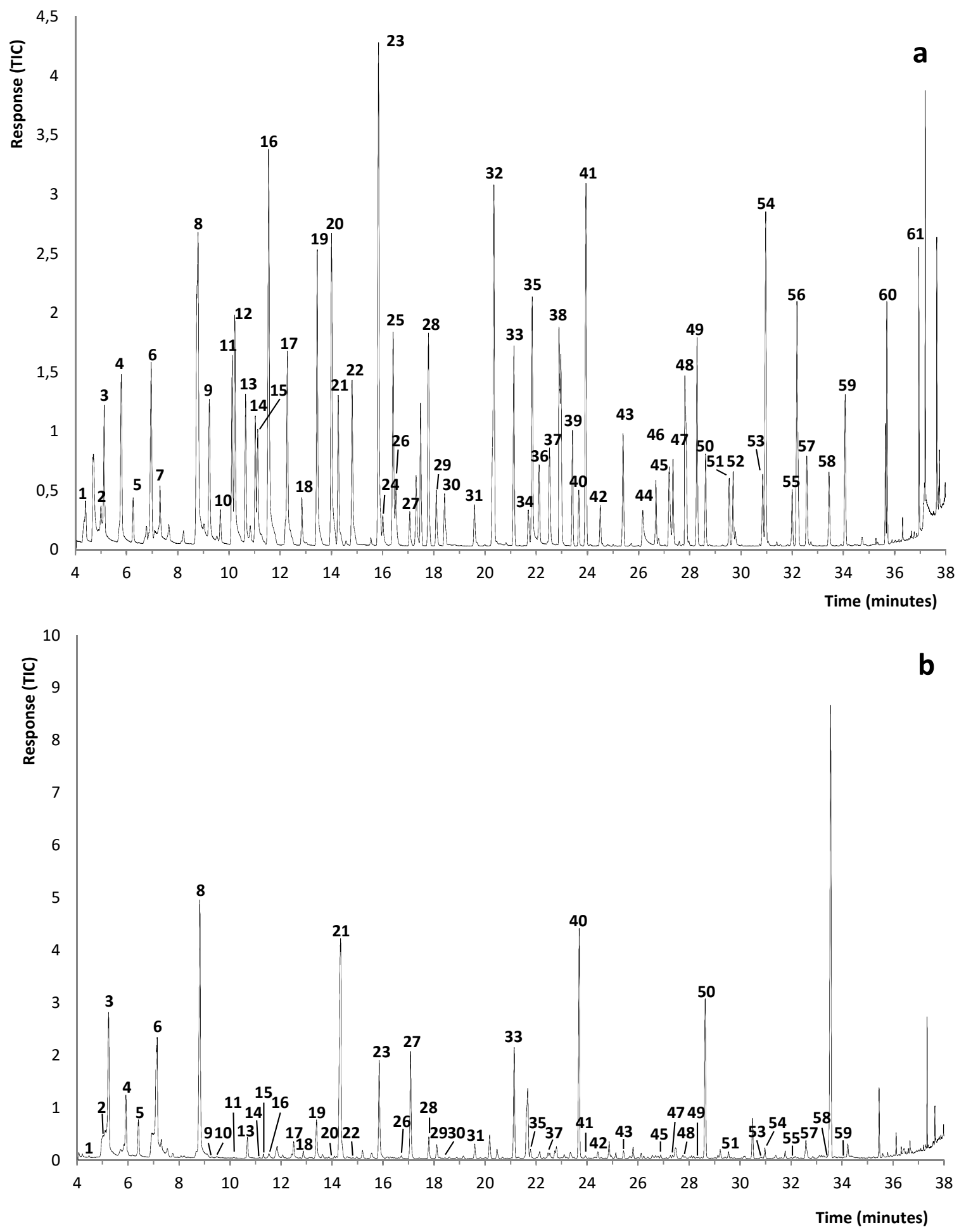

Figure 1. a) Total ion chromatogram (TIC) of a standard mixture of 61 volatile compounds and b) TIC of a Charentais muskmelon sample extract. See Table 1 for peak identification 
In the first step, two commercial adsorbents (500 mg), ENVI-Carb and TENAX, were evaluated applying the purge-and-trap procedure with initial fixed conditions (sample weight $30 \mathrm{~g}$, extraction temperature $40^{\circ} \mathrm{C}$, extraction time $30 \mathrm{~min}$, purge gas flow $1.6 \mathrm{~L}$ $\mathrm{min}^{-1}$ ) according to Beltran et al. (2006). After extraction, each cartridge was eluted with three different solvents of increasing polarity which were collected separately: Fraction 1, elution with $5 \mathrm{~mL}$ hexane-diethyl ether (1:1) mixture, Fraction 2 elution with $5 \mathrm{~mL}$ diethyl ether and Fraction 3 consisting of $5 \mathrm{~mL}$ ethyl acetate. Each fraction collected was concentrated to $0.5 \mathrm{~mL}$ final volume (adding $0.5 \mathrm{~mL}$ of hexane prior to $\mathrm{N}_{2}$ stream evaporation) and injected in the GC-MS system In order to get the maximum information from these experiments, two "Piel de sapo" homogenised muskmelon samples were spiked with a mixture of 61 target compounds giving a final nominal sample concentration of around $50 \mathrm{ng} \mathrm{g}^{-1}$ for each compound. Chromatograms obtained were integrated (using Q ion extracted chromatograms) and the peak areas for each compound in both adsorbents compared. Results indicated that almost all volatile compounds were eluted in the first fraction (Fraction 1), whereas in Fraction 2 ( $5 \mathrm{~mL}$ of diethyl ether) only the most retained compounds were still present corresponding to less than $10 \%$ of peak areas obtained in Fraction 1 (except for 1-pentanol in Tenax cartridge that showed a peak area of around $45 \%$ of the peak area in Fraction 1). In Fraction 3 (5 mL ethyl acetate) none of the compounds studied was detected. Accordingly, selected elution conditions were $5 \mathrm{~mL}$ hexane-diethyl ether (1:1) mixture followed by $5 \mathrm{~mL}$ diethyl ether, collected together and evaporated to a final volume of $0.5 \mathrm{~mL}$.

In relation to the effectiveness of the adsorbents results showed that all target analytes were detected in both cases (Tenax and EnviCarb sorbent extracts). In addition, peak areas obtained for more than $65 \%$ of compounds were of the same order (approximated as the sum areas for both elution fractions). Differences found were especially relevant for 2methylpropyl acetate and methyl 2-methylbutyrate that had signals in EnviCarb of around $40 \%$ and $20 \%$, respectively compared to those obtained in Tenax (values for 2methylpropyl acetate and methyl 2-methylbutyrate were 82,650 and 42,335 in Tenax sorbent, and 34,010 and 8550 for EnviCarb). Although the performance of Tenax sorbent is a little better than EnviCarb, both sorbents allow extraction of all considered compounds, and were considered suitable according to the aims of the developed method. To complete the first stage of the extraction optimisation, a breakthrough volume test was performed by using two cartridges connected sequentially to retain analytes (trap). Eluting the two cartridges separately as indicated before, the results showed that only 8 compounds 
reached the second EnviCarb cartridge and always with reduced signals. With Tenax cartridges, the number of compounds reaching the second cartridge increased to 16.

In conclusion, these results, coupled to the fact that EnviCarb cartridges are much less expensive than those of Tenax (about one third of the price, which is an important factor because in plant breeding programs it is necessary to make hundreds of samples for a complete evaluation of the segregant generations) and their availability (Tenax cartridges are manufactured on demand) led to the EnviCarb cartridge being the option selected to continue the work.

The next step in method development was optimisation of "purge-and-trap" conditions. Taking into account the vast diversity of compounds and their physicochemical characteristics, it is really difficult to find which combination of factors maximises the response (in terms of peak area for all compounds) with the smallest number of simple experiments possible. Therefore, the use of an experimental response surface multivariate design was considered with 3 main factors in the extraction process. These factors were sample weight, extraction time, and nitrogen purge flow. Sample weight was studied in the range of 5 to $30 \mathrm{~g}$, extraction time from 15 to $60 \mathrm{~min}$ and purge flow from 0.16 to $1.6 \mathrm{~mL}$ $\min ^{-1}$. Seventeen extraction experiments were performed using a sample of "Piel de sapo" muskmelon type in a Box- Behnken design (in three blocks). Extracts obtained were analysed by GC-MS and the peak areas for twelve compounds selected according to peak areas and representativeness of the main VOCs groups (aldehydes, alcohols and esters) were obtained. The responses (corresponding to the quantitation ion chromatograms in each case) were used to determine the best mathematical models explaining the variability of the results. All the response surface models developed to explain the influence of the studied factors on extraction efficiency were statistically significant $(\mathrm{p}<0.01)$. The factors (sample weight, extraction time and gas flow) and interactions that influence the extractions performed for each studied volatile compound are summarised in Table 2 (column 2, model adjust group of parameters). For these models, regression coefficients of determination showed that, in general, the modelled response covers the point cloud of the experimental results well or very well (columns 3 and 4 in Table 2) and unexplained point cloud curvatures which might diminish their usefulness do not exist (as shown by the nonexistence of significance between "lack of fit" of residues to the pure error of the model, column 5 in Table 2). Moreover, 10 of the 12 calculated models showed high or very high precision as shown by the signal-to-noise ratio parameter (column 6, Table 2). 
Consequently, we consider that the models can explain the experimental results obtained in a complete way.

Table 2. Results obtained in the optimization of volatiles extraction in muskmelon samples using RSM. Significant model factors, parameters of model goodness of fit and confirmation results in the optimum extraction conditions predicted are indicated.

\begin{tabular}{|c|c|c|c|c|c|c|c|c|c|}
\hline & \multicolumn{5}{|l|}{ Model Adjust } & \multirow[b]{2}{*}{$\begin{array}{l}\text { Predicted } \\
\text { mean }\end{array}$} & \multicolumn{3}{|c|}{ Confirmation trial } \\
\hline & $\begin{array}{l}\text { Significant factors } \\
\text { and interactions } \\
\text { of each RSM } \\
\text { model a }^{\text {a }}\end{array}$ & $\mathbf{R}^{2}$ & $\begin{array}{l}\text { Model } R^{2} \\
\text { adjusted }^{b}\end{array}$ & $\begin{array}{l}\text { "Lack of fit" } \\
\text { significance }\end{array}$ & $\begin{array}{l}\text { Adequate } \\
\text { precision }^{\text {d }}\end{array}$ & & $\begin{array}{l}99 \% \text { PI } \\
\text { low }\end{array}$ & $\begin{array}{l}\text { Observed } \\
\text { mean }\end{array}$ & $\begin{array}{l}\text { 99\% PI } \\
\text { high }\end{array}$ \\
\hline (E,Z)-2,6-nonadienal & $\mathrm{C}, \mathrm{C}^{2}$ & 0,83 & 0,62 & 0,51 & 5,81 & 55099 & 32546 & 363834 & $1,78 \mathrm{E}+12$ \\
\hline (Z)-3-hexen-1-ol & $\mathrm{B}, \mathrm{AB}, \mathrm{B}^{2}, \mathrm{C}^{2}$ & 0,96 & 0,96 & 0,29 & 0,61 & 51998 & 24457 & 34512 & 107857 \\
\hline (E)-2-nonenal & $\mathrm{C}, \mathrm{C}^{2}$ & 0,87 & 0,71 & 0,44 & 6,64 & 10068 & 6184 & 53218 & 61901 \\
\hline (E,Z)-2,6-nonadien-1-ol & $\mathrm{A}, \mathrm{B}, \mathrm{C}^{2}$ & 0,91 & 0,79 & 0,63 & 9,11 & 29926 & 8416 & 91323 & 745203 \\
\hline (Z)-3-nonen-1-ol & $\mathrm{A}, \mathrm{B}, \mathrm{AB}, \mathrm{B}^{2}, \mathrm{C}^{2}$ & 0,97 & 0,93 & 0,41 & 17,78 & 397585 & 228228 & 401428 & 683299 \\
\hline (Z)-6-nonen-1-ol & $\mathrm{B}, \mathrm{B}^{2}, \mathrm{C}^{2}$ & 0,90 & 0,78 & 0,39 & 9,24 & 356696 & 89924 & 296002 & $1,31 \mathrm{E}+06$ \\
\hline 1-hexanol & B & 0,47 & 0,35 & 0,17 & 6,87 & 25584 & 9912 & 18747 & 117278 \\
\hline
\end{tabular}

a Model factors: $\mathrm{A}=$ sample weight $(\mathrm{g}), \mathrm{B}=$ extraction time $(\mathrm{min})$ and $\mathrm{C}=$ gas flow $\left(\%\right.$ max of the scale, $\left.1.6 \mathrm{~L} \mathrm{~min}{ }^{-1}\right)$.

b Regression coefficient of determination corrected to have a meaningful comparison of models with very different numbers of explicative factors.

c Comparisons of the "Lack of fit" of the residuals relative to the model pure error indicates the probability of a curvature not considered in the model could occur. When this parameter is not significant $(\mathrm{P}>0.05)$ the lack of fit could occur due to noise and the model is good.

d The adequate precision measures the signal to noise ratio. A ratio greater than 4 is desirable.

e Anomalous value

The developed models showed that factor A (sample weight) and factor B (extraction time) were the main factors influencing extraction for (Z)-3-hexen-1-ol, (E,Z)-2,6-nonadien-1-ol, (Z)-3-nonen-1-ol, benzaldehyde, (Z)-6-nonen-1-ol, and 1-hexanol. Factors A and C (gas flow (\% max scale; $\left.1.6 \mathrm{~L} \mathrm{~min}^{-1}\right)$ ) were relevant in the extraction of nonanal, (Z)-3-hexen-1ol, and (Z)-6-nonenal; and factors B and $\mathrm{C}$ were important for the rest of the compounds. The magnitude and influence of each factor on each compound extraction can be seen better in the individual graphic representation of the models (Figure 2). In these plots, it is easy to see the conditions that maximise each volatile compound extraction and the maxima of all these models were obtained at different values of the studied factors. So, the determination of a common optimum combination was extremely difficult, since the maximum values of all these models did not coincide. To solve this problem, a weighted 
desirability function (Anderson \& Whitcomb, 2005) targeted to maximise the extraction for each compound was applied. Desirability results indicated that the optimum joint extraction conditions corresponded to $30 \mathrm{~g}$ sample weight, 49 min of extraction time and a gas flow of $1.6 \mathrm{~L} \mathrm{~min}^{-1}$ (marked as solid black lines in the factors plane of each subplot in Figure 2). With the calculated optimal levels for the extraction parameters we can obtain the extraction values expected for each compound (listed numerically as the "predicted mean" column in Table 2).

To check the reliability of the optimised model conditions, an assay was performed by extracting six replicates of a "Piel de sapo" sample with the optimised conditions and subsequent GC-MS determination. Predicted extraction means from the models were used to construct confidence prediction intervals (columns 8 and 10 in Table 2). Actual confirmation values obtained (column 9 in Table 2) generally fall within these confidence intervals and, consequently, there are no significant differences between predicted and observed extraction values. Only benzaldehyde and (Z)-3-hexen-1-ol acetate were exceptions. In the case of benzaldehyde, the result was an anomalous value because other samples of "Piel de sapo" muskmelon analysed under the optimal extraction conditions showed benzaldehyde area values that fit well in the predicted interval. In relation to (Z)-3hexen-1-ol acetate, its value in this confirmatory assay was slightly under the lowest predicted value, but considering that this compound has the narrowest confidence interval (together with benzaldehyde) the model optimisation predictions could be accepted.

In conclusion, the use of $30 \mathrm{~g}$ of weight sample, $49 \mathrm{~min}$ of extraction time with EnviCarb phase, elution with $5 \mathrm{~mL}$ hexane-diethyl ether (1:1) mixture followed by $5 \mathrm{~mL}$ diethyl ether and $1.6 \mathrm{~L} \mathrm{~min}^{-1}$ of nitrogen flow were selected as the best extraction conditions for the purge-and-trap methodology developed in this study. 

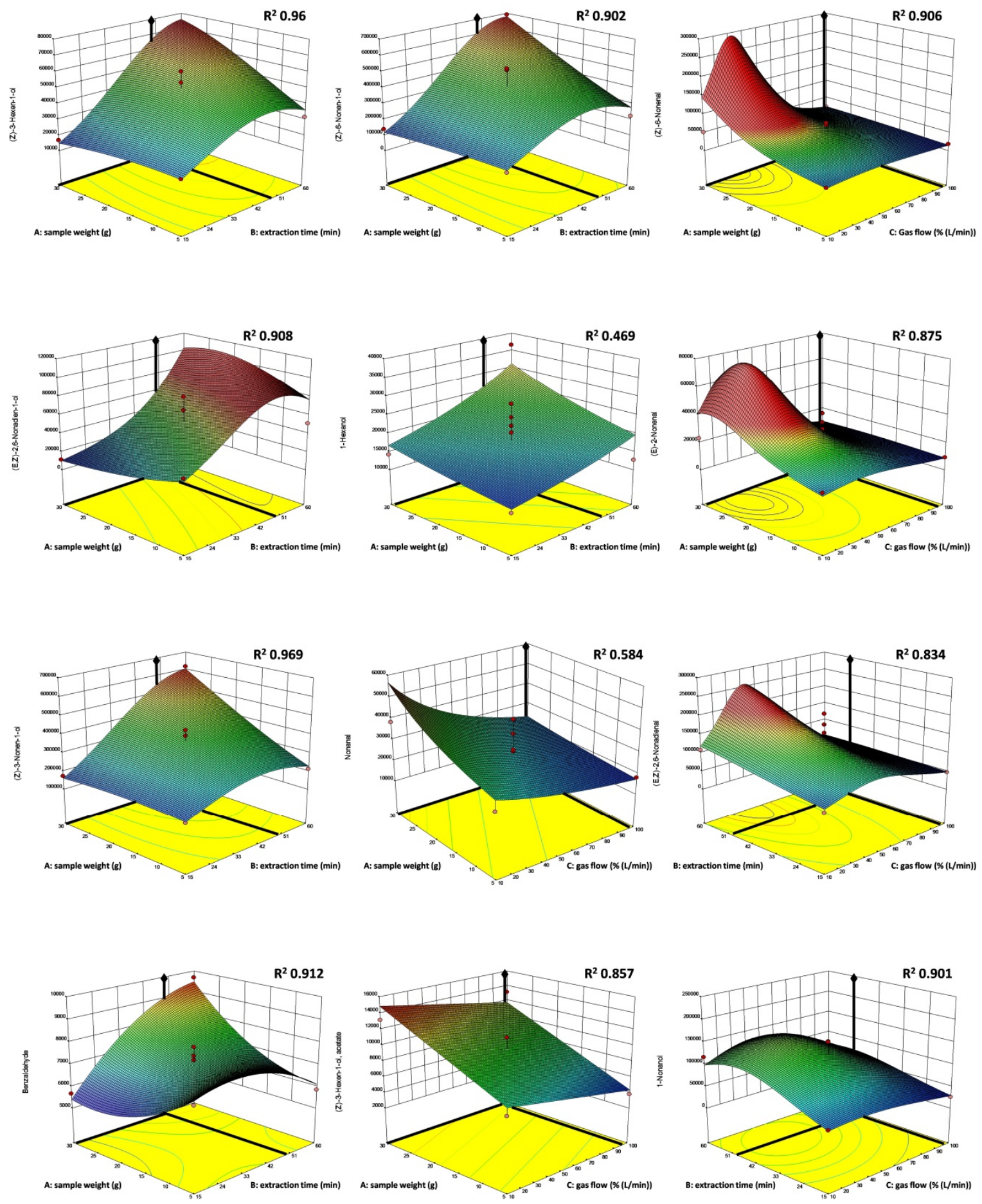

Figure 2. Response surface plots showing the effect of A: sample weight (g), B: extraction time (min) and C: gas flow (\% max of the scale, $1.6 \mathrm{~L}$ min-1) on the peak area of prominent volatile compounds found in muskmelon and watermelon. The selected conditions with the weighted desirability function to maximize global extraction are represented with a vertical black solid line. 


\subsection{Validation}

Once optimised, the method was validated in terms of linearity using solvent standard solutions, and in terms of specificity, precision and detection limit using real samples. Although "Piel de sapo" muskmelon type was used for method optimisation, preliminary experiments showed that "Charentais" muskmelon presented a higher number of volatile compounds compared to the rest of the cultivars, so it was selected for validation studies. Linearity was tested by injecting (in triplicate) 7 concentration level standards ranging from $10 \mathrm{ppb}$ to $5 \mathrm{ppm}$ (nominal concentration). The calibration curves generated by plotting peak areas ( $\mathrm{Q}$ ion signal) versus concentration $\left(\mathrm{ng} \mathrm{mL}^{-1}\right)$ of each standard showed acceptable correlation coefficients $\left(r^{2}\right)>0.995$ for all the compounds studied.

Precision was calculated by obtaining the instrumental, intra-day and inter-day precision (expressed as \% RSD) of peak. Instrumental precision $(n=6)$ ranged from $2 \%$ to $12 \%$. Intra-day $(\mathrm{n}=6)$ precision showed RSD $(\%)$ below $20 \%$ except for octyl acetate and bcyclocitral. As could be expected, the inter-day RSD were higher than intra-day precision, especially for benzyl acetate, hexyl acetate, phenethyl acetate, octyl acetate, propyl butyrate, amyl acetate, (Z)-3-hexen-1-ol acetate, and ethyl hexanoate that showed a RSD greater than $20 \%$. During method development and validation we considered the feasibility of using an internal standard to improve precision and account for recovery, but when using D3-methyl salicylate as a surrogate only a few compounds improved, while the rest of them showed a big deterioration in precision. These RSD results were better than those reported for a liquid-liquid extraction method coupled to GC-MS (Aubert \& Bourger, 2004), and similar to those obtained by our group using the SPME technique for some coincident compounds in tomato samples (Beltran et al., 2006). All the values for precision and linearity are shown in detail in Table 1. Detection limits (LODs) were calculated as the concentration of a peak having a signal/noise ratio of 3 (Table 3 ). 
Table 3.- Real sample determination.

\begin{tabular}{|c|c|c|c|c|c|c|c|c|c|}
\hline \multirow[b]{2}{*}{ \# } & \multirow[b]{2}{*}{$\underset{(\mathbf{m i n})}{\mathbf{R t}}$} & \multirow[b]{2}{*}{ Compound name } & \multicolumn{5}{|c|}{ Muskmelon $\left(\mathrm{ng} \mathrm{g}^{-1}\right)^{\mathrm{a}}$} & \multirow[b]{2}{*}{$\begin{array}{c}\text { Watermelon } \\
\left(\text { ng g g}^{-1}\right)^{\mathrm{a}}\end{array}$} & \multirow[b]{2}{*}{$\underset{\left(\mathbf{n g ~ g}^{-1}\right)}{\text { LOD }}$} \\
\hline & & & Charentais & Tendral & $\begin{array}{c}\text { Piel } \\
\text { de } \\
\text { sapo }\end{array}$ & Galia & Amarillo & & \\
\hline 1 & 4,49 & Methyl butyrate & 17,0 & n.d. & n.d. & n.d. & n.d. & n.d. & 9,7 \\
\hline 2 & 5,13 & Methyl-2-methylbutyrate & 30,4 & n.d. & n.d. & 1,2 & 0,8 & n.d. & 0,9 \\
\hline 3 & 5,28 & 2-methyl propyl acetate & 1805,7 & n.d. & n.d. & 4,8 & 2,9 & n.d. & 1,6 \\
\hline 4 & 5,94 & Ethyl butyrate & 626,9 & n.d. & n.d. & n.d. & n.d. & n.d. & 13,8 \\
\hline 5 & 6,43 & Ethyl-2-methyl butyrate & 248,0 & 8,4 & 8,6 & 9,1 & 9,0 & 8,3 & 1,8 \\
\hline 6 & 7,12 & Butyl acetate & 3038,8 & n.d. & n.d. & n.d. & n.d. & n.d. & 274,2 \\
\hline 7 & 7,36 & Hexanal & n.d. & n.d. & 11,4 & 3,9 & 5,4 & 31,9 & 2,4 \\
\hline 8 & 8,81 & Propyl butyrate & 717,4 & n.d. & n.d. & 2,5 & 1,2 & n.d. & 0,4 \\
\hline 9 & 9,28 & Ethyl pentanoate & 2,3 & n.d. & n.d. & n.d. & n.d. & n.d. & 0,4 \\
\hline 10 & 9,72 & Butyl isobutyrate & 0,7 & n.d. & n.d. & n.d. & n.d. & n.d. & 0,4 \\
\hline 11 & 10,16 & Isobutyl butyrate & 2,6 & n.d. & n.d. & n.d. & n.d. & n.d. & 0,4 \\
\hline 12 & 10,26 & Ethyl-(E)-2-butanoate & 0,6 & n.d. & n.d. & n.d. & n.d. & n.d. & $0,3^{\mathrm{b}}$ \\
\hline 13 & 10,69 & Amyl acetate & 124,5 & n.d. & n.d. & n.d. & n.d. & n.d. & 1,8 \\
\hline 14 & 11,06 & Heptanal & 1,0 & 0,9 & 1,5 & 1,7 & 1,3 & 1,4 & 0,8 \\
\hline 15 & 11,15 & Methyl hexanoate & 2,0 & n.d. & n.d. & n.d. & n.d. & n.d. & 0,1 \\
\hline 16 & 11,59 & Eucalyptol & 1,2 & n.d. & n.d. & n.d. & n.d. & n.d. & 0,2 \\
\hline 17 & 12,31 & Butyl butyrate & 1,1 & n.d. & n.d. & n.d. & n.d. & n.d. & 0,8 \\
\hline 18 & 12,88 & Ethyl hexanoate & 16,7 & n.d. & n.d. & n.d. & n.d. & n.d. & 0,5 \\
\hline 19 & 13,44 & 1-pentanol & 10,3 & 3,9 & 3,4 & 4,4 & 4,0 & 26,9 & 0,8 \\
\hline 20 & 14,01 & Isoamyl butyrate & 0,7 & 0,8 & n.d. & 0,9 & 1,0 & 1,1 & 0,1 \\
\hline 21 & 14,35 & Hexyl acetate & 1679,7 & n.d. & n.d. & 1,2 & n.d. & n.d. & 0,6 \\
\hline 22 & 14,81 & Octanal & 2,1 & 0,8 & 1,3 & 1,1 & 1,2 & 1,5 & 0,2 \\
\hline 23 & 15,85 & (Z)-3-hexen-1-ol acetate & 145,2 & n.d. & n.d. & 0,7 & 0,9 & 0,6 & 0,1 \\
\hline 24 & 16,00 & (E)-2-heptenal & n.d. & n.d. & 2,3 & 0,8 & 2,0 & 4,4 & 0,3 \\
\hline 25 & 16,41 & Ethyl heptanoate & n.d. & n.d. & n.d. & n.d. & n.d. & n.d. & $0,2^{\mathrm{b}}$ \\
\hline 26 & 16,55 & 6-methyl-5-hepten-2-one & 1,0 & 0,9 & 1,0 & 0,9 & 1,0 & 25,0 & 0,1 \\
\hline 27 & 17,08 & 1-hexanol & 491,6 & 6,4 & 2,7 & 3,9 & 2,6 & 151,5 & 0,8 \\
\hline 28 & 17,80 & Heptyl acetate & 42,1 & n.d. & n.d. & n.d. & n.d. & n.d. & 0,6 \\
\hline 29 & 18,10 & (Z)-3-hexen-1-ol & 74,0 & 4,8 & 2,6 & 4,3 & 2,5 & 18,9 & 0,3 \\
\hline 30 & 18,44 & Nonanal & 7,6 & 18,5 & 103,1 & 28,0 & 69,8 & 25,4 & 7,0 \\
\hline 31 & 19,60 & (E)-2-octenal & 47,1 & n.d. & 0,9 & n.d. & 0,4 & 1,8 & 0,3 \\
\hline 32 & 20,35 & (Z)-6-nonenal & n.d. & 7,2 & 62,4 & 22,2 & 48,6 & 6,0 & 0,8 \\
\hline 33 & 21,14 & Octyl acetate & 268,2 & n.d. & n.d. & n.d. & n.d. & n.d. & 3,8 \\
\hline 34 & 21,69 & (E,E)-2,4-heptadienal & n.d. & 0,8 & 1,1 & 0,8 & 1,1 & 1,0 & 0,3 \\
\hline 35 & 21,83 & Decanal & 1,8 & 1,6 & 1,9 & 2,1 & 1,8 & 1,7 & 0,5 \\
\hline 36 & 22,12 & (E,E)-2,4-hexadienoic acid ethyl ester & n.d. & n.d. & n.d. & n.d. & n.d. & n.d. & $0,2^{\mathrm{b}}$ \\
\hline 37 & 22,54 & Benzaldehyde & 9,5 & 1,1 & 1,3 & 1,5 & 4,0 & 1,1 & 0,1 \\
\hline 38 & 22,97 & (E)-2-nonenal & n.d. & 4,0 & 23,7 & 5,1 & 8,0 & 36,1 & 1,3 \\
\hline 39 & 23,42 & Linalool & n.d. & n.d. & n.d. & n.d. & n.d. & n.d. & $0,2^{\mathrm{b}}$ \\
\hline 40 & 23,69 & 1-octanol & 600,2 & 3,1 & 2,0 & 2,2 & 1,9 & 30,8 & 0,7 \\
\hline 41 & 23,95 & Ethyl-3-(methylthio)propanoate & 1,8 & n.d. & n.d. & n.d. & n.d. & n.d. & 0,1 \\
\hline 42 & 24,50 & $(\mathbf{E}, \mathbf{Z})-2,6$-nonadienal & 1,2 & 2,6 & 107,1 & 14,1 & 38,9 & 17,4 & 0,9 \\
\hline 43 & 25,43 & $\beta$-ciclocytral & 0,4 & n.d. & n.d. & n.d. & n.d. & 1,1 & 0,2 \\
\hline 44 & 26,17 & Phenylacetaldehyde & n.d. & n.d. & n.d. & n.d. & n.d. & n.d. & $0,3^{\mathrm{b}}$ \\
\hline 45 & 26,94 & 1-nonanol & 6,4 & 207,0 & 34,5 & 20,7 & 33,6 & 292,4 & 0,7 \\
\hline 46 & 27,19 & 2-hydroxybenzaldehyde & n.d. & n.d. & n.d. & n.d. & n.d. & n.d. & $0,2^{\mathrm{b}}$ \\
\hline 47 & 27,35 & (Z)-3-nonen-1-ol & 5,7 & 138,5 & 118,7 & 91,0 & 15,7 & 2043,9 & 0,2 \\
\hline 48 & 27,85 & (E,E)-2,4-nonadienal & 0,5 & n.d. & n.d. & n.d. & n.d. & 1,1 & 0,2 \\
\hline 49 & 28,30 & (Z)-6-nonen-1-ol & 1,4 & 184,4 & 9,7 & 19,2 & 32,4 & 34,7 & 0,2 \\
\hline 50 & 28,63 & Benzyl acetate & 175,9 & 0,5 & n.d. & 3,3 & 12,5 & n.d. & 0,1 \\
\hline 51 & 29,55 & 1-decanol & 11,2 & 1,1 & n.d. & 0,9 & n.d. & 2,1 & 0,1 \\
\hline 52 & 29,69 & (E,Z)-2,6-nonadien-1-ol & n.d. & 4,4 & 1,7 & 1,1 & 0,9 & 9,7 & 0,7 \\
\hline 53 & 30,80 & (E,E)-2,4-decadienal & 1,0 & n.d. & n.d. & n.d. & n.d. & 2,6 & 0,1 \\
\hline 54 & 30,97 & Phenethyl acetate & 9,9 & n.d. & n.d. & 0,6 & 0,5 & n.d. & 0,1 \\
\hline 55 & 32,01 & Geranylacetone & 2,1 & n.d. & n.d. & n.d. & n.d. & 53,4 & 1,5 \\
\hline 56 & 32,18 & Guaiacol & n.d. & n.d. & n.d. & n.d. & n.d. & n.d. & $0,1^{\mathrm{b}}$ \\
\hline 57 & 32,58 & Benzyl Alcohol & 16,4 & 0,6 & 0,7 & 0,7 & 1,1 & 1,6 & 0,2 \\
\hline 58 & 33,45 & 2-phenylethanol & 2,6 & n.d. & n.d. & n.d. & n.d. & n.d. & 0,1 \\
\hline 59 & 34,09 & $\beta$-ionone & 0,8 & 0,2 & 0,2 & 0,2 & 0,3 & 2,6 & 0,1 \\
\hline 60 & 35,65 & Phenol & n.d. & n.d. & n.d. & n.d. & n.d. & n.d. & $0,2^{\mathrm{b}}$ \\
\hline 61 & 37,20 & Eugenol & n.d. & n.d. & n.d. & n.d. & n.d. & n.d. & $0,2^{\mathrm{b}}$ \\
\hline
\end{tabular}

a Values expressed in $\mathrm{ng} \mathrm{g}^{-1}$ fresh weight.

b These values were estimated from the lowest point of the calibration curves.

n.d. this compound was not detected in real samples. 


\subsection{Determination of real samples}

The applicability of the developed methodology was tested by extracting a number of muskmelon and watermelon samples, which were expected to have different volatile profiles. For this purpose 5 muskmelon types ("Charentais', "Galia', "Piel de Sapo", "Tendral" and "Amarillo") and one watermelon cultivar were evaluated.

Figure 3 and Table 3 show results obtained by applying the developed dynamic headspace purge-and-trap methodology making use of EnviCarb cartridges with the optimised extraction conditions. As was expected, a strong genotypic profile effect was observed, with changes both in single and relative analyte contents in the muskmelon types or watermelon analysed. "Charentais" muskmelon showed a more complex volatile profile when compared with other muskmelon types, with 47 compounds detected, while the rest had around or slightly below 30 compounds detected (Table 3, column 4-9). All these samples contain ethyl 2-methylbutyrate (Z)-3-nonen-1-ol, (Z)-6-nonen-1-ol and (E,Z)-2,6nonadienal, which are reported to have a muskmelon-like flavour (Saftner et al., 2006; Verzera et al., 2011). Several studies reported that 2-methylpropyl acetate, ethyl 2methylbutyrate, ethyl butyrate, ethyl hexanoate, butyl acetate, hexyl acetate, 1-hexanol, (Z)-3-hexen-1-ol, ethyl 3-(methylthio)propanoate, and benzyl acetate were the main organic volatiles observed in some cultivars of "Charentais" muskmelons (Cucumis melo var cantalupensis) in concentration ranges similar to the ones found in this study (Aubert \& Bourger, 2004; Kourkoutas et al., 2006; Obando-Ulloa et al., 2008). The "Charentais" melon profile was differentiated from the others by the presence of butyl acetate, ethyl 3(methylthio)propanoate, ethyl butyrate, amyl acetate, heptyl acetate, methyl butyrate, 2phenylethanol, ethyl hexanoate, phenethyl acetate, methyl hexanoate, isobutyl butyrate, butyl isobutyrate and octyl acetate. Geranylacetone was only present in watermelon samples and compounds like (Z)-3-nonen-1-ol, b-ionone, (E)-2-heptenal and (E,Z)-2,6nonadien-1-ol were present at higher concentrations in watermelon compared to other samples. "Tendral'" muskmelon type contained the highest concentration of (Z)-6-nonen1-ol. "Piel de sapo" muskmelon type presented the highest concentration of (E,Z)-2,6nonadienal and (Z)-6-nonenal.

Regarding the analytical methodology used to evaluate aromatic profiles, in the literature several works can be found dealing with SPME, in which only semiquantitative data, expressed as peak area or percentages (Beaulieu \& Grimm, 2001; Kourkoutas et al., 2006; 
Lamikanra \& Richard, 2002; Obando-Ulloa et al., 2008; Saftner et al., 2006) are reported, making use of a single external standard (Beaulieu, 2005) to calculate relative responses or by using standard additions (Verzera et al., 2011). In our opinion, the method developed in the present study has improved compound quantitation allowing the use of external calibration with a mixture of standards in pure solvents, avoiding the need for using standard additions method or matrix matched calibration. Recently stir-bar sorptive extraction (SBSE) (Amaro et al., 2012) has been used to carry out studies in order to differentiate samples through similar quantitation, using external standard calibration. However, the application of this technique requires the use of specialised equipment. Additionally, as the validation of the method has not been thoroughly discussed by Amaro et al. (2012) it is not easy to compare both methods. Advantages of the proposed purgeand-trap technique compared to SPME and SBSE are: the possibility of extracting several samples at the same time, saving analysis time and reducing overall costs and the fact that a final volume of extract compatible with every GC system is obtained allowing it to be stored and injected several times and in different systems, e.g., high resolution mass spectrometry, for further elucidation of unknowns in retrospective analysis. Our laboratory configuration permitted the extraction of up to 6 samples at a time; but this is completely scalable and could be upgraded to more than 15 at a time in routine laboratories.

The method developed is useful, reproducible and advantageous compared with previous methods, by being easier, less expensive and with higher sample throughput. The data obtained agreed with that in the literature, and thus suggested that the method is a relevant tool to differentiate the volatile profiles in muskmelon and watermelon breeding programs.

\section{ACKNOWLEDGEMENTS}

This study was supported by P1-1B2011-41 and P1-1B2013-70 projects funded by Universitat Jaume I research promotion plans. The authors also acknowledge the financial support of Generalitat Valenciana (research group of excellence PROMETEO/2009/054; ISIC 2012/016 and 'SSantiago Grisolia/2013/032'” Grant for A. Fredes). 


\section{APPENDIX A. Supplementary data}

Table S1. Review of the volatiles compounds presented in melon samples reported in eleven articles. The table shown the reported compounds, CAS numbers, references with the concentration (in the cases that they are quantitate) and comments about sensory descriptors or significant features.

\begin{tabular}{|c|c|c|c|c|}
\hline$\#$ & Compounds & $\mathbf{C A S}^{\mathbf{a}}$ & (Reference) Conc. (units) explanation & Comments \\
\hline 1 & (E)-2-butenal & $123-73-9$ & (11) $14,8(\mu \mathrm{g} / \mathrm{kg})^{\mathbf{i}}$ & \\
\hline 2 & $\begin{array}{l}\text { (E)-2-decenal (in Beaulieu 2001, } \\
\text { 2007; (E)-2-decanal) } \\
\end{array}$ & $3913-81-3$ & (1) n.q. & \\
\hline 3 & (E)-2-heptenal & $18829-55-5$ & (1) n.q.; (9) 0,1(\%area) ${ }^{g}$ & \\
\hline 4 & (E)-2-hexen-1-ol & $928-95-0$ & (9) tr. $^{\mathrm{g}}$ & \\
\hline 5 & (E)-2-hexenal & $6728-26-3$ & $\begin{array}{l}\text { (1) n.q.; (2) n.q.; (4) } 1,9(\mu \mathrm{g} / \mathrm{kg})^{\mathbf{c}} ; \text { (9) } \\
0,2(\% \text { area) })^{\mathbf{g}} ;(\mathbf{1 1}) 14,1(\mu \mathrm{g} / \mathrm{kg})^{\mathbf{i}}\end{array}$ & Main melon aroma \\
\hline 6 & (E)-2-nonenal & $18829-56-6$ & $\begin{array}{l}\text { (1) } 49172(\text { area })^{\mathbf{b}} ; \text { (2) n.q.; (6) 3-10(rel. to } \\
\text { 100ng })^{\mathbf{d}} ;(\mathbf{9}) 2,3(\% \text { area })^{\mathbf{g}} ;(\mathbf{1 0}) 2,7-6,9(\mathrm{pA})^{\mathbf{h}} ; \text { (11) } \\
7,9-61,6(\mu \mathrm{g} / \mathrm{kg})^{\mathbf{i}}\end{array}$ & Adheyde, soapy; Melon \\
\hline 7 & (E)-2-octen-1-ol & $18409-17-1$ & (9) tr. ${ }^{\mathrm{g}}$ & \\
\hline 8 & (E)-2-octenal & $2548-87-0$ & (1) $2088(\text { area })^{\mathbf{b}}$; (2) n.q. & \\
\hline 9 & (E)-2-pentenal & $1576-87-0$ & (4) $7,6(\mu \mathrm{g} / \mathrm{kg})^{\mathrm{c}}$ & \\
\hline 10 & $\begin{array}{l}\text { (E)-2-undecenal (in Beaulieu 2001, } \\
\text { 2007; (E)-2-undecanal) }\end{array}$ & $53448-07-0$ & (1) n.q.; (2) n.q. & \\
\hline 11 & (E)-3-hexen-1-yl acetate & $3681-82-1$ & (1) n.q.; (2) n.q.; (6) 1-25(rel. to 100ng) ${ }^{\mathbf{d}}$ & \\
\hline 12 & (E)-6-nonen-1-ol & $31502-19-9$ & (9) $0,1(\% \text { area })^{\mathrm{g}}$ & \\
\hline 13 & (E)-6-nonenal & $2277-20-5$ & (9) tr. ${ }^{\mathrm{g}}$ & Sweet, creamy \\
\hline 14 & (E)-cinnamic aldehyde & 14371-10-9 & (3) n.q. & \\
\hline 15 & (E)-citral & $141-27-5$ & (1) n.q.; (2) n.q. & \\
\hline 16 & $(\mathrm{E}, \mathrm{E})$-2,4-decadienal & $25152-84-5$ & (1) n.q.; (9) tr. $^{\mathrm{g}}$ & Fatty \\
\hline 17 & (E,E)-2,4-heptadien-1-ol & $33467-79-7$ & (9) $\operatorname{tr}^{\mathrm{g}}$ & \\
\hline 18 & (E,E)-2,4-heptadienal & 4313-03-05 & (1) n.q.; (9) 0,2(\%area $)^{\mathbf{g}}$ & \\
\hline 19 & (E,E)-2,4-nonadienal & $5910-87-2$ & (1) n.q.; (2) n.q.; (9) n.q. & Fatty, fried \\
\hline 20 & (E,E)-2,6-farnesol & $106-28-5$ & (9) $0,4(\% \text { area })^{\mathrm{g}}$ & \\
\hline 21 & (E,E)-3,5-octadien-2-one & $30086-02-3$ & (1) n.q. & \\
\hline 22 & $(\mathrm{E}, \mathrm{E}, \mathrm{Z})-\mathbf{1 , 3 , 5 , 8 - u n d e c a t e t r a e n e}$ & $81717-82-0$ & (1) n.q. & \\
\hline 23 & (E,Z)-2,4-decadienal & $25152-83-4$ & (1) n.q. & \\
\hline 24 & (E,Z)-2,4-heptadienal & $4313-02-04$ & (1) n.q.; (9) 0,1(\%area $)^{\mathrm{g}}$ & \\
\hline 25 & (E,Z)-2,4-nonadienal & $21661-99-4$ & (1) n.q.; (2) n.q. & \\
\hline 26 & (E,Z)-2,6-nonadien-1-ol & 28089-72-9 & (9) $12,2(\% \text { area })^{\mathrm{g}}$ & \\
\hline 27 & (E,Z)-2,6-nonadienal & $557-48-2$ & 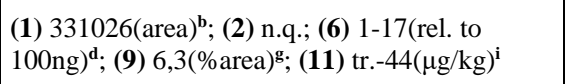 & $\begin{array}{l}\text { Melon, cucumber; High } \\
\text { aroma value }\end{array}$ \\
\hline 28 & (E,Z)-3,6-nonadien-1-ol & $56805-23-3$ & (9) $5,7(\% \text { area })^{\mathrm{g}}$; (11) $104(\mu \mathrm{g} / \mathrm{kg})^{\mathrm{i}}$ & \\
\hline 29 & (E,Z,Z)-2,4,7-decatrien-1-ol & not found & (9) $1,8(\% \text { area })^{\mathrm{g}}$ & \\
\hline 30 & (Z)-2-nonenal & $60784-31-8$ & (9) n.q. & Soapy, fatty \\
\hline 31 & (Z)-3-hepten-1-ol & $1708-81-2$ & (9) $0,2(\% \text { area })^{g}$ & \\
\hline 32 & (Z)-3-hepten-1-yl acetate & $1576-78-9$ & (6) $3-8$ (rel. to $100 \mathrm{ng})^{\mathbf{d}}$ & \\
\hline
\end{tabular}




\begin{tabular}{|c|c|c|c|c|}
\hline 33 & (Z)-3-hexen-1-ol & $928-96-1$ & $\begin{array}{l}\text { (1) n.q.; (3) n.q.; (4) } 6,9(\mu \mathrm{g} / \mathrm{kg})^{\mathbf{c}} ; \text { (5) } 2 \text { (ppm); } \\
\text { (9) } 0,2\left(\% \text { areag) } ; \text { (11) } 120(\mu \mathrm{g} / \mathrm{kg})^{\mathrm{i}}\right.\end{array}$ & Herbal, green \\
\hline 34 & (Z)-3-hexen-1-ol acetate & $3681-71-8$ & $\begin{array}{l}\text { (1) } 3334976(\text { area) })^{\mathbf{b}} ;(\text { (2) n.q.; (3) n.q.; (4) } \\
28,8(\mu \mathrm{g} / \mathrm{kg})^{\mathbf{c}} ; \mathbf{( 5 )} 0,27(\mathrm{ppm}) ;(\mathbf{6}) 32-583 \text { (rel. to } \\
\text { 100ng) })^{\mathbf{d}} ;(\mathbf{1 0}) 3-145,2(\mathrm{pA})^{\mathbf{h}}\end{array}$ & $\begin{array}{l}\text { Green, herbal, banana; } \\
\text { Higher concentration; } \\
\text { Green, fruity }\end{array}$ \\
\hline 35 & (Z)-3-hexenal & $6789-80-6$ & (1) n.q.; (4) $1,2(\mu \mathrm{g} / \mathrm{kg})^{\mathbf{c}}$; (9) n.q. & Green; Main melon aroma \\
\hline 36 & (Z)-3-hexenyl pentanoate & $35852-46-1$ & (3) n.q. & \\
\hline 37 & (Z)-3-nonen-1-ol & $10340-23-5$ & $\begin{array}{l}\text { (5) n.d.; (6) 24-78(rel. to 100ng) })^{\text {d }} \text {; (9) } \\
3,1(\% \text { area })^{\mathbf{g}} ;(\mathbf{1 0})<0,3-2,7(\mathrm{pA})^{\mathbf{h}} ; \text { (11) } 9,6- \\
251(\mu \mathrm{g} / \mathrm{kg})^{\mathbf{i}}\end{array}$ & $\begin{array}{l}\text { Green, pungent, floral; } \\
\text { Melon }\end{array}$ \\
\hline 38 & (Z)-3-nonen-1-yl acetate & $13049-88-2$ & (6) $60-200$ (rel. to $100 \mathrm{ng})^{\mathrm{d}} ;(\mathbf{1 0}) 1,2-53,6(\mathrm{pA})^{\mathbf{h}}$ & \\
\hline 39 & (Z)-3-octen-1-ol & $20125-84-2$ & (1) n.q.; (5) n.d. & Toasted nut, smoky, dusty \\
\hline 40 & (Z)-3-octen-1-ol acetate & $69668-83-3$ & (1) n.q.; (5) 0,13 (ppm); (6) 2-12(rel. to 100ng) ${ }^{\mathbf{d}}$ & \\
\hline 41 & (Z)-4-hepten-1-yl acetate & not found & (6) 7-86(rel. to $100 \mathrm{ng})^{\mathbf{d}}$ & \\
\hline 42 & (Z)-4-heptenal & $6728-31-0$ & (9) n.q. & Fishy \\
\hline 43 & (Z)-5-octen-1-yl acetate & $71978-00-2$ & (6) $1-4$ (rel. to $100 \mathrm{ng})^{\mathbf{d}}$ & \\
\hline 44 & (Z)-6-nonen-1-ol & $35854-86-5$ & 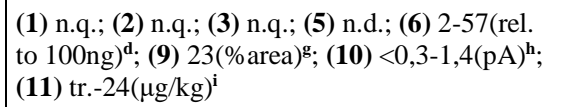 & Green, vegetative; Melon \\
\hline 45 & (Z)-6-Nonen-1-yl acetate & $76238-22-7$ & (6) 7-141(rel. to 100ng) $;$; (10) $2,5-42,6(\mathrm{pA})^{\mathbf{h}}$ & Honeydew \\
\hline 46 & (Z)-6-nonenal & $2277-19-2$ & 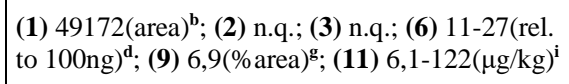 & Melon \\
\hline 47 & (Z)-7-decen-5-olide & $25524-95-2$ & (4) $3,4(\mu \mathrm{g} / \mathrm{kg})^{\mathbf{c}}$ & \\
\hline 48 & (Z)-9-octadecenoic acid & $112-80-1$ & (3) n.q. & \\
\hline 49 & (Z)-citral & $106-26-3$ & (1) n.q.; (2) n.q. & \\
\hline 50 & (Z,Z)-2,6-nonadienal (tentative) & not found & (9) $0,1(\% \text { area })^{\mathrm{g}}$ & Melon \\
\hline 51 & (Z,Z)-3,6-nonadien-1-ol & $53046-97-2$ & (3) n.q.; (6) 9-29(rel. to 100ng) ${ }^{\text {d; }}$ (9) 5,7(\%area) $)^{\mathrm{g}}$ & Fresh; High aroma value \\
\hline 52 & (Z,Z)-3,6-nonadienyl acetate & 83334-93-4 & $\begin{array}{l}\text { (3) n.q.; (6) 76-118(rel. to 100ng) })^{\text {di }} \text { (10) 2,6- } \\
25,4(\mathrm{pA})^{\mathrm{h}}\end{array}$ & \\
\hline 53 & 1,10-undecadiene & $13688-67-0$ & (1) n.q. & \\
\hline 54 & 1,2-dimethylbenzene & $95-47-6$ & (8) $0,5(\text { rel area })^{\mathbf{f}}$ & \\
\hline 55 & 1,4-dimethylbenzene & $106-42-3$ & (8) $1,1(\text { rel area })^{\mathbf{f}}$ & \\
\hline 56 & $\begin{array}{l}\text { 1,7,7-Trimethylnorbonan-2-one } \\
\text { (camphor) }\end{array}$ & $464-48-2$ & (7) $0,4-4(\% \text { A rel. })^{\mathbf{e}} ;(\mathbf{8}) 21(\text { rel area })^{\mathbf{f}}$ & \\
\hline 57 & 1,8-cineol o eucalyptol & $470-82-6$ & $\begin{array}{l}\text { (1) n.q.; (2) n.q.; (3) n.q.; (5) tr.; (6) tr.-16(rel. to } \\
\text { 100ng) }{ }^{\text {d. }} ;(\mathbf{1 1}) 38,5(\mu \mathrm{g} / \mathrm{kg})^{\mathrm{i}}\end{array}$ & \\
\hline 58 & 1-butanol & $71-36-3$ & (5) $0,42(\mathrm{ppm}) ;(\mathbf{1 1}) 45,1(\mu \mathrm{g} / \mathrm{kg})^{\mathbf{i}}$ & \\
\hline 59 & 1-decanol & $112-30-1$ & (9) tr. $^{\mathrm{g}}$ & \\
\hline 60 & 1-heptanol & $111-70-6$ & $\begin{array}{l}\text { (1) n.q.; (4) } 21,9(\mu \mathrm{g} / \mathrm{kg})^{\mathbf{c}} ; \text { (5) n.d.; (11) } \\
32,5(\mu \mathrm{g} / \mathrm{kg})^{\mathrm{i}}\end{array}$ & \\
\hline 61 & 1-hexanol & $111-27-3$ & $\begin{array}{l}\text { (1) n.q.; (3) n.q.; (4) } 20,7(\mu \mathrm{g} / \mathrm{kg})^{\mathbf{c}} ;(\mathbf{5}) 1,09 \\
\text { (ppm); (6) 2-6(rel. to } 100 \mathrm{ng})^{\mathbf{d}} ; \text { (9) 0,2(\%area) } \\
\text { (11) tr.-618( }(\mu \mathrm{g} / \mathrm{kg})^{\mathrm{i}}\end{array}$ & \\
\hline 62 & 1-methoxypropan-2-yl acetate & $108-65-6$ & (8) $0,7(\text { rel area })^{\mathbf{f}}$ & \\
\hline 63 & 1-methylethyl dodecanoate & $10233-13-3$ & (3) n.q. & \\
\hline
\end{tabular}


Capítulo 1

\begin{tabular}{|c|c|c|c|c|}
\hline 64 & 1-nonanol & $143-08-8$ & $\begin{array}{l}\text { (1) n.q.; (2) n.q.; (3) n.q.; (6) 4-48(rel. to } \\
\text { 100ng) }{ }^{\mathbf{d}} ; \text { (9) } 13,5\left(\% \text { area) }{ }^{\mathbf{g}} ; \text { (11) } 42,7-53(\mu \mathrm{g} / \mathrm{kg})^{\mathbf{i}}\right.\end{array}$ & Fatty \\
\hline 65 & 1-nonen-4-ol & $35192-73-5$ & (3) n.q. & \\
\hline 66 & 1-octanol & $111-87-5$ & $\begin{array}{l}\text { (1) n.q.; (3) n.q.; (4) } 122,4(\mu \mathrm{gg} / \mathrm{kg})^{\mathbf{c}} ; \text { (5) n.d.; }(\mathbf{6}) \\
\text { 2-4(rel. to 100ng) })^{\mathbf{d}} ; \mathbf{( 9 )} 0,1(\% \text { area } \\
1,2(\mathrm{pA})^{\mathbf{h}} ;(\mathbf{1 1}) \text { tr.-201( }(\mu \mathrm{g} / \mathrm{kg})^{\mathbf{i}}\end{array}$ & Fatty \\
\hline 67 & 1-octen-3-ol & $3391-86-4$ & $\begin{array}{l}\text { (1) n.q.; (3) n.q.; (4) } 1,2(\mu \mathrm{g} / \mathrm{kg})^{\mathbf{c}} ;(\mathbf{6}) 4-5 \text { (rel. to } \\
\text { 100ng) })^{\mathbf{d}} ;(\mathbf{9}) 0,5\left(\% \text { area) }{ }^{\mathrm{g}} ;(\mathbf{1 1}) \text { tr. }(\mu \mathrm{g} / \mathrm{kg})^{\mathrm{i}}\right.\end{array}$ & Earthy \\
\hline 68 & 1-octen-3-one & $4312-99-6$ & (1) n.q.; (9) n.q. & Mushroom \\
\hline 69 & 1-octen-3-yl acetate & 198242 & (3) n.q.; (6) 4-28(rel. to 100ng) ${ }^{\text {d }}$ & \\
\hline 70 & 1-pentanol & 71-41-0 & $\begin{array}{l}\text { (1) n.q.; (5) n.d.; (9) } 0,4(\% \text { area })^{\mathbf{g}} ; \text { (11) } \\
11,7(\mu \mathrm{g} / \mathrm{kg})^{\mathrm{i}}\end{array}$ & acid, pungent \\
\hline 71 & 1-penten 3-ol & $616-25-1$ & (11) $\operatorname{tr} .(\mu \mathrm{g} / \mathrm{kg})^{\mathbf{i}}$ & \\
\hline 72 & 2-(methylthio)ethanol & $5271-38-5$ & (3) n.q.; (5) 1,97 (ppm) & \\
\hline 73 & 2-(methylthio)ethyl acetate & $5862-47-5$ & (3) n.q.; (4) $102,5(\mu \mathrm{g} / \mathrm{kg})^{\mathbf{c}} ;$; (6) $5(\text { rel. to } 100 \mathrm{ng})^{\mathbf{d}}$ & \\
\hline 74 & 2,3-butanediol & $513-85-9$ & (5) $0,47(\mathrm{ppm})$ & \\
\hline 75 & $\begin{array}{l}\text { 2,3-butanediol diacetate o butane- } \\
\text { 2,3-diol diacetate }\end{array}$ & $1114-92-7$ & $\begin{array}{l}\text { (1) n.q.; (3) n.q.; (4) 56181( } \mu \mathrm{g} / \mathrm{kg})^{\mathbf{c}} ; \text { (5) } 0,23 \\
\text { (ppm); (6) 7-21(rel. to 100ng) }\end{array}$ & \\
\hline 76 & 2,4,6-nonatrienal (tentative) & $100113-52-8$ & (9) $0,1(\% \text { area })^{\mathrm{g}}$ & \\
\hline 77 & 2,5-octanedione & $3214-41-3$ & (1) n.q. & \\
\hline 78 & 2-acetyl furan & $1192-62-7$ & (1) n.q. & \\
\hline 79 & 2-butanone-3-hydroxybutanoate & not found & (3) n.q. & \\
\hline 80 & 2-ethylbutanal & $97-96-1$ & (3) n.q. & \\
\hline 81 & 2-ethylfuran & $3208-16-0$ & (7) $0,5-3(\% \text { A rel. })^{\mathrm{e}}$ & \\
\hline 82 & 2-ethylhexan-1-ol & $104-76-7$ & (7) $0,2-1,2(\% \text { A rel. })^{\mathbf{e}} ;$ (11) $11,6-44,9(\mu \mathrm{g} / \mathrm{kg})^{\mathbf{i}}$ & \\
\hline 83 & 2-ethylhexyl acetate & $103-09-3$ & (6) 3-8(rel. to 100ng) ${ }^{\mathbf{d}}$ & \\
\hline 84 & 2-furanmethanol acetate & $623-17-6$ & (1) n.q. & \\
\hline 85 & 2-methyl 1-butanol & $137-32-6$ & $\begin{array}{l}\text { (1) n.q.; (2) n.q.; (4) 22,8( }(\mu \mathrm{g} / \mathrm{kg})^{\mathrm{c}} ; \text { (5) } 0,76 \\
(\mathrm{ppm}) ;(\mathbf{1 1}) 38,6-148(\mu \mathrm{g} / \mathrm{kg})^{\mathrm{i}}\end{array}$ & \\
\hline 86 & 2-methyl butylbutanoate & $51115-64-1$ & (1) n.q.; (2) n.q.; (6) 2-14(rel. to 100ng) ${ }^{\mathbf{d}}$ & High aroma value \\
\hline 87 & 2-methyl-2-butenoate & $623-43-8$ & (3) n.q. & \\
\hline 88 & 2-methyl-2-butenyl acetate & 19248-94-3 & (6) $2-11$ (rel. to $100 \mathrm{ng})^{\text {d }}$ & \\
\hline 89 & 2-methyl-3-buten-2-ol & $115-18-4$ & (5) $0,79(\mathrm{ppm})$ & \\
\hline 90 & 2-methylbutanal & $96-17-3$ & (11) $\operatorname{tr} .-9,3(\mu \mathrm{g} / \mathrm{kg})^{\mathbf{i}}$ & \\
\hline 91 & 2-methylbutyl 2-methylbutanoate & $2445-78-5$ & (6) $1-8$ (rel. to $100 \mathrm{ng})^{\mathbf{d}} ;(\mathbf{1 1}) \operatorname{tr} .(\mu \mathrm{g} / \mathrm{kg})^{\mathbf{i}}$ & \\
\hline 92 & \begin{tabular}{|l|} 
2-methylbutyl 2- \\
methylpropanoate o 2- \\
methylbutyl isobutyrate \\
\end{tabular} & $2445-69-4$ & (1) n.q.; (6) 4-14(rel. to 100ng) ${ }^{\mathbf{d}}$ & \\
\hline 93 & 2-Methylbutyl acetate & $624-41-9$ & $\begin{array}{l}\text { (1) n.q.; (2) n.q.; (5) } 0,33(\mathrm{ppm}) ;(\mathbf{6}) 485- \\
1578(\text { rel. to } 100 \text { ng })^{\mathbf{d}} ;(\mathbf{1 0}) 13-269,9(\mathrm{pA})^{\mathbf{h}}\end{array}$ & $\begin{array}{l}\text { Fresh, vegetable, banana; } \\
\text { Higher concentration; } \\
\text { Fruity, sweet, banana }\end{array}$ \\
\hline 94 & 2-methylbutyl propanoate & $2438-20-2$ & (6) 9-22(rel. to 100ng) ${ }^{\mathbf{d}}$ & \\
\hline 95 & 2-methylpentane & $107-83-5$ & (8) $2,4(\text { rel area })^{\mathbf{f}}$ & \\
\hline 96 & $\begin{array}{l}\text { 2-methylpropanal o } \\
\text { Isobutyraldehyde }\end{array}$ & $78-84-2$ & (11) $8,5(\mu \mathrm{g} / \mathrm{kg})^{\mathbf{i}}$ & \\
\hline 97 & 2-methylpropanol o isobutanol & $78-83-1$ & (4) $8,5(\mu \mathrm{g} / \mathrm{kg})^{\mathbf{c}} ;$ (5) $2,3(\mathrm{ppm})$ & \\
\hline 98 & $\begin{array}{l}\text { 2-methylpropyl acetate o Isobutyl } \\
\text { acetate }\end{array}$ & $110-19-0$ & $\begin{array}{l}\text { (1) n.q.; (2) n.q.; (3) n.q.; (5) } 0,54(\mathrm{ppm}) ;(\mathbf{6}) \\
\text { 126-615(rel. to } 100 \mathrm{ng})^{\mathbf{d}} ;(\mathbf{7}) 1,2-18,4(\% \text { A rel. })^{\mathrm{e}} \text {; } \\
\text { (10) } 0,4-82,6(\mathrm{pA})^{\mathbf{h}}\end{array}$ & $\begin{array}{l}\text { floral; Higher } \\
\text { concentration; Sweet, } \\
\text { fruity }\end{array}$ \\
\hline
\end{tabular}




\begin{tabular}{|c|c|c|c|c|}
\hline 99 & $\begin{array}{l}\text { 2-methylpropyl butanoate o } \\
\text { isobutyl butyrate }\end{array}$ & $539-90-2$ & $\begin{array}{l}\text { (1) n.q.; (5) } 0,54(\mathrm{ppm}) ;(\mathbf{6}) 4-34(\text { rel. to } \\
\text { 100ng) } ; \text {; (10) } 0,9-1,4(\mathrm{pA})^{\mathbf{h}}\end{array}$ & Fruity, ethereal \\
\hline 100 & 2-pentylfuran & $3777-69-3$ & (1) n.q. & \\
\hline 101 & 3-(methylthio)-1-propanol & $505-10-2$ & (3) n.q.; (4) 4,8( $\mu \mathrm{g} / \mathrm{kg})^{\mathbf{c}} ;$; (5) 1,25 (ppm) & \\
\hline 102 & 3-(methylthio)propanenitrile & $54974-63-9$ & (3) n.q. & \\
\hline 103 & 3-(methylthio)propyl acetate & $16630-55-0$ & $\begin{array}{l}\text { (1) n.q.; (2) n.q.; (3) n.q.; (4) 57,8( } \mu \mathrm{g} / \mathrm{kg})^{\text {c. }} \text {; (6) } \\
29(\text { rel. to 100ng) }\end{array}$ & \\
\hline 104 & 3,3-dimethyl-2-butanol & $464-07-3$ & (3) n.q. & \\
\hline 105 & 3-acetoxy-2-butanone & $4906-24-5$ & (4) $26,8(\mu \mathrm{g} / \mathrm{kg})^{\mathbf{c}}$ & \\
\hline 106 & 3-ethyl-2-methyl-1,3-hexadiene & $61142-36-7$ & (1) n.q. & \\
\hline 107 & 3-hexanol & $623-37-0$ & (3) n.q. & \\
\hline 108 & $\begin{array}{l}\text { 3-hydroxy-2,4,4- Trimethylpentyl } \\
\text { 2-methylpropanoate }\end{array}$ & $25265-77-4$ & (8) $1(\text { rel area })^{\mathrm{f}}$ & \\
\hline 109 & 3-hydroxy-2-butanone & $513-86-0$ & (3) n.q.; (4) $24(\mu \mathrm{g} / \mathrm{kg})^{\text {c. }}$; (5) n.d. & \\
\hline 110 & 3-methyl-1-butanol & $123-51-3$ & (1) n.q.; (5) 0,21 (ppm) & \\
\hline 111 & 3-methyl-2-butenyl acetate & $1191-16-8$ & (1) n.q.; (3) n.q.; (4) $12,9(\mu \mathrm{g} / \mathrm{kg})^{\mathrm{c}}$ & \\
\hline 112 & \begin{tabular}{|l|l|} 
3-methylbutanal o \\
isovaleraldehyde
\end{tabular} & $590-86-3$ & (11) $7,8(\mu \mathrm{g} / \mathrm{kg})^{\mathbf{i}}$ & \\
\hline 113 & $\begin{array}{l}\text { 3-methylbutyl acetate } o \text { isoamyl } \\
\text { acetate }\end{array}$ & $123-92-2$ & $\begin{array}{l}\text { (1) } 563(\mathrm{area})^{\mathbf{b}} ; \text {; (2) n.q.; (3) n.q.; (4) } \\
334,9(\mu \mathrm{g} / \mathrm{kg})^{\mathrm{c}} ; \text {; (5) n.d.; (10) 13-269,9(pA) }\end{array}$ & Fruity, sweet, banana \\
\hline 114 & \begin{tabular}{|l|} 
3-methylbutyl hexanoate o \\
Isoamyl hexanoate
\end{tabular} & 2198-61-0 & (1) n.q.; (2) n.q. & \\
\hline 115 & 3-methylpentane & $96-14-0$ & (8) 2,2(rel area) $)^{\mathrm{f}}$ & \\
\hline 116 & 3-nonenoic acid (tentative) & $4124-88-3$ & (9) $0,4(\% \text { area })^{\mathbf{g}}$ & \\
\hline 117 & 3-octanone & $106-68-3$ & (3) n.q. & \\
\hline 118 & $\begin{array}{l}\text { 4,7,7-Trimethylbicyclo[3.1.1]-3- } \\
\text { heptene }\end{array}$ & not found & (8) $0,3(\text { rel area })^{\mathbf{f}}$ & \\
\hline 119 & 4-heptanol & $589-55-9$ & (4) $5,7(\mu \mathrm{g} / \mathrm{kg})^{\mathbf{c}}$ & \\
\hline 120 & 4-oxononanal & $74327-29-0$ & (9) $0,3(\% \text { area })^{\mathbf{g}}$ & \\
\hline 121 & $\begin{array}{l}\text { 5,5,6-trimethyl-, endobicyclo[ } \\
\text { 2.2.1] -2-heptanone }\end{array}$ & $3292-05-05$ & (8) $0,5(\mathrm{rel} \text { area })^{\mathrm{f}}$ & \\
\hline 122 & 6-methyl-3-heptanone & $624-42-0$ & (3) n.q. & \\
\hline 123 & 6-methyl-5-hepten-2-one & $110-93-0$ & $\begin{array}{l}\text { (3) n.q.; (4) } 2(\mu \mathrm{g} / \mathrm{kg})^{\text {c }} ; \text { (8) } 0,9(\text { rel area) } \\
7,9-44(\mu \mathrm{f} / \mathrm{kg})^{\mathrm{i}}\end{array}$ & \\
\hline 124 & Acetaldehyde & 75-07-0 & (1) n.q.; (7) 0,1-0,7(\%A rel. $)^{\mathrm{e}}$ & \\
\hline 125 & Acetone & $67-64-1$ & (7) $0,1-2,3(\% \mathrm{~A} \text { rel. })^{\mathbf{e}}$ & \\
\hline 126 & Acetovanillone & $498-02-2$ & (3) n.q. & \\
\hline 127 & Allyl pentanoate & $6321-45-5$ & (3) n.q. & \\
\hline 128 & Amyl acetate $o$ pentyl acetate & $628-63-7$ & $\begin{array}{l}\text { (1) n.q.; (3) n.q.; (5) n.d.; (6) 20-46(rel. to } \\
\text { 100ng) } ; \text {; (10) 1,2-12,5(pA) }\end{array}$ & Banana, ethereal \\
\hline 129 & Anisaldehyde & $123-11-5$ & (3) n.q. & \\
\hline 130 & Benzaldehyde & $100-52-7$ & $\begin{array}{l}\text { (1) n.q.; (2) n.q.; (3) n.q.; (4) } 11,1(\mu \mathrm{g} / \mathrm{kg})^{\mathbf{c}} ; \mathbf{( 5 )} \\
0,75(\mathrm{ppm}) ;(\mathbf{6}) 3-10(\text { rel. to } 100 \mathrm{ng})^{\mathbf{d}} ;(\mathbf{8}) 11,2(\text { rel } \\
\text { area })^{\mathbf{f}} ; \mathbf{( 9 )} 0,4(\% \text { area) })^{\mathbf{g}} ;(\mathbf{1 0}) 1,2-1,5(\mathrm{pA})^{\mathbf{h}} ; \mathbf{( 1 1 )} \\
26,8-34,7(\mu \mathrm{g} / \mathrm{kg})^{\mathbf{i}}\end{array}$ & Aromatic, sweet \\
\hline 131 & $\begin{array}{l}\text { Benzyl acetate o phenylmethyl } \\
\text { acetate }\end{array}$ & $140-11-4$ & $\begin{array}{l}\text { (1) 5384884(area })^{\mathbf{b}} ;(\text { (2) n.q.; (3) n.q.; (4) } \\
94,3(\mu \mathrm{g} / \mathrm{kg})^{\mathbf{c}} ; \mathbf{( 5 )} 0,23(\mathrm{ppm}) ;(\mathbf{6}) 78-336(\text { rel. to } \\
\text { 100ng })^{\mathbf{d}} ;(\mathbf{7}) 0,9-5,9(\% \text { A rel. })^{\mathbf{e}} ;(\mathbf{9}) 0,4(\% \text { area })^{\mathbf{g}} ; \\
\text { (10) } 5,4-60,5(\mathrm{pA})^{\mathbf{h}}\end{array}$ & $\begin{array}{l}\text { Higher concentration; } \\
\text { Sweet, fruity }\end{array}$ \\
\hline 132 & Benzyl alcohol & $100-51-6$ & $\begin{array}{l}\text { (1) n.q.; (2) n.q.; (3) n.q.; (4) } 25,5(\mu \mathrm{g} / \mathrm{kg})^{\text {c }} ; \text { (5) } \\
5,21(\mathrm{ppm}) ;(\mathbf{9}) 7,27(\% \text { area) })^{\mathrm{g}} ;(\mathbf{1 1}) 23,4- \\
25,5(\mu \mathrm{g} / \mathrm{kg})^{\mathrm{i}}\end{array}$ & Floral, fruity \\
\hline 133 & Benzyl cyanide & $140-29-4$ & (3) n.q. & \\
\hline
\end{tabular}


Capítulo 1

\begin{tabular}{|c|c|c|c|c|}
\hline 134 & Butanal & $68648-78-2$ & (11) $\operatorname{tr} .(\mu \mathrm{g} / \mathrm{kg})^{\mathbf{i}}$ & \\
\hline 135 & $\begin{array}{l}\text { Butane-2,3-diol monoacetate o 3- } \\
\text { acetoxy-2-butanol }\end{array}$ & $56255-48-2$ & (3) n.q.; (4) $629(\mu \mathrm{g} / \mathrm{kg})^{\mathrm{c}}$ & \\
\hline 136 & Butanoic acid & $107-92-6$ & (3) n.q. & \\
\hline 137 & Butyl 1-acetate & $123-86-4$ & $\begin{array}{l}\text { (1) n.q.; (2) n.q.; (3) n.q.; (4)110,5( } \mu \mathrm{g} / \mathrm{kg})^{\mathbf{c}} ; \mathbf{( 5 )} \\
0,38(\mathrm{ppm}) ;(\mathbf{6}) 42-232(\text { rel. to } 100 \mathrm{ng})^{\mathbf{d}} ; \mathbf{( 8 )} \\
0,3(\text { rel area) }\end{array}$ & $\begin{array}{l}\text { Higher concentration; } \\
\text { Fruity }\end{array}$ \\
\hline 138 & Butyl butanoate & $109-21-7$ & (1) n.q.; (2) n.q.; (5) n.d.; (6) 2-4(rel. to 100ng) ${ }^{\mathbf{d}}$ & \\
\hline 139 & Butyl hexanoate & $626-82-4$ & (1) n.q. & \\
\hline 140 & Butyl propanoate & $590-01-2$ & (1) n.q.; (5) n.d.; (6) 4-12(rel. to 100ng) ${ }^{\mathbf{d}}$ & \\
\hline 141 & Carbon disulphide & $75-15-0$ & (11) $\operatorname{tr} .(\mu \mathrm{g} / \mathrm{kg})^{\mathbf{i}}$ & \\
\hline 142 & Chavicol & $501-92-8$ & (4) $83,6(\mu \mathrm{g} / \mathrm{kg})^{\mathbf{c}}$ & \\
\hline 143 & Cinnamyl acetate & $103-54-8$ & $(4) 71,1(\mu \mathrm{g} / \mathrm{kg})^{\mathbf{c}}$ & \\
\hline 144 & Cinnamyl alcohol & $104-54-1$ & (3) n.q.; (4) $125,5(\mu \mathrm{g} / \mathrm{kg})^{\mathbf{c}}$ & \\
\hline 145 & Decanal & $112-31-2$ & $\begin{array}{l}\text { (1) n.q.; (3) n.q.; (7) 0,2-1,1(\%A rel. })^{\text {e; }} ;(\mathbf{8}) \\
0,7(\text { rel area) })^{\text {f }} ; \text { (9) } \text { tr. }^{\mathrm{g}}\end{array}$ & \\
\hline 146 & Dihydroactinidiolide & $17092-92-1$ & (3) n.q. & \\
\hline 147 & \begin{tabular}{|l|l|}
$\begin{array}{l}\text { Dimethyl disulfide o } \\
\text { methyldisulfanylmethane }\end{array}$ \\
\end{tabular} & $624-92-0$ & (3) n.q.; (7) 0,2-2,7(\%A rel.. $)^{\mathbf{e}}$ & \\
\hline 148 & Dimethyl malonate & $108-59-8$ & (3) n.q. & \\
\hline 149 & Dimethyl sulfide & $75-18-3$ & (11) $\operatorname{tr} .(\mu \mathrm{g} / \mathrm{kg})^{\mathbf{i}}$ & \\
\hline 150 & Dimethyl trisulfide & $3658-80-8$ & (9) $\operatorname{tr}^{\mathrm{g}}$ & Cabbage \\
\hline 151 & $\begin{array}{l}\text { D-Limonene o 1-Methyl-4-prop-1- } \\
\text { en- 2-yl-cyclohexene }\end{array}$ & $5989-27-5$ & $\begin{array}{l}\text { (1) n.q.; (3) n.q.; (6) 2-11(rel. to 100ng) d; (8) } \\
0,4(\text { rel area) })^{\text {f }} \text { (11) } 21,6-29,5(\mu \mathrm{g} / \mathrm{kg})^{\mathrm{i}}\end{array}$ & \\
\hline 152 & Dodecane & $112-40-3$ & (1) n.q. & \\
\hline 153 & Dodecanoic acid & $143-07-7$ & (3) n.q. & \\
\hline 154 & Ethane-1,2-diol diacetate & not found & (3) n.q. & \\
\hline 155 & Ethanol & $64-17-5$ & $\begin{array}{l}\text { (1) n.q.; (6) 5-10(rel. to 100ng) })^{\text {di }} \text { (7) } 0,1-0,3(\% \mathrm{~A} \\
\text { rel. })^{\text {e }} \text {; (8) } 6,4(\text { (rel area) })^{\text {f }} \text {; (11) } 64,2-172(\mu \mathrm{g} / \mathrm{kg})^{\mathrm{i}}\end{array}$ & Ethanol \\
\hline 156 & Ethyl (E or Z)-4-heptenoate & $54340-70-4$ & $\begin{array}{l}\text { (1) n.q.; (2) n.q.; (6) tr.-22(rel. to 100ng) })^{\text {d; }} \text { (10) } \\
\text { 1,5(pA) }\end{array}$ & \\
\hline 157 & Ethyl (E)-2-butenoate & $623-70-1$ & (3) n.q. & \\
\hline 158 & Ethyl (E,E)-2,4-hexadienoate & $2396-84-1$ & (3) n.q. & \\
\hline 159 & \begin{tabular}{|l|}
$\begin{array}{l}\text { Ethyl 2-(methylthio)acetate o ethyl } \\
\text { (methylthio)acetate }\end{array}$ \\
\end{tabular} & $4455-13-4$ & $\begin{array}{l}\text { (1) n.q.; (2) n.q.; (3) n.q.; (4) 652,0( } \mu \mathrm{g} / \mathrm{kg})^{\mathbf{c}} ; \text { (5) } \\
0,29(\mathrm{ppm})\end{array}$ & \\
\hline 160 & Ethyl (Z)-4-octenoate & $34495-71-1$ & (1) n.q. & \\
\hline 161 & Ethyl 2-methylbutanoate & $7452-79-1$ & $\begin{array}{l}\text { (1) } 13975977(\text { area })^{\mathbf{b}} ;(\mathbf{2}) \text { n.q.; (3) n.q.; (4) } \\
\text { 98,3( } \mu \mathrm{gg} / \mathrm{kg})^{\mathbf{c}} ;(\mathbf{5}) 0,50(\mathrm{ppm}) ;(\mathbf{6}) 13-21(\text { rel. to } \\
100 \mathrm{ng})^{\mathbf{d}} ;(\mathbf{7}) 0,2-3,1(\% \text { A rel. })^{\mathbf{e}} ;(\mathbf{1 0}) 8,4- \\
\left.159,8(\mathrm{pA})^{\mathbf{h}} ;(\mathbf{1 1}) \text { tr.-114( } \mu \mathrm{g} / \mathrm{kg}\right)^{\mathbf{i}}\end{array}$ & $\begin{array}{l}\text { Cantaloupelike, floral, } \\
\text { fruity, melon; High aroma } \\
\text { value; Green, fruity }\end{array}$ \\
\hline 162 & $\begin{array}{l}\text { Ethyl 2-methylpropanoate o ethyl } \\
\text { isobutyrate }\end{array}$ & $97-62-1$ & 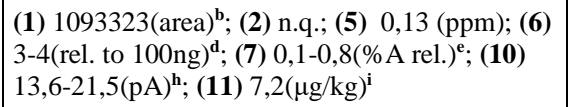 & $\begin{array}{l}\text { Foral, fruity, sweet; Main } \\
\text { melon aroma; Fruity, } \\
\text { floral }\end{array}$ \\
\hline 163 & Ethyl 3-(methylthio)propanoate & $13327-56-5$ & $\begin{array}{l}\text { (1) n.q.; (2) n.q.; (3) n.q.; (4) } 97,1(\mu \mathrm{g} / \mathrm{kg})^{\mathrm{c}} ; \text { (5) } \\
1,08 \text { (ppm) }\end{array}$ & Clean, fresh, melon, green \\
\hline 164 & \begin{tabular}{|l|}
$\begin{array}{l}\text { Ethyl 3-acetoxy-2- } \\
\text { methylbutanoate }\end{array}$ \\
\end{tabular} & $139564-43-5$ & (4) $22,3(\mu \mathrm{g} / \mathrm{kg})^{\mathbf{c}}$ & \\
\hline 165 & Ethyl 3-acetoxyhexanoate & $21188-61-4$ & (1) n.q. & \\
\hline 166 & Ethyl 3-hydroxybutanoate & $5405-41-4$ & (4) $15,7(\mu \mathrm{g} / \mathrm{kg})^{\mathrm{c}}$ & \\
\hline
\end{tabular}




\begin{tabular}{|c|c|c|c|c|}
\hline 167 & Ethyl 3-hydroxyhexanoate & $2305-25-1$ & (3) n.q. & \\
\hline 168 & $\begin{array}{l}\text { Ethyl 3-methylbutanoate o ethyl } \\
\text { isovalerate }\end{array}$ & $108-64-5$ & (3) n.q.; (11) $5,5(\mu \mathrm{g} / \mathrm{kg})^{\mathrm{i}}$ & \\
\hline 169 & Ethyl 3-phenylpropionate & $2021-28-5$ & (5) 0,48 (ppm) & \\
\hline 170 & Ethyl acetate & $141-78-6$ & 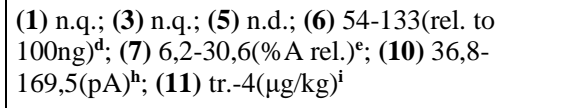 & Pineapple, ethereal \\
\hline 171 & Ethyl benzoate & $93-89-0$ & (1) n.q.; (3) n.q.; (10) $<0,3-4,4(\mathrm{pA})^{\mathbf{h}}$ & Floral, fruity \\
\hline 172 & Ethyl butanoate & $105-54-4$ & 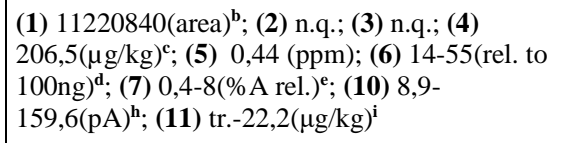 & $\begin{array}{l}\text { Sweet, fruity, candy; } \\
\text { Fruity, sweet }\end{array}$ \\
\hline 173 & Ethyl decanoate o ethyl caprate & $110-38-3$ & (1) n.q.; (10) $0,9-8,2(\mathrm{pA})^{\mathbf{h}} ;$ (11) $2,8(\mu \mathrm{g} / \mathrm{kg})^{\mathbf{i}}$ & Oily, fruity \\
\hline 174 & Ethyl dodecanoate o ethyl laurate & $106-33-2$ & (1) n.q.; (11) tr. $(\mu \mathrm{g} / \mathrm{kg})^{\mathrm{i}}$ & \\
\hline 175 & Ethyl ether & $60-29-7$ & (8) $4,6(\text { rel area })^{\mathbf{f}}$ & \\
\hline 176 & Ethyl heptanoate & $106-30-9$ & $\begin{array}{l}\text { (1) n.q.; (2) n.q.; (6) 2-10(rel. to } 100 \mathrm{ng})^{\mathbf{d}} ; \text {; (10) } \\
<0,3(\mathrm{pA})^{\mathbf{h}} ; \text {;11) tr. }(\mu \mathrm{g} / \mathrm{kg})^{\mathbf{i}}\end{array}$ & Winelike, fruity \\
\hline 177 & $\begin{array}{l}\text { Ethyl hexadecanoate o ethyl } \\
\text { palmitate }\end{array}$ & $628-97-7$ & (3) n.q. & \\
\hline 178 & Ethyl hexanoate & $123-66-0$ & $\begin{array}{l}\text { (1) } 1209671(\text { area })^{\mathbf{b}} ;(\mathbf{2}) \text { n.q.; (3) n.q.; (4) } \\
3,7(\mu \mathrm{g} / \mathrm{kg})^{\mathbf{c}} ; \mathbf{( 5 )} 0,05(\mathrm{ppm}) ;(\mathbf{6}) 10-45(\text { rel. to } \\
100 \mathrm{ng})^{\mathbf{d}} ;(\mathbf{1 0}) 297,4-2453,4(\mathrm{pA})^{\mathbf{h}} ;(\mathbf{1 1}) \\
25,7(\mu \mathrm{gg} / \mathrm{kg})^{\mathbf{i}}\end{array}$ & Fruity, apple \\
\hline 179 & Ethyl octanoate o ethyl caprylate & $106-32-1$ & (1) n.q.; (10) $1,8-9,7(\mathrm{pA})^{\mathbf{h}} ;$ (11) tr. $-4,9(\mu \mathrm{g} / \mathrm{kg})^{\mathbf{i}}$ & Fruity, floral \\
\hline 180 & Ethyl pentanoate o ethyl valerate & $539-82-2$ & $\begin{array}{l}\text { (1) n.q.; (2) n.q.; (4) } 13,6(\mu \mathrm{g} / \mathrm{kg})^{\mathrm{c}} ;(\mathbf{5}) \text { n.d.; (6) } 2- \\
\text { 9(rel. to } 100 \mathrm{ng})^{\mathbf{d}} ;(\mathbf{1 0}) 3,3-32,4(\mathrm{pA})^{\mathbf{h}} ;(\mathbf{1 1}) \\
\text { tr. }(\mu \mathrm{g} / \mathrm{kg})^{\mathbf{i}}\end{array}$ & Fruity, apple \\
\hline 181 & Ethyl phenyl acetate & $101-97-3$ & (1) n.q.; (2) n.q.; (3) n.q. & \\
\hline 182 & Ethyl propanoate & $105-37-3$ & $\begin{array}{l}\text { (1) n.q.; (2) n.q.; (3) n.q.; (5) } 0,45 \text { (ppm); (6) 8- } \\
12 \text { (rel. to 100ng) })^{\mathbf{d}} ;\left(\text { (11) } 2,3(\mu \mathrm{g} / \mathrm{kg})^{\mathbf{i}}\right.\end{array}$ & acid, butter (weak) \\
\hline 183 & Ethylbenzene & $100-41-4$ & (8) $0,4(\text { rel area })^{\mathbf{f}}$ & \\
\hline 184 & Ethylene 1,2-diacetate & $111-55-7$ & (9) tr. $^{\mathrm{g}}$ & \\
\hline 185 & Eugenol & $97-53-0$ & (3) n.q.; (4) $225,1(\mu \mathrm{g} / \mathrm{kg})^{\mathrm{c}}$ & \\
\hline 186 & Furfural & $98-01-1$ & (11) $9,3(\mu \mathrm{g} / \mathrm{kg})^{\mathbf{i}}$ & \\
\hline 187 & Geraniol & $106-24-1$ & (9) $0,2(\% \text { area })^{\mathrm{g}}$ & \\
\hline 188 & Geranyl acetone & $689-67-8$ & $\begin{array}{l}\text { (1) n.q.; (3) n.q.; (10) 3,1-12,5(pA) })^{\mathbf{h}} ; \text { (11) 4,4- } \\
6,6(\mu \mathrm{g} / \mathrm{kg})^{\mathbf{i}}\end{array}$ & Fresh, rosy \\
\hline 189 & Guaiacol & $90-05-1$ & (9) tr. $^{\mathrm{g}}$ & Phenolic \\
\hline 190 & Heliotropine o piperonal & $120-57-0$ & (3) n.q. & \\
\hline 191 & Heneicosane & $629-94-7$ & (9) $0,1(\% \text { area })^{\mathrm{g}}$ & \\
\hline 192 & Heptadecanal & $629-90-3$ & (1) n.q. & \\
\hline 193 & Heptadecane & $629-78-7$ & (1) n.q. & \\
\hline 194 & Heptadienyl acetate & $\begin{array}{c}\text { Tentative not } \\
\text { complete }\end{array}$ & (6) 7 (rel. to $100 \mathrm{ng})^{\mathbf{d}}$ & \\
\hline 195 & Heptanal & $111-71-7$ & $\begin{array}{l}\text { (1) n.q.; (4) } 4,4(\mu \mathrm{g} / \mathrm{kg})^{\mathbf{c}} ; \text { (8) } 2,7(\text { rel area) })^{\mathbf{f}} ; \text { (11) } \\
4,9-14,4(\mu \mathrm{g} / \mathrm{kg})^{\mathbf{i}}\end{array}$ & \\
\hline 196 & Heptyl acetate & $112-06-1$ & $\begin{array}{l}\text { (1) n.q.; (5) n.d.; (6) 36-64(rel. to 100ng) })^{\text {di }} \text {;(10) } \\
0,4-15,5(\mathrm{pA})^{\mathbf{h}}\end{array}$ & $\begin{array}{l}\text { Clean, fresh, flora; } \\
\text { Woody, oily }\end{array}$ \\
\hline 197 & Hexadecanal & $629-80-1$ & (1) n.q. & \\
\hline 198 & Hexadecane & $544-76-3$ & (1) n.q.; (9) 0,3(\%area) ${ }^{g}$ & \\
\hline
\end{tabular}




\begin{tabular}{|c|c|c|c|c|}
\hline 199 & Hexadecanoic acid o palmitic acid & $57-10-3$ & (1) n.q.; (3) n.q.; (9) 0,3(\%area) ${ }^{\mathrm{g}}$ & \\
\hline 200 & Hexanal & $66-25-1$ & $\begin{array}{l}\text { (1) } 116181(\text { area })^{\mathbf{b}} ; \text { (2) n.q.; (3) n.q.; (4) } \\
56,3(\mu \mathrm{g} / \mathrm{kg})^{\mathbf{c}} ;(\mathbf{5}) 0,25(\mathrm{ppm}) ;(\mathbf{6}) 5-7(\text { rel. to } \\
100 \mathrm{ng})^{\mathbf{d}} ; \mathbf{( 7 )} 0,4-51,8(\% \text { A rel. })^{\mathbf{e}} ;\left(\mathbf{( 8 )} 30(\text { rel area })^{\mathbf{f}} ;\right. \\
\text { (9) } 0,2(\% \text { area })^{\mathbf{g}} ;(\mathbf{1 1}) 50,4(\mu \mathrm{g} / \mathrm{kg})^{\mathbf{i}}\end{array}$ & Green \\
\hline 201 & Hexyl acetate & $142-92-7$ & 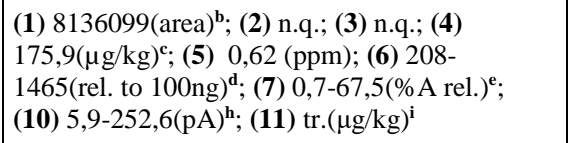 & $\begin{array}{l}\text { Higher concentration; } \\
\text { Apple, cherry }\end{array}$ \\
\hline 202 & Hexyl propanoate & $2445-76-3$ & (6) tr.-5(rel. to $100 \mathrm{ng})^{\text {d }}$ & \\
\hline 203 & Isoamyl butanoate & $106-27-4$ & (1) n.q.; (11) tr. $(\mu \mathrm{g} / \mathrm{kg})^{\mathrm{i}}$ & \\
\hline 204 & Isoamyl octanoate & $2035-99-6$ & (1) n.q. & \\
\hline 205 & Isoamyl propionate & $105-68-0$ & (5) n.d. & \\
\hline 206 & $\begin{array}{l}\text { Isobutyl 2-methylbutanoate o 2- } \\
\text { methylpropyl 2-methylbutanoate }\end{array}$ & $2445-67-2$ & (6) 4-23(rel. to 100ng) ; (11) tr. $(\mu \mathrm{g} / \mathrm{kg})^{\mathbf{i}}$ & \\
\hline 207 & $\begin{array}{l}\text { Isobutyl 2-methylpropanoate o } \\
\text { isobutyl isobutyrate }\end{array}$ & $97-85-8$ & (6) 5-25(rel. to 100ng) $)^{\mathbf{d}}$; (10) $1,2-2,9(\mathrm{pA})^{\mathbf{h}}$ & Ethereal, fruity \\
\hline 208 & $\begin{array}{l}\text { Isobutyl hexanoate o isobutyl } \\
\text { caproate o 2-methylpropyl } \\
\text { hexanoate }\end{array}$ & $105-79-3$ & (6) 4(rel. to $100 \mathrm{ng})^{\mathrm{d}}$ & \\
\hline 209 & Isobutyl propanoate & $540-42-1$ & (1) n.q.; (5) n.d.; (6) 11-31(rel. to 100ng) ${ }^{\mathbf{d}}$ & \\
\hline 210 & Isoeugenol & $97-54-1$ & (4) $2,7(\mu \mathrm{g} / \mathrm{kg})^{\mathbf{c}}$ & \\
\hline 211 & $\begin{array}{l}\text { Isopropyl acetate o propan-2-yl } \\
\text { acetate }\end{array}$ & $108-21-4$ & (1) n.q.; (7) $0,1-1(\% \text { A rel. })^{\mathrm{e}}$ & \\
\hline 212 & Isopropyl butanoate & $638-11-9$ & (1) n.q.; (6) 3-4(rel. to $100 \mathrm{ng})^{\mathbf{d}}$ & \\
\hline 213 & Linalool & $78-40-6$ & (11) $\operatorname{tr} .(\mu \mathrm{g} / \mathrm{kg})^{\mathrm{i}}$ & \\
\hline 214 & Methanethiol & 74-93-1 & (7) $0,1-5,2(\% \text { A rel. })^{\mathrm{e}} ;(\mathbf{8}) 10,6(\mathrm{rel} \text { area })^{\mathrm{f}}$ & \\
\hline 215 & Methoxyeugenol & $93-15-2$ & (4) $21,4(\mu \mathrm{g} / \mathrm{kg})^{\mathbf{c}}$ & \\
\hline 216 & $\begin{array}{l}\text { Methyl (methylthio)acetate o } \\
\text { methyl 2-(methylthio)acetate }\end{array}$ & $16630-66-3$ & (3) n.q.; (4) $25,8(\mu \mathrm{g} / \mathrm{kg})^{\mathbf{c}} ;$ (5) 0,75 (ppm) & $\begin{array}{l}\text { Baked potato, pungent, } \\
\text { rancid }\end{array}$ \\
\hline 217 & Methyl 2-methylbutanoate & $868-57-5$ & 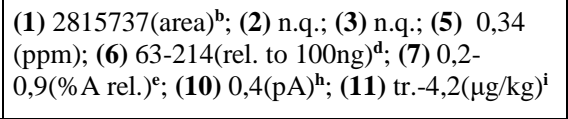 & $\begin{array}{l}\text { Artificial strawberry, } \\
\text { fruity, sweet; High aroma } \\
\text { value; Fruity, sweet }\end{array}$ \\
\hline 218 & $\begin{array}{l}\text { Methyl 2-methylpropanoate o } \\
\text { methyl isobutyrate }\end{array}$ & $547-63-7$ & $\begin{array}{l}\text { (1) n.q.; (2) n.q.; (5) n.d.; (6) tr.-13(rel. to } \\
\text { 100ng) })^{\mathbf{d}}\end{array}$ & \\
\hline 219 & $\begin{array}{l}\text { Methyl 2-sulfanylacetate o methyl } \\
\text { mercaptoacetate }\end{array}$ & $64807-90-5$ & (7) $0,1-2,1(\% \mathrm{~A} \mathrm{rel} .)^{\mathrm{e}}$ & \\
\hline 220 & Methyl 3-(methylthio)propanoate & $13532-18-8$ & (1) n.q.; (2) n.q.; (3) n.q. & \\
\hline 221 & Methyl 3-phenylpropionate & $103-25-3$ & (5) 0,47 (ppm) & \\
\hline 222 & Methyl acetate & 79-20-9 & $\begin{array}{l}\text { (1) n.q.; (3) n.q.; (6) 8-61(rel. to 100ng) })^{\text {d; }} \text { (7) } \\
0,6-1,2(\% \text { A rel. })^{\text {e }}\end{array}$ & \\
\hline 223 & Methyl butanoate & $623-42-7$ & $\begin{array}{l}\text { (1) n.q.; (3) n.q.; (5) } 0,50 \text { (ppm); (6) 4-50(rel. to } \\
\text { 100ng) })^{\mathbf{d}} ;(\mathbf{7}) 0,3-1(\% \text { A rel. })^{\text {e }} ; \text { (10) } 16,4- \\
17,2(\mathrm{pA})^{\mathbf{h}}\end{array}$ & Fruity \\
\hline 224 & $\begin{array}{l}\text { Methyl diethyl carbamodithioic } \\
\text { acid }\end{array}$ & $686-07-7$ & (1) n.q. & \\
\hline 225 & \begin{tabular}{|l|} 
Methyl dodecanoate o methyl \\
laurate
\end{tabular} & $111-82-0$ & (11) $\operatorname{tr} .(\mu \mathrm{g} / \mathrm{kg})^{\mathrm{i}}$ & \\
\hline
\end{tabular}




\begin{tabular}{|c|c|c|c|c|}
\hline 226 & Methyl heptanoate & $106-73-0$ & (1) n.q.; (2) n.q.; (6) 3-13(rel. to 100ng) ${ }^{\mathbf{d}}$ & \\
\hline 227 & Methyl hexanoate & $106-70-7$ & $\begin{array}{l}\text { (1) n.q.; (2) n.q.; (3) n.q.; (6) 2-33(rel. to } \\
\text { 100ng)d ; (10) 0,4-12,3(pA) }\end{array}$ & Pineapple, ethereal \\
\hline 228 & $\begin{array}{l}\text { Methyl octanoate o methyl } \\
\text { caprylate }\end{array}$ & $111-11-5$ & (6) $1-8$ (rel. to $100 \mathrm{ng})^{\mathbf{d}}$ & \\
\hline 229 & $\begin{array}{l}\text { Methyl pentanoate o methyl } \\
\text { valerate }\end{array}$ & $624-24-8$ & (1) n.q.; (6) tr.-6(rel. to $100 \mathrm{ng})^{\mathbf{d}}$ & \\
\hline 230 & Methyl propanoate & $554-12-1$ & $\begin{array}{l}\text { (1) n.q.; (5) n.d.; (6) 3-18(rel. to 100ng) })^{\text {d. }} \text { (7) } \\
0,1-0,3(\% \text { A rel. })^{\mathbf{e}}\end{array}$ & \\
\hline 231 & Methylcyclopentane & $96-37-7$ & (8) $2,5(\text { rel area })^{\text {f }}$ & \\
\hline 232 & Nonadecane & $629-92-5$ & (1) n.q. & \\
\hline 233 & Nonanal & $124-19-6$ & $\begin{array}{l}\text { (1) } 61102(\text { area })^{\mathbf{b}} ;(\mathbf{2}) \text { n.q.; (3) n.q.; (6) 5-133(rel. } \\
\text { to } 100 \mathrm{ng})^{\mathbf{d}} ; \mathbf{( 7 )} 0,3-2,6(\% \text { A rel. })^{\mathbf{e}} ;(\mathbf{9}) \\
3,9(\% \text { area })^{\mathbf{g}} ;(\mathbf{1 0}) 9,1-26,8(\mathrm{pA})^{\mathbf{h}} ;(\mathbf{1 1}) 33,6- \\
107(\mu \mathrm{g} / \mathrm{kg})^{\mathbf{i}}\end{array}$ & Cilantro leaf; Fatty, melon \\
\hline 234 & Nonanoic acid & $112-05-0$ & (9) $0,8(\% \text { area })^{\mathbf{g}}$ & \\
\hline 235 & Nonyl acetate & $143-13-5$ & $\begin{array}{l}\text { (3) n.q.; (6) 14-28(rel. to 100ng)d; (10) 1,8- } \\
15(\mathrm{pA})^{\mathrm{h}}\end{array}$ & Fruity \\
\hline 236 & Octadecanal & $638-66-4$ & (1) n.q. & \\
\hline 237 & Octadecane & $593-45-3$ & (1) n.q.; (9) 0,5(\%area) $)^{g}$ & \\
\hline 238 & Octadecanoic acid o stearic acid & $57-11-4$ & (1) n.q.; (3) n.q. & \\
\hline 239 & Octanal & $124-13-0$ & $\begin{array}{l}\text { (1) n.q.; (2) n.q.; (4) } 46,8(\mu \mathrm{g} / \mathrm{kg})^{\mathbf{c}} ;(\mathbf{( 8 )}) 1,8(\mathrm{rel} \\
\text { area); ; (9) n.q.; (11) } 5,4-19,8(\mu \mathrm{g} / \mathrm{kg})^{\mathbf{i}}\end{array}$ & Fresh \\
\hline 240 & Octyl acetate & $112-14-1$ & $\begin{array}{l}\text { (1) n.q.; (5) } 0,93(\mathrm{ppm}) ;(\mathbf{6}) 9-33 \text { (rel. to } \\
\text { 100ng) } ; \text {; (10) } 4-11,1(\mathrm{pA})^{\mathbf{h}}\end{array}$ & Fruity, floral \\
\hline 241 & p-cymene & $99-87-6$ & (6) $1-4$ (rel. to $100 \mathrm{ng})^{\mathbf{d}}$ & \\
\hline 242 & Pentadecan-1-ol & $629-76-5$ & (9) $0,1(\% \text { area })^{\mathrm{g}}$ & \\
\hline 243 & Pentadecanal & 2765-11-09 & (1) n.q. & \\
\hline 244 & Pentadecane & $629-62-9$ & (1) n.q. & \\
\hline 245 & Pentanal o valeraldehyde & $110-62-3$ & (1) n.q.; (7) 0,1-7(\%A rel. $)^{\mathbf{e}} ;$ (8) 2,6(rel area $)^{\mathbf{f}}$ & \\
\hline 246 & Pentyl hexanoate o amyl caproate & $540-07-8$ & (1) n.q.; (2) n.q. & \\
\hline 247 & $\begin{array}{l}\text { Pentyl propanoate o amyl } \\
\text { propionate }\end{array}$ & $624-54-4$ & (1) n.q. & \\
\hline 248 & $\begin{array}{l}\text { Phenethyl acetate o 2-phenylethyl } \\
\text { acetate }\end{array}$ & $103-45-7$ & $\begin{array}{l}\text { (1) n.q.; (2) n.q.; (3) n.q.; (4) } 14,6(\mu \mathrm{g} / \mathrm{kg})^{\mathrm{c}} ; \text { (5) } \\
0,25(\mathrm{ppm}) ;(\mathbf{6}) 2-72(\text { rel. to } 100 \mathrm{ng})^{\mathrm{d}} ;(\mathbf{1 0}) 3,6- \\
7,3(\mathrm{pA})^{\mathbf{h}}\end{array}$ & \\
\hline 249 & $\begin{array}{l}\text { Phenyl ethyl alcohol o 2- } \\
\text { phenylethanol }\end{array}$ & $60-12-8$ & $\begin{array}{l}\text { (1) n.q.; (2) n.q.; (3) n.q.; (4) 7,3( }(\mu \mathrm{g} / \mathrm{kg})^{\mathbf{c}} ; \text { (5) } \\
1,79(\mathrm{ppm}) ; \text {;9) n.q.; (11) } 12(\mu \mathrm{g} / \mathrm{kg})^{\mathbf{i}}\end{array}$ & $\begin{array}{l}\text { Sweet, floral; High aroma } \\
\text { value }\end{array}$ \\
\hline 250 & $\begin{array}{l}\text { Phenyl propyl acetate o 3-phenyl } \\
\text { propyl acetate }\end{array}$ & $122-72-5$ & (1) n.q.; (3) n.q.; (4) $123,9(\mu \mathrm{g} / \mathrm{kg})^{\mathbf{c}}$ & \\
\hline 251 & $\begin{array}{l}\text { Phenyl propyl alcohol o 3-Phenyl- } \\
\text { 1-propanol o Benzenepropanol }\end{array}$ & $122-97-4$ & $\begin{array}{l}\text { (1) n.q.; (2) n.q.; (3) n.q.; (4) 216,5( } \mathrm{\mu g} / \mathrm{kg})^{\mathrm{c}} ; \text {; (5) } \\
3,1 \text { (ppm); (9) 0,1(\%area) }\end{array}$ & \\
\hline 252 & $\begin{array}{l}\text { Phenylacetaldehyde o } \\
\text { Benzeneacetaldehyde o 2- } \\
\text { phenylacetaldehyde }\end{array}$ & $122-78-1$ & $\begin{array}{l}\text { (1) n.q.; (2) n.q.; (3) n.q.; (7) 0,3-3(\%A rel.) })^{\text {e }} \text {; (9) } \\
0,2(\% \text { area) })^{\mathrm{g}}\end{array}$ & Sweet, floral \\
\hline 253 & Pinocarvone & $30460-92-5$ & (9) $0,1(\% \text { area })^{\mathrm{g}}$ & \\
\hline 254 & p-menth-1-en-8-ol o $\alpha$-terpineol & $98-55-5$ & (3) n.q.; (4) $9,4(\mu \mathrm{g} / \mathrm{kg})^{\mathbf{c}}$ & \\
\hline 255 & p-methylanisole o 4-methylanisole & $104-93-8$ & (1) n.q. & \\
\hline 256 & Propanal & $123-38-6$ & (1) n.q.; (3) n.q.; (11) tr. $(\mu \mathrm{g} / \mathrm{kg})^{\mathbf{i}}$ & \\
\hline 257 & Propane-1,2-diol diacetate & $623-84-7$ & (3) n.q. & \\
\hline
\end{tabular}




\begin{tabular}{|c|c|c|c|c|}
\hline 258 & Propyl 2-methylbutanoate & $37064-20-3$ & (1) n.q.; (11) tr. $(\mu \mathrm{g} / \mathrm{kg})^{\mathbf{i}}$ & \\
\hline 259 & Propyl acetate & $109-60-4$ & $\begin{array}{l}\text { (1) n.q.; (3) n.q.; (5) 0,08 (ppm); (6) 9-27(rel. to } \\
\text { 100ng) }{ }^{\mathbf{d}} ; \text { (7) } 0,5-3,2(\% \text { A rel. })^{\mathbf{e}}\end{array}$ & \\
\hline 260 & Propyl butanoate & $105-66-8$ & (1) n.q.; (3) n.q.; (5) n.d.; (6) 1-4(rel. to 100ng) ${ }^{\text {d }}$ & \\
\hline 261 & Propyl hexanoate & $626-77-7$ & (1) n.q.; (2) n.q. & \\
\hline 262 & \begin{tabular}{|l|}
$\begin{array}{l}\text { Propyl pentanoate o propyl } \\
\text { valerate }\end{array}$ \\
\end{tabular} & $141-06-0$ & (3) n.q. & \\
\hline 263 & Propyl propanoate & $106-36-5$ & (1) n.q.; (5) n.d.; (6) 2-4(rel. to 100ng) ${ }^{\mathbf{d}}$ & \\
\hline 264 & S-methyl 2-methylbutanethioate & $42075-45-6$ & (6) 5-75(rel. to 100ng) ${ }^{\mathrm{d}}$ & \\
\hline 265 & S-methyl 2-methylpropanethioate & $42075-42-3$ & (6) $2-16$ (rel. to $100 \mathrm{ng})^{\mathrm{d}}$ & \\
\hline 266 & S-methyl 3-methylbutanethioate & $23747-45-7$ & (1) n.q.; (2) n.q. & \\
\hline 267 & S-methyl ethanethioate & $1534-08-3$ & (1) n.q.; (6) 1-9(rel. to $100 \mathrm{ng})^{\mathbf{d}}$ & \\
\hline 268 & Squalene & $111-02-4$ & (9) $0,1(\% \text { area })^{\mathrm{g}}$ & \\
\hline 269 & Tetradecane & $629-59-4$ & (1) n.q. & \\
\hline 270 & Tetradecanoic acid o myristic acid & $544-63-8$ & (3) n.q. & \\
\hline 271 & Tetrahydro-5-pentyl-2-furanol & $78053-99-3$ & (9) $1,4(\% \text { area })^{\mathrm{g}}$ & \\
\hline 272 & Thymol & $89-83-8$ & (11) $\operatorname{tr} .(\mu \mathrm{g} / \mathrm{kg})^{\mathbf{i}}$ & \\
\hline 273 & trans-4,5-epoxy-(E)-2-decenal & $134454-31-2$ & (9) n.q. & Green, metallic \\
\hline 274 & Undecanal & $112-44-7$ & (1) n.q. & \\
\hline 275 & Vanillin & $121-33-5$ & (4) $2,1(\mu \mathrm{g} / \mathrm{kg})^{\mathbf{c}}$ & \\
\hline 276 & Verbenone & $80-57-9$ & (3) n.q. & \\
\hline 277 & a-farnesene & $502-61-4$ & (1) n.q.; (10) $<0,3(\mathrm{pA})^{\mathbf{h}}$ & \\
\hline 278 & $\alpha$-terpinyl acetate & $80-26-2$ & (3) n.q.; (6) tr.-6(rel. to 100ng) ${ }^{\mathbf{d}}$ & \\
\hline 279 & $\beta$-cyclocitral & $5392-40-5$ & (1) n.q.; (3) n.q.; (11) $11,1(\mu \mathrm{g} / \mathrm{kg})^{\mathbf{i}}$ & \\
\hline 280 & $\beta$-ionone & $14901-07-6$ & (1) n.q.; (3) n.q.; (11) $5,5(\mu \mathrm{g} / \mathrm{kg})^{\mathbf{i}}$ & \\
\hline 281 & $\beta$-pinene & $127-91-3$ & (3) n.q.; (11) tr. $(\mu \mathrm{g} / \mathrm{kg})^{\mathrm{i}}$ & \\
\hline 282 & $\gamma$-dodecalactone & 2305-05-07 & (4) $11,3(\mu \mathrm{g} / \mathrm{kg})^{\mathbf{c}}$ & \\
\hline 283 & $\gamma$-hexalactone & 695-06-7 & (4) $41,3(\mu \mathrm{g} / \mathrm{kg})^{\mathbf{c}}$ & \\
\hline 284 & $\gamma$-decalactone & $706-14-9$ & (3) n.q.; (4) $36,6(\mu \mathrm{g} / \mathrm{kg})^{\mathbf{c}}$ & \\
\hline 285 & $\gamma$-nonalactone & $104-61-0$ & (3) n.q. & \\
\hline 286 & $\gamma$-octalactone & $104-50-7$ & (3) n.q.; (4) $27,3(\mu \mathrm{g} / \mathrm{kg})^{\mathbf{c}}$ & \\
\hline 287 & $\gamma$-terpinene & $99-85-4$ & (3) n.q. & \\
\hline 288 & $\delta$-decalactone & $705-86-2$ & (4) $3,7(\mu \mathrm{g} / \mathrm{kg})^{\mathbf{c}}$ & \\
\hline 289 & $\delta$-nonalactone & $3301-94-8$ & (3) n.q. & \\
\hline 290 & $\delta$-octalactone & $698-76-0$ & (4) $1,7(\mu \mathrm{g} / \mathrm{kg})^{\mathbf{c}}$ & \\
\hline 291 & $\delta$-undecalactone & $710-04-3$ & (3) n.q. & \\
\hline
\end{tabular}

tr. $=$ traces.

n.d. $=$ not detected.

n.q. = not quantitate data was presented.

${ }^{a}=$ Chemical abstract service.

$\mathrm{b}=$ Recovery of each compound is based on MS target ions in optimal maturity stage.

${ }^{c}=\mu \mathrm{g} / \mathrm{kg}$ equivalent of 2 -octanol.

${ }^{\mathrm{d}}=$ Concentration relative to 1,2 dichlorobenzene $(100 \mathrm{ng})$ injected onto GC column.

$\mathrm{e}=$ Average of relative area percentage on a total peaks area basis.

$\mathrm{f}=$ Relative content expressed as percentage of total chromatographic area of the 28 volatile compounds of the profile reported.

$\mathrm{g}=$ Peak area $\%$ is determined from the mass spectrometer total ion chromatogram.

${ }^{\mathrm{h}}=$ Reported in detector response units of picoamperes (pA).

${ }^{\mathrm{i}}=\mu \mathrm{g} / \mathrm{kg}$ in single volatile compounds and classes of substances. 
(1) Beaulieu, J.C., Grimm, C.C., 2001. Identification of volatile compounds in cantaloupe at various developmental stages using solid phase microextraction. Journal of Agricultural and Food Chemistry, 49, 1345-1352.

(2) Beaulieu, J.C., Lancaster, V.A., 2007. Correlating volatile compounds, sensory attributes, and quality parameters in stored fresh-cut cantaloupe. Journal of Agricultural and Food Chemistry, 55, 9503-9513.

(3) Homatidou, V.I., Karvouni, S.S., Dourtoglou, V.G., Poulos, C.N., 1992. Determination of total volatile components of Cucumis melo L. variety Cantaloupensis. Journal of Agricultural and Food Chemistry, 40, 1385-1388.

(4) Aubert, C., Pitrat, M., 2006. Volatile compounds in the skin and pulp of Queen Anne's pocket melon. Journal of Agricultural and Food Chemistry, 54, 8177-82.

(5) Jordán, M.J., Shaw, P.E., Goodner, K.L., 2001. Volatile components in aqueous essence and fresh fruit of Cucumis melo cv. Athena (muskmelon) by GC-MS and GC-O. Journal of Agricultural and Food Chemistry, 49, 5929-5933.

(6) Kourkoutas, D., Elmore, J.S., Mottram, D.S., 2006. Comparison of the volatile compositions and flavour properties of Cantaloupe, Galia and Honeydew muskmelons. Food Chemistry, 97, 95-102.

(7) Obando-Ulloa, J.M., Moreno, E., 2008. Climacteric or non-climacteric behavior in melon fruit: 1. Aroma volatiles. Postharvest Biology and Technology, 49, 27-37.

(8) Obando-Ulloa, J.M., Ruiz, J., 2010. Aroma profile of a collection of near-isogenic lines of melon (Cucumis melo L.). Food Chemistry, 118, 815-822.

(9) Perry, P. L., Wang, Y., Lin, J., 2009. Analysis of Honeydew melon (Cucumis melo var. inodorus) flavour and GC-MS/MS identification of (E,Z)-2,6-nonadienyl acetate. Flavour and Fragrance Journal, 24, 341-347.

(10) Saftner, R., Abbott, J. A., Lester, G., Vinyard, B., 2006. Sensory and analytical comparison of orange-fleshed Honeydew to Cantaloupe and green-fleshed Honeydew for fresh-cut chunks. Postharvest Biology and Technology, 42, 150-160.

(11) Verzera, A., Dima, G., Tripodi, G., \& Ziino, M., 2011. Fast quantitative determination of aroma volatile constituents in melon fruits by headspace-solidphase microextraction and gas chromatography-mass spectrometry. Food Analytical Methods, 4, 141-149. 


\section{REFERENCES}

Amaro, A.L., Beaulieu, J.C., Grimm, C.C., Stein, R.E., Almeida, D.P.F., 2012. Effect of oxygen on aroma volatiles and quality of fresh-cut Cantaloupe and Honeydew melons. Food Chem. 130, 49-57.

Anderson, M.J., Whitcomb, P.J., 2005. RSM simplified. Optimizing processes using response surface methods for design of experiments. New York CRC Pres.

Aubert, C., Bourger, N., 2004. Investigation of volatiles in Charentais cantaloupe melons (Cucumis melo var. cantalupensis). Characterization of aroma constituents in some cultivars. J. Agric. Food Chem. 52, 4522-4528.

Aubert, C., Pitrat, M., 2006. Volatile compounds in the skin and pulp of Queen Anne's pocket melon. J. Agric. Food Chem. 54, 8177-82.

Beaulieu, J.C., 2006a. Effect of cutting and storage on acetate and nonacetate esters in convenient, ready-to-eat fresh-cut melons and apples. HortScience 41, 65-73.

Beaulieu, J.C., 2006b. Volatile changes in cantaloupe during growth, maturation, and in stored fresh-cuts prepared from fruit harvested at various maturities. J. Am. Soc. Hortic. Sci. 131, 127-139.

Beaulieu, J.C., 2005. Within-season volatile and quality differences in stored fresh-cut cantaloupe cultivars. J. Agric. Food Chem. 53, 8679-8687.

Beaulieu, J.C., Grimm, C.C., 2001. Identification of volatile compounds in cantaloupe at various developmental stages using solid phase microextraction. J. Agric. Food Chem. 49, 1345-1352.

Beaulieu, J.C., Ingram, D.A., Lea, J.M., Bett-Garner, K.L., 2004. Effect of harvest maturity on the sensory characteristics of fresh-cut cantaloupe. J. Food Sci. 69, S250-S258.

Beaulieu, J.C., Lancaster, V.A., 2007. Correlating volatile compounds, sensory attributes, and quality parameters in stored fresh-cut cantaloupe. J. Agric. Food Chem. 55, 9503-9513. 
Beaulieu, J.C., Lea, J.M., 2006. Characterization and semiquantitative analysis of volatiles in seedless watermelon varieties using solid-phase microextraction. J. Agric. Food Chem. 54, 7789-7793.

Beltran, J., Serrano, E., López, F.J., Peruga, A., Valcarcel, M., Rosello, S., 2006. Comparison of two quantitative GC-MS methods for analysis of tomato aroma based on purge-and-trap and on solid-phase microextraction. Anal. Bioanal. Chem. $385,1255-1264$.

Bicchi, C., Cordero, C., Liberto, E., Rubiolo, P., Sgorbini, B., 2004. Automated headspace solid-phase dynamic extraction to analyse the volatile fraction of food matrices. J. Chromatogr. A 1024, 217-226.

Buttery, R.G., Seifert, R.M., Ling, L.C., Soderstrom, E.L., Ogawa, J.M., Turnbaugh, J.G., 1982. Additional aroma components of Honeydew melon. J. Agric. Food Chem. 30, $1208-1211$.

Contarini, G., Povolo, M., 2002. Volatile fraction of milk: comparison between purge and trap and solid phase microextraction techniques. J. Agric. Food Chem. 50, 73507355.

Gonda, I., Bar, E., Portnoy, V., Lev, S., Burger, J., Schaffer, A.A., Tadmor, Y., Gepstein, S., Giovannoni, J.J., Katzir, N., Lewinsohn, E., 2010. Branched-chain and aromatic amino acid catabolism into aroma volatiles in Cucumis melo L. fruit. J. Exp. Bot. $61,1111-1123$.

Homatidou, V.I., Karvouni, S.S., Dourtoglou, V.G., Poulos, C.N., 1992. Determination of total volatile components of Cucumis melo L. variety Cantaloupensis. J. Agric. Food Chem. 40, 1385-1388.

Horvat, R., Senter, S.D., 1987. Identification of additional volatile compounds from cantaloupe. J. Food Sci. 52, 1097-1098.

Jordán, M.J., Shaw, P.E., Goodner, K.L., 2001. Volatile components in aqueous essence and fresh fruit of Cucumis melo cv. Athena (muskmelon) by GC-MS and GC-O. J. Agric. Food Chem. 49, 5929-5933.

Kemp, T.R., 1975. Identification of some volatile compounds from Citrullus vulgaris. Phytochemistry 14, 2637-2638. 
Kim, K.S., Lee, H.J., Kim, S.M., 1999. Volatile flavor components in watermelon (Citrullus vulgaris S.) and oriental melon (Cucumis melo L.). Korean J. Food Sci. $31,322-328$.

Kourkoutas, D., Elmore, J.S., Mottram, D.S., 2006. Comparison of the volatile compositions and flavour properties of Cantaloupe, Galia and Honeydew muskmelons. Food Chem. 97, 95-102.

Kovats, E., 1958. Gas-chromatographische charakterisierung organischer verbindungen. Teil 1: retentionsindices aliphatischer halogenide, alkohole, aldehyde und ketone. Helv. Chim. Acta 41, 1915-1932.

Kozłowska, K., Polkowska, Ż., Namieśnik, J., Przyjazny, A., 2006. Investigation of stability of aqueous solutions containing trace amounts of volatile organic analytes. TrAC Trends Anal. Chem. 25, 609-620.

Kultur, F., Harrison, H.C., Staub, J.E., 2001. Spacing and genotype affect fruit sugar concentration, yield, and fruit size of muskmelon. HortScience 36, 274-278.

Lamikanra, O., Richard, O.A., Parker, A., 2002. Ultraviolet induced stress response in fresh cut cantaloupe. Phytochemistry 60, 27-32.

Lester, G., Shellie, K.C., Ice, M., Dew, M., Cream, H., Brew, H., 1992. Postharvest sensory and physicochemical attributes of honey dew melon fruits. Hortscience 27, 1012-1014.

Lignou, S., Parker, J.K., Baxter, C., Mottram, D.S., 2014. Sensory and instrumental analysis of medium and long shelf-life Charentais cantaloupe melons (Cucumis melo L.) harvested at different maturities. Food Chem. 148, 218-229.

Liu, C., Zhang, H., Dai, Z., Liu, X., Liu, Y., Deng, X., Chen, F., Xu, J., 2012. Volatile chemical and carotenoid profiles in watermelons [Citrullus vulgaris (Thunb.) Schrad (Cucurbitaceae)] with different flesh colors. Food Sci. Biotechnol. 21, 531541.

Monforte, A.J., Alvarez, J.M., 2006. Mejora de la calidad del melón, in: Llácer, G., Díez, M.J., Carrillo, J.M., Badenes, M.L. (Eds.), Mejora genética de la calidad en plantas. Soc. Española de Ciencias Hortícolas; Soc. Española de Genética, Valencia, pp. 393-413. 
Obando-Ulloa, J.M., Moreno, E., García-Mas, J., Nicolai, B., Lammertyn, J., Monforte, A.J., Fernández-Trujillo, J.P., 2008. Climacteric or non-climacteric behavior in melon fruit: 1. Aroma volatiles. Postharvest Biol. Technol. 49, 27-37.

Obando-Ulloa, J.M., Ruiz, J., 2010. Aroma profile of a collection of near-isogenic lines of melon (Cucumis melo L.). Food Chem. 118, 815-822.

Perry, P.L., Wang, Y., Lin, J., 2009. Analysis of Honeydew melon (Cucumis melo var. inodorus) flavour and GC-MS/MS identification of (E,Z)-2,6-nonadienyl acetate. Flavour Fragr. J. 24, 341-347.

Saftner, R., Abbott, J.A., Lester, G., Vinyard, B., 2006. Sensory and analytical comparison of orange-fleshed Honeydew to Cantaloupe and green-fleshed Honeydew for freshcut chunks. Postharvest Biol. Technol. 42, 150-160.

Verzera, A., Dima, G., Tripodi, G., Ziino, M., 2011. Fast quantitative determination of aroma volatile constituents in melon fruits by headspace-solid-phase microextraction and gas chromatography-mass spectrometry. Food Anal. Methods 4, 141-149.

Wyllie, S.G., Leach, D.N., Wang, Y., Shewfelt, R.L., 1995. Key aroma compounds in melons. Their development and cultivar dependence, in: Rouseff, R.L., Leahy, M.M. (Eds.), Fruit flavors: biogenesis, characterization, and authentication. American Chemical Society: ACS Symposium Series 596, Washington, D.C., pp. $248-257$.

Yabumoto, K., Yamaguchi, M., Jennings, W.G., 1978. Production of volatile compounds by muskmelon, Cucumis melo. Food Chem. 3, 7-16.

Yajima, I., Sakakibara, H., Ide, J., Yanai, T., Hayashi, K., 1985. Volatile flavor components of watermelon (Citrullus vulgaris). Agric. Biol. Chem. 49, 3145-3150.

Yamaguchi, M., Hughes, D.L., Yabumoto, K., Jennings, W.G., 1977. Quality of cantaloupe muskmelons: variability and attributes. Sci. Hortic. 6, 59-70.

Yau, E.W., Rosnah, S., Noraziah, M., Chin, N.L., Osman, H., 2010. Physico-chemical compositions of the red seedless watermelons (Citrullus Lanatus). Int. Food Res. J. $17,327-334$. 


\section{CAPÍtULO 2}

"Fruit quality assessment of watermelons grafted onto citron melon rootstock"

Fredes A., Roselló S., Beltrán J., Cebolla-Cornejo J., Pérez-de-Castro A., Gisbert C., Picó M.B. (2017). Journal of the Science of Food and Agriculture, 97 (5), 1646-1655.

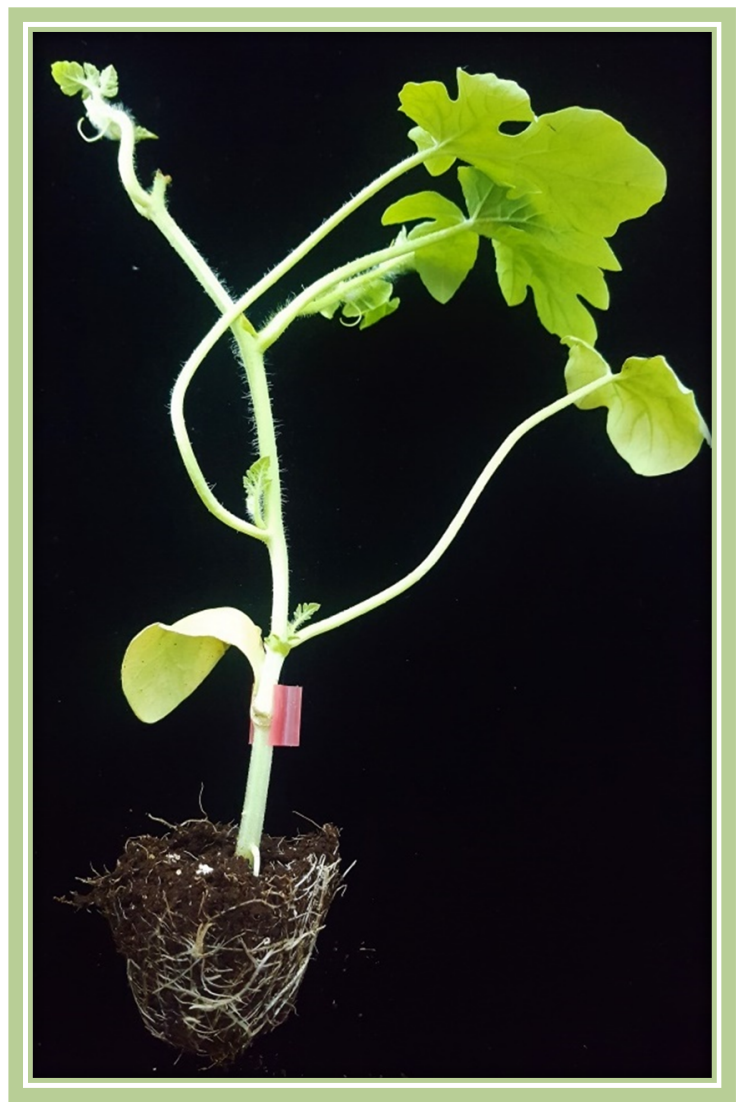





\title{
Fruit quality assessment of watermelons grafted onto citron melon rootstock
}

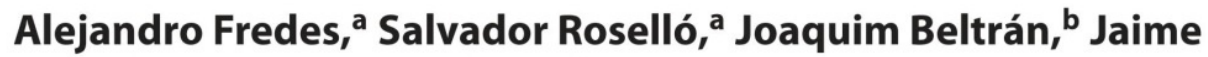 \\ Cebolla-Cornejo, ${ }^{c}$ Ana Pérez-de-Castro, ${ }^{c}$ Carmina Gisbert ${ }^{c}$ \\ and María Belén Picó ${ }^{c^{*}}$
}

BACKGROUND: The grafting of watermelons (Citrullus lanatus) is a common technique that increases yield under stressful soil conditions. The most common rootstocks for watermelons are Cucurbita hybrids. However, they often have a negative impact on fruit quality. Exploiting novel Citrullus germplasm such as citron melon (Citrullus lanatus var. citroides) is an alternative to avoid these quality problems.

RESULTS: Citron melon has been validated as watermelon rootstock, comparing its effects on watermelon quality to those of Cucurbita hybrids. Larger fruits with thicker rinds were observed in fruits from plants grafted onto both citron and Cucurbita rootstocks. The citron melon had no significant effect on flesh sugars or acid profiles compared to non-grafted watermelons, except for an increase in glucose and malic acid content, which also occurred in the Cucurbita rootstocks. The aroma profile of fruits produced on citron melon was similar to that of the non-grafted and self-grafted controls. The citron rootstock did not display the increased levels of (Z)-6-nonen-1-ol (a compound associated with pumpkin-like odors) found in fruits produced with Cucurbita hybrids.

CONCLUSION: The low impact of citron melon rootstock on fruit quality, along with the enhanced resistance against nematodes, make the citron a promising alternative to Cucurbita rootstocks.

Keywords: Citrullus lanatus; fruit quality; grafting; aroma profile. 


\section{INTRODUCTION}

Grafting in watermelon (Citrullus lanatus (Thunb.) Matsum. \& Nakai) production is a common technique that increases yield under stressful conditions (Davis et al., 2008). It is mainly used to control Fusarium wilt, caused by Fusarium oxysporum Schlechtend Fr. F. sp. niveum (EF Sm.; WC Snyder and HN Hans), as well as other damaging soilborne diseases, like Verticillium wilt (Verticillium dahliae Kleb.) (King et al., 2010). It is also an important management strategy that permits a faster response to variable scenarios of abiotic stress than conventional breeding. This technology was first used during the late 1920s in Japan and Korea, and was introduced to different European countries in the late 1980s and later to the USA (Kubota et al., 2008).

The most common commercial rootstocks for watermelons are Cucurbita interspecific hybrids ( $C$. moschata Duchesne $\times C$. maxima Duchesne) and bottle gourd accessions (Lagenaria siceraria Standl.). These rootstocks confer resistance to most of the soilborne fungi affecting watermelon. However, they are susceptible to root-knot nematodes (RKNs, Meloidogyne spp.) (Özarslandan et al., 2011). These pathogens cause extensive damage to watermelon roots and increase the severity of Fusarium wilt in watermelon fields. RKNs used to be controlled in watermelon by fumigation with methyl bromide. However, the removal of methyl bromide from the market has resulted in an increase of the impact of RKNs on watermelon and other cucurbit crops, as the alternative treatments are less effective than this fumigant (Thies et al., 2010).

This situation has caused a spike in the search for resistance in other Cucurbitaceae genera that could lead to the development of alternative rootstocks suitable for managing RKNs in watermelon crops. Some species belonging to the Cucumis genus (C. metuliferus E. Mey. ex Naud. and C. pustulatus Naudin ex Hook. f.) have been reported as being resistant to RKNs (Liu et al., 2015). These rootstocks can be used for watermelons, but are more suitable as rootstocks for other crops belonging to the same genus, such as melon and cucumber (C. melo L. and C. sativus L.) (Sigüenza et al., 2005). Other, more promising materials for grafting watermelons are those belonging to Citrullus lanatus var. citroides (LH Bailey) Mansf. ex Greb., also called citron melon. The citroides group is a group of ancient cultigens from southern Africa that today can be found worldwide, and which is often considered to be an ancestor of cultivated watermelons (Paris, 2015). Citron melons are cultivated around the world mainly for fodder and for the production of fruit preserves. 
Resistance to nematodes, expressed as less galling than that displayed by Cucurbita hybrids and bottle gourd rootstocks, has been reported in some citron melon accessions (Thies et al., 2010), suggesting the usefulness of this group as rootstock for managing RKNs in watermelon (Thies et al., 2015). Some resistant accessions have already been validated in specific conditions (Huitrón et al., 2007), giving higher yields in comparison with C. maxima $\times$ C. moschata commercial rootstocks. Whereas citron melons display lower levels of resistance to Fusarium than hybrid squash and bottle gourd rootstocks, watermelon plants grafted onto citron rootstocks showed enhanced tolerance to Fusarium wilt (Keinath \& Hassell, 2014). Rotation of citron melon with Cucurbita and Lagenaria rootstocks could be an effective practice in the combined management of nematodes and Fusarium (Huitrón et al., 2007; Thies et al., 2015).

Different studies have proven that rootstocks may influence, positively or negatively, scion fruit quality in many vegetables (Condurso et al., 2012; Petropoulos et al., 2012; Rouphael et al., 2010). The most obvious reason for a negative impact is rootstock/scion incompatibility. However, even in compatible combinations different effects are commonly observed. These effects are dependent on the rootstock-scion interaction, which can influence nutrient and water uptake, hormone synthesis, photosynthesis and other metabolic processes (Aloni et al., 2010). It has recently been described that rootstocks induce a differential gene expression in the scion, suggesting that this mechanism may play an important role in mediating the physiological processes of grafted plants (Liu et al., 2016).

Changes in fruit quality as a consequence of grafting can affect the external appearance and/or flesh characteristics, including the chemical composition and organoleptic properties of the fruit. The most common effects on the external appearance of watermelons grafted onto Cucurbita hybrids or L. siceraria rootstocks are increase in fruit weight, rind thickening and increased flesh firmness (Alexopoulos et al., 2007; Davis et al., 2008; Soteriou \& Kyriacou, 2015; Yamasaki et al., 1994). Other clearly negative effects have been reported, such as more fibrous flesh (Muramatsu, 1981), poor color and taste, increased number of yellowish bands and the occurrence of hollow heart (Lee \& Oda, 2003). Changes in mineral composition, sugars, ascorbic acid, citrulline or lycopene content also occur (Alexopoulos et al., 2007; Çandir et al., 2013; Davis \& Perkins-Veazie, 2005; Yamasaki et al., 1994). The impact of grafting on fruit aroma has been studied to a lesser degree, although recent reports show an increase in the concentrations of certain 
aldehyde volatile compounds in watermelon fruits from plants grafted onto interspecific Cucurbita hybrids and Lagenaria rootstocks (Petropoulos et al., 2014). Most of these reported effects depend on the scion-rootstock combination. It is therefore imperative that we evaluate the impact of new putative rootstocks on scion fruit quality.

The aim of this study was to validate the use of a selected citron melon accession collected in Spain as rootstock for watermelon. This accession had been reported previously as highly resistant to nematodes (Gisbert et al., 2017). The effect of this citron melon accession on fruit quality was studied and compared with non-grafted plants, self-grafted plants and plants grafted onto two Cucurbita hybrid rootstocks. The effect of grafting on both external and internal quality, including a detailed analysis of sugars, organic acids and volatile profiles was assessed.

\section{EXPERIMENTAL}

\subsection{Plant material}

The Spanish accession Citrullus lanatus var. citroides BGV0005167 held at the COMAV's Genebank was assayed as a new potential rootstock for watermelon. This accession was collected in Ademuz (Valencia, eastern Spain). It was tested against nematodes under natural conditions, and responded as highly resistant, with a significantly lower number of eggs and nematodes on roots compared to the watermelon scions (Gisbert et al., 2017).

Its behavior as watermelon rootstock (coded as GC) was compared with two C. maxima $\times$ C. moschata hybrids (a new experimental F1 hybrid, coded GMM1, and the commercial F1 Cobalt from Rijk Zwaan, coded GMM2). The experimental hybrid was obtained by crossing the $C$. maxima accession VAV 1860 (Large Warted Hubbard from Australia) $\times C$. moschata PI 550689 (Canada Crookneck squash from the USA). This parental combination was selected because of the high compatibility of the cross as well as the early flowering of the resulting hybrid. Non-grafted watermelons, coded NG, and self-grafted watermelons, coded SG, were included as controls. The watermelon F1 Oneida (RijkZwaan) was used as scion in all treatments. 


\subsection{Field assay}

The approach grafting method was used to graft 24 plants of the watermelon F1 Oneida onto each of the four rootstocks (citron melon, F1 experimental hybrid, F1 Cobalt and F1 Oneida). Once the grafting was consolidated (after 30 days), the plants were transplanted to an experimental field (a field belonging to the company Rijk Zwaan, located in Picassent, Valencia, eastern Spain), along with 24 non-grafted plants of F1 Oneida, used as non-grafted control.

The experiment was carried out in a randomized complete block design with four replications of each rootstock-scion combination and each control (each replication having six plants). Between-row and within-row spacing were $2.0 \mathrm{~m}$ and $1 \mathrm{~m}$, respectively. Plants were furrow irrigated and fertilized according to standard cultural practices. The six plants of each replication were used for fruit sampling. Twenty-four fruits were harvested per combination (six per replication) and were morphologically characterized. Sixteen fruits per combination (four from each replication) were additionally sampled for sugars, acids and aroma analysis. The harvesting of fruits was performed in 2 days and all fruits were characterized over a 5-day period. Fruits were harvested at visual maturity based on external indicators, such as senescent tendrils, ground spot color and external fruit color.

\subsection{Fruit characterization}

Each fruit (24 per treatment) was characterized for the following traits: weight (g), length and width $(\mathrm{cm})$, fruit shape (length/width ratio), rind and flesh thickness ( $\mathrm{mm}$ and $\mathrm{cm}$ ), rind and flesh firmness $\left(\mathrm{kg} \mathrm{cm}^{-2}\right)$ (measured with a digital penetrometer $(8 \mathrm{~mm})$; FHT$803^{\circledR}$, Melrose, MA, USA), total soluble solids (quantified using a digital refractometer, Atago $^{\circledR}$, Tokyo, Japan), pH (measured with pH indicator paper pH 1-14 Merck, Darmstadt, Germany) and flesh color (measured with a colorimeter; Minolta CR-400, Ramsey, NJ, USA using the color parameters Hunter L, a and b).

A $5 \mathrm{~cm}$ cross-section was obtained from the equatorial plane of each of four fruits per replication (16 fruits per treatment). Pericarp and approximately $2 \mathrm{~mm}$ of flesh and seeds 
were discarded. The remaining flesh was homogenized (KRUPS KB720, Groupe Seb Iberica, Barcelona, Spain) and kept frozen at $-80^{\circ} \mathrm{C}$ until metabolite analysis.

\subsection{Reagents for metabolite analysis}

Organic acid standards were prepared from their sodium salts or free acids. The chemicals used were of analytical grade and were purchased from Sigma (St Louis, MO, USA). Glucose, fructose, sucrose and 2,6-pyridine dicarboxylic acid (PDC), hexadimethrine bromide (HDM) and sodium dodecyl sulfate (SDS) were supplied by Sigma. All other chemicals were of analytical grade and were used as received. Individual stock solutions of the analytes $\left(1000 \mu \mathrm{g} \mathrm{mL}^{-1}\right)$ were prepared and stored at $4{ }^{\circ} \mathrm{C}$ until use. Working solutions were prepared daily by diluting the stock solutions with deionized water.

Reference standards of volatile compounds were supplied by Supelco (Sigma-Aldrich and Fluka, Barcelona, Spain) as pure compounds (90-99.5\% purity). Individual standard stock solutions (500 $\left.\mathrm{mg} \mathrm{L}^{-1}\right)$ were prepared in acetone. Then, several mixed standard solutions were obtained by tenfold volume dilution in acetone. Calibration solutions were prepared from working solutions by consecutive volume dilutions with n-hexane to different final concentrations, according to the detector response for each compound (final concentrations ranging from 10 to $5000 \mathrm{ng} \mathrm{mL} \mathrm{L}^{-1}$ ). All standard solutions were stored at $-18^{\circ} \mathrm{C}$ in sealed glass vials (without leaving any headspace) to avoid analyte losses and to ensure reproducibility. Gas chromatography-grade solvents were obtained from Scharlab (Barcelona, Spain). SupelcleanTM ENVI-CarbTM 120-400 mesh, 500 mg SPE tubes, 6 $\mathrm{mL}$ (Supelco) were used as traps.

\subsection{Analysis of sugars and acids}

Fruit samples stored at $-80{ }^{\circ} \mathrm{C}$ were thawed in a refrigerator in complete darkness. They were then centrifuged at $510 \times \mathrm{g}$ for $5 \mathrm{~min}$. The upper phase was diluted (1:20) with deionized water. The solution was filtered using centrifuge tube filters with $0.22 \mu \mathrm{m}$ membranes (Costar® Spin-X®, Corning, Amsterdam) and were subsequently analyzed. 
The sugars sucrose, fructose and glucose, as well as malic, citric and glutamic acids, were quantified by capillary electrophoresis following the method described previously (Baldwin et al., 1998; Cebolla-Cornejo et al., 2012) on an Agilent 7100 system (Agilent Technologies, Waldbronn, Germany) using fused-silica capillaries (Polymicro Technologies, Phoenix, AZ, USA) with $50 \mu \mathrm{m}$ internal diameter, $363 \mu \mathrm{m}$ external diameter, $67 \mathrm{~cm}$ total length and $60 \mathrm{~cm}$ effective length. Capillaries were previously conditioned with the following flushes at $95000 \mathrm{~Pa}$ and $50{ }^{\circ} \mathrm{C}: \mathrm{NaOH} 1 \mathrm{~mol} \mathrm{~L}^{-1}$ (5 min), $\mathrm{NaOH} 1 \mathrm{~mol} \mathrm{~L}^{-1}$ (5 min), and deionized water (Elix 3, Millipore, Billerica, MA, USA) (10 $\min )$. At the beginning of each working session, the capillary was flushed at $20{ }^{\circ} \mathrm{C}$ with the running background electrolyte (BGE) for $30 \mathrm{~min}$. BGE consisted of $20 \mathrm{mmol} \mathrm{L}^{-1} 2,6-$ piridin dicarboxylic acid at $\mathrm{pH} 12.1$ and $0.1 \% \mathrm{w} / \mathrm{v}$ hexadimethrine bromide. Between runs, the capillary was flushed with $58 \mathrm{mmol} \mathrm{L}-1$ SDS (2 min) and running BGE (5 min). Samples were injected hydrodynamically at $3400 \mathrm{~Pa}$ for $10 \mathrm{~s}$. Separations were performed at $-25 \mathrm{kV}$ and $20{ }^{\circ} \mathrm{C}$. Indirect detection was done at $214 \mathrm{~nm}$. Results are expressed in milligrams per kilogram fresh weight $(\mathrm{FW})$. Sucrose equivalents were calculated by multiplying sucrose, glucose and fructose content by $1,0.74$ and 1.73, respectively, and then adding them up (Petropoulos et al., 2014).

\subsection{Analysis of volatiles}

Volatiles were extracted and analyzed following the methodology described previously (Fredes et al., 2016). The extraction was performed in a dynamic headspace system (DHS) using a homemade purge and trap device (Beltran et al., 2006) with commercial (500 mg) SPE cartridges as a trap. Before analysis, the trap cartridges were conditioned with $5 \mathrm{~mL}$ diethyl ether $\left(\mathrm{Et}_{2} \mathrm{O}\right)$ and then with $5 \mathrm{~mL}$-hexane, and were finally vacuum dried for 10 min. A sample amount of $30 \mathrm{~g}$ homogenized fruit was weighed in a $150 \mathrm{~mL}$ flask closed with a glass cap with two connection tubes; the inlet tube was connected to a dry nitrogen gas $\left(\mathrm{N}_{2}\right)$ source and the outlet tube to the trap. Extraction was performed for 49 min with a nitrogen flow rate of $1.6 \mathrm{~L} \mathrm{~min}^{-1}$. Samples were stirred at $7 \times \mathrm{g}$ using a magnetic stir bar and heated to $40^{\circ} \mathrm{C}$.

After extraction, each cartridge was eluted with $5 \mathrm{~mL}$ diethyl ether-hexane (1:1), followed by $5 \mathrm{~mL}$ diethyl ether directly into a graduated glass tube. The extract was evaporated 
under a gentle nitrogen stream at a controlled temperature of $35^{\circ} \mathrm{C}$ to a final volume of 0.5 $\mathrm{mL}$. The final extract was divided into two aliquots in vials with $200 \mu \mathrm{L}$ inserts, sealed and stored in a freezer at $-20{ }^{\circ} \mathrm{C}$ until analysis by gas chromatography-mass spectrometry (GC-MS; ion trap).

A Varian CP-3800 gas chromatograph coupled to an MS detector (Saturn 4000, Varian) was used for analysis of the volatiles included in Table 1 . A $30 \mathrm{~m} \times 0.25 \mathrm{~mm}$ Supelcowax $10(0.25 \mu \mathrm{m}$ film thickness) capillary column was used for the separation, using helium at a constant flow of $1 \mathrm{~mL} \mathrm{~min}^{-1}$ as carrier gas. The temperature program was: $40^{\circ} \mathrm{C}$ for $5 \mathrm{~min}$; increased to $160{ }^{\circ} \mathrm{C}$ at $4{ }^{\circ} \mathrm{C} \mathrm{min}-1$; and finally increased to $250{ }^{\circ} \mathrm{C}$ at $30{ }^{\circ} \mathrm{C} \mathrm{min}{ }^{-1}$, with a final isothermal stage of $1 \mathrm{~min}$ (total chromatographic analysis time $39 \mathrm{~min}$ ). Injection of 1 $\mu \mathrm{L}$ sample in splitless mode (injection port temperature $220^{\circ} \mathrm{C}$ ) was performed using a Varian 8400 autosampler equipped with a $10 \mu \mathrm{L}$ syringe. MS (ion trap) determinations were performed in full-scan mode (m/z scan range 50-200 Da) using electron impact ionization $(70 \mathrm{eV})$ in positive mode and external ionization configuration. The GC-MS interface, ion trap and manifold temperatures were set at 275,190 and $60{ }^{\circ} \mathrm{C}$, respectively. The retention index for all studied compounds was calculated using a standard containing n-alkanes (C7-C30) on a Supelcowax 10 capillary column and following the formula given by Kovats (1958).

Quantitation was carried out by means of external standard calibration curves obtained by using peak areas from the corresponding extracted ion chromatograms for the selected quantitation ion (Q) for each compound (Table 1). Compounds with concentrations exceeding the linearity range were quantified by diluting extracts with n-hexane until reaching the proper concentration. 
Table 1. Volatiles analyzed in the watermelon samples.

\begin{tabular}{|c|c|c|c|c|c|c|c|c|}
\hline$\#$ & Compound name & CAS $^{\mathrm{a}}$ no. & MW & $\begin{array}{c}\text { Rt } \\
(\min )^{b}\end{array}$ & $\begin{array}{c}\text { RI } \\
\text { (SupelcoWax 10) }^{\text {b }}\end{array}$ & Quan Ion $(m / z)^{b}$ & $\begin{array}{l}\text { Linearity } \\
\text { range }\left(\mathrm{ng} \mathrm{mL}^{-1}\right)^{\mathrm{c}}\end{array}$ & $r^{2}$ \\
\hline 1 & Methyl butyrate & $623-42-7$ & 102 & 4,04 & 900 & 43 & $454-2268$ & 0,988 \\
\hline 2 & Methyl-2-methylbutyrate & $868-57-5$ & 116 & 4,55 & 921 & 88 & $23-2270$ & 1,000 \\
\hline 3 & 2-methyl propyl acetate & $110-19-0$ & 116 & 4,67 & 926 & 43 & $111-2765$ & 1,000 \\
\hline 4 & Ethyl butyrate & $105-54-4$ & 116 & 5,34 & 947 & 71 & $265-2648$ & 0,997 \\
\hline 5 & Ethyl-2-methyl butyrate & $7452-79-1$ & 130 & 5,80 & 963 & 57 & $44-2173$ & 0,995 \\
\hline 6 & Butyl acetate & $123-86-4$ & 116 & 6,51 & 985 & 43 & $105-2616$ & 0,999 \\
\hline 7 & Hexanal & $66-25-1$ & 100 & 7,20 & 993 & 56 & $743-7425$ & 0,995 \\
\hline 8 & Propyl butyrate & $105-66-8$ & 130 & 8,24 & 1034 & 43 & $55-2745$ & 1,000 \\
\hline 9 & Ethyl pentanoate & $539-82-2$ & 130 & 8,70 & 1047 & 88 & $25-2508$ & 0,999 \\
\hline 10 & Butyl isobutyrate & $97-87-0$ & 144 & 9,13 & 1059 & 89 & $22-2155$ & 0,999 \\
\hline 11 & Isobutyl butyrate & $539-90-2$ & 144 & 9,58 & 1071 & 71 & $30-2950$ & 1,000 \\
\hline 12 & Ethyl-(E)-2-butanoate & $623-70-1$ & 114 & 9,69 & 1074 & 69 & $26-2596$ & 0,999 \\
\hline 13 & Amyl acetate & $123-86-4$ & 116 & 10,11 & 1086 & 43 & $116-2900$ & 1,000 \\
\hline 14 & Heptanal & $111-71-7$ & 114 & 10,49 & 1096 & 70 & $55-2720$ & 1,000 \\
\hline 15 & Methyl hexanoate & $106-70-7$ & 130 & 10,58 & 1099 & 74 & $26-2643$ & 1,000 \\
\hline 16 & Eucalyptol & $470-82-6$ & 154 & 10,99 & 1111 & 93 & 21- 2083 & 1,000 \\
\hline 17 & Butyl butyrate & $109-21-7$ & 144 & 11,74 & 1131 & 71 & $60-2965$ & 0,988 \\
\hline 18 & Ethyl hexanoate & $123-66-0$ & 144 & 12,32 & 1147 & 88 & $87-2183$ & 1,000 \\
\hline 19 & 1-pentanol & $71-41-0$ & 88 & 12,91 & 1162 & 55 & $128-3195$ & 1,000 \\
\hline 20 & Isoamyl butyrate & $106-27-4$ & 158 & 13,46 & 1178 & 70 & $26-2640$ & 1,000 \\
\hline 21 & Hexyl acetate & $142-92-7$ & 144 & 13,73 & 1188 & 43 & $30-3038$ & 1,000 \\
\hline 22 & Octanal & $124-13-0$ & 128 & 14,27 & 1200 & 69 & $50-2479$ & 0,998 \\
\hline 23 & (Z)-3-hexen-1-ol, acetate & $3681-71-8$ & 142 & 15,30 & 1231 & 67 & $27-2660$ & 1,000 \\
\hline 24 & (E)-2-heptenal & $18829-55-5$ & 112 & 15,48 & 1235 & 83 & $85-2118$ & 1,000 \\
\hline 25 & Ethyl heptanoate & $106-30-9$ & 158 & 15,88 & 1247 & 88 & $30-2980$ & 1,000 \\
\hline 26 & 6-methyl-5-Hepten-2-one & $110-93-0$ & 126 & 15,99 & 1251 & 109 & $21-4260$ & 0,999 \\
\hline 27 & 1-hexanol & $111-27-3$ & 102 & 16,54 & 1267 & 56 & $205-4100$ & 1,000 \\
\hline 28 & Heptyl acetate & $112-06-1$ & 158 & 17,27 & 1288 & 43 & $30-2975$ & 1,000 \\
\hline 29 & (Z)-3-hexen-1-ol & $928-96-1$ & 100 & 17,57 & 1297 & 67 & $51-5050$ & 0,992 \\
\hline 30 & Nonanal & $124-19-6$ & 142 & 17,90 & 1307 & 57 & $108-5420$ & 0,999 \\
\hline 31 & (E)-2-octenal & $2548-87-0$ & 126 & 19,05 & 1343 & 55 & $33-833$ & 0,999 \\
\hline 32 & (Z)-6-nonenal & $2277-19-2$ & 140 & 19,75 & 1366 & 81 & $61-12260$ & 0,988 \\
\hline 33 & Octyl acetate & $112-14-1$ & 172 & 20,60 & 1391 & 43 & $58-2913$ & 1,000 \\
\hline 34 & (E,E)-2,4-heptadienal & 881395 & 110 & 21,17 & 1408 & 81 & $22-2203$ & 1,000 \\
\hline 35 & Decanal & $112-31-2$ & 156 & 21,31 & 1413 & 67 & $25-2469$ & 0,999 \\
\hline 36 & (E,E)-2,4-hexadienoic acid, ethyl ester & 2396-84-1 & 140 & 21,58 & 1423 & 67 & $24-2370$ & 0,999 \\
\hline 37 & Benzaldehyde & $100-52-7$ & 106 & 21,97 & 1436 & 105 & $26-2613$ & 1,000 \\
\hline 38 & (E)-2-nonenal & $18829-56-6$ & 140 & 22,43 & 1450 & 81 & $49-9780$ & 0,982 \\
\hline 39 & Linalool & $78-40-6$ & 154 & 22,89 & 1465 & 93 & $23-2345$ & 0,998 \\
\hline 40 & 1-octanol & $111-87-5$ & 130 & 23,14 & 1474 & 55 & $41-2063$ & 0,999 \\
\hline 41 & Ethyl-3-(methylthio)propanoate & $13327-56-5$ & 148 & 23,37 & 1483 & 74 & $32-3185$ & 1,000 \\
\hline 42 & (E,Z)-2,6-nonadienal & $557-48-2$ & 138 & 23,95 & 1501 & 70 & $95-9460$ & 0,996 \\
\hline 43 & $\beta$-ciclocytral & $5392-40-5$ & 152 & 24,82 & 1533 & 110 & $24-2358$ & 0,996 \\
\hline 44 & Phenylacetaldehyde & $122-78-1$ & 120 & 25,59 & 1559 & 91 & $23-2260$ & 0,941 \\
\hline 45 & 1-nonanol & $143-08-8$ & 144 & 26,15 & 1585 & 55 & $41-4140$ & 0,999 \\
\hline 46 & 2-hydroxybenzaldehyde & $90-02-8$ & 122 & 26,66 & 1594 & 122 & $29-2915$ & 0,997 \\
\hline 47 & (Z)-3-nonen-1-ol & $10340-23-5$ & 142 & 26,80 & 1600 & 67 & $24-4750$ & 1,000 \\
\hline 48 & (E,E)-2,4-nonadienal & $5910-87-2$ & 138 & 27,32 & 1618 & 81 & $22-2155$ & 0,997 \\
\hline 49 & (Z)-6-nonen-1-ol & $35854-86-5$ & 142 & 27,75 & 1634 & 67 & $37-7455$ & 0,999 \\
\hline 50 & Benzyl acetate & $140-11-4$ & 150 & 28,04 & 1646 & 108 & $13-1250$ & 1,000 \\
\hline 51 & 1-decanol & $112-30-1$ & 158 & 29,01 & 1680 & 55 & $83-2075$ & 0,996 \\
\hline 52 & (E,Z)-2,6-nonadien-1-ol & $28069-72-9$ & 140 & 29,15 & 1685 & 67 & $22-2183$ & 0,999 \\
\hline 53 & (E,E)-2,4-decadienal & $25152-84-5$ & 152 & 30,30 & 1727 & 81 & $22-2180$ & 0,996 \\
\hline 54 & Phenethyl acetate & $103-45-7$ & 164 & 30,38 & 1734 & 104 & $25-2452$ & 1,000 \\
\hline 55 & Geranylacetone & $689-67-8$ & 194 & 31,44 & 1773 & 43 & $44-4345$ & 0,997 \\
\hline 56 & Guaiacol & $90-05-1$ & 124 & 31,59 & 1779 & 109 & $26-2638$ & 1,000 \\
\hline 57 & Benzyl Alcohol & $100-51-6$ & 108 & 31,99 & 1795 & 79 & $13-2500$ & 0,999 \\
\hline 58 & 2-phenylethanol & $60-12-8$ & 122 & 32,85 & 1830 & 91 & $13-1250$ & 0,999 \\
\hline 59 & $\beta$-ionone & $14901-07-6$ & 192 & 33,49 & 1856 & 177 & $24-2363$ & 0,999 \\
\hline 60 & Phenol & $108-95-2$ & 94 & 35,24 & 1937 & 94 & $54-2173$ & 0,988 \\
\hline 61 & Eugenol & $97-53-0$ & 164 & 36,99 & 2098 & 164 & $27-2650$ & 0,997 \\
\hline
\end{tabular}

a CAS $=$ Chemical Abstract Service

b Chromatographic parameters (retention time (Rt), retention index (RI) and quantitation ion (Quan Ion)) obtained from GC-MS chromatograms. P\&T extraction using EnviCarb $500 \mathrm{mg}$ cartridges, $30 \mathrm{~g}$ of watermelon sample. Retention index calculated with n-alkanes on Supelcowax 10 (bonded polyethylene glycol) capillary column.

c Linearity range corresponding to the real concentration of standards used for calibration. 


\subsection{Statistical analysis}

Changes in fruit characteristics and sugar and acid content were evaluated using analysis of variance (ANOVA) and least significant difference (LSD) multiple range tests. Changes in the volatile composition of different watermelon genotypes due to grafting were evaluated jointly using multivariate analysis of variance (MANOVA) and MANOVA biplot. In the MANOVA biplot subspace, the similarity between grafting treatments can be measured as an inverse function of their distance on the graph. The angle between variables can be interpreted as an approximation of their correlation. The inner product of a group marker with a variable marker approximates the mean of the kth group on the jth variable allowing for the characterization of the differences between groups. Univariate Bonferroni confidence circles are added to the group markers in such a way that the projections of the circles onto the direction representing a given variable represent an approximate confidence interval. The significance of the difference between groups with regard to a particular variable can be established by checking the overlap of their projections. The procedure is conservative in the sense that if no overlap is found it can be concluded that there is a significant difference, but if there is an overlap a significant difference may be found along another direction in the multidimensional space. All MANOVA biplot calculations and graphs were made with MultBiplot free-licensed software (VicenteVillardon, 2014).

\section{RESULTS}

\subsection{Effect of grafting on fruit morphology and flesh properties}

Comparison of fruits from non-grafted and self-grafted watermelon plants indicates that the grafting process itself does not alter fruit size and shape or essential flesh properties such as firmness, soluble solids content and $\mathrm{pH}$ (Table 2). The only parameter affected by self-grafting was flesh color, with fruits from grafted plants having values for $a$ and $b$ color parameters that were significantly lower than those of non-grafted plants, which means less intensity of the red and yellow flesh color components. 
Unlike self-grafting, grafting onto non-watermelon rootstocks altered fruit size (Table 2). In fact, both types of rootstock - citron melon (GC) and the two F1 Cucurbita hybrids (GMM1 and GMM2) - increased fruit weight significantly (in comparison to both selfgrafted and to non-grafted watermelons). This increment was $24 \%$ on average, and was associated with the production of wider and longer fruits, without significant fruit shape alterations. In addition, both citron melon and Cucurbita rootstocks yielded fruits with thicker rinds than SG and NG, which was also accompanied by an increase in flesh thickness (ranging from 16.9 to $17.2 \mathrm{~cm}$ in grafted versus $16.0 \mathrm{~cm}$ in non-grafted plants). These rootstocks resulted in fruits with firmer flesh than those produced by self-grafted plants.

Table 2. Characteristics of watermelon fruits (Citrullus lanatus F1 Oneida) harvested from non-grafted plants (NG), self-grafted plants (SG) and plants grafted the experimental Cucurbita (C. maxima $\times$ C. moschata) hybrid F1 (GMM1), the commercial Cucurbita hybrid Cobalt (GMM2) and one new experimental citron melon (Citrullus lanatus var. citroides) (GC).

\begin{tabular}{|c|c|c|c|c|c|}
\hline Fruit weight (g) & $3157.8 \mathrm{a}$ & $3321.7 \mathrm{a}$ & $3958.7 b$ & $4160.2 b$ & $3970.9 b$ \\
\hline Fruit width FW (cm) & $17.6 \mathrm{a}$ & $17.9 \mathrm{a}$ & $18.8 b$ & $19.2 b$ & $18.8 b$ \\
\hline Fruit shape (FL/FW) & $1.16 \mathrm{ab}$ & $1.17 \mathrm{~b}$ & $1.12 \mathrm{a}$ & $1.17 \mathrm{~b}$ & $1.19 \mathrm{~b}$ \\
\hline Flesh thickness (cm) & $16.0 \mathrm{a}$ & $16.3 \mathrm{ab}$ & $16.9 \mathrm{bc}$ & $17.2 \mathrm{c}$ & $17.0 \mathrm{bc}$ \\
\hline Rind firmness $\left(\mathrm{kg} \mathrm{cm}^{-2}\right)$ & $12.37 \mathrm{a}$ & $12.89 \mathrm{a}$ & $12.98 \mathrm{a}$ & $12.65 \mathrm{a}$ & $12.92 \mathrm{a}$ \\
\hline Flesh firmness $\left(\mathrm{kg} \mathrm{cm}^{-2}\right)$ & $1.21 \mathrm{ab}$ & $1.04 \mathrm{a}$ & $1.38 \mathrm{~b}$ & $1.27 \mathrm{~b}$ & $1.38 \mathrm{~b}$ \\
\hline Total soluble solids ( $\left.{ }^{\circ} \mathrm{Brix}\right)$ & $9.6 \mathrm{ab}$ & $9.3 \mathrm{a}$ & $9.6 \mathrm{ab}$ & $10.1 \mathrm{bc}$ & $10.2 \mathrm{c}$ \\
\hline pH & $5.2 \mathrm{ab}$ & $5.3 b$ & $5.1 \mathrm{ab}$ & $5.2 \mathrm{ab}$ & $5.1 \mathrm{a}$ \\
\hline
\end{tabular}

Different letters in the same row indicate significant differences $(L S D, P=0.05)$.

The citron melon was the only rootstock that significantly increased the total soluble solids content compared to SG and NG watermelons (10.2 ${ }^{\circ}$ Brix versus 9.3 and $\left.9.6^{\circ} \mathrm{Brix}\right)$. Of all the fruits harvested from grafted plants, fruits from GC plants had the most similar flesh color to the non-grafted watermelons. In fact, unlike the fruits from self-grafted plants and from plants grafted onto the Cucurbita hybrids, this rootstock did not cause a reduction in the values of the Hunter color parameters. 


\subsection{Effect of grafting on flesh sugar and acid content}

As occurred with fruit morphology and flesh properties, we did not find significant differences in acid content between fruits of non-grafted and self-grafted plants (Table 3). However, some significant changes in these metabolites were found in fruits from plants grafted onto non-watermelon rootstocks. Both citron melon and Cucurbita hybrids significantly increased the malic acid content compared with non-grafted plants (Table 3). Citron melon did not alter the citric and glutamic acid content in comparison to fruits from non-grafted and self-grafted plants, whereas fruits from plants grafted onto Cucurbita hybrids had lower content of these acids than those of citron melon-grafted plants.

Fructose content was not affected by grafting (Table 2). However, the citron rootstock was the non-watermelon rootstock that produced fruits with the highest glucose amounts significantly higher than fruits from NG plants - which is consistent with the high value of total soluble solids content that was observed in fruits from GC plants. Sucrose measurements were more variable. No significant differences were observed between nongrafted plants and those using either citron melon or the commercial Cucurbita rootstock. Only the experimental Cucurbita hybrid rootstock significantly reduced sucrose content. Similar results were observed with sucrose equivalents.

Table 3. Sugars and acids concentrations of watermelon fruits (Citrullus lanatus cv. F1 Oneida) harvested from non-grafted plants (NG), self-grafted plants (SG) and plants grafted onto the experimental Cucurbita (C. maxima $\times$ C. moschata) hybrid F1 (GMM1), the commercial Cucurbita hybrid Cobalt (GMM2) and one new experimental citron melon (Citrullus lanatus var. citroides) (GC).

\begin{tabular}{|c|c|c|c|c|c|}
\hline Malic acid (mg kg-1) & $163.0 \mathrm{a}$ & $195.1 \mathrm{ab}$ & $219.9 b$ & $220.1 b$ & $230.5 b$ \\
\hline Glutamic acid (mg kg-1) & $2.2 \mathrm{abc}$ & $3.6 b c$ & $0.5 \mathrm{a}$ & $0.9 \mathrm{ab}$ & $4.1 \mathrm{c}$ \\
\hline Fructose $\left(\mathbf{m g ~ k g}^{-1}\right)$ & $2411.6 \mathrm{a}$ & $2696.6 \mathrm{a}$ & $2305.6 \mathrm{a}$ & $2558.0 \mathrm{a}$ & $2814.8 \mathrm{a}$ \\
\hline Sucrose $\left(\mathrm{mg} \mathrm{kg}^{-1}\right)$ & $1185.4 \mathrm{~b}$ & $1651.3 \mathrm{c}$ & $688.3 \mathrm{a}$ & $1118.1 \mathrm{ab}$ & $1155.5 b$ \\
\hline Sucrose equivalents $\left(\mathrm{mg} \mathrm{kg}^{-1}\right)$ & $6564.3 \mathrm{ab}$ & $7737.2 b$ & $5989.7 \mathrm{a}$ & $6945.4 \mathrm{ab}$ & $7566.8 \mathrm{~b}$ \\
\hline
\end{tabular}

Different letters in the same row indicate significant differences $(L S D, P=0.05)$. 


\subsection{Effect of grafting on flesh aroma profile}

Of the 61 volatiles analyzed, 32 were found in quantifiable amounts (Table 4). The following 29 compounds were analyzed, but their presence was not detected in the samples: (Z)-3-hexen-1-ol acetate, ethyl hexanoate, 1-decanol, 2-methyl propyl acetate, 2hydroxy-benzaldehyde, amyl acetate, benzyl acetate, butyl acetate, butyl isobutyrate, diethyl carbonate, ethyl heptanoate, ethyl pentanoate, ethyl-(E)-2-butanoate, ethyl-3(methylthio)propanoate, eucalyptol, eugenol, guaiacol, heptyl acetate, hexyl acetate, isoamyl butyrate, isobutyl butyrate, linalool, methyl butyrate, methyl hexanoate, methyl-2methyl butyrate, phenol, butyl butyrate, phenylethyl acetate and propyl butyrate.

Table 4. Mean contents of the volatiles quantified in watermelon fruits (Citrullus lanatus cv. F1 Oneida) harvested from non-grafted plants (NG), self-grafted plants (SG) and plants grafted onto the experimental Cucurbita (C. maxima $\times$ C. moschata) hybrid F1 (GMM1), the commercial Cucurbita hybrid Cobalt (GMM2) and one new experimental citron melon (Citrullus lanatus var. citroides) (GC).

\begin{tabular}{|c|c|c|c|c|c|c|}
\hline$\#$ & Compound name & Non-grafted (NG) & Self-grafted (SG) & $\begin{array}{l}\text { Cucurbita F1 } \\
\text { experimental (GMM1) }\end{array}$ & $\begin{array}{l}\text { Cucurbita F1 } \\
\text { Cobalt (GMM2) }\end{array}$ & $\begin{array}{l}\text { Citron melon } \\
\text { (GC) }\end{array}$ \\
\hline 1 & (E)-2-Heptenal & 4.7 & 4.4 & 3.7 & 6.0 & 5.8 \\
\hline 2 & (E.Z)-2.6-Nonadienal & 284.2 & 226.5 & 382.6 & 243.2 & 367.2 \\
\hline 5 & (Z)-6-Nonenal & 94.6 & 89.1 & 160.1 & 97.0 & 173.2 \\
\hline 7 & (E)-2-Nonenal & 501.6 & 413.4 & 347.0 & 504.2 & 559.1 \\
\hline 8 & (E)-2-Octenal & 7.2 & 5.8 & 3.5 & 6.1 & 6.4 \\
\hline 11 & (E.E)-2.4-Nonadienal & 2.6 & 2.6 & 1.3 & 3.0 & 2.8 \\
\hline 12 & (E.Z)-2.6-Nonadien-1-ol & 24.4 & 16.2 & 37.0 & 14.9 & 18.4 \\
\hline 13 & (Z)-3-Nonen-1-ol & 1251.9 & 1366.7 & 1096.4 & 1928.0 & 1122.7 \\
\hline 14 & (Z)-6-Nonen-1-ol & 53.1 & 49.5 & 130.9 & 131.9 & 70.4 \\
\hline 16 & 1-Hexanol & 60.0 & 76.7 & 74.3 & 88.7 & 48.9 \\
\hline 23 & 6-methyl-5-Hepten-2-one & 53.1 & 50.8 & 29.8 & 45.3 & 42.6 \\
\hline 25 & Benzaldehyde & 3.4 & 3.3 & 1.0 & 2.1 & 2.7 \\
\hline 26 & Benzyl Alcohol & 16.6 & 15.8 & 8.8 & 11.3 & 12.1 \\
\hline 28 & $\beta$-Ionone & 4.0 & 3.6 & 1.8 & 2.8 & 2.8 \\
\hline 29 & $\beta$-cyclocitral & 1.2 & 1.1 & 0.5 & 0.9 & 0.8 \\
\hline 32 & Decanal & 5.2 & 4.5 & 4.1 & 4.3 & 4.3 \\
\hline 34 & Ethyl butanoate & 50.3 & 42.3 & 36.1 & 54.3 & 34.3 \\
\hline 38 & Ethyl-2-methyl butyrate & 7.1 & 7.6 & 6.8 & 6.8 & 5.8 \\
\hline 42 & Geranylacetone & 134.9 & 143.2 & 85.0 & 120.4 & 125.7 \\
\hline 44 & Heptanal & 3.4 & 3.3 & 2.6 & 3.5 & 3.5 \\
\hline 46 & Hexanal & 104.1 & 109.8 & 82.4 & 90.4 & 120.5 \\
\hline 54 & Nonanal & 207.8 & 268.0 & 194.1 & 189.6 & 284.0 \\
\hline
\end{tabular}

Values are expressed as $\mathrm{ng}^{-1}$ fresh weight. 
Considering the complexity of the analysis, a MANOVA biplot (Figure 1) was selected in order to elucidate the precise differences in the profile of aroma volatiles. The individual samples of each combination were highly clustered in the biplot and separated from those of the other combinations. MANOVA confirmed the significance of the effect that the rootstock had on the aroma volatile profile of watermelon (Roy's greatest root test, $P<$ $10^{-3}$ ). The first axis explained $43 \%$ of the variance and the second $31 \%$. The Bonferroni circles whose projections on the vector of a certain compound do not overlap represent statistically significant differences. Few differences in the volatile profile were found between the non-grafted and the self-grafted controls, which was consistent with the limited effect of self-grafting as described earlier (Figure 1).

Fruits from NG and SG plants stand out for their elevated accumulation of several compounds, including those deriving from the carotenoid degradation pathway, such as geranylacetone, 6-methyl-5-hepten-2-one, $\beta$-ionone and $\beta$-cyclocitral (Table 4). Significant effects on flesh volatile profiles were observed in fruits from plants grafted onto the Cucurbita hybrids (GMM1 and GMM2) and GC (Figure 1) compared to the controls. These grafting combinations showed circles that do not overlap among themselves nor with $\mathrm{NG}$ or SG, indicating different aroma profiles depending on the rootstock. The greatest differences to NG and SG were found in fruits from plants grafted onto the Cucurbita hybrids. In general, low accumulation of volatile compounds, with high amounts of (Z)-6-nonenal, (E-Z)-2-6-nonadien-1-ol and (E-Z)-2-6-nonadienal, were observed in fruits from GMM1-grafted plants (Figure 1 and Table 4), whereas the GMM2 rootstock resulted in higher accumulation of several alcohols, including 1-nonanol, 1octanol, 1-hexanol and (Z)-3-nonen-1-ol. Both Cucurbita hybrids stood out for their accumulation of (Z)-6-nonen-1-ol. In fact, the vector for this compound represented the most important differences from the controls. Watermelon fruits from GC plants showed a more similar volatile profile to the SG and NG controls, with an intermediate position in the MANOVA biplot between the controls and GMM1 (Figure 1). The differences between controls and the CG rootstock were related to the higher accumulation of (Z)-6nonenal, $(E)$-2-nonenal, $(Z)$-6-nonen-1-ol, (E-Z)-2-6-nonadienal and (E,E)-2-4-heptadienal, as well as the lower accumulation of carotenoid-derived volatiles in fruits from plants grafted onto the citron melon rootstock (Figure 1 and Table 4). 


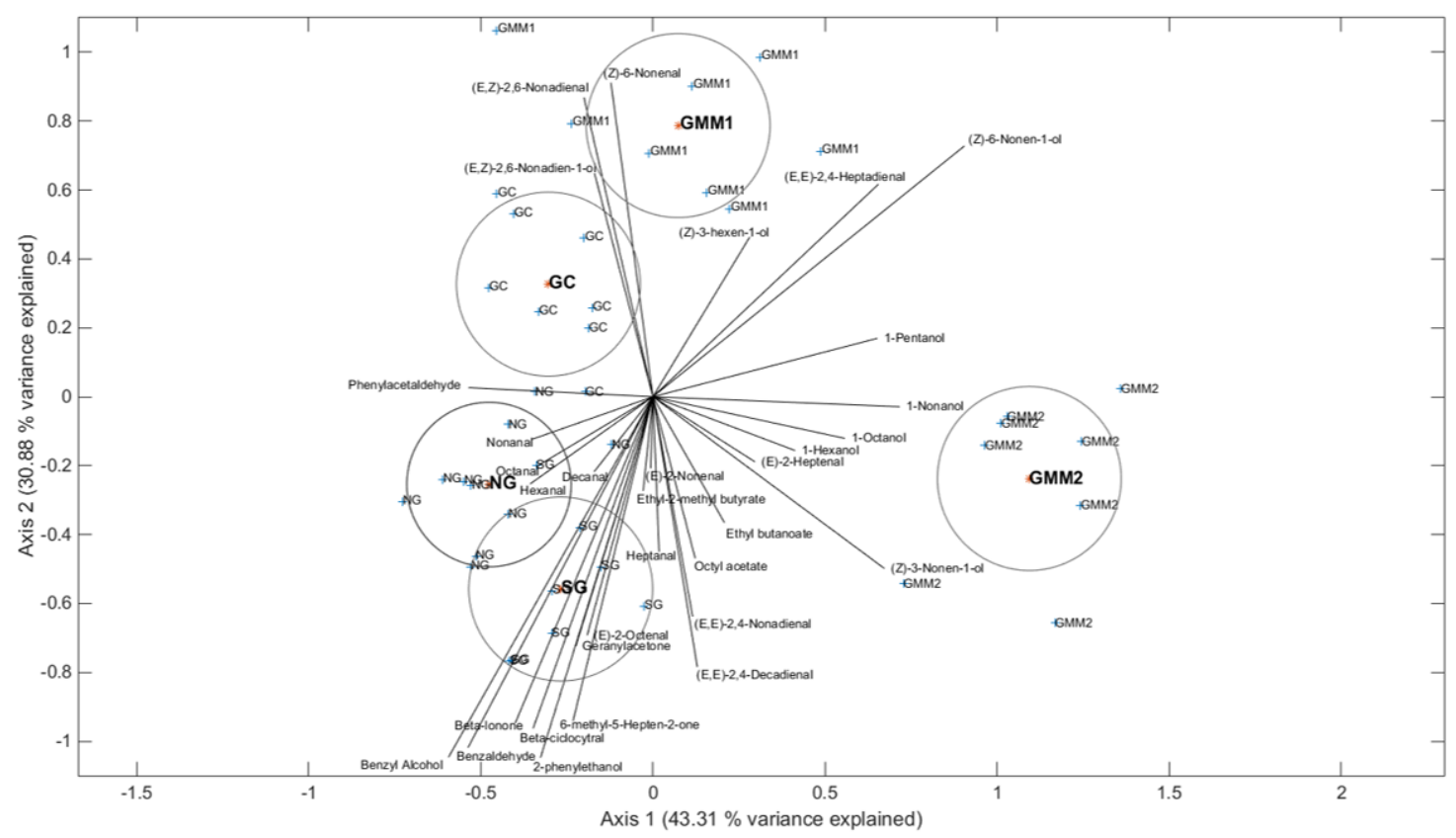

Figure 1. MANOVA biplot of fruit volatiles. Circles represent Bonferroni confidence intervals for the mean value of each material. In all cases the scion corresponds to $\mathrm{cv}$. 'Oneida RZ'. NG: 'Oneida RZ' non-grafted; SG: self-grafted; GC: grafted on citron melon; GMM1: grafted on experimental Cucurbita F1 hybrid; GMM2: grafted on 'Cobalt RZ' a commercial Cucurbita F1 hybrid.

\section{DISCUSSION}

Fruit quality is a complex trait that involves external and internal parameters that are determined to satisfy the consumers' preferences. In watermelon, the main quality-related properties are fruit morphology and flesh texture, color, flavor and aroma. Since fruit quality can be negatively modified by grafting (Rouphael et al., 2010), it is important to evaluate for these traits in each new rootstock-scion combination, such as that of citron melon-watermelon. The use of citron as rootstock is very promising because of its high level of resistance to nematodes (Huitrón et al., 2007; Thies et al., 2015).

In this paper, we have shown that citron melons cause a range of effects in watermelon fruits that are also observed in plants grafted onto Cucurbita hybrids, but have less impact than these commonly used rootstocks on certain quality traits. 
The common effects of citron and Cucurbita rootstocks are mainly related to the production of bigger watermelon fruits. Better yields associated with both larger fruits as well as the increase of fruit number per plant have often been reported for watermelons grafted onto Cucurbita hybrids, in both Fusarium-infested and non-infested soils (Petropoulos et al., 2014). Only a few reports have studied these effects in watermelon plants grafted onto citron rootstocks, and have indicated an increase in yield in citron melon-grafted watermelons cultivated in soils infested with nematodes, but they do not clearly report an increase in fruit size (Huitrón et al., 2007; Thies et al., 2015).

In agreement with what is commonly found in watermelon grafting reports (Colla et al., 2006; Rouphael et al., 2010; Soteriou \& Kyriacou, 2015), the changes that we observed in fruit size did not cause important fruit shape alterations, although they were associated with the thickening of the watermelon rind (Alexopoulos et al., 2007; Soteriou \& Kyriacou, 2015). In our assay, the nearly $2 \mathrm{~mm}$ increase in rind thickness was associated with a higher flesh content (about $1 \mathrm{~cm}$ wider flesh), which minimized the negative impact of this alteration.

Another change often reported as a consequence of grafting watermelons onto different common Cucurbita interspecific hybrids is the increase in flesh firmness (Davis et al., 2008; Yamasaki et al., 1994). This is an important trait as it is related to fruit quality and might also influence postharvest behavior. Flesh firmness is an indicator of ripeness in watermelon. In the current study, all experimental plots were harvested in 2 days, when the fruits of all combinations showed external indicators of maturity(Vinson et al., 2010). The fact that we did not find significant differences in fruit firmness between either the Cucurbita or the citron-grafted watermelons and the fruits from non-grafted plants may suggest that we harvested fruits at a similar maturity state, which is essential in order to assess and compare the quality of fruits from the different treatments.

The development of flesh color might also be associated with the ripening stage. Variable effects of rootstocks on watermelon flesh color have been reported (Davis \& PerkinsVeazie, 2005; Gama et al., 2013; Soteriou et al., 2014). Our results showed a certain reduction in the redness and yellowness of flesh color, which affected both the self-grafted and the Cucurbita-grafted plants, whereas the citron melon rootstocks resulted in a more similar color profile to that of the non-grafted control.

Citron melons seem to affect fruit acidity and sweetness less negatively than Cucurbita hybrids. The balance between flesh sweetness and acidity represents a central parameter in 
determining fruit flavor. Even though all non-watermelon rootstocks increased malic acid concentrations, the citric and glutamic acid content of the fruits harvested in citron melongrafted plants were similar to those of the fruits from non-grafted plants, whereas Cucurbita hybrids reduced the content of the two acids. There are limited studies on the modification of watermelons' acidic profile as a consequence of grafting, and most indicate higher titratable acidity in fruits from grafted plants (Gama et al., 2013; Soteriou et al., 2014).

Regarding the effect on sugar content, our results are in agreement with those found by Colla et al. and Soteriou et al. who found similar sugar compositions in fruits from grafted and non-grafted plants. The relative sweetness of fructose is greater than that of sucrose (Baldwin et al., 1998), which makes the fact that grafting had no effect on fructose content quite favorable, as there were higher concentrations of fructose than glucose and sucrose in all combinations. However, we found a non-desirable effect of grafting, as it reduced the sucrose content. This effect was variable in the Cucurbita hybrids, as it only affected the fruits collected from plants with the experimental Cucurbita rootstock, whereas the commercial Cobalt did not decrease sucrose levels compared to non-grafted plants. Despite this sucrose reduction, none of these Cucurbita rootstocks differed from non-grafted plants in sucrose equivalents. Sucrose equivalents are calculated in order to correct the concentration of each sugar with its sweetening power. This variable has been found, in other crops (Baldwin et al., 1998), to be more correlated with sweetness perception than sugar concentration. Therefore, and as was previously stated for other watermelonCucurbita hybrid combinations, it remains unclear whether reductions in specific sugars will really be perceived by consumers if no differences are detected in sucrose equivalents (Kyriacou et al., 2016). Previous studies also show variable results, reporting both reductions and no effects in sugar content as a consequence of grafting in Cucurbita hybrids and Lagenaria rootstocks, depending on the environment, ploidy level of the scion and stage of fruit development at harvesting (Alexopoulos et al., 2007; Davis et al., 2008; Rouphael et al., 2010). Our study shows that the fruits harvested from plants grafted onto citron melon rootstocks retain the highest levels of all sugars, in addition to the highest level of sucrose equivalents, similar to those found in the non-grafted plants.

Even though grafting in watermelon has been practiced since 1920, there is almost no information available on the effect of rootstock on the aroma profile. Recently, Petropoulos et al. reported the effect of grafting onto TZ 148 (C. moschata $\times$ C. maxima hybrid) and 
Dias F1 (Lagenaria hybrid) on the aroma profile of two varieties of watermelon used as scion (Obla F1: seeded watermelon; and Vanessa F1: unseeded mini watermelon). The use of the Cucurbita rootstock increased the levels of the seven volatiles they quantified in the seeded watermelon, including (Z)-6-nonenal, nonanal, (E,Z)-2,6-nonadienal, (Z,Z)-3,6nonadien-1-ol and (E)-2-nonenal.

In our work, a much higher number of volatiles were quantified, including four of those studied by Petropoulos et al. ((Z)-6-nonenal, (E)-2-nonenal, nonanal, (E,Z)-2,6nonadienal). Two of these compounds ((Z)-6-nonenal and (E,Z)-2,6-nonadienal) have been found to be among the most abundant volatiles in seedless watermelons, and have been associated with melon-like and cucumber-like aromas, respectively (Beaulieu \& Lea, 2006). The higher values of these compounds found in fruits from plants grafted onto Cucurbita hybrids were interpreted by Petropoulos et al. as a negative effect of these rootstocks on the volatile profile of watermelons.

Our results also clearly suggest that the rootstock used has an important effect on the volatile profile of the watermelons produced. We also found increased amounts of the two compounds that confer melon- and cucumber-like aromas in the experimental Cucurbita hybrid, but not in the commercial rootstock, which had similar values to that of self- and non-grafted plants.

The volatile profiles of fruits harvested from plants grafted onto Cucurbita hybrids most notably show the highest levels of (Z)-6-nonen-1-ol. This compound confers pumpkin-like odors (Leffingwell \& Associates, 2016), which is detrimental to fruit quality. In fact, in sliced watermelon, the concentration of (Z)-6-nonen-1-ol increases, and it has been suggested that it may be partially related to the pumpkin-like off-odors and squash-like offflavors often attributed to overripe whole watermelon (Saftner et al., 2007). This effect could be a consequence of a higher accumulation of these compounds, which are specific to the Cucurbita rootstocks, or possibly to a faster ripening process in these grafted plants. Considering that these fruits showed similar values of several ripening-related parameters (including rind and flesh firmness, $\mathrm{pH}$ and sugar content) to the control fruits, the first explanation may be the most probable. Also, as King et al. suggested, the 'squash' flavor may arise when the fruits are harvested too soon, but in our case the occurrence of external signals of fruit ripening and $\mathrm{pH}$ and sugar values do not support this explanation.

Fruits harvested from plants grafted onto citron melons also have high amounts of the volatile compounds associated with melon- and cucumber-like aromas ((Z)-6-nonenal and 
(E,Z)-2,6-nonadienal). However, they have much lower amounts of the squash-like aromaassociated compound (Z)-6-nonen-1-ol than fruits from Cucurbita hybrids.

One of the main differential characteristics of the aromas of non-grafted and self-grafted watermelons is that they have high levels of geranylacetone, 6-methy-5-hepten-2-one and $\beta$-cyclocitral in comparison with the other rootstocks. These compounds originate from the degradation of carotenoids in tomato and watermelon (Grassi et al., 2013; E Lewinsohn et al., 2005). Specifically, 6-methy-5-heten-2-one and geranylacetone seemingly derive from lycopene and other noncyclic tetraterpenoids, whereas $\beta$-ionone and $\beta$-cyclocitral derive from $\beta$-carotene (E. Lewinsohn et al., 2005).

This result correlated with the observed differences in fruit color, as the presence of these volatiles has been related to total carotenoid and lycopene content in watermelon (Sharma et al., 2008). In fact, the experimental Cucurbita hybrid with the strongest impact on flesh yellowness (in b parameter) had lower levels of these carotenoid-derived volatiles. However, this does not explain the differences observed in flesh redness (a parameter) between fruits from non-grafted and self-grafted plants. Fruits from GC plants have only slightly lower amounts of these compounds than non-grafted and self-grafted watermelons, and are similar to those of the plants grafted onto the commercial Cucurbita hybrid.

Overall, grafting watermelon cultivars onto resistant rootstocks is a highly recommendable practice as it enhances the field performance of the scions, but the negative impact on fruit quality can prevent the use of some rootstock-scion combinations. Our results confirm the occurrence of various effects on fruit quality, including a relevant impact on aroma profile, which is dependent on the rootstocks, thereby confirming the existence of variation within the Cucurbita hybrids and the differential effect of citron melons. The use of the citron melon rootstock from the same Citrullus genus produces a more similar volatile profile to that of non-grafted plants. To our knowledge, this paper is the first to describe the effect of citron melon rootstock on watermelon quality properties such as acid, sugar and aroma profiles. The similar volatile profile produced as compared to the controls seems to indicate that citron melon would be a good alternative to the classic Cucurbita hybrids. 


\section{CONCLUSIONS}

The use of citron melon rootstock may represent an interesting alternative to the classic Curcurbita hybrids. The use of this rootstock compared to non-grafted watermelon has a limited effect on the sugar and acid profiles of watermelons or may even improve them slightly. On the other hand, the aroma volatile profile, when using citron melon rootstock, is more similar to the non-grafted control than the commonly used Cucurbita hybrids. Additionally, the use of Cucurbita hybrids increases (Z)-6-nonen-1-ol, which may be detrimental to watermelon flavor, an effect not observed in citron melon. The variability detected in the aroma profiles of different Cucurbita hybrids also suggests that the effect of rootstock on the aroma profile should be considered in rootstock breeding programs.

\section{ACKNOWLEDGEMENTS}

This study was partially supported by the project funded by the Ministerio de Economía y Competitividad AGL2014-53398-C2-2-R (jointly funded by FEDER).

\section{REFERENCES}

Alexopoulos, A.A., Kondylis, A., Passam, H.C., 2007. Fruit yield and quality of watermelon in relation to grafting. J. Food, Agric. Environ. 5, 178-179.

Aloni, B., Cohen, R., Karni, L., Aktas, H., Edelstein, M., 2010. Hormonal signaling in rootstock-scion interactions. Sci. Hortic. 127, 119-126.

Baldwin, E.A., Scott, J.W., Einstein, M., Malundo, T.M.M., Carr, B.T., Shewfelt, R.L., Tandon, K.S., 1998. Relationship between sensory and instrumental analysis for tomato flavor. J.Amer.Soc.Hort.Sci. 123, 906-915.

Beaulieu, J.C., Lea, J.M., 2006. Characterization and semiquantitative analysis of volatiles in seedless watermelon varieties using solid-phase microextraction. J. Agric. Food Chem. 54, 7789-7793. 
Beltrán, J., Serrano, E., López, F.J., Peruga, A., Valcárcel, M., Roselló, S., 2006. Comparison of two quantitative GC-MS methods for analysis of tomato aroma based on purge-and-trap and on solid-phase microextraction. Anal. Bioanal. Chem. 385, $1255-1264$.

Çandir, E., Yetişir, H., Karaca, F., Üstün, D., 2013. Phytochemical characteristics of grafted watermelon on different bottle gourds (Lagenaria siceraria) collected from the Mediterranean region of Turkey. Turkish J. Agric. For. 37, 443-456.

Cebolla-Cornejo, J., Valcárcel, M., Herrero-Martínez, J.M., Roselló, S., Nuez, F., 2012. High efficiency joint CZE determination of sugars and acids in vegetables and fruits. Electrophoresis 33, 2416-2423.

Colla, G., Rouphael, Y., Cardarelli, M., Rea, E., 2006. Effect of salinity on yield, fruit quality, leaf gas exchange, and mineral composition of grafted watermelon plants. HortScience 41, 622-627.

Condurso, C., Verzera, A., Dima, G., Tripodi, G., Crinò, P., Paratore, A., Romano, D., 2012. Effects of different rootstocks on aroma volatile compounds and carotenoid content of melon fruits. Sci. Hortic. 148, 9-16.

Davis, A.R., Perkins-Veazie, P., 2005. Rootstock effects on plant vigor and watermelon fruit quality. Cucurbit Genet. Coop. Rep. 28-29, 39-42.

Davis, A.R., Perkins-Veazie, P., Sakata, Y., López-Galarza, S., Maroto, J.V., Lee, S.G., Huh, Y.C., Sun, Z., Miguel, A., King, S.R., Cohen, R., Lee, J.M., 2008. Cucurbit grafting. CRC. Crit. Rev. Plant Sci. 27, 50-74.

Fredes, A., Sales, C., Barreda, M., Valcárcel, M., Roselló, S., Beltrán, J., 2016. Quantification of prominent volatile compounds responsible for muskmelon and watermelon aroma by purge-and-trap extraction followed by gas chromatographymass spectrometry determination. Food Chem. 190, 689-700.

Gama, R.N.C. de S., Dias, R.C.S., Alves, J.C. da S.F., Damaceno, L.S., Teixeira, F.A., Barbosa, G. da S., 2013. Taxa de sobrevivência e desempenho agronômico de melancia sob enxertia. Hortic. Basileira 31, 128-132.

Gisbert, C., Gammoudi, N., Munera, M., Giné, A., Pocurull, M., Sorribas, F.J., Picó, M.B., 2017. Evaluation of two potential Cucumis spp. resources for grafting melons. Acta Hortic. 1151, 157-162. 
Grassi, S., Piro, G., Lee, J., Zheng, Y., Fei, Z., Dalessandro, G., Giovannoni, J.J., Lenucci, M.S., 2013. Comparative genomics reveals candidate carotenoid pathway regulators of ripening watermelon fruit. BMC Genomics 14, 781.

Huitrón, M.V., Diaz, M., Diánez, F., Camacho, F., 2007. The effect of various rootstocks on triploid watermelon yield and quality. J. Food, Agric. Environ. 5, 344-348.

Keinath, A.P., Hassell, R.L., 2014. Suppression of Fusarium wilt caused by Fusarium oxysporum f. sp. niveum race 2 on grafted triploid watermelon. Plant Dis. 98, 13261332.

King, S.R., Davis, A.R., Zhang, X., Crosby, K., 2010. Genetics, breeding and selection of rootstocks for solanaceae and cucurbitaceae. Sci. Hortic. 127, 106-111.

Kovats, E., 1958. Gas-chromatographische charakterisierung organischer verbindungen. Teil 1: retentionsindices aliphatischer halogenide, alkohole, aldehyde und ketone. Helv. Chim. Acta 41, 1915-1932.

Kubota, C., McClure, M.A., Kokalis-Burelle, N., Bausher, M.G., Rosskopf, E.N., 2008. Vegetable grafting: history, use, and current technology status in North America. Hortscience 43, 1664-1669.

Kyriacou, M.C., Soteriou, G.A., Rouphael, Y., Siomos, A.S., Gerasopoulos, D., 2016. Configuration of watermelon fruit quality in response to rootstock-mediated harvest maturity and postharvest storage. J. Sci. Food Agric. 96, 2400-2409.

Lee, J.M., Oda, M., 2003. Grafting of herbaceous vegetable and ornamental crops. Hortic. Rev. (Am. Soc. Hortic. Sci). 28, 61-124.

Leffingwell \& Associates, 2016. Alkenols and molecular structures. [WWW Document]. URL http://www.leffingwell.com/alkenol.htm. (accessed 1.10.2016).

Lewinsohn, E., Sitrit, Y., Bar, E., Azulay, Y., Ibdah, M., Meir, A., Yosef, E., Zamir, D., Tadmor, Y., 2005. Not just colors: carotenoid degradation as a link between pigmentation and aroma in tomato and watermelon fruit. Trends Food Sci. Technol. $16,407-415$.

Lewinsohn, E., Sitrit, Y., Bar, E., Azulay, Y., Meir, A., Zamir, D., Tadmor, Y., 2005. Carotenoid pigmentation affects the volatile composition of tomato and watermelon fruits, as revealed by comparative genetic analyses. J. Agric. Food Chem. 53, 31423148. 
Liu, B., Ren, J., Zhang, Y., An, J., Chen, M., Chen, H., Xu, C., Ren, H., 2015. A new grafted rootstock against root-knot nematode for cucumber, melon, and watermelon. Agron. Sustain. Dev. 35, 251-259.

Liu, N., Yang, J., Fu, X., Zhang, L., Tang, K., Guy, K.M., Hu, Z., Guo, S., Xu, Y., Zhang, M., 2016. Genome-wide identification and comparative analysis of graftingresponsive mRNA in watermelon grafted onto bottle gourd and squash rootstocks by high-throughput sequencing. Mol. Genet. Genomics 291, 621-633.

Muramatsu, Y., 1981. Problems on vegetable grafting (In Japanese). Shisetu Engei 10,11, $48-53,46-52$.

Özarslandan, A., Sögut, M.A., Yetisir, H., Elekcioglu, I.H., 2011. Screening of bottle gourds (Lagenaria siceraria (Molina) Standley) genotypes with rootstock potential for watermelon production for resistance against Meloidogyne incognita (Kofoid and White, 1919) Chitwood and Meloidogyne javanica (Treub, 1885) Chitwood. Turkish J. Entomol. 4, 687-697.

Paris, H.S., 2015. Origin and emergence of the sweet dessert watermelon, Citrullus lanatus. Ann. Bot. 116, 133-148.

Petropoulos, S.A., Khah, E.M., Passam, H.C., 2012. Evaluation of rootstocks for watermelon grafting with reference to plant development, yield and fruit quality. Int. J. Plant Prod. 6, 481-492.

Petropoulos, S.A., Olympios, C., Ropokis, A., Vlachou, G., Ntatsi, G., Paraskevopoulos, A., Passam, H.C., 2014. Fruit volatiles, quality, and yield of watermelon as affected by grafting. J. Agric. Sci. Technol. 16, 873-885.

Rouphael, Y., Schwarz, D., Krumbein, A., Colla, G., 2010. Impact of grafting on product quality of fruit vegetables. Sci. Hortic. 127, 172-179.

Saftner, R., Luo, Y., McEvoy, J., Abbott, J.A., Vinyard, B., 2007. Quality characteristics of fresh-cut watermelon slices from non-treated and 1-methylcyclopropene- and/or ethylene-treated whole fruit. Postharvest Biol. Technol. 44, 71-79.

Sharma, R., Kaur, D., Oberoi, D.P.S., Sogi, D.S., 2008. Thermal degradation kinetics of pigments and visual color in watermelon juice. Int. J. Food Prop. 11, 439-449.

Sigüenza, C., Schochow, M., Turini, T., Ploeg, A., 2005. Use of Cucumis metuliferus as a rootstock for melon to manage Meloidogyne incognita. J. Nematol. 37, 276-80. 
Soteriou, G.A., Kyriacou, M.C., 2015. Rootstock-mediated effects on watermelon field performance and fruit quality characteristics. Int. J. Veg. Sci. 21, 344-362.

Soteriou, G.A., Kyriacou, M.C., Siomos, A.S., Gerasopoulos, D., 2014. Evolution of watermelon fruit physicochemical and phytochemical composition during ripening as affected by grafting. Food Chem. 165, 282-289.

Thies, J.A., Ariss, J.J., Hassel, R.L., Olson, S., Kousik, C.S., Levi, A., 2010. Grafting for management of southern root-knot nematode, Meloidogyne incognita, in watermelon. Plant Dis. 94, 1195-1199.

Thies, J.A., Ariss, J.J., Hassell, R.L., Buckner, S., Levi, A., 2015. Accessions of Citrullus lanatus var. citroides are valuable rootstocks for grafted watermelon in fields infested with root-knot nematodes. HortScience 50, 4-8.

Vicente-Villardon, J.L., 2014. MULTBIPLOT: a package for multivariate analysis using biplots. [WWW Document]. URL http://biplot.usal.es/classicalbiplot/ (accessed 4.1.2014).

Vinson, E.L., Woods, F.M., Kemble, J.M., Perkins-Veazie, P., Davis, A., Kessler, J.R., 2010. Use of external indicators to predict maturity of mini-watermelon fruit. HortScience 45, 1034-1037.

Yamasaki, A., Yamashita, M., Furuya, S., 1994. Mineral concentrations and cytokinin activity in the xylem exudate of grafted watermelons as affected by rootstocks and crop load. J. Japanese Soc. Hortic. Sci. 62, 817-826. 


\section{CAPÍTULO 3}

"Use of electronic nose as a phenotyping tool in watermelon: application to the evaluation of rootstock lines"

Fredes A., Cebolla-Cornejo J., Beltrán J., Gisbert C., Picó M.B., Valcárcel M., Roselló S. (Artículo pendiente de enviar a Food Chemistry).

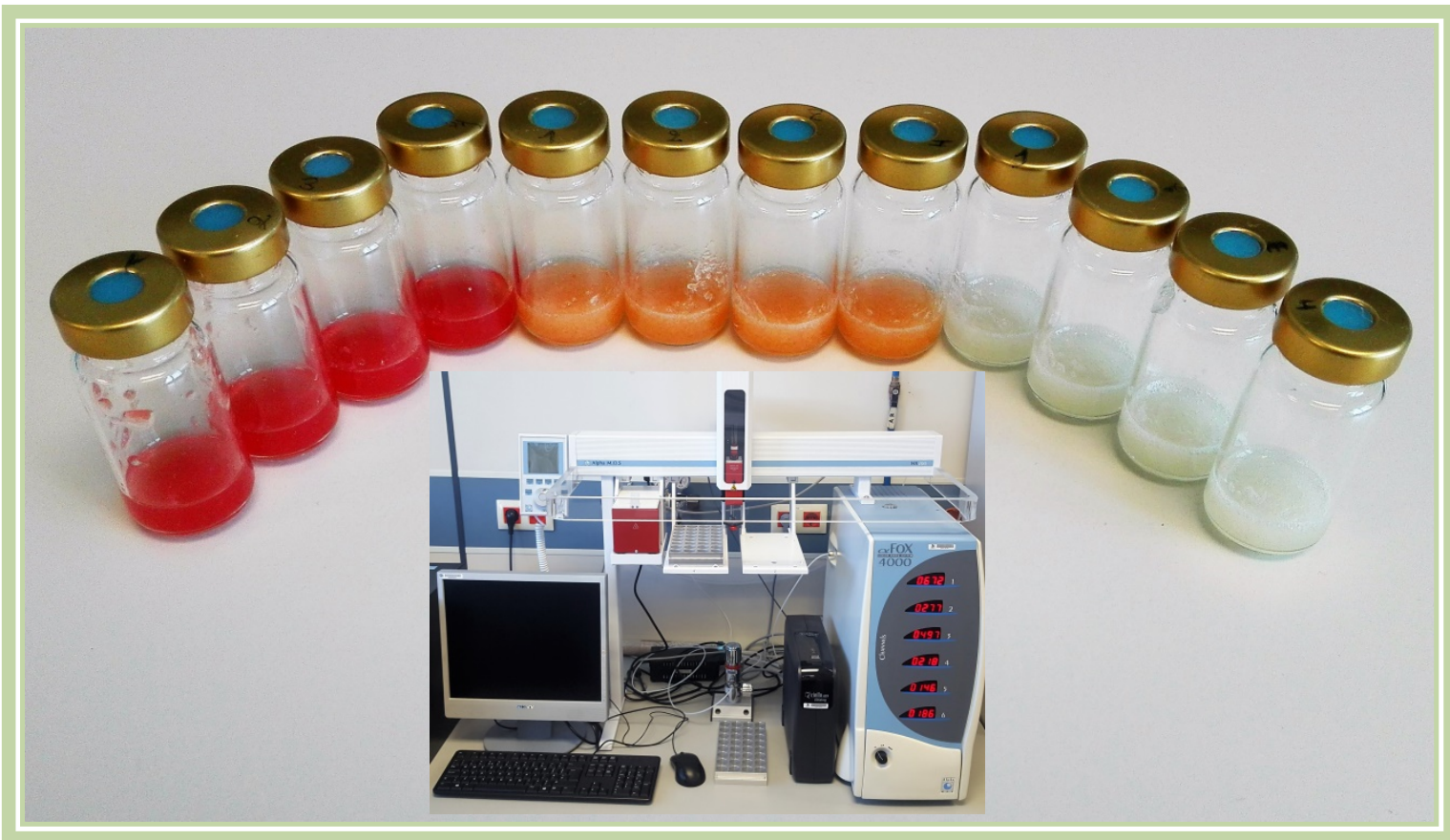





\section{RESUMEN}

La importancia que tienen los volátiles no sólo en la conformación del aroma sino también en la percepción de azúcares pone de manifiesto la necesidad de incorporar el fenotipado del perfil volátil en programas de mejora en sandía. Se ha estudiado la capacidad de un olfatómetro para evaluar estos perfiles de volátiles, aplicándolo a la evaluación de líneas de mejora de portainjertos de sandía. A partir de los resultados de respuesta máxima y pendiente de respuesta se han desarrollado mapas de similitud. Se ha comprobado que determinadas combinaciones portainjeto-cultivar pueden generar una gran variabilidad en el perfil. Los resultados obtenidos se han comparado con mapas de similitud de cuantificaciones obtenidas mediante GC-MS. El olfatómetro fue capaz de identificar no sólo qué líneas sino incluso qué muestras presentan el perfil más parecido al de la sandía sin injertar. Además, se han desarrollado modelos semi-cuantitativos que permiten obtener predicciones bastante aproximadas de los contenidos en compuestos clave como (Z)-6nonen-1-ol que pueden ser negativos en la percepción del sabor de sandía. El reducido coste y tiempo de análisis del olfatómetro electrónico y su similitud con la información proporcionada por técnicas más complejas como la GC-MS permitirá incorporar el fenotipado masivo del perfil volátil en programas de mejora, algo impensable con técnicas de cuantificación directa.

Palabras clave: Citrullus lanatus, perfiles de volátiles, calidad organoléptica, mapas de similitud 


\section{INTRODUCCIÓN}

La calidad interna de los frutos de sandía está determinada principalmente por su contenido en azúcares (glucosa, fructosa y sacarosa) (Yativ et al., 2010; Yoo et al., 2012), considerándose también muy importantes los volátiles ya que pueden intervenir en la modulación de la percepción de dulzor, al igual que ocurre en otros frutos como por ejemplo el tomate (Tieman et al., 2017).

En sandía se han descrito alrededor de 80 compuestos volátiles siendo los principales componentes de esta fracción aldehídos y alcoholes con cadenas de nueve átomos de carbono y sus ésteres derivados, encontrándose también otros compuestos como cetonas, lactonas y furanos (Beaulieu \& Lea, 2006; Pino et al., 2003; Yajima et al., 1985). En esta especie, dado el alto contenido de pigmentos carotenoides de la carne del fruto, los compuestos volátiles que se originan por degradación de estos pigmentos tienen gran importancia en la definición del aroma característico de sandías, de modo similar a como ocurre en tomate (Lewinsohn et al., 2005).

Hasta el momento el desarrollo de nuevos cultivares ha tenido como objetivo de calidad organoléptica interna la obtención de genotipos con alto contenido en azúcares, pero no se ha considerado la selección por perfiles de volátiles ya que su evaluación es muy compleja y su inclusión en los criterios de selección también. En el desarrollo de nuevos cultivares no sólo se debe considerar el genotipo del cultivar que producirá los frutos, sino también que el potencial de un determinado genotipo en la expresión de caracteres de calidad interna se ve modulado por la influencia del genotipo del portainjertos (Fredes et al., 2017; Rouphael et al., 2010) que actualmente se utilizan para prevenir problemas de ataque de patógenos del suelo (King et al., 2010) o nematodos (Liu et al., 2015; Thies et al., 2010) y mejorar la respuesta frente a condiciones de estrés (Davis et al., 2008).

Las características aromáticas de los frutos de sandía pueden evaluarse sensorialmente mediante paneles de cata entrenados. Este método proporciona resultados directos sobre el sabor y los matices aromáticos de los genotipos evaluados. Sin embargo, es un procedimiento de evaluación complejo ya que requiere el desarrollo previo de descriptores precisos con los que posteriormente se entrenará a los catadores; lento, porque no se pueden catar un gran número de muestras por sesión debido a problemas de saturación sensorial de los catadores; caro y que precisa grandes cantidades de muestra para llevarse a 
cabo. Por todo ello no resulta viable en los casos en los que se han de evaluar grandes cantidades de muestras como ocurre a lo largo de un programa de mejora. Además, se ha sugerido que en este tipo de evaluaciones los catadores pueden representar una fuente de variabilidad importante en los resultados (Causse et al., 2001). Por ello, en estas condiciones se prefiere sustituir las evaluaciones sensoriales por cuantificaciones objetivas de compuestos correlacionados con las percepciones sensoriales de calidad organoléptica interna.

En el caso de los volátiles que determinan el aroma, su evaluación suele realizarse mediante técnicas analíticas que constan de tres etapas sucesivas: la extracción de los volátiles de la muestra (concentrándolos en algunos casos), la separación de diferentes compuestos y la identificación y cuantificación. Actualmente la separación, identificación y cuantificación de compuestos volátiles se realiza en una gran diversidad de matrices de muestras principalmente mediante cromatografía de gases acoplada a espectrometría de masas (Beaulieu, 2006; Bicchi et al., 2008; Biniecka \& Caroli, 2011; Verzera et al., 2011). Las principales diferencias entre los protocolos modernos de cuantificación de volátiles se dan en la fase inicial de extracción de volátiles de la muestra. Las metodologías más utilizadas son la de espacio de cabeza dinámico mediante purga y trampa (P\&T) y las de microextracción en fase sólida (SPME).

Otro planteamiento distinto para evaluar el perfil de volátiles en diversos tipos de frutos es la utilización de olfatómetros electrónicos. Este tipo de evaluación trata de desarrollar una técnica que simule instrumentalmente la respuesta de los receptores olfativos de la nariz humana frente a la fracción volátil del fruto a evaluar. En este caso no se busca la separación y cuantificación de cada uno de los compuestos volátiles correlacionados con percepciones sensoriales. Lo que se quiere obtener es una señal electrónica producida como respuesta al estímulo conjunto de todos los volátiles de la muestra, particular y característica de cada una de ellas, y que se pueda evaluar informáticamente de forma similar a como el cerebro humano procesa las señales procedentes de los estímulos olfativos (Gardner \& Bartlett, 1999). Para ello se utilizan baterías de sensores electrónicos, en la mayor parte de los casos del tipo de óxidos metálicos (MOS), que modifican su resistencia eléctrica en función del tipo y concentración de los compuestos volátiles que los estimulan. El sistema se completa con un módulo informatizado que al aplicar diversos tipos de análisis quimiométrico pueden permitir reconocimientos de patrones de respuesta y diversos tipos de evaluación cualitativa y semi-cuantitativa por comparación con 
muestras de referencia previamente evaluadas y cuya información se ha almacenado en el sistema (Gardner \& Bartlett, 1999). El uso de narices electrónicas para la evaluación de perfiles de volátiles presenta una serie de ventajas frente a las evaluaciones sensoriales realizadas por catadores: mayor objetividad y capacidad de evaluación de un número elevado de muestras, mayor sensibilidad de discriminación y posibilidad de construir mapas escalables a largo plazo con los que evaluar y comparar perfiles de volátiles de muchas muestras evaluadas en periodos largos de tiempo. Sin embargo, también presenta una serie de limitaciones: necesidad de diversos métodos de análisis multivariante para soslayar los problemas de deriva debidos a variaciones ambientales de temperatura o humedad (Gardner \& Persaud, 2001) o de naturaleza de los sensores (Holmberg \& Artursson, 2003). A pesar de estas limitaciones son numerosos los trabajos en que se han aplicado a la clasificación de muestras de diversos tipos de frutos, tales como tomate (Hernández et al., 2008; Sinesio et al., 2000), pepino (Zawirska-Wojtasiak et al., 2009), manzana (Brezmes et al., 2000; Marrazzo et al., 2005; Young et al., 1999), pera (Zhang et al., 2008), melocotón (Benedetti et al., 2008), albaricoque (Solis-Solis et al., 2007), naranja (Di Natale et al., 2001), fresas y otros frutos rojos (Du et al., 2010; Simon et al., 1996), uva (Athamneh et al., 2008), mango (Lebrun et al., 2004), banana (Llobet et al., 1999), níspero (Guarrasi et al., 2011) y melón (Benady et al., 1995) entre otros.

En este contexto, el objetivo del presente estudio es validar el potencial de uso de un olfatómetro electrónico para realizar evaluaciones fenotípicas rápidas de perfiles de volátiles en sandía que puedan ser interesantes para ser utilizadas en la evaluación de diferentes genotipos en mejora. Se pretende utilizar las huellas electrónicas de evaluación de estas muestras no con la finalidad de clasificarlas en grupos sino para desarrollar mapas de similitud de perfiles de volátiles que permitan comparar materiales vegetales con fines de selección. Por ello se aplicará a la evaluación del efecto que tienen diferentes portainjertos en la acumulación de volátiles de un mismo cultivar comercial. Se pretende también completar la utilidad de las huellas electrónicas medidas desarrollando modelos semi-cuantitativos de predicción del contenido en algunos de los volátiles importantes en la evaluación de sandía. 


\section{MATERIALES Y MÉTODOS}

\subsection{Diseño del ensayo y condiciones de cultivo}

Se estudiaron muestras procedentes de un mismo cultivar comercial injertado sobre diferentes portainjertos. Estas muestras se evaluaron tanto mediante olfatómetro electrónico como mediante cromatografía de gases-espectrometría de masas (GC-MS), utilizando los resultados de esta segunda técnica de evaluación para validar los resultados de la primera y para sustentar modelos de predicción. El cultivar comercial de sandía utilizado fue Oneida F1 (Rijk Zwaan, Almería, España). Los portainjertos utilizados fueron: una entrada española de Citrullus lanatus var. citroides (BGV0005167) con elevada resistencia a nematodos (código $\boldsymbol{G C}$ ); una entrada de Cucurbita pepo (código GPepo), estas dos entradas proceden del Banco de Germoplasma del Instituto de Conservación y Mejora de la Agrodiversidad (COMAV, Valencia, España); dos portainjertos procedentes de hibridaciones interespecíficas de Cucurbita maxima x $C$. moschata (códigos GMM1 y GMM2), GMM1 procede del cruce de las entradas de $C$. maxima VAV 1860 (Large Warted Hubbard, Australia) y de PI 550689 de C. moschata (Canada Crookneck calabacín de USA) mientras que $\boldsymbol{G M M 2}$ es un híbrido comercializado como portainjerto (Rijk Zwaan, Almería, España). Como controles se evaluaron frutos del cultivar comercial Oneida F1 procedentes de plantas sin injertar (código $N \boldsymbol{G}$ ) y los procedentes de plantas autoinjertadas sobre el propio cultivar comercial (autoinjerto con código $\boldsymbol{S G}$ ), en este último caso para comparar el efecto de la operación de injerto sobre el desarrollo y maduración de los frutos y, por tanto, sobre el perfil de volátiles de éstos.

Se utilizó un diseño de bloques completamente al azar. Se obtuvieron muestras de 3 repeticiones por grupo de material vegetal a ensayar (cada réplica con 6 plantas). Los injertos se realizaron por el método de aproximación y un mes después todas las plantas del ensayo se trasplantaron a una parcela experimental de la compañía Rijk Zwaan, localizada en Picassent, (Valencia, España). El marco de plantación utilizado fue de $2.0 \mathrm{~m}$ x $1.0 \mathrm{~m}$ y el manejo del riego y la fertilización se hizo de acuerdo a las prácticas culturales normales para este cultivo en esa zona. Los frutos se muestrearon procedentes de todas las plantas de cada repetición cuando se observaron signos externos característicos de madurez. En cada fruto se obtuvo una porción de $5 \mathrm{~cm}$ procedente de la sección media de 
la zona ecuatorial del fruto. Para cada material vegetal, las muestras procedentes de frutos de una misma repetición se procesaron juntas para disponer de una media biológica de la repetición. Antes de proceder a la homogenización de la muestra se eliminó el pericarpio y aproximadamente $2 \mathrm{~mm}$ de carne adyacente, así como las semillas. La homogenización se realizó con una batidora de vaso (Krups KB720, Groupe Seb Iberica, Barcelona, España) y las muestras se guardaron congeladas $\left(-80^{\circ} \mathrm{C}\right)$ hasta el momento de los análisis.

\subsection{Reactivos}

Los estándares de referencia de cada uno de los compuestos volátiles estudiados (grado de pureza del 90-99,55\%) se obtuvieron de Supelco (Sigma-Aldrich y Fluka, Barcelona, España). Se prepararon soluciones madre de estándares de volátiles y a partir de ellas las mezclas de trabajo para la obtención de las curvas de calibrado en los análisis de GC-MS y para preparar el "estándar sintético" usado para la corrección de deriva (modificado de Valcárcel, 2009). La preparación, manejo y conservación de estas soluciones de estándares se realizó como se describe en Fredes et al. (2015).

Para la cuantificación de volátiles por GC-MS, los solventes utilizados para la desorción y dilución de los analitos retenidos en cartuchos Supelclean ${ }^{\mathrm{TM}}$ ENVI-Carb ${ }^{\mathrm{TM}}$ 120-400 mesh (tubos de SPE de $6 \mathrm{~mL}$ ) fueron todos de calidad GC-grade.

Como "estándar sintético" utilizado para la corrección de deriva (modificado de Valcárcel, 2009), se utilizó una mezcla de volátiles (Tabla 1) desarrollada a partir del promedio de las concentraciones de un grupo de muestras previamente analizadas por GC-MS (Fredes et al., 2015) y ajustado a los niveles de sensibilidad de la nariz electrónica. La primera dilución del estándar puro se realizó en acetona y sus respectivas diluciones con agua ultra pura (milli-Q) hasta obtener una mezcla con todos los compuestos en las concentraciones deseadas. Los viales patrón para el análisis de nariz electrónica, se prepararon mezclando $200 \mu \mathrm{L}$ de patrón sintético acuoso (10 veces más concentrados que la Tabla 1) con 1800 $\mu \mathrm{L}$ de agua destilada. 
Tabla 1. Concentraciones (ng $\left.\mathrm{mL}^{-1}\right)$ de los principales volátiles utilizados en el patrón sintético para corrección de deriva.

\begin{tabular}{|c|c|c|c|}
\hline Compuesto & Concentración & Compuesto & Concentración \\
\hline (Z)-3-Nonen-1-ol & 56,08 & Propyl butyrate & 2,75 \\
\hline 1-Hexanol & 49,44 & Phenol & 2,69 \\
\hline (E,Z)-2,6-Nonadienal & 47,30 & (E,E)-2,4-Heptadienal & 2,61 \\
\hline Hexanal & 31,38 & Eucalyptol & 2,08 \\
\hline (Z)-3-Hexen-1-ol & 24,94 & Butyl acetate & 1,95 \\
\hline Methyl-2-methyl butyrate & 24,06 & Eugenol & 1,71 \\
\hline Ethy 1-butanoate (37) & 19,76 & Beta-Ionone & 1,71 \\
\hline Nonanal & 17,13 & 1-Pentanol & 1,60 \\
\hline (Z)-6-Nonenal & 15,33 & 2-Ethylpropyl butyrate & 1,48 \\
\hline 1-Octanol & 13,97 & Amyl acetate & 1,45 \\
\hline Ethyl-2-methyl butyrate & 12,64 & 6-Methyl-5-hepten-2-one & 1,37 \\
\hline (Z)-6-Nonen-1-ol & 11,85 & Heptanal & 1,36 \\
\hline 1-Octen-3-ol & 9,89 & (E,Z)-2,6-Nonadien-1-ol & 1,33 \\
\hline Octyl acetate & 5,83 & (E,E)-2,4-Decadienal & 1,33 \\
\hline 1-Nonanol & 5,37 & Methyl hexanoate & 1,32 \\
\hline (E)-2-Heptenal & 5,00 & Beta-ciclocytral & 1,32 \\
\hline 3-Methylbutyl acetate & 4,97 & Ethyl hexanoate & 1,29 \\
\hline (E)-2-Nonenal & 4,89 & Butyl isobutyrate & 1,16 \\
\hline Octanal & 3,70 & Decanal & 0,92 \\
\hline 2-methylbutylacetate & 3,56 & Phenethyl acetate & 0,92 \\
\hline Benzaldehyde & 3,46 & (E)-2-Octenal & 0,75 \\
\hline Phenylacetaldehyde & 3,43 & Butyl butyrate & 0,74 \\
\hline 1-Decanol & 3,23 & (E,E)-2,4-Nonadienal & 0,67 \\
\hline Benzyl Alcohol & 3,23 & (E)-2-Hexenal & 0,64 \\
\hline Hexyl acetate & 3,04 & Ethyl heptanoate & 0,60 \\
\hline 2-Methyl propyl acetate & 2,77 & Benzyl acetate & 0,29 \\
\hline Geranylacetone & 2,76 & (Z)-3-Hexen-1-ol, acetate & 0,27 \\
\hline
\end{tabular}




\subsection{Determinación de compuestos volátiles por GC-MS}

La determinación de compuestos volátiles se realizó siguiendo la metodología desarrollada por Fredes et al. (2015). En este caso se analizaron 61 compuestos extraídos por purga y trampa con cartuchos de Carbowax de $500 \mathrm{mg}$ los cuales fueron previamente acondicionados con $5 \mathrm{~mL}$ de dietil éter y $5 \mathrm{~mL}$ de $\mathrm{n}$-hexano. Para la extracción se dispusieron $30 \mathrm{~g}$ de muestra en un matraz de $150 \mathrm{~mL}$ cerrando el sistema con un tapón esmerilado provisto de una entrada de nitrógeno gas y una salida conectada a la trampa de volátiles. En la extracción las muestras se homogeneizaron a $300 \mathrm{rpm}$ y una temperatura de $40{ }^{\circ} \mathrm{C}$; el flujo del gas nitrógeno fue de $1.6 \mathrm{~L} \mathrm{~min}^{-1}$. Pasados 49 minutos de extracción, se eluyeron los compuestos retenidos con $5 \mathrm{~mL}$ de mezcla dietil éter: hexano (1:1) y $5 \mathrm{~mL}$ de dietileter hacia un tubo graduado. Posteriormente se procedió a concentrar el extracto bajo flujo de gas nitrógeno en un baño de agua a $35^{\circ} \mathrm{C}$, hasta un volumen de $0.5 \mathrm{~mL}$.

El análisis de compuestos volátiles se llevó a cabo en un cromatógrafo de gases (Varian CP-3800) acoplado a un espectrómetro de masas (trampa iónica, Saturn 4000, Varian) donde se inyectó $1 \mu \mathrm{L}$ de muestra en modo splitless (temperatura de inyector $220^{\circ} \mathrm{C}$ ). Para la separación se utilizó una columna capilar (Supelco, Bellefonte, PA) de 30m x 0.25mm Supelcowax $10(0.25 \mu \mathrm{m}$ diámetro interno). El gas portador fue helio con un flujo de $1 \mathrm{~mL}$ $\min ^{-1}$. Se estableció el siguiente gradiente de temperatura: $40{ }^{\circ} \mathrm{C}$ durante $5 \mathrm{~min}$; incremento de $4{ }^{\circ} \mathrm{C} \mathrm{min}{ }^{-1}$ hasta $160{ }^{\circ} \mathrm{C}$; finalmente un incremento de $30{ }^{\circ} \mathrm{C} \mathrm{min}-1$ hasta $250{ }^{\circ} \mathrm{C}$, temperatura que se mantuvo durante $1 \mathrm{~min}$, con un tiempo total de análisis de $39 \mathrm{~min}$. Los espectros fueron obtenidos en modo full-scan (rango de m/z 50-200 Da) usando ionización de impacto electrónico $(70 \mathrm{eV})$ en modo positivo y configuración de ionización externa. La temperatura de la interfase GC-MS fue de $275{ }^{\circ} \mathrm{C}$, de la trampa de iones $190{ }^{\circ} \mathrm{C}$ y del manifold $60^{\circ} \mathrm{C}$. La identificación se realizó a partir de los estándares por comparación de sus espectros de masa, tiempos de retención de los picos y sus índices de retención calculados según la fórmula utilizada por Kovats (1958) a partir de una mezcla de nalcanos $\left(\mathrm{C}_{7}-\mathrm{C}_{30}\right)$ en las mismas condiciones de análisis. Para la cuantificación se utilizaron curvas externas de calibrado en solvente para todos los compuestos estudiados. 


\subsection{Sistema de nariz electrónica}

Se utilizó un olfatómetro electrónico comercial modelo Fox 4000 (Alpha M.O.S., Toulouse, France) que consta de 18 sensores, de óxidos metálicos semiconductores (MOS, Metal Oxide Semiconductors) dispuestos en tres cámaras conectadas en serie, un sistema de inyección automatizado (CombiPAL HS100, CTC Anlytics, Zwingen, Switzerland) y un programa de ordenador (AlphaSoft v9) para el control y obtención de los datos. Los sensores del equipo son de tres tipos: 6 del tipo LY2 (LY2/LG, LY2/G, LY2/AA, LY2/GH, LY2/gCTl, LY2/gCT) basados en $\mathrm{Cr}_{2} \mathrm{O}_{3}$ y Titanio, 5 del tipo $\mathbf{T}$ (T30/1, T40/2, T40/1, T70/2, TA/2) y 7 del tipo $\mathbf{P}$ (P10/1, P10/2, P40/1, PA/2, P30/1, P40/2, P30/2) ambos grupos basados en $\mathrm{SnO}_{2}$ y $\mathrm{Pt} / \mathrm{Pd}$.

Para realizar el análisis se pesaron 2 gramos de muestra de sandía triturada y se dispusieron en viales de $10 \mathrm{~mL}$ que se sellaron herméticamente. En el caso del estándar sintético se utilizaron $2 \mathrm{~mL}$ se solución en cada vial. Los viales se incubaron durante 10 minutos a 45 ${ }^{\circ} \mathrm{C}$ en agitación (500 rpm). De cada vial se extrajo un volumen de $2 \mathrm{~mL}$ del espacio de cabeza generado utilizando una jeringa de $2.5 \mathrm{~mL}$ acondicionada a $55^{\circ} \mathrm{C}$. Posteriormente se inyectó en las cámaras de los sensores electrónicos. El tiempo de limpieza de la jeringa fue de 120 segundos (s) con una velocidad de flujo de $500 \mu \mathrm{L} \mathrm{s}^{-1}$. El tiempo de cada adquisición de señal fue de $120 \mathrm{~s}$ con períodos de lectura repetidos cada $1 \mathrm{~s}$. Cada muestra se analizó por duplicado, realizando secuencias de 16 viales ( 6 muestras por secuencia) con 4 patrones sintéticos colocados al azar para la posterior corrección de deriva. Como gas de arrastre, el equipo utilizó aire limpio y seco (sin trazas de humedad) a un flujo de $150 \mathrm{~mL}$ $\min ^{-1}$. El equipo se calibró una vez por semana según las especificaciones del fabricante.

La respuesta de los sensores se midió como la variación de la resistencia eléctrica debida a la reacción de los compuestos volátiles de la muestra sobre la superficie activa del sensor. Estas respuestas presentan un aumento rápido en la señal con el tiempo (crecimiento sigmoide) hasta un punto donde se obtiene la intensidad máxima de respuesta y donde se produce un equilibrio dinámico (igual velocidad de adsorción y desorción de los volátiles en la superficie de sensor). El tiempo en el que se alcanza esta respuesta máxima depende de las características fisicoquímicas de cada sensor y del estímulo recibido. A partir de la representación gráfica de la respuesta de cada sensor frente a los estímulos de volátiles se obtuvieron dos tipos de parámetros: la intensidad máxima normalizada de cada sensor $\left(I N_{\text {máx}}\right)$ calculada como $\left(\mathrm{R}_{\mathrm{i}}-\mathrm{R}_{\text {máx }}\right) / \mathrm{R}_{\mathrm{i}}$ (donde $\mathrm{R}_{\mathrm{i}}$ es la resistencia eléctrica de los sensores 
cuando detectan aire limpio y $\mathrm{R}_{\text {máx }}$ es la resistencia electricidad durante la detección de los compuestos), y la pendiente (K) calculada según la fórmula (Lin \& Zhang, 2016): $K=\frac{t_{\text {máx }}-t_{0}}{I N_{\text {máx }}}$ y que también estaría relacionada con el proceso de absorción de volátiles en la superficie del cada sensor (García-González \& Aparicio, 2002).

La corrección de deriva se realizó según lo expuesto por Valcárcel (2009) con ligeras modificaciones. Básicamente consiste en hacer una medida periódica de estándares de referencia que permiten cuantificar la variación (deriva) en la respuesta de los sensores con el tiempo. Una vez obtenida esta variación, se aplica un cálculo que elimina la parte de señal que no corresponde a la muestra propiamente dicha. El cálculo realizado fue el siguiente:

$S_{\text {medida }}^{0}=\left[\hat{S}_{\text {medida }}\left(1-\varepsilon_{\text {deriva }}(t)\right)\right]+S_{\text {medida }}$, donde $S_{\text {medida }}^{0}$ es la señal medida corregida, $\hat{S}_{\text {medida }}$ es la media de todas las repeticiones correspondientes a una muestra, $\varepsilon_{\text {deriva }}(t)$ es la función de la deriva y $S_{\text {medida }}$ es el valor de medida dado por el instrumento.

\subsection{Análisis de datos}

Para facilitar el estudio de los perfiles de volátiles que los diferentes portainjertos condicionan en el cultivar comercial Oneida F1 se utilizó una metodología de reducción de dimensionalidad. Se trata de recoger el máximo de la variabilidad existente y proyectarla en un subespacio de variables latentes para mostrar las similitudes entre diferentes materiales como una función inversa de su distancia en el plano de proyección. Esta representación gráfica permite además un estudio de clasificación de las diferentes muestras en los grupos estudiados (Wise et al., 2006). Se ha optado además por el análisis discriminante basado en la regresión parcial por mínimos cuadrados (PLS-DA) porque las nuevas variables discriminantes latentes que calcula son ortogonales entre sí por lo que se consigue evitar problemas de colinealidad que, por la naturaleza de las variables originales (grupos de compuestos volátiles que comparten naturaleza química y rutas biosintéticas de origen y grupos de sensores con similitudes de materiales de composición) pueden darse si se utilizan otros tipos de análisis como pueden ser los análisis discriminantes lineales (LDA) (Hubert \& Vanden Branden, 2003). Para el adecuado desarrollo de los modelos, los datos en bruto se pre-trataron con el método de autoescalado para evitar que las diferencias de escala de algunas variables sesguen la modelización. La validación de los modelos de 
reducción de dimensionalidad y clasificación obtenidos se ha hecho por medio del método de validación cruzada (CV) de tipo ventana veneciana (venetian blinds) buscando disminuir los errores mínimo cuadráticos de validación cruzada (RMSECV) y encontrar un número óptimo de variables latentes evitando problemas de sobreajuste de los modelos (Brereton \& Lloyd, 2014). Para ello, se ha seguido el criterio de dejar de incluir variables latentes cuando la reducción de RMSECV es inferior al $2 \%$ intentando en todo momento utilizar el menor número posible de estas. Dado que estos modelos pueden estar muy condicionados por la presencia de valores anómalos estos se han eliminado cuando sobrepasaban los umbrales de confianza de los valores de los estadísticos $\mathrm{T}^{2}$ de Hotelling y los residuos Q (Jackson \& Mudholkar, 1979).

El valor de RMSECV se calculó según la siguiente ecuación:

$\boldsymbol{R M S E C V}=\sqrt{\frac{\sum_{i=1}^{n}\left(\hat{y}_{c v i}-y_{i}\right)^{2}}{n}}$, donde $y_{i}$ son los valores medidos e $\hat{y}_{c v i}$ los calculados por el modelo para los subgrupos de validación cruzada.

Los valores de $\mathrm{T}^{2}$ de Hotelling y los residuos $\mathrm{Q}$, se obtienen a partir de las siguientes fórmulas: $\quad \boldsymbol{T}_{i}^{2}=t_{i} \lambda^{-1} t_{i}^{T}=x_{i} P_{k} \lambda^{-1} P_{k}^{T} x_{i}^{T}$

$\boldsymbol{Q}_{i}=e_{i} e_{i}^{T}=x_{i}\left(1-P_{k} P_{k}^{T}\right) x_{i}^{T}$

Donde $x_{i}$ es la i-ésima fila de la matriz de $m$ muestras x $n$ compuestos volátiles, $P_{k}$ es la késima columna de la matriz de coordenadas de los compuestos volátiles referidas a las variables latentes utilizadas en el modelo PLS, $\lambda$ la matriz diagonal que contiene los valores propios $\left(\lambda_{1}\right.$ a $\left.\lambda_{k}\right)$ correspondientes a los $k$ vectores propios (variables latentes, LVs) utilizados en el modelo, $t_{i}$ la i-ésima fila de la matriz $T_{k}$ con los $\mathrm{k}$ vectores de coordenadas de las muestras referidas a las variables latentes retenidas en el modelo, $e_{i}$ la i-ésima fila de la matriz $E$ de residuos e $I$ la matriz identidad $(n \times n)$.

La bondad de la clasificación se determinará por medio del porcentaje de aciertos de cada una de las clases basándose en los valores de especificidad (número de muestras predichas fuera de la clase dividido el número de muestras que deberían estar fuera de la clase) y sensibilidad (número de muestras predichas en la clase divido el número de muestras de la clase). La sensibilidad y especificidad se obtiene para cada una de las clases tanto para los resultados del calibrado (auto-predicción) como para los de la validación cruzada. El porcentaje de aciertos de cada clase se determina como una media ponderada de sus valores de especificidad y sensibilidad (Wise et al., 2006). 
Los modelos de predicción del contenido en compuestos volátiles a partir de parámetros de señal electrónica obtenida con sensores se han desarrollado utilizando la técnica de regresión parcial mínimo cuadrática (PLS). Los criterios de pre-tratamiento, validación de modelos, selección del número de variables latentes y eliminación de datos anómalos han sido similares a los utilizados en los análisis PLS-DA. Para afinar los modelos obtenidos se ha utilizado una metodología jerárquica de selección de variables originales a considerar en el modelo definitivo (iPLS) tanto con la opción incremental (inclusión secuencial de variables) como con la opción detrimental (exclusión secuencial de variables) seleccionando en cada caso la opción que proporcionó modelos con menor error mínimo cuadráticos de validación cruzada (RMSECV) (Wise et al., 2006).

Todos los cálculos descritos se han realizado con el módulo PLS Toolbox versión 8.2.1 (Eigenvector Research Inc, Wenatchee, WA, USA) en entorno MATLAB (versión R2014a, 8.3.0.532, MathWorks, Inc., Natick, MA, USA).

\section{RESULTADOS Y DISCUSIÓN}

La composición de volátiles de las muestras estudiadas (Figura 1) muestran, en general, muchas similitudes, aunque los distintos pies inducen diferencias en los volátiles acumulados, que resultan importantes desde el punto de vista de la utilización de diferentes portainjertos, ya que se ha demostrado que influyen en características de calidad organoléptica de los frutos (Fredes et al., 2017). De los 61 volátiles analizados, 30 de ellos presentaron contenidos superiores al límite de cuantificación, siendo los niveles de los volátiles cuantificados coherentes con los encontrados por otros investigadores que realizaron cuantificaciones o semi-cuantificaciones de volátiles en esta matriz (Lewinsohn et al. 2005; Beaulieu, 2005; Liu et al., 2012; Petropoulos et al., 2014). No obstante, los contenidos de los diferentes volátiles evaluados están muy condicionados tanto por el material vegetal considerado, el ambiente de cultivo y la metodología de evaluación utilizada. 

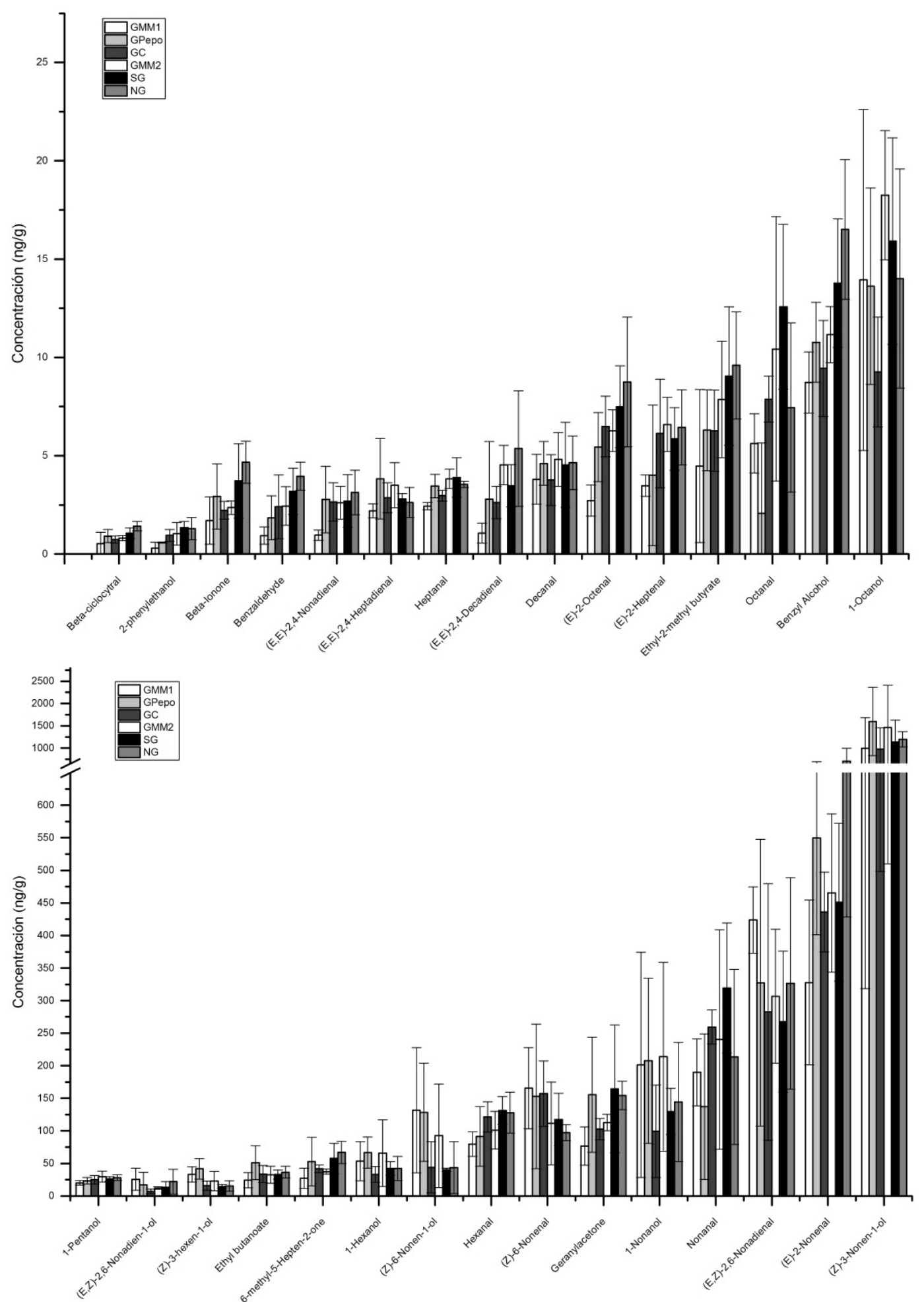

Figura 1. Perfil de volátiles (promedio \pm desviación estándar expresados en $\mathrm{ng} \mathrm{g}^{-1}$ ) para frutos de sandía (Citrullus lanatus F1 Oneida) sin injertar $(\boldsymbol{N G})$, autoinjertados $(\boldsymbol{S G})$ e injertados en: patrón experimental de Cucurbita (C. maxima x C. moschata) híbrido F1 (GMM1), híbrido comercial F1 de Cucurbita (C. maxima x C. moschata) Cobalt (GMM2), patrón de Cucurbita (C. pepo) (GPepo) y patrón experimental de melón citroides (Citrullus lanatus var. citroides) (GC). 
Con los resultados de cuantificación de volátiles por cromatografía de gases-espectrometría de masas en las diferentes combinaciones de portainjerto con Oneida F1 se realizó un análisis de clasificación discriminante a partir de regresiones parciales mínimo cuadráticas (PLS-DA) (Tabla 2A y Figura 2A). En nuestro caso de las 30 variables originales (volátiles cuantificados) se pasa a 2 variables discriminantes subyacentes obtenidas por combinación lineal de los contenidos en volátiles determinados inicialmente. Esta reducción de dimensionalidad a dos variables latentes discriminantes explica el 55,77\% de la variabilidad para el contenido en volátiles y el 21,76\% de la agrupación del diseño. La explicación de la variabilidad presente puede considerarse suficiente (supera el 50\%) considerando que los resultados procedentes de la cuantificación de volátiles son bastante variables debido posiblemente a la complejidad del proceso de extracción y cuantificación así como a la dificultad de integración de los picos (Young et al., 1999). Sin embargo, la explicación de la agrupación de las muestras no es elevada. Esto se refleja también en que los resultados de discriminación de grupos de este análisis muestran que los errores de clasificación oscilaron entre el 7 y el 33\%, siendo la sensibilidad (proporción de muestras del grupo clasificadas correctamente dentro del grupo) del modelo de clasificación absoluta (1) en todas las clases excepto cuando se utiliza como portainjerto C. pepo (Tabla 2A) y la especificidad (proporción de muestras de otros grupos incluidas incorrectamente dentro del grupo), algo más baja, especialmente cuando se utiliza como portainjerto el híbrido interespecífico Cobalt (C. maxima x C. moschata). Sin embargo, cuando se aplica remuestreo (validación cruzada) para comprobar la influencia de la variabilidad intra-grupo en los resultados de clasificación estos no son tan satisfactorios (Tabla 2A). El que los resultados de clasificación con la validación cruzada empeoren con respecto a los que se obtienen con el modelo de calibrado inicial suele suceder cuando la variabilidad dentro de grupo es elevada (Wise, 2006). 
Tabla 2. Modelos de clasificación (calibrado y validaciones cruzadas) obtenidos por PLSDA de las muestras analizadas por GC-MS (A) y E-Nose (B). Donde los valores de sensibilidad y especificidad van desde 0 (mínimo) a 1 (máximo).

\begin{tabular}{|c|c|c|c|c|c|c|c|}
\hline $\mathbf{A}$ & & & & & & & \\
\hline \multirow[t]{3}{*}{ Sensibilidad } & & GMM1 & GMM2 & GPepo & $G C$ & $S G$ & $N G$ \\
\hline & Cal & 1,00 & 1,00 & 0,67 & 1,00 & 1,00 & 1,00 \\
\hline & $\mathrm{CV}$ & 0,67 & 0,00 & 0,33 & 0,67 & 0,67 & 0,33 \\
\hline \multirow[t]{3}{*}{ Especificidad } & & GMM1 & GMM2 & GPepo & $G C$ & $S G$ & $N G$ \\
\hline & $\mathrm{Cal}$ & 0,87 & 0,33 & 0,87 & 0,53 & 0,60 & 0,87 \\
\hline & $\mathrm{CV}$ & 0,87 & 0,60 & 0,87 & 0,60 & 0,60 & 0,73 \\
\hline \multirow[t]{3}{*}{ \%Error Clasif. } & & GMM1 & GMM2 & GPepo & $G C$ & $S G$ & $N G$ \\
\hline & $\mathrm{Cal}$ & 7 & 33 & 23 & 23 & 20 & 7 \\
\hline & $\mathrm{CV}$ & 23 & 70 & 40 & 37 & 37 & 47 \\
\hline
\end{tabular}

\begin{tabular}{|c|c|c|c|c|c|c|c|}
\hline \multicolumn{8}{|l|}{ B } \\
\hline Sensibilidad & & GMM1 & GMM2 & GPepo & $G C$ & $S G$ & $N G$ \\
\hline & $\mathrm{Cal}$ & 1,00 & 0,67 & 0,67 & 1,00 & 1,00 & 1,00 \\
\hline & C.V. & 0,33 & 0,00 & 0,67 & 0,67 & 0,67 & 0,33 \\
\hline \multirow[t]{3}{*}{ Especificidad } & & GMM1 & GMM2 & GPepo & $G C$ & $S G$ & $N G$ \\
\hline & Cal & 0,87 & 0,73 & 0,73 & 0,47 & 0,80 & 0,73 \\
\hline & C.V. & 0,80 & 0,80 & 0,73 & 0,47 & 0,73 & 0,80 \\
\hline \multirow[t]{3}{*}{ \%Error Clasif. } & & GMM1 & GMM2 & GPеро & $G C$ & $S G$ & $N G$ \\
\hline & Cal & 7 & 30 & 30 & 27 & 10 & 13 \\
\hline & C.V. & 43 & 60 & 30 & 43 & 30 & 43 \\
\hline
\end{tabular}

Estos valores más bajos de sensibilidad obtenidos indican que, aunque se observa que los diferentes portainjertos condicionan características distintivas en el perfil de volátiles del cultivar injertado (separación entre grupos bastante clara en la Figura 2A) existe, en general, una amplia variabilidad intra-grupo y, dado que estamos evaluando combinaciones de materiales vegetales en los que la parte aérea injertada es la misma para todos, es inevitable que se den algunos solapes en los perfiles de volátiles de algunos de los materiales vegetales estudiados (Figura 2A). 

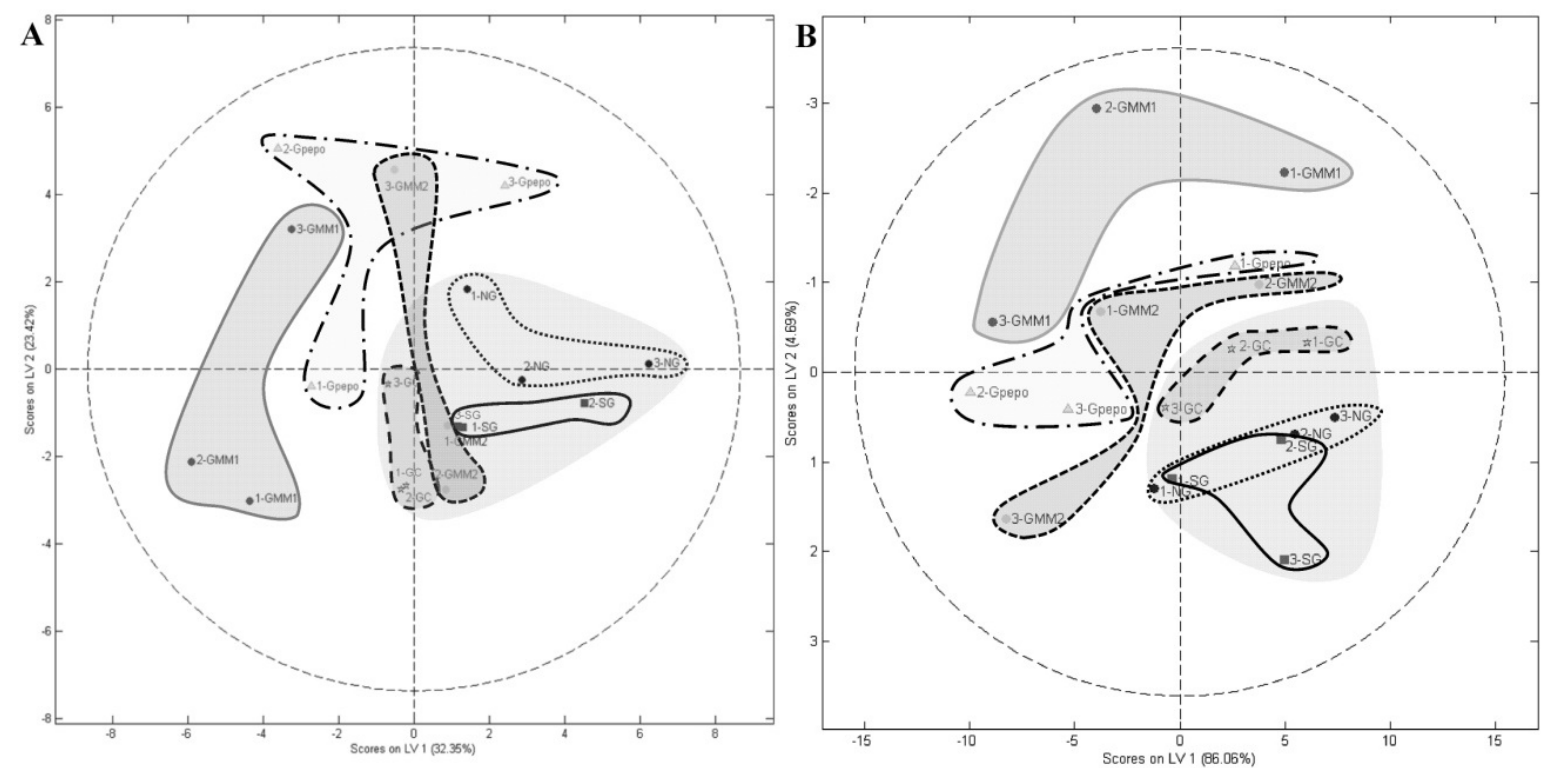

Figura 2. Mapas de similitud obtenidos por PLS-DA de los datos de perfil volátil obtenidos por cromatografía (A) y nariz electrónica (B) a partir de frutos de sandía (Citrullus lanatus F1 Oneida) sin injertar $(\boldsymbol{N G})$, autoinjertados $(\boldsymbol{S} \boldsymbol{G})$ e injertados en: patrón experimental de Cucurbita (C. maxima x C. moschata) híbrido F1 (GMM1), híbrido comercial F1 de Cucurbita (C. maxima x C. moschata) Cobalt (GMM2), patrón de Cucurbita (C. pepo) (GPepo) y patrón experimental de melón citroides (Citrullus lanatus var. citroides) $(\boldsymbol{G C})$.

Esta amplia variabilidad intra-grupo no es rara cuando se evalúan perfiles de volátiles en frutos. Por ejemplo, en fresones se observó que las diferencias genéticas entre material vegetal y la influencia de la conservación frigorífica podrían originar coeficientes de variación en el contenido de los volátiles evaluados cercanos al $20 \%$ en muchos casos y en algunos superiores al 40\% (Douillard \& Guichard, 1990). En melón y sandía, está variabilidad intra-grupo puede verse incrementada por la dificultad de muestrear frutos en el mismo estado de madurez (Abbaszadeh et al., 2015; Vinson et al., 2010) y a que los signos externos de madurez no permiten determinar ni siquiera aproximadamente si el proceso de acumulación de volátiles se halla muy avanzado.

No obstante, desde el punto de vista de evaluación de material vegetal, más que la bondad de clasificación, que depende en gran medida tanto de la variabilidad dentro del grupo como de las diferencias entre grupos, resulta mucho más interesante poder estudiar las 
similitudes o diferencias no solo entre los diferentes materiales vegetales que forman los grupos, sino también entre las muestras de un mismo grupo ya que esto permite evaluar de forma más amplia las características del material vegetal. Es decir, en nuestro caso es muy interesante disponer de "mapas" en los que las distancias entre las proyecciones de los puntos muestren lo mejor posible las relaciones y agrupaciones entre los diferentes materiales estudiados debidas a sus perfiles de volátiles, así como la homogeneidad de las muestras de un mismo grupo (variabilidad intra-grupo). En este sentido las metodologías de reducción de dimensionalidad (PCA, PLS-DA, biplots) que permiten una representación gráfica de la variabilidad existente en un subespacio reducido facilitan la interpretación de los resultados (Krzanowski, 2000) y con ello la evaluación del material vegetal con fines de selección.

Por ello, a pesar de la variabilidad intragrupo presente en las muestras estudiadas, el uso de "mapas de similitud" de perfiles de volátiles (Figura 2A) permite constatar que no hay diferencias importantes en los materiales vegetales cuyos portainjertos son de sandía: las sandías no injertadas $(\boldsymbol{N G})$ y las autoinjertadas $(\boldsymbol{S G})$ tiene perfiles muy parecidos que se observan próximos en el mapa. A estos materiales también se aproxima el perfil del cultivar injertado sobre un ancestro de sandía $(\boldsymbol{G C})$. Los materiales injertados sobre portainjertos de otras especies diferentes a la sandía muestran, en general, un perfil más alejado de los anteriores. Este es el caso de los materiales injertados sobre C. pepo (GPepo) y sobre el híbrido interespecífico GMM1 de C. maxima x C. moschata. El híbrido interespecífico GMM2 muestra un comportamiento más variable y más complejo de explicar. En general, las características de los perfiles de volátiles de los materiales injertados sobre portainjertos de sandía o de los híbridos interespecíficos de calabaza son coherentes con los mostrados en un trabajo anterior (Fredes et al., 2017). Se constata, por tanto, que esta metodología de evaluación permite una caracterización amplia del material vegetal que puede ser muy útil en mejora.

Por otro lado, estos mismos análisis discriminantes mediante PLS-DA realizados con los resultados obtenidos al evaluar las huellas electrónicas de volátiles con la nariz electrónica (Tabla 2B y Figura 2B) redujeron la dimensionalidad de 36 variables iniciales (18 valores de respuesta máxima normalizada y 18 de pendiente) a dos variables latentes discriminantes siendo claramente mejor el porcentaje de variabilidad de la respuesta de los sensores frente al estímulo del conjunto de volátiles $(94,15 \%)$ que en el caso del PLS-DA realizado a partir de los datos de cromatografía y muy similar para la explicación de la 
variabilidad de los grupos de material vegetal estudiado (20,91\%). En este caso, tanto la bondad de ajuste del modelo de clasificación (Tabla 2B) como la caracterización de respuestas frente a perfiles de volátiles y variabilidad intra-grupo (Figura 2B) ha sido similar a la obtenida con los datos de cromatografía de gases-espectrometría de masas. Por lo que puede realizarse este tipo de caracterización con cualquiera de los dos métodos de evaluación indistintamente. La similitud de ambos análisis va más allá de las tendencias generales, ya que al igual que en el caso de la cromatografía, con nariz electrónica se observó un mayor nivel de variación en las muestras correspondientes a los patrones GMM2, GPepo y GMM1. De hecho, al igual que ocurrió con los datos de cromatografía las muestras 3-GMM2, 2-GPepo y 3-GPepo y 3-GMM1 se agrupan muy próximas entre sí. También de nuevo 3-GMM1 presenta un perfil distanciado del resto de muestras de GMM1 y lo mismo ocurre de nuevo con las muestras 3-GMM2 y 1-GPepo que no se agrupan cerca del resto de su grupo. Con los dos métodos de evaluación se reproduce de forma muy fidedigna el patrón de variación de los materiales vegetales estudiados por lo que ambos pueden ser utilizados para este tipo de fenotipado. Con ello se amplía el tipo de aplicaciones en las que se utilizan los olfatómetros electrónicos para la evaluación de perfiles de volátiles, ya que en la mayor parte de los casos se han utilizado para, a partir de los grupos de muestras en estudio y los modelos de discriminación desarrollados con ellas, predecir la pertenencia de otras muestras problema a alguno de los grupos establecidos (Brezmes \& Llobet, 2016; Yang et al., 2015).

Una vez comprobada la elevada relación entre los resultados de nariz electrónica y cromatografía, se exploraron las posibilidades de desarrollar modelos de predicción de la acumulación de compuestos volátiles individuales, a partir de parámetros de señal electrónica obtenida con los sensores del olfatómetro. Para ello, se emplearon regresiones parciales mínimo cuadráticas (PLS) seguidas de selecciones jerárquicas de las variables utilizadas en los modelos (i-PLS) para escoger los parámetros electrónicos que mejor permiten predecir los niveles de cada volátil en particular, y mejorar los ajustes de los modelos a los resultados de evaluación obtenidos. Los modelos de predicción del contenido de los compuestos (Z)-6-nonen-1-ol, geranylacetone, (Z)-6-nonenal, 1-hexanol y heptanal fueron especialmente buenos (Tabla 3), presentando coeficientes de regresión para los modelos lineales de calibración por encima de 0.89. Para estos mismos compuestos, los modelos de validación cruzada presentaron también un buen ajuste, con coeficientes de regresión lineal superiores a 0.72, así como errores de validación cruzada (RMSECV) bajos. En general, los errores de validación cruzada para estos modelos están 
comprendidos entre un 10 y un $17 \%$ del valor máximo del contenido detectado para estos compuestos en los frutos de sandía, por lo que a efectos de predicción para una primera preselección pueden ser válidos. No obstante, hay que tener en cuenta que, para disponer de modelos robustos y más generales, aún se debe continuar con este tipo de trabajos incrementando el número de genotipos evaluados y los ambientes y métodos de cultivo en que se han obtenido para que cubran un gran número de casos y por ello sean más generales.

Por otra parte, los compuestos (Z)-3-nonen-1-ol, ethyl butanoate, (Z)-3-hexen-1-ol, 1nonanol, hexanal, 6-methyl-5-hepten-2-one, (E,E)-2,4-heptadienal, decanal y (E,Z)-2,6nonadienal presentaron valores de $\mathrm{R}^{2}$ entre 0.70 - 0.88 en calibración y $0.41-0.85$ en validación cruzada (Tabla 3). Estos modelos, menos precisos que los anteriores podrían utilizarse como modelos semi-cuantitativos exploratorios para dichos compuestos. En el resto de casos los modelos con presentaron valores de $\mathrm{R}^{2}$ de calibración inferiores a $0.75 \mathrm{y}$ de $\mathrm{R}^{2}$ de validación inferiores a 0.47 (Tabla 4). Es posible que el menor ajuste de estos modelos de predicción se deba a que los sensores electrónicos utilizados son menos sensibles a algunos compuestos (Song et al., 2010), por lo que no sería prudente su utilización para predicción ya que los errores de predicción que se cometen con ellos serían demasiado elevados.

Estos resultados son bastante similares a los obtenidos por otros autores que también han aplicado esta metodología para la evaluación de volátiles en otros cultivos (Lin \& Zhang, 2016) lo que corrobora las posibilidades de uso de esta estrategia de evaluación para complementar las comparaciones entre genotipos con predicciones del contenido de 14 compuestos, algunos de ellos considerados como importantes y característicos del perfil de volátil de sandía (Beaulieu \& Lea, 2006; Xisto et al., 2012). También puede resultar interesante para la selección negativa de genotipos con características detrimentales de calidad o en procesos de control de calidad para detectar partidas fuera de tipo. En este contexto se incluyen las predicciones de (Z)-6-nonen-1-ol porque es un compuesto cuya acumulación está muy condicionada por el tipo de portainjerto utilizado en el cultivo apareciendo en mayor cantidad al usar portainjertos híbridos de Cucurbita (Condurso et al., 2012; Fredes et al., 2016), siendo su presencia negativa, ya que las notas aromáticas características de calabaza que provoca se asocian a sandía sobremadura poco apreciada por el consumidor (Saftner et al., 2007). Por otro lado, Z-6-nonenal y E,Z-2,6-nonadienal pueden ser abundantes en sandía sin pepitas y se asocian a notas aromáticas características 
de melón y pepino (Beaulieu et al., 2006) que en las evaluaciones de sandía no son bien valoradas (Petropoulos et al., 2014).

\section{CONCLUSIONES}

En este estudio se ha demostrado que el uso de nariz electrónica permite una evaluación fenotípica rápida de los perfiles de volátiles de genotipos de sandía. Esta metodología no precisa laboriosas y lentas preparaciones de muestra como ocurre en otras evaluaciones analíticas mediante técnicas mucho más caras y tediosas como con algunas de las modalidades de cromatografía de gases acoplada a espectrometría de masas (GC-MS) más ampliamente utilizadas, por lo que puede ser muy interesante en la evaluación de grandes cantidades de genotipos de sandía. La evaluación mediante nariz electrónica permite construir mapas de similitud de muestras por características de perfil de volátiles que ponen de manifiesto tanto las diferencias entre grupos de material vegetal como la variabilidad dentro un mismo grupo de forma similar a como se obtiene con datos de cuantificación de volátiles mediante GC-MS, siendo en algunos casos más precisa que esta al caracterizar la variabilidad. Por tanto, es una metodología de fenotipado rápido muy interesante para ser utilizada en programas de mejora de la calidad en sandía. Como resultado adicional, se han presentado evidencias de que se pueden desarrollar buenos modelos de predicción de contenido en 14 compuestos volátiles importantes en el perfil de volátiles de sandía que completarían los análisis de evaluación de las tendencias de variabilidad y comparación de material vegetal. Si bien es cierto que se necesitan más estudios de este tipo para disponer de modelos de predicción más robustos y generales. 
Tabla 3. Modelos con buena predicción ${ }^{\mathrm{a}}$ de contenidos de volátiles (ng $\mathrm{g}^{-1}$ ) a partir de respuestas de los sensores de nariz electrónica.

\begin{tabular}{|c|c|c|c|c|c|c|c|c|c|c|c|c|c|c|}
\hline & 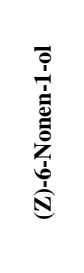 & 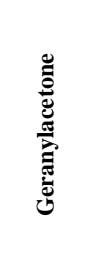 & 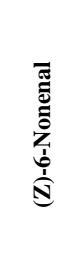 & $\begin{array}{l}\bar{\Xi} \\
\\
\text { : }\end{array}$ & 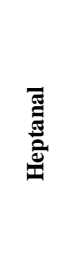 & 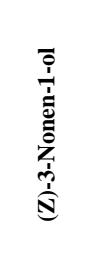 & 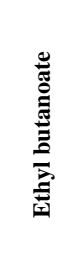 & 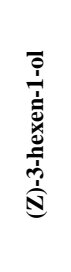 & 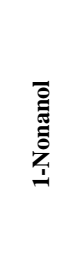 & 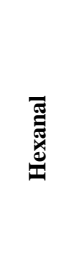 & 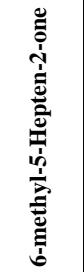 & 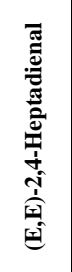 & 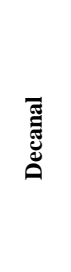 & 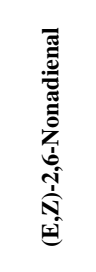 \\
\hline Bo & 632,9 & $-18,2$ & $-3069,6$ & $-227,4$ & $-75,1$ & $-13854,0$ & $-493,5$ & 710,5 & $-1654,7$ & $-1297,4$ & $-1583,8$ & $-90,8$ & 36,6 & $-2515,6$ \\
\hline INm1(LY2/LG) & 275,3 & $-3701,9$ & 2644,5 & $-293,1$ & $-46,2$ & $-12341,7$ & $-459,5$ & & & $-299,5$ & $-602,8$ & & & 3692,3 \\
\hline INm2(LY2/G) & & & 170,8 & & 4,2 & & & & & & & & $-0,4$ & \\
\hline INm3(LY2/AA) & $-34,0$ & & 141,0 & & & & & & & & & & & \\
\hline INm4(LY2/GH) & & & & & 2,7 & & & $-68,1$ & & & & & 0,2 & \\
\hline $\mathrm{INm5(LY2/gCTI)}$ & $-63,5$ & & & & & & $-5,9$ & & & & & $-2,3$ & 0,9 & \\
\hline INm6(LY2/gCT) & $-258,1$ & & & & 8,9 & & $-158,2$ & & & 180,1 & & & $-0,9$ & 8071,7 \\
\hline INm7(T30/1) & $-21,7$ & & & & & & & & & & & & & \\
\hline INm8(P10/1) & $-36,6$ & & & & & & & & & & & & $-8,8$ & \\
\hline INm9(P10/2) & & & & & & & $-151,1$ & & & & & & & \\
\hline INm10(P40/1) & $-36,6$ & & & & & & & & & & & & & \\
\hline INm11(T70/2) & 38,6 & & & & & & & & & & & 21,3 & & \\
\hline INm12(PA/2) & $-27,0$ & & & & & & & & & & & & $-5,2$ & \\
\hline INm13(P30/1) & $-199,5$ & & & & & & & & & & & & & \\
\hline INm14(P40/2) & $-34,5$ & & 1550,7 & & & & & & & & & & $-9,5$ & \\
\hline INm15(P30/2) & $-196,8$ & & 1701,3 & & & & $-232,3$ & & & $-151,0$ & & & $-20,2$ & \\
\hline INm16(T40/2) & 55,1 & & 2139,3 & & & & & 289,8 & & & & & $-15,9$ & \\
\hline INm17(T40/1) & $-2718,9$ & 8011,0 & & & & & & & & 3535,0 & 5444,6 & 98,7 & & \\
\hline INm18(TA/2) & & $-6589,4$ & & & 142,5 & 23988,5 & 1125,9 & $-1268,7$ & & & $-1742,0$ & & & \\
\hline K1(LY2/LG) & & 141759,0 & & & 2764,2 & $-39656,2$ & 14091,7 & & & & & & & \\
\hline K2(LY2/G) & & & & & & & & & & & $-1250,9$ & & & 11577,4 \\
\hline K3(LY2/AA) & $-36,2$ & $-696,0$ & 1324,4 & & & & & & & 154,4 & $-1305,2$ & & & \\
\hline K4(LY2/GH) & & & 2383,3 & & & & & & & & 1963,0 & 34,5 & & \\
\hline K5(LY2/gCTl) & $-180,8$ & & $-1036,4$ & & $-48,7$ & & & & & 232,1 & 1097,0 & & $-42,2$ & \\
\hline K6(LY2/gCT) & $-2205,7$ & 10300,1 & 9922,7 & & 123,2 & & $-1827,9$ & & & & & 310,7 & $-7,1$ & \\
\hline K7(T30/1) & & & $-6947,7$ & & & & & & & $-1973,9$ & & & $-288,5$ & \\
\hline K8(P10/1) & $-3080,4$ & & $-7633,6$ & & & & & $-699,1$ & & $-75,0$ & & & $-260,7$ & \\
\hline K9(P10/2) & & $-14813,3$ & $-15146,7$ & & & & & & 14761,6 & $-2539,0$ & $-8919,4$ & $-493,8$ & & \\
\hline K10(P40/1) & $-4381,4$ & & & & & & $-1670,7$ & $-2125,1$ & & 124,5 & $-567,1$ & 209,1 & & $-7892,9$ \\
\hline K11(T70/2) & 2323,7 & & & & $-231,2$ & & & 1879,5 & & $-1089,9$ & & & 139,0 & \\
\hline $\mathrm{K} 12(\mathrm{PA} / 2)$ & & $-12780,2$ & 9229,8 & & $-68,3$ & & & 1444,8 & & & & & & \\
\hline K13(P30/1) & 4010,6 & & & & & & $-2469,8$ & $-1320,3$ & 7745,2 & & & $-206,2$ & & \\
\hline K14(P40/2) & 4204,5 & 38221,1 & 18198,3 & & 150,4 & & & 4315,6 & & & & & & 68299,8 \\
\hline $\mathrm{K} 15(\mathrm{P30} / \mathbf{2})$ & & & $-4885,9$ & 2817,4 & & 37590,1 & & & & & 2284,4 & 256,7 & $-279,3$ & \\
\hline K16(T40/2) & 4094,7 & & 7185,5 & & & & $-2419,8$ & & & & & & 690,2 & 79394,7 \\
\hline K17(T40/1) & 24678,1 & & & & & & 16338,7 & & & $-4310,9$ & & & $-106,6$ & $-135214,0$ \\
\hline K18(TA/2) & 13607,3 & 13667,7 & & 6774,2 & & 89618,5 & 1157,8 & & 19029,0 & & & 790,0 & 900,9 & 70159,3 \\
\hline $\mathrm{R}^{2} \mathrm{Cal}^{\mathrm{b}}$ & 0,980 & 0,974 & 0,953 & 0,926 & 0,899 & 0,886 & 0,881 & 0,875 & 0,875 & 0,872 & 0,856 & 0,855 & 0,844 & 0,796 \\
\hline $\mathbf{R}^{2} \mathbf{C V}^{\mathrm{c}}$ & 0,937 & 0,827 & 0,769 & 0,864 & 0,721 & 0,787 & 0,625 & 0,735 & 0,852 & 0,786 & 0,724 & 0,412 & 0,665 & 0,646 \\
\hline RMSECV $^{\mathrm{d}}$ & 16,79 & 24,49 & 28,17 & 9,62 & 0,37 & 264,00 & 8,94 & 7,01 & 40,40 & 14,32 & 11,28 & 0,80 & 0,74 & 79,80 \\
\hline $\begin{array}{l}\text { RMSECV/Máx. } \\
(\%)^{\mathrm{e}}\end{array}$ & 13 & 17 & 16 & 11 & 10 & 14 & 16 & 15 & 14 & 12 & 20 & 22 & 14 & 21 \\
\hline
\end{tabular}

${ }^{a}$ Ecuaciones de predicción: $Y_{\text {compuesto }}=\mathrm{B}_{0}+\mathrm{a}_{1} \mathrm{IN}_{\operatorname{máx}} 1+\mathrm{a}_{2} \mathrm{IN}_{\operatorname{máx}} 2+\ldots+\mathrm{a}_{18} \mathrm{IN}_{\operatorname{máx}} 18+\mathrm{b}_{1} \mathrm{~K} 1+\mathrm{b}_{2} \mathrm{~K} 2+\ldots+\mathrm{b}_{18} \mathrm{~K}_{18}$, donde $\mathrm{IN}_{\max }$ es la intensidad máxima relativa, Ki es la pendiente para cada sensor, a $\mathrm{a}_{1} \mathrm{a}_{18}$ son los coeficientes de la ecuación de mejor ajuste.

${ }^{\mathrm{b}} \mathrm{R}^{2}$ _Cal: coeficientes de regresión de los modelos de calibración.

${ }^{c} \mathrm{R}^{2}$ CV : coeficientes de regresión de ajuste de los modelos de validación.

${ }^{d}$ RMSECV: Raíz cuadrada de la media del cuadrado del error de la validación cruzada (ng g ${ }^{-1}$ ).

e RMSECV/Máx (\%): porcentaje del cociente entre RMSECV y el valor de concentración máxima para cada compuesto. 
Tabla 4. Modelos de predicción ${ }^{\mathrm{a}}$ aproximada de contenidos de volátiles (ng $\mathrm{g}^{-1}$ ) a partir de respuestas de los sensores de nariz electrónica.

\begin{tabular}{|c|c|c|c|c|c|c|c|c|c|c|c|c|c|c|c|c|}
\hline & 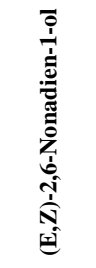 & 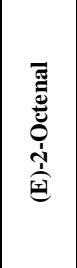 & 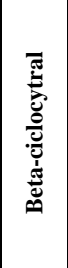 & 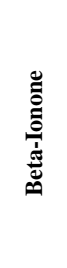 & 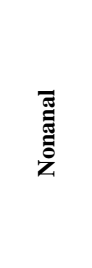 & 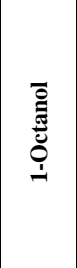 & 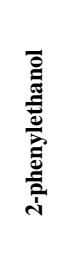 & 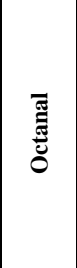 & 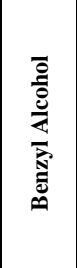 & 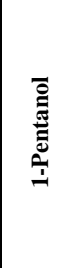 & 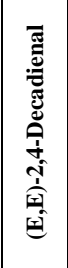 & 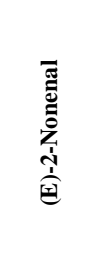 & 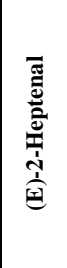 & 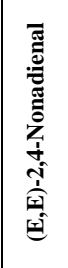 & 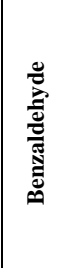 & 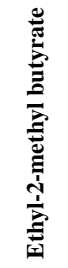 \\
\hline Bo & 2055,4 & $-105,6$ & $-6,6$ & 283,5 & 3110,8 & $-170,6$ & 9,0 & 80,9 & 69,6 & $-229,8$ & 28,7 & $-168,3$ & 1,3 & 8,6 & 20,2 & 580,1 \\
\hline INm1(LY2/LG) & & $-72,6$ & $-14,6$ & $-509,5$ & & $-59,1$ & & & $-123,9$ & $-141,5$ & $-64,1$ & & & $-32,0$ & $-38,0$ & $-677,4$ \\
\hline INm2(LY2/G) & & & 0,3 & & & & & & & & 1,4 & & & 0,3 & & \\
\hline INm3(LY2/AA) & & & 0,3 & & & & & & & & 1,1 & & & 0,3 & & \\
\hline INm4(LY2/GH) & & & 0,3 & 148,7 & & & & & & & 1,1 & & & 0,4 & & \\
\hline INm5(LY2/gCTl) & & & & 200,0 & 220,0 & & & & & & & & 3,5 & 0,5 & & \\
\hline INm6(LY2/gCT) & & & 1,2 & & & & & & & & & & 11,5 & 1,4 & & \\
\hline $\operatorname{INm7(T30/1)~}$ & & & & & & & & & & & 1,8 & & & & & \\
\hline INm8(P10/1) & & & 0,2 & $-0,3$ & & & & & & & 1,0 & & & 2,4 & & \\
\hline INm9(P10/2) & & & $-1,2$ & & & & & $-69,2$ & & & $-8,6$ & & & 3,4 & & \\
\hline INm10(P40/1) & & & 0,5 & & & & & & & & 2,8 & & & 4,6 & & \\
\hline INm11(T70/2) & & & 0,0 & $-0,8$ & & & & & & & 0,0 & & & 1,0 & & \\
\hline INm12(PA/2) & & & 0,1 & $-0,3$ & & & & & & & 0,7 & & & 1,5 & & \\
\hline INm13(P30/1) & & $-11,3$ & 0,1 & & & & & & & & 0,9 & & & 2,6 & & \\
\hline INm14(P40/2) & & $-12,6$ & $-0,5$ & $-264,3$ & & & & & & & $-1,5$ & & & 0,7 & & \\
\hline INm15(P30/2) & & $-13,2$ & $-0,3$ & $-201,7$ & & & & & & & 0,1 & & & 0,8 & & $-169,6$ \\
\hline INm16(T40/2) & 334,3 & & $-1,7$ & $-827,3$ & & & & & & & $-6,3$ & & & & & \\
\hline INm17(T40/1) & & 297,1 & 23,9 & & & 271,4 & $-1,2$ & & & 536,9 & & & 13,7 & & & \\
\hline INm18(TA/2) & $-3032,7$ & & & & $-2630,4$ & & & & & & & & & & & \\
\hline K1(LY2/LG) & $-17997,3$ & & & & & & $-560,4$ & & & & & & & & & \\
\hline K2(LY2/G) & & & & & & & & & & & 5,4 & & & 0,4 & & \\
\hline K3(LY2/AA) & & & & & & & & & & & 3,0 & & & 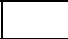 & & \\
\hline K4(LY2/GH) & & 30,7 & 3,0 & 154,6 & & & & & & & 11,4 & & & 9,4 & & \\
\hline K5(LY2/gCTl) & $-961,8$ & & 2,0 & 879,6 & & & & & & & 0,2 & & 18,3 & 2,8 & & \\
\hline K6(LY2/gCT) & & & & & & & & 119,0 & & & & & 57,4 & & & \\
\hline K7(T30/1) & & & & & $-4237,0$ & & $-55,0$ & $-164,6$ & & & & & & & & \\
\hline K8(P10/1) & & & & & & & & & & & & & & 22,3 & & \\
\hline \multicolumn{17}{|l|}{ K9(P10/2) } \\
\hline K10(P40/1) & $-3850,1$ & $-40,8$ & & & & & & & & & 77,4 & $-29051,0$ & & & & \\
\hline K11(T70/2) & 5523,8 & $-50,0$ & & & & & & & & & 0,0 & & & $-9,3$ & & \\
\hline K12(PA/2) & & & & & & & & & & & 40,7 & 6682,6 & & & & \\
\hline K13(P30/1) & & & 11,3 & 382,3 & & 318,1 & & $-154,5$ & & & & & & & & \\
\hline K14(P40/2) & & & 8,0 & 488,9 & & & & & & & $-20,8$ & $-22196,6$ & & $-59,1$ & & \\
\hline K15(P30/2) & $-6924,0$ & & & & $-4529,2$ & & & $-175,3$ & & & $-9,5$ & & & & & $-183,5$ \\
\hline K16(T40/2) & 11207,1 & $-105,1$ & & & & & & & & & 19,2 & & & $-50,8$ & & \\
\hline K17(T40/1) & & & & & $-21858,2$ & & & & & & & & & & & \\
\hline K18(TA/2) & & 112,0 & 32,6 & 114,9 & & 784,3 & & & & & 103,7 & 79416,6 & & & & 119,1 \\
\hline $\mathrm{R}^{2} \mathrm{Cal}^{\mathrm{b}}$ & 0,725 & 0,702 & 0,642 & 0,527 & 0,510 & 0,496 & 0,448 & 0,444 & 0,402 & 0,382 & 0,375 & 0,360 & 0,294 & 0,271 & 0,254 & 0,225 \\
\hline $\mathbf{R}^{2} \mathbf{C V}^{\mathrm{c}}$ & 0,422 & 0,466 & $\mathbf{0 , 3 3 0}$ & $\mathbf{0 , 3 3 5}$ & 0,379 & $\mathbf{0 , 3 7 0}$ & 0,247 & $\mathbf{0 , 3 4 9}$ & 0,246 & 0,161 & 0,111 & 0,119 & 0,065 & $0,0,019$ & 0,141 & 0,010 \\
\hline RMSECV $^{\mathrm{d}}$ & 10,18 & 1,79 & 0,32 & 1,18 & 84,23 & 4,16 & 0,44 & 3,81 & 2,98 & 5,20 & 2,05 & 170,56 & 2,19 & 1,25 & 1,21 & 3,24 \\
\hline $\begin{array}{l}\text { RMSECV/Máx. } \\
(\%)^{\mathrm{e}}\end{array}$ & 28 & 25 & 27 & 29 & 30 & 21 & 32 & 41 & 18 & 16 & 48 & 30 & 37 & 42 & 35 & 43 \\
\hline
\end{tabular}

${ }^{\mathrm{a}}$ Ecuaciones de predicción: $\mathrm{Y}_{\text {compuesto }}=\mathrm{B}_{0}+\mathrm{a}_{1} \mathrm{IN}_{\operatorname{máx}} 1+\mathrm{a}_{2} \mathrm{IN}_{\operatorname{máx}} 2+\ldots+\mathrm{a}_{18} \mathrm{IN} \mathrm{N}_{\operatorname{máx}} 18+\mathrm{b}_{1} \mathrm{~K} 1+\mathrm{b}_{2} \mathrm{~K} 2+\ldots+\mathrm{b}_{18} \mathrm{~K}_{18}$, donde IN $\mathrm{N}_{\max }$ es la intensidad máxima relativa, Ki es la pendiente para cada sensor, a1-a18 son los coeficientes de la ecuación de mejor ajuste.

${ }^{\mathrm{b}} \mathrm{R}^{2}$ _Cal: coeficientes de regresión de los modelos de calibración.

${ }^{\mathrm{c}} \mathrm{R}^{2}$ CV : coeficientes de regresión de ajuste de los modelos de validación.

d RMSECV: Raíz cuadrada de la media del cuadrado del error de la validación cruzada (ng g ${ }^{-1}$ ).

e RMSECV/Máx. (\%): porcentaje del cociente entre RMSECV y el valor de concentración máxima para cada compuesto. 


\section{AGRADECIMIENTOS}

Este estudio se ha financiado parcialmente a través del proyecto AGL2014-53398-C2-2-R concedido por el Ministerio de Economía y Competitividad en colaboración con FEDER. Alejandro Fredes agradece la financiación recibida inicialmente de la Generalitat Valenciana a través de una beca del programa "Santiago Grisolía/2013/032" y posteriormente del Banco Santander a través del programa "Ayudas para estudios de doctorado a estudiantes procedentes de America Latina" y que le han permitido su estancia de estudios en España para el desarrollo de los trabajos recogidos en este artículo.

\section{BIBLIOGRAFÍA}

Abbaszadeh, R., Moosavian, A., Rajabipour, A., Najafi, G., 2015. An intelligent procedure for watermelon ripeness detection based on vibration signals. J. Food Sci. Technol. $52,1075-1081$.

Athamneh, A., Zoecklein, B., Mallikarjunan, K., 2008. Electronic nose evaluation of Cabernet Sauvignon fruit maturity. J. Wine Res. 19, 69-80.

Beaulieu, J.C., 2006. Effect of cutting and storage on acetate and nonacetate esters in convenient, ready-to-eat fresh-cut melons and apples. HortScience 41, 65-73.

Beaulieu, J.C., Lea, J.M., 2006. Characterization and semiquantitative analysis of volatiles in seedless watermelon varieties using solid-phase microextraction. J. Agric. Food Chem. 54, 7789-7793.

Benady, M., Simon, J.E., Charles, D.J., Miles, G.E., 1995. Fruit ripeness determination by electronic sensing of aromatic volatiles. Trans. ASAE 38, 251-257.

Benedetti, S., Buratti, S., Spinardi, A., Mannino, S., Mignani, I., 2008. Electronic nose as a non-destructive tool to characterise peach cultivars and to monitor their ripening stage during shelf-life. Postharvest Biol. Technol. 47, 181-188.

Bicchi, C., Cordero, C., Liberto, E., Sgorbini, B., Rubiolo, P., 2008. Headspace sampling of the volatile fraction of vegetable matrices. J. Chromatogr. A 1184, 220-233.

Biniecka, M., Caroli, S., 2011. Analytical methods for the quantification of volatile 
aromatic compounds. TrAC - Trends Anal. Chem. 30, 1756-1770.

Brereton, R.G., Lloyd, G.R., 2014. Partial least squares discriminant analysis: taking the magic away. J. Chemom. 28, 213-225.

Brezmes, J., Llobet, E., 2016. Electronic noses for monitoring the quality of fruit, in: Rodríguez Méndez, M.L. (Ed.), Electronic noses and tongues in food science. Elsevier, London, UK, pp. 49-58.

Brezmes, J., Llobet, E., Vilanova, X., Saiz, G., Correig, X., 2000. Fruit ripeness monitoring using an electronic nose. Sensors Actuators B Chem. 69, 223-229.

Causse, M., Saliba Colombani, V., Lesschaeve, I., Buret, M., 2001. Genetic analysis of organoleptic quality in fresh market tomato. 2. Mapping QTLs for sensory attributes. Theor. Appl. Genet. 102, 273-283.

Davis, A.R., Perkins-Veazie, P., Sakata, Y., López-Galarza, S., Maroto, J.V., Lee, S.G., Huh, Y.C., Sun, Z., Miguel, A., King, S.R., Cohen, R., Lee, J.M., 2008. Cucurbit grafting. CRC. Crit. Rev. Plant Sci. 27, 50-74.

Di Natale, C., Macagnano, A., Martinelli, E., Paolesse, R., Proietti, E., D’Amico, A., 2001. The evaluation of quality of post-harvest oranges and apples by means of an electronic nose. Sensors Actuators, B Chem. 78, 26-31.

Douillard, C., Guichard, E., 1990. Characterisation of some cultivars and influence of freezing. J. Sci. food Agric. 50, 517-531.

Du, X., Bai, J., Plotto, A., Baldwin, E., Whitaker, V., Rouseff, R., 2010. Electronic nose for detecting strawberry fruit maturity, in: Proceedings of the Florida State Horticultural Society 123, pp. 259-263.

Fredes, A., Roselló, S., Beltrán, J., Cebolla-Cornejo, J., Pérez-de-Castro, A., Gisbert, C., Picó, M.B., 2017. Fruit quality assessment of watermelons grafted onto citron melon rootstock. J. Sci. Food Agric. 97, 1646-1655.

Fredes, A., Sales, C., Barreda, M., Valcárcel, M., Roselló, S., Beltrán, J., 2016. Quantification of prominent volatile compounds responsible for muskmelon and watermelon aroma by purge-and-trap extraction followed by gas chromatographymass spectrometry determination. Food Chem. 190, 689-700.

García-González, D.L., Aparicio, R., 2002. Sensors: from biosensors to the electronic nose. Grasas y Aceites 53, 96-114. 
Gardner, J.W., Bartlett, P.N., 1999. Electronic noses. Principles and applications. Meas. Sci. Technol. 11, 1087.

Gardner, J.W., Persaud, K.C., 2001. Electronic noses and olfaction 2000. Meas. Sci. Technol. 12, 2019-2020.

Guarrasi, V., Farina, V., Germanà, M.A., San Biagio, P.L., Mazzaglia, A., 2011. Fruit quality evaluation of four loquat cultivars grown in Sicily. Acta Hortic. 887, 299304.

Hernández, A., Wang, J., Hu, G., García Pereira, A., 2008. Monitoring storage shelf life of tomato using electronic nose technique. J. Food Eng. 85, 625-631.

Holmberg, M., Artursson, T., 2003. Drift compensation, standards, and calibration methods, in: Pearce, T.C., Schiffman, S.S., Nagle, H.T., Gardner, J.W. (Eds.), Handbook of machine olfaction. Wiley-VCH Verlag GmbH \& Co. KGaA, Weinheim, Germany, pp. 325-346.

Hubert, M., Vanden Branden, K., 2003. Robust methods for partial least squares regression. J. Chemom. 17, 537-549.

Jackson, J.E., Mudholkar, G.S., 1979. Control procedures for residuals associted with principal component analysis. Technometrics $21,341-349$.

King, S.R., Davis, A.R., Zhang, X., Crosby, K., 2010. Genetics, breeding and selection of rootstocks for Solanaceae and Cucurbitaceae. Sci. Hortic. 127, 106-111.

Kovats, E., 1958. Gas-chromatographische charakterisierung organischer verbindungen. Teil 1: retentionsindices aliphatischer halogenide, alkohole, aldehyde und ketone. Helv. Chim. Acta 41, 1915-1932.

Krzanowski, W.J., 2000. Principles of multivariate analysis : a user's perspective. Oxford University Press Inc., New York, USA.

Lebrun, M., Ducamp-Collin, M.-N., Plotto, A., Goodner, K.L., Baldwin, E., 2004. Development of electronic nose measurements for mango (Mangifera indica) homogenate and whole fruit, in: Proceedings of the Annual Meeting of the Florida State Horticultural Society. pp. 421-425.

Lewinsohn, E., Sitrit, Y., Bar, E., Azulay, Y., Meir, A., Zamir, D., Tadmor, Y., 2005. Carotenoid pigmentation affects the volatile composition of tomato and watermelon fruits, as revealed by comparative genetic analyses. J. Agric. Food Chem. 53, 3142- 
3148.

Lin, S., Zhang, X., 2016. A rapid and novel method for predicting nicotine alkaloids in tobacco through electronic nose and partial least-squares regression analysis. Anal. Methods 8, 1609-1617.

Liu, B., Ren, J., Zhang, Y., An, J., Chen, M., Chen, H., Xu, C., Ren, H., 2015. A new grafted rootstock against root-knot nematode for cucumber, melon, and watermelon. Agron. Sustain. Dev. 35, 251-259.

Llobet, E., Hines, E.L., Gardner, J.W., Franco, S., 1999. Non-destructive banana ripeness determination using a neural network-based electronic nose. Meas. Sci. Technol. $10,538-548$.

Marrazzo, W.N., Heinemann, P.H., Crassweller, R.E., Leblanc, E., 2005. Electronic nose chemical sensor feasibility study for the differentiation of apple cultivars. 48, 19952002.

Pino, J.A., Marbot, R., Aguero, J., 2003. Volatile components of watermelon (Citrullus lanatus [Thunb.] Matsum. et Nakai) fruit. J. Essent. Oil Res. 15, 379-380.

Rouphael, Y., Schwarz, D., Krumbein, A., Colla, G., 2010. Impact of grafting on product quality of fruit vegetables. Sci. Hortic. 127, 172-179.

Simon, J.E., Hetzroni, A., Bordelon, B., Miles, G.E., Charles, D.J., 1996. Electronic sensing of aromatic volatiles for quality sorting of blueberries. J. Food Sci. 61, 967-970.

Sinesio, F., Di Natale, C., Quaglia, G.B., Bucarelli, F.M., Moneta, E., Macagnano, A., Paolesse, R., D'Amico, A., 2000. Use of electronic nose and trained sensory panel in the evaluation of tomato quality. J. Sci. Food Agric. 80, 63-71.

Solis-Solis, H.M.M., Calderon-Santoyo, M., Gutierrez-Martinez, P., Schorr-Galindo, S., Ragazzo-Sanchez, J.A.A., 2007. Discrimination of eight varieties of apricot (Prunus armeniaca) by electronic nose, LLE and SPME using GC-MS and multivariate analysis. Sensors Actuators B 125, 415-421.

Song, S., Zhang, X., Hayat, K., Jia, C., Xia, S., Zhong, F., Xiao, Z., Tian, H., Niu, Y., 2010. Correlating chemical parameters of controlled oxidation tallow to gas chromatography-mass spectrometry profiles and e-nose responses using partial least squares regression analysis. Sensors Actuators, B Chem. 147, 660-668. 
Thies, J.A., Ariss, J.J., Hassel, R.L., Olson, S., Kousik, C.S., Levi, A., 2010. Grafting for management of southern root-knot nematode, Meloidogyne incognita, in watermelon. Plant Dis. 94, 1195-1199.

Tieman, D., Zhu, G., Resende, M.F.R., Lin, T., Nguyen, C., Bies, D., Rambla, J.L., Ortiz, K.S., Taylor, M., Zhang, B., Ikeda, H., Liu, Z., Fisher, J., Zemach, I., Monforte, A., Zamir, D., Granell, A., Kirst, M., Huang, S., Klee, H., 2017. A chemical genetic roadmap to improved tomato flavor. Plant Sci. 355, 391-394.

Valcárcel, M., 2009. Optimización del proceso de evaluación y seleeción de germoplasma de tomate por características de calidad organoléptica: uso de la tecnología NIR y sensores electrónicos. PhD. Thesis. Escola Superior de Tecnologia i Ciències Experimentals - Departament de Ciències Agràries i del Medi Natural, Universitat Jaume I, Castellón de la Plana, Spain.

Verzera, A., Dima, G., Tripodi, G., Ziino, M., 2011. Fast quantitative determination of aroma volatile constituents in melon fruits by headspace-solid-phase microextraction and gas chromatography-mass spectrometry. Food Anal. Methods $4,141-149$.

Vinson, E.L., Woods, F.M., Kemble, J.M., Perkins-Veazie, P., Davis, A., Kessler, J.R., 2010. Use of external indicators to predict maturity of mini-watermelon fruit. HortScience 45, 1034-1037.

Wise, B.M., Gallagher, N.B., Bro, R., Shaver, J.M., Windig, W., Koch, R.S., 2006. Chemometrics Tutorial for PLS_Toolbox and Solo, MATLABTM, 4th ed, Eigenvector Research, Inc.

Xisto, A.L.R.P., Vilas Boas, E.V. de B., Nunes, E.E., Vilas Boas, B.M., Guerreiro, M.C., 2012. Volatile profile and physical, chemical, and biochemical changes in fresh cut watermelon during storage. Ciência e Tecnol. Aliment. 32, 173-178.

Yajima, I., Sakakibara, H., Ide, J., Yanai, T., Hayashi, K., 1985. Volatile flavor components of watermelon (Citrullus vulgaris). Agric. Biol. Chem. 49, 3145-3150.

Yang, S.L., Xie, S.P., Xu, M., Zhang, C., Wu, N., Yang, J., Zhang, L., Zhang, D.Y., Jiang, Y., Wu, C.J., 2015. A novel method for rapid discrimination of bulbus of Fritillaria by using electronic nose and electronic tongue technology. Anal. Methods 7, 943952. 
Yativ, M., Harary, I., Wolf, S., 2010. Sucrose accumulation in watermelon fruits: genetic variation and biochemical analysis. J. Plant Physiol. 167, 589-596.

Yoo, K.S., Bang, H., Lee, E.J., Crosby, K., Patil, B.S., 2012. Variation of carotenoid, sugar, and ascorbic acid concentrations in watermelon genotypes and genetic analysis. Hortic. Environ. Biotechnol. 53, 552-560.

Young, H., Rossiter, K., Wang, M., Miller, M., 1999. Characterization of Royal Gala apple aroma using electronic nose technology-potential maturity indicator. J. Agric. Food Chem. 47, 5173-5177.

Zawirska-Wojtasiak, R., Gośliński, M., Szwacka, M., Gajc-Wolska, J., Mildner-Szkudlarz, S., 2009. Aroma evaluation of transgenic, thaumatin II-producing cucumber fruits. J. Food Sci. 74, 204-210.

Zhang, H., Wang, J., Ye, S., 2008. Predictions of acidity, soluble solids and firmness of pear using electronic nose technique. J. Food Eng. 86, 370-378. 


\section{CONCLUSIONES GENERALES}



Abordar la presente tesis ha permitido desarrollar herramientas para el fenotipado de la calidad organoléptica de melón y sandía y su aplicación a la evaluación de líneas de mejora de portainjertos de sandía. En particular, de los resultados obtenidos se extraen las siguientes conclusiones:

- Se ha desarrollado un método de análisis de compuestos volátiles, optimizado para melón y sandía. Este método es sencillo y representa costes relativamente reducidos, permitiendo la cuantificación de los compuestos con mayor exactitud que otras técnicas basadas en métodos complejos y tediosos (adicciones estándar o calibración de matriz). Entre las ventajas del método también destaca la posibilidad de extraer varias muestras al mismo tiempo, ahorro de tiempo de análisis, posibilidad de almacenar parte del extracto de la muestra para poder realizar análisis retrospectivos para la identificación de compuestos desconocidos o su comparación en otros equipos más potentes.

- Respecto a la extracción purga y trampa donde se utilizaron dos fases adsorbentes (TENAX y ENVI-Carb) concluimos que ENVI-Carb presentó resultados comparables con TENAX, pero a mitad de coste. El análisis mediante superficies de respuesta multivariante permitió fijar las condiciones óptimas de extracción: masa de muestra 30 g, tiempo de extracción 49 min y flujo de gas portador 1.6 L.min ${ }^{-1}$.

- Con el método desarrollado se logró comprobar la presencia de los principales compuestos del aroma de melón y sandía logrando cuantificar hasta 47 volátiles en las muestras de melón climatérico del tipo Charentais. Los datos obtenidos están de acuerdo con la literatura actual. Las muestras de sandía presentaron mayor acumulación de los compuestos Geranylacetona, (Z)-3-nonen-1-ol, $\beta$-ionona, (E)-2heptenal y (E,Z)-2,6-nonadien-1-ol en comparación con las muestras de melón. Además el perfil de volátiles del melón tipo Charentais resultó el más complejo ya que presentó una mayor diversidad y concentración de compuestos en comparación con los demás. 
- El método desarrollado se ha aplicado a la evaluación de líneas de mejora de portainjertos de sandía, identificando los efectos del patrón sobre la calidad organoléptica del fruto.

- El efecto de las líneas evaluadas sobre el perfil de azúcares y ácidos de la sandía es limitado. En el caso de la línea más interesante, de citroides, aumentan ligeramente los contenidos de glucosa y ácido málico.

- La línea empleada como portainjertos sí que puede tener un efecto mayor sobre el perfil volátil de la sandía. La línea de citroides evaluada, presenta el perfil más similar al control sin injertar o autoinjertado. En particular, no presenta los niveles aumentados de Z-6-nonen-1-ol de los materiales más habituales de C. maxima x C. moschata que proporciona notas a calabaza.

- El bajo impacto del portainjerto melón citroides sobre la calidad de los frutos, así como la resistencia contra nematodos, hacen de este portainjerto una alternativa prometedora para los cultivos de Cucurbita.

- Se ha optimizado un protocolo de análisis del perfil volátil de sandía mediante nariz electrónica y se ha aplicado a la evaluación de líneas de mejora de portainjertos de sandía

- La utilización de métodos de clasificación empleando las huellas electrónicas de la nariz no permite generalizar la clasificación de frutos de la misma combinación portainjerto-variedad. Ello puede deberse bien a la similitud de los materiales, bien a efectos micro-ambientales importantes. En este caso, efectos micro-ambientales que pueden estar relacionados con diferencias climáticas menores en la parcela de cultivo, efectos del desarrollo del injerto o diferencias en el estado de maduración debido a la dificultad del muestreo en esta especie, hace que determinadas muestras presenten un perfil volátil particular. 
- En cualquier caso, un análisis tipo PLS-DA o PCA permite determinar claramente las similitudes o diferencias en el perfil volátil de las muestras evaluadas, mostrando una clara coherencia con los mismos perfiles obtenidos por cromatografía de gases. Así es posible obtener mapas de perfiles volátiles mediante nariz electrónica que permiten seleccionar los materiales que manifiesten una similitud en la acumulación de compuestos volátiles o que se acerquen a un objetivo ideal.

- A pesar de tratarse de un método de análisis sin separación, el uso de modelos iPLS con las huellas electrónicas del perfil de volátiles permite seleccionar materiales que presenten mayor o menor acumulación de compuestos concretos. En este caso tiene interés por poder identificar las muestras con menor acumulación de Z-6-nonen-1-ol.

- La utilización de nariz electrónica en la evaluación del perfil volátil de sandía, permitirá prescindir de métodos más caros y sobre todo más lentos de análisis como la cromatografía de gases, en la evaluación preliminar de grandes cantidades de materiales. Una situación habitual en los programas de mejora. De esta forma se podrán aplicar las herramientas más precisas a un menor número de materiales preseleccionados. 

ANEXO 

Ana María Pérez de Castro, Profesora Contratada Doctora de la Universitat Politècnica de València,

\section{HACE CONSTAR QUE:}

Como coautora de las publicaciones indicadas más abajo, acepto que ésta se presente como parte de la Tesis Doctoral de Alejandro David Fredes Sivoplás y renuncio expresamente a su utilización como parte de otra tesis doctoral. Además, certifico que esta publicación no se han incluido previamente en ninguna otra tesis doctoral.

Fruit quality assessment of watermelons grafted onto citron melon rootstock. A. Fredes, $S$. Roselló, J. Beltrán, J. Cebolla-Cornejo, A. Pérez-de-Castro, C. Gisbert, M.B. Picó. 2016. Journal of the Science of Food and Agriculture, 97(5): 1646-1655.

Y para que conste a los efectos oportunos, así lo firmo.

En Valéncia, a 3 de julio de 2017

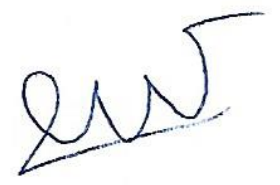

Fdo.: Ana María Pérez de Castro 
Maria Belén Picó Sirvent, Catedrática de Universidad de la Universitat Politècnica de València,

\section{HACE CONSTAR QUE:}

Como coautora de las publicaciones indicadas más abajo, acepto que éstas se presenten como parte de la Tesis Doctoral de Alejandro David Fredes Sivoplás y renuncio expresamente a su utilización como parte de otra tesis doctoral. Además, certifico que estas publicaciones no se han incluido previamente en ninguna otra tesis doctoral.

Fruit quality assessment of watermelons grafted onto citron melon rootstock. A. Fredes, S. Roselló, J. Beltrán, J. Cebolla-Cornejo, A. Pérez-de-Castro, C. Gisbert, M.B. Picó. 2016. Journal of the Science of Food and Agriculture, 97(5): 1646-1655.

Use of electronic nose as a phenotyping tool in watermelon: application to the evaluation of rootstock lines. A. Fredes, J. Cebolla-Cornejo, J. Beltrán, C. Gisbert, M.B. Picó, M. Valcárcel, S. Roselló (pendiente envio a Food Chemistry).

Y para que conste a los efectos oportunos, así lo firmo.

En Valéncia, a 3 de julio de 2017

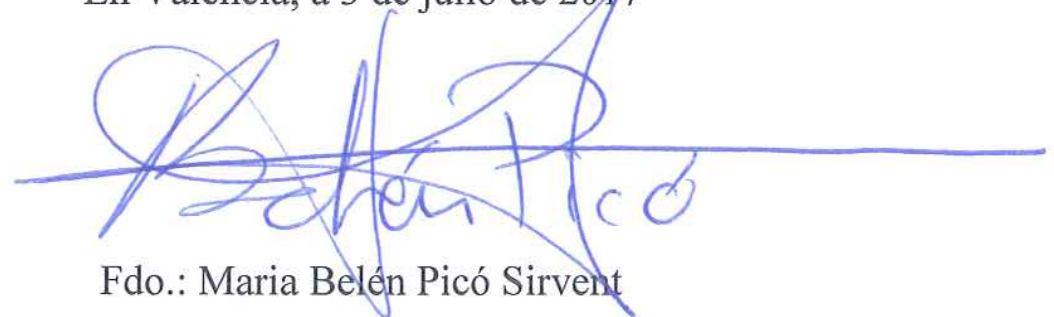


Carlos Sales Martínez, Personal Investigador de la Universitat Jaume I de Castellón,

\section{HACE CONSTAR QUE:}

Como coautor de la publicación indicada más abajo, acepto que ésta se presente como parte de la Tesis Doctoral de Alejandro David Fredes Sivoplás y renuncio expresamente a su utilización como parte de otra tesis doctoral. Además, certifico que esta publicación no se ha incluido previamente en ninguna otra tesis doctoral.

Quantification of prominent volatile compounds responsible for muskmelon and watermelon aroma by purge and trap extraction followed by gas chromatography-mass spectrometry determination. A. Fredes, C. Sales, M. Barreda, M. Valcárcel, S. Roselló, J. Beltrán. 2016. Food Chemistry 190: 689-700.

Y para que conste a los efectos oportunos, así lo firmo.

En Castellón de la Plana, a 3 de julio de 2017

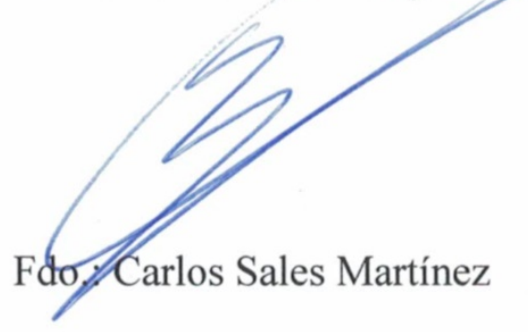


Jaime Cebolla Cornejo, Profesor Contratado Doctor de la Universitat Politècnica de

València,

\section{HACE CONSTAR QUE:}

Como coautor de las publicaciones indicadas más abajo, acepto que éstas se presenten como parte de la Tesis Doctoral de Alejandro David Fredes Sivoplás y renuncio expresamente a su utilización como parte de otra tesis doctoral. Además, hago constar que estas publicaciones no se han incluido previamente en ninguna otra tesis doctoral.

Fruit quality assessment of watermelons grafted onto citron melon rootstock. A. Fredes, S. Rosello, J. Beltrán, J. Cebolla-Cornejo, A. Pérez-de-Castro, C. Gisbert, M.B. Picó. 2016. Journal of the Science of Food and Agriculture, 97(5): 1646-1655.

Use of electronic nose as a phenotyping tool in watermelon: application to the evaluation of rootstock lines. A. Fredes, J. Cebolla-Cornejo, J. Beltrán, C. Gisbert, M.B. Picó, M. Valcárcel, S. Roselló (pendiente envio a Food Chemistry).

Y para que conste a los efectos oportunos, así lo firmo.

En Valéncia, a 3 de julio de 2017

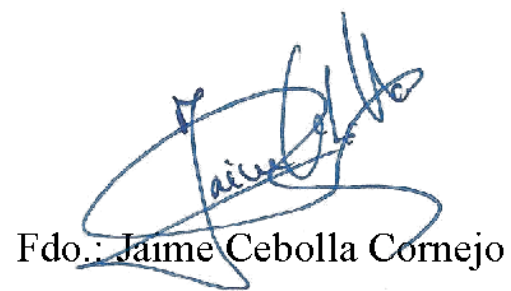


Joaquim Beltrán Arandes, Profesor Titular de Universidad de la Universitat Jaume I de

Castellón,

\section{HACE CONSTAR QUE:}

Como coautor de las publicaciones indicadas más abajo, acepto que éstas se presenten como parte de la Tesis Doctoral de Alejandro David Fredes Sivoplás y renuncio expresamente a su utilización como parte de otra tesis doctoral. Además, certifico que estas publicaciones no se han incluido previamente en ninguna otra tesis doctoral.

Quantification of prominent volatile compounds responsible for muskmelon and watermelon aroma by purge and trap extraction followed by gas chromatography-mass spectrometry determination. A. Fredes, C. Sales, M. Barreda, M. Valcárcel, S. Roselló, J. Beltrán. 2016. Food Chemistry 190: 689-700.

Fruit quality assessment of watermelons grafted onto citron melon rootstock. A. Fredes, $S$. Roselló, J. Beltrán, J. Cebolla-Cornejo, A. Pérez-de-Castro, C. Gisbert, M.B. Picó. 2016. Journal of the Science of Food and Agriculture, 97(5): 1646-1655.

Use of electronic nose as a phenotyping tool in watermelon: application to the evaluation of rootstock lines. A. Fredes, J. Cebolla-Cornejo, J. Beltrán, C. Gisbert, M.B. Picó, M. Valcárcel, S. Roselló (pendiente envío a Food Chemistry).

Y para que conste a los efectos oportunos, así lo firmo.

En Castellón de la Plana, a 3 de julio de 2017

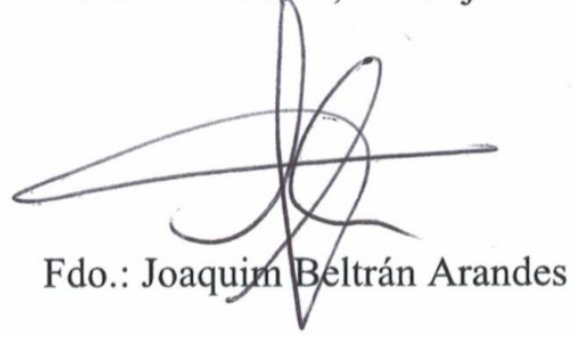


Mercedes Barreda Portalés, Técnica superior de laboratorio de la Universitat Jaume I de Castellón,

\section{HACE CONSTAR QUE:}

Como coautora de la publicación indicada más abajo, acepto que ésta se presente como parte de la Tesis Doctoral de Alejandro David Fredes Sivoplás y renuncio expresamente a su utilización como parte de otra tesis doctoral. Además, certifico que esta publicación no se ha incluido previamente en ninguna otra tesis doctoral.

Quantification of prominent volatile compounds responsible for muskmelon and watermelon aroma by purge and trap extraction followed by gas chromatography-mass spectrometry determination. A. Fredes, C. Sales, M. Barreda, M. Valcárcel, S. Roselló, J. Beltrán. 2016. Food Chemistry 190: 689-700.

Y para que conste a los efectos oportunos, así lo firmo.

En Castellón de la Plana, a 3 de julio de 2017

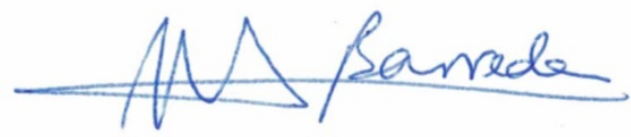

Fdo.: Mercedes Barreda Portalés 
Mercedes Valcárcel Germes, Profesora Asociada de la Universitat Jaume I de Castellón,

\section{HACE CONSTAR QUE:}

Como coautora de las publicaciones indicadas más abajo, acepto que éstas se presenten como parte de la Tesis Doctoral de Alejandro David Fredes Sivoplás y renuncio expresamente a su utilización como parte de otra tesis doctoral. Además, certifico que estas publicaciones no se han incluido previamente en ninguna otra tesis doctoral.

Quantification of prominent volatile compounds responsible for muskmelon and watermelon aroma by purge and trap extraction followed by gas chromatography-mass spectrometry determination. A. Fredes, C. Sales, M. Barreda, M. Valcárcel, S. Roselló, J. Beltrán. 2016. Food Chemistry 190: 689-700.

Use of electronic nose as a phenotyping tool in watermelon: application to the evaluation of rootstock lines. A. Fredes, J. Cebolla-Cornejo, J. Beltrán, C. Gisbert, M.B. Picó, M. Valcárcel, S. Roselló (pendiente envío a Food Chemistry).

Y para que conste a los efectos oportunos, así lo firmo.

En Castellón de la Plana, a 3 de julio de 2017

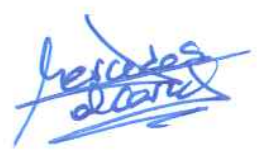

Fdo.: Mercedes Valcárcel Germes 
Salvador Antonio Roselló Ripollés, Catedrático de Universidad de la Universitat Jaume I de Castellón,

\section{HACE CONSTAR QUE:}

Como coautor de las publicaciones indicadas más abajo, acepto que éstas se presenten como parte de la Tesis Doctoral de Alejandro David Fredes Sivoplás y renuncio expresamente a su utilización como parte de otra tesis doctoral. Además, certifico que estas publicaciones no se han incluido previamente en ninguna otra tesis doctoral.

Quantification of prominent volatile compounds responsible for muskmelon and watermelon aroma by purge and trap extraction followed by gas chromatography-mass spectrometry determination. A. Fredes, C. Sales, M. Barreda, M. Valcárcel, S. Roselló, J. Beltrán. 2016. Food Chemistry 190: 689-700.

Fruit quality assessment of watermelons grafted onto citron melon rootstock. A. Fredes, S. Roselló, J. Beltrán, J. Cebolla-Cornejo, A. Pérez-de-Castro, C. Gisbert, M.B. Picó. 2016. Journal of the Science of Food and Agriculture, 97(5): 1646-1655.

Use of electronic nose as a phenotyping tool in watermelon: application to the evaluation of rootstock lines. A. Fredes, J. Cebolla-Cornejo, J. Beltrán, C. Gisbert, M.B. Picó, M. Valcárcel, S. Roselló (pendiente envio a Food Chemistry).

Y para que conste a los efectos oportunos, así lo firmo.

En Castellón de la Plana, a 3 de julio de 2017

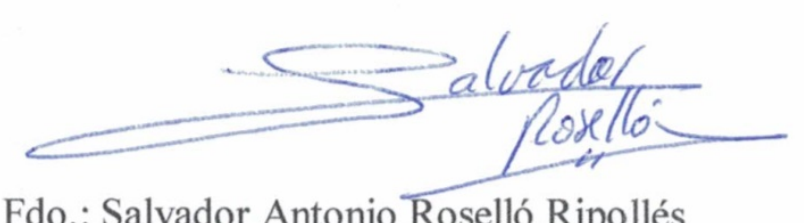

Fdo.: Salvador Antonio Roselló Ripollés 

